Preliminary Development of the Hand and Arm Function Measure for People with Neurological Conditions

By

Namrata Grampurohit

A dissertation submitted in partial fulfillment of the requirements for the degree of

Doctor of Philosophy

University of Washington

2016

Reading Committee:

Deborah Kartin, PhD, Chair

Brian Dudgeon, $\mathrm{PhD}$

Catherine Taylor, $\mathrm{PhD}$

Valerie Kelly, $\mathrm{PhD}$

Kathryn Yorkston, PhD

Program Authorized to offer degree:

Rehabilitation Science 
C) Copyright 2016

Namrata Grampurohit 
University of Washington

\begin{abstract}
Preliminary Development of the Hand and Arm Function Measure for People with Neurological Conditions
\end{abstract}

Namrata Grampurohit

Chair of the Supervisory Committee:

Deborah Kartin, PT, PhD

Department of Rehabilitation Medicine

Background: Many people with neurological conditions experience difficulty with their hands and arms. Measures for assessing hand and arm function with sound psychometric properties are needed. Understanding patient/client's perceptions of their hand use and the quality and speed of task performance, while critical, are not combined in a comprehensive measure.

Purpose: To develop Hand and Arm Function Measure (HAFM) with stakeholder engagement, that is performance-based, and includes self-report.

Method: A systematic review of literature of hand and arm function measures was conducted and psychometric properties summarized. Four experts, two occupational 
therapists, a physical therapist and a psychometrician, participated in a focus group discussing the construct, early item bank development, and later participated in item writing and modification. People with stroke $(n=7)$, traumatic brain injury $(n=2)$, multiple sclerosis $(\mathrm{n}=5)$ and Parkinson disease $(\mathrm{n}=6)$ participated in seven focus groups and eight cognitive interviews, sharing their experiences of hand and arm function difficulties in daily life and during the HAFM administration.

Results: Twenty-two measures of hand and arm function were reported in literature in the past decade. Analysis revealed, they lacked psychometric rigor, ignored the sensory capability of the hand, and were inadequately studied in people with neurological conditions. The construct definition was developed based on the frameworks of International Classification of Functioning, Disability and Health and the principles of Evidence Centered Design. The experts helped define the construct, develop the preliminary item bank, and modify items. The focus groups of people with neurological conditions helped identify items and aspects of hand function that were important to them. All items were pooled and a preliminary set of self-report and performance-based items was developed for use in the cognitive interviews. Based on the cognitive interviews, HAFM items needing modification or suggested for addition/deletion to the HAFM were nominated.

Conclusions: Data gathered from literature review, theoretical and measurement frameworks, and the stakeholders contributed to the face and content-related evidence for validity of the HAFM scores. Plans for future HAFM development include piloting the preliminary item set with people with neurological conditions and ultimately proceeding to item tryouts and large-scale administration. 


\section{Plain Language Summary}

People with neurological conditions often have problems with their hands and arms. It is important to have tests that measure changes in hand and arm use. New tests need to be developed with care to make sure they give us useful information. Tests should include tasks that people can do and a way for people to tell us about their own experiences with hand and arm use. Right now no tests address both these areas. This is why a new test, the Hand and Arm Function Measure (HAFM), is being developed.

First we read a lot about existing hand and arm function tests. We also got feedback from people with neurological conditions and experts. Four experts had a discussion about hand and arm function and came up with some questions and tasks that could be used in the test. Twenty people with neurological conditions also had group discussions about hand and arm use and eight of them were individually interviewed. They told us about their problems with their hands and arms in daily life and their experiences when they were tested with an early version of the HAFM. They suggested items to include in the HAFM.

We found twenty-two tests of hand and arm function over the past ten years. Those tests were not rigorously developed. They had not been studied in people with neurological problems. Based on theories and feedback from the experts and people with neurological problems, we came up with our own definition or construct of hand and arm function. Items from experts and people with neurological conditions were pooled and a set of items was developed for the HAFM. More input from the experts and people with neurological conditions was used to modify the HAFM items. More work needs be done 
on the HAFM including testing more people with neurological conditions and healthy adults. 
Human Subject's Application Number: 47121

Approval Date: 04/24/2014

Approved Renewal Date: 04/24/2015

Institutional Review Board: University of Washington 


\section{Acknowledgements}

It is a great pleasure to have the opportunity to thank the many wonderful people in my life whose strong support and dedication, has made this dissertation possible.

First, I would like to express my sincere and deepest gratitude to my advisor and mentor, Dr. Deborah Kartin, who has been a powerhouse of encouragement, guidance and knowledge and mentored my journey to becoming a scientist. Among the many intense phases of the project, I am indebted to Dr. Kartin for the long hours she has spent with supervising and helping with the qualitative data from collection to coding and analysis. This behemoth task would not have been complete without her.

I would like to sincerely thank my mentor, Dr. Brian Dudgeon, who guided my $\mathrm{PhD}$ journey in the beginning years, patiently listened to every new idea I presented to him, and continued to guide this dissertation research remotely. Dr. Dudgeon's expertise in occupational therapy and qualitative research provided invaluable guidance for the project.

I feel fortunate to have trained with Dr. Catherine Taylor, in instrument development and am deeply thankful for her guidance throughout my dissertation. I have greatly benefitted from her willingness to share her wisdom and expertise in measurement science and aspire to someday unravel the mysteries of the "theory of validity' to my students as tactfully as they were unraveled to me by Dr. Taylor. I sincerely appreciate her commitment to teaching and staying on my committee even with the many changes presented through the years.

I am immensely thankful to Dr. Valerie Kelly for sharing her expertise in movement science and neural control and supporting me through this dissertation. I would also like to thank Dr. Kathryn Yorkston for her guidance in organizing the systematic review and qualitative methods. I thank Dr. Nancy Woods and Dr. Basia Belza for their support. I have also been fortunate to be able to consult with Dr. Dagmar Amtmann, Dr. Janet Powell, Dr. Christine Chen, and Dr. Wendy Coster (Boston Rehabilitation Outcomes Center Consultation Services) on this project and would like to thank them for their willingness to share their knowledge with me and for the insightful discussions.

I would like to thank Walter C. and Anita C. Stolov Research Fund for funding this research project. I would like to thank Dr. Peter Esselman, Chair of the Department of Rehabilitation Medicine, University of Washington and all the staff and students that I have worked with over the years for their support, and teaching and research opportunities. I would also like to acknowledge the inspiration I have found in Dr. Jean Deitz's pioneering work in Occupational Therapy at the University of Washington and thank her for her willingness to guide me through the decision to pursue this degree. I would like to thank Dr. Sarah Westcott-McCoy, Dr. Chet Moritz and Dr. Mark Guthrie for support within the Physical Therapy Division. I would also like to thank Victoria Corrin, Laura Robinson, and Mary Garcia for their support.

I would like to acknowledge and thank from the bottom of my heart, Dr. Sujata Pradhan, who said, "You can do it" from my very first day in the PhD program. Her informal mentoring has kept me afloat through the highs and lows, which were in plenty during the last few years. On the same note, I would like to thank the many PhD students

and post-docs in the cohorts senior to me for the valuable pieces of advice. I would like to 
give a shout out to my PhD cohort, Dr. LinYa Hsu, Dr. Sara Morgan, and Dr. Torey Gilbertson for being fabulous friends.

There are many wonderful people in my life outside of the University of Washington who enabled me to complete this dissertation. Foremost, I would like to thank Renee King, for adjusting many different schedules in the hospital to make my part-time clinical work at Evergreen Health work for me through the $\mathrm{PhD}$ years. I was fortunate to add Evergreen Health as another clinical site for data collection through the support of Dr. Ted Brown, Dr. Pinky Agarwal, Dr. Marlan Kay, Dr. James Song, Kay Ballen, Ann Zylstra, Kirk Hanson, Susan Watters, Jesika Shah and Sumedha Kukreja.

I would also like to thank my participants in this research project and my patients through the years who have inspired me to search for better tests and outcomes in rehabilitation, making the research pursuits worthwhile. I would like to thank the many therapists who have shared their clinical expertise with me.

Finally, a special thank you to my loving family in US and India, who have always believed in me. I deeply thank my amazing parents, Dr. Dattatraya Grampurohit and Dr. Nirmala Grampurohit, whose own $\mathrm{PhD}$ endeavors and successful careers have predisposed me to seek the completion of this degree. I would like to greatly thank my sister, Shweta Grampurohit for her support and volunteering to do the illustrations for the Hand and Arm Function Measure designed in this research. Thanks to my brother-in-law, Dr. Ajinkya More for assisting with the illustrations and for his encouragement.

A special thank you to my enthusiastic sister-in-law Gauri Khandekar and brother-in-law Dr. Sidharth Chiravarambath for their ardent support and patience, letting me work on my dissertation writing through the Thanksgiving holidays. I am thankful for the smiles shared by Om and Meera to liven up some of the toughest days. Thanks to my parents-in-law Saktan Chiravarambath and Hemlata Saktan for celebrating my achievements.

I would like to thank my son, Neil Chiravarambath for his love, saying 'its okay' when things were not perfect, his interest in always looking at my new drafts, and keeping me excited about graduating from school. I would like to thank my daughter Keya Chiravarambath for her love, the many hugs that gave me strength and her help in organizing my dissertation papers and books.

I am eternally grateful to my beloved husband, Kapil Chiravarambath, who is the reason I was able to start and finish this degree. He has nurtured my aspirations, given me strength and helped raise a beautiful family with twenty years of love and togetherness. 
For Kapil and my family 


\section{Table of Contents}

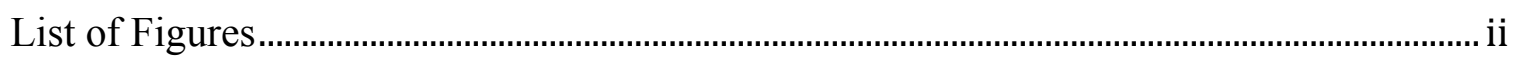

List of Tables ...........................................................................................................................ii

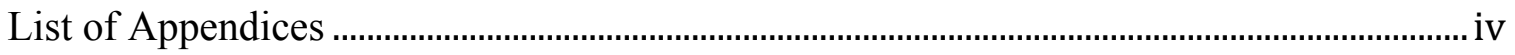

Chapter 1: Introduction.............................................................................................................. 1

Chapter 2: Literature Review …………………………………………………………..... 11

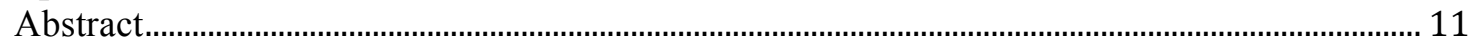

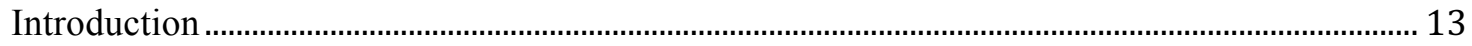

Models of classification of hand and arm function....................................................................... 13

Neural Control of Hand and Arm ................................................................................................ 15

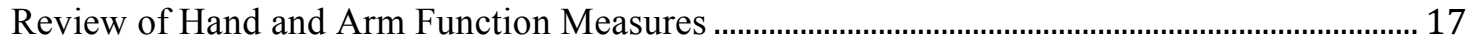

Chapter 3: Construct Definition and Expert Panel Study ........................................................ 60

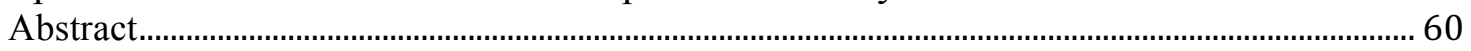

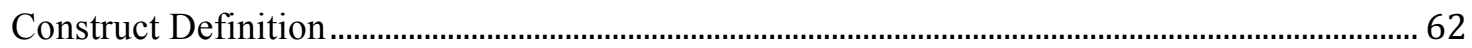

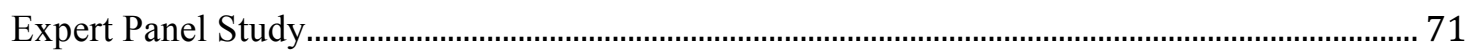

Discussion and Conclusion .............................................................................................................. 83

Chapter 4: Focus Groups and Cognitive Interviews Study ………………………………... 94

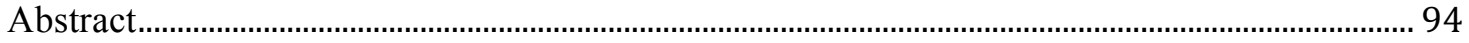

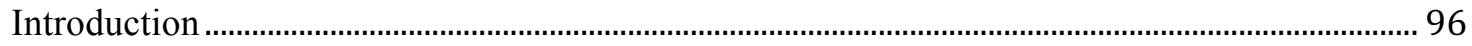

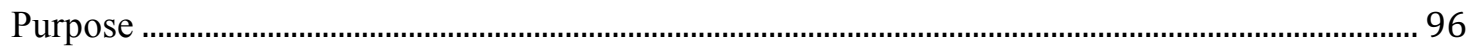

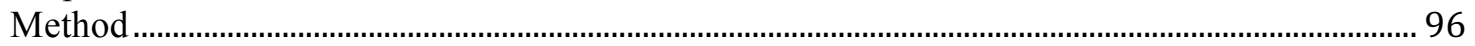

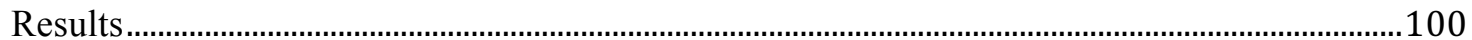

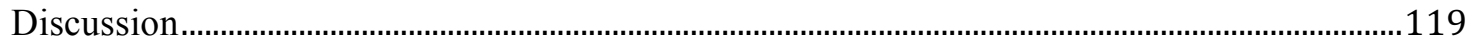

Conclusion ......................................................................................................................................123

Chapter 5: Discussion and Future Research ........................................................................132

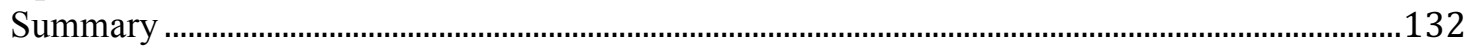

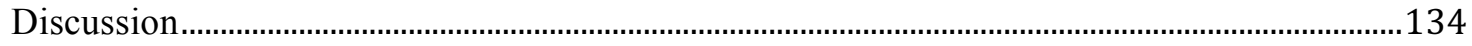

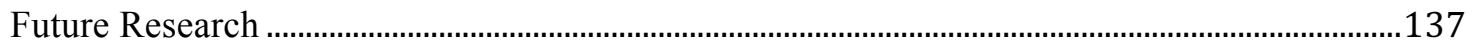

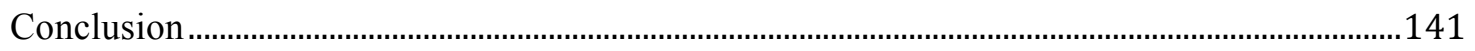

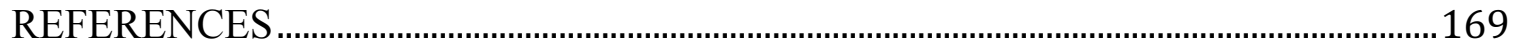




\section{List of Figures}

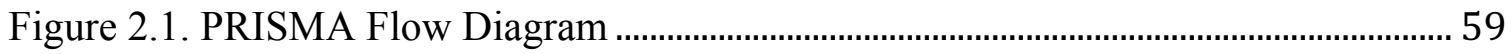

Figure 3.1. Nomological Network for Hand and Arm Function Measure............................ 90

Figure 3.2. Construct Map for Hand and Arm Function Measure ........................................ 91

Figure 3.3. Toulmin Diagram for Hand and Arm Function Measure .................................. 92

Figure 3.4. Representation of the Four Stages in Research Study ....................................... 93

Figure 4.1. Materials Used for Performance-based Section of the Measure ......................129

Figure 4.2. Manual Ability Measure Scores by Diagnosis .................................................130

Figure 4.3. Item Development Process: Schematic Flow Diagram from Item Bank

Development to Preliminary Selection of Items...............................................................131 


\section{List of Tables}

Table 1.1. Descriptions of Arm and Hand Issues in Neurological Conditions ................... 10

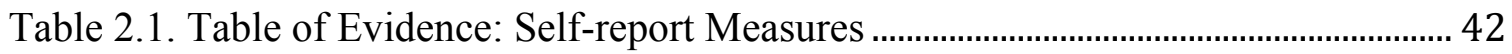

Table 2.2. Table of Evidence: Self-report Measures Psychometric Properties................... 44

Table 2.3. Table of Evidence: Performance-based Measures................................................. 47

Table 2.4. Table of Evidence: Performance-based Measures Psychometric Properties... 51

Table 2.5. New Measures of Hand and Arm Function Identified in the Review ............... 58

Table 3.1. Construct Scheme to Represent Hand and Arm Function Dimensions and

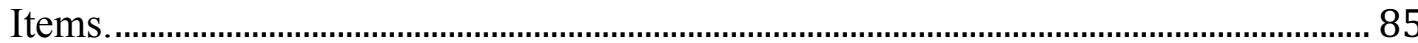

Table 3.2. Design Pattern Attributes with Corresponding Messick and Toulmin

Argument Components and Application to Hand and Arm Function Measure......... 86

Table 3.3. Comparison of Original and Revised Construct Definition, Impact of Revision on Assessment Design, and Studies to Support Evidence for Validity of Scores..... 87

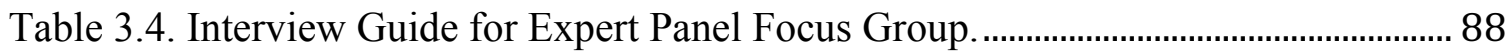

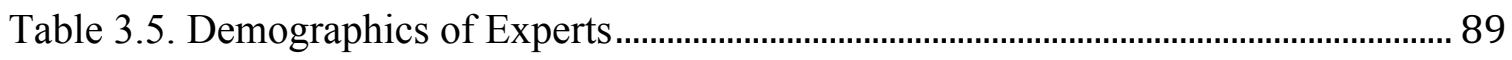

Table 4.1. Semi-Structured Focus Group Guide for Participants with Neurological

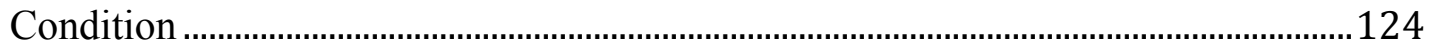

Table 4.2. Semi-Structured Cognitive Interview Guide for Participants with Neurological Condition 125

Table 4.3. List of Materials for Performance-Based Section of Hand and Arm Function

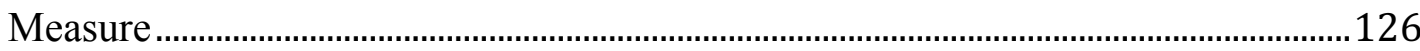

Table 4.4. Characteristics of Participants with Neurological Conditions ...........................127

Table 4.5. New Items from Cognitive Interviews ................................................................128

Table 5.1. Multitrait Multimethod Matrix with Expected Correlations ...............................142

Table 5.2. Planning for Research to Support Interpretation and Use of Assessment Scores 


\section{List of Appendices}

Appendix A. Definitions for Terms Used in this Study Related to Measurement Criteria

Appendix B. Studies Identified in the Systematic Review for the Self-report Measures

Appendix C. Studies Identified in the Systematic Review for the Performance-based

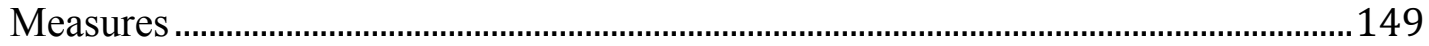

Appendix D. Item Bank with Descriptions.

Appendix E. Self-report Section of the Hand and Arm Function Measure Administered at

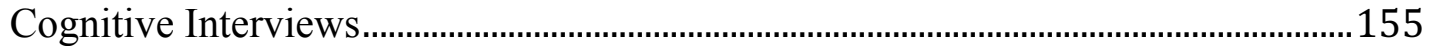

Appendix F. Performance-based Section of the Hand and Arm Function Measure

Administered at Cognitive Interviews . 


\section{Chapter 1: Introduction}

Skillful hand use is essential to daily tasks, self-management, community activities, leisure, and employment. We use our hands to grasp, hold, manipulate objects and tools, and to support our bodies (Radomski \& Trombly, 2007). We also use hands for uniquely human functions from identifying objects and gestures to sign language and reading Braille. Functions of the human hand are many and varied. The human hand function ranges from sensory to motor functions (Jones \& Lederman, 2006). It can be viewed as a continuum from tactile sensing (e.g., passive touch) to active haptic mode (e.g., perception and manipulation of objects using the senses of touch and proprioception) to prehension (e.g., tool use) to non-prehensile skilled movements (e.g., keyboarding) (Jones \& Lederman, 2006). The neural control of the hand is far more complex involving grasp and reach specialized regions in the brain along with multiple interconnections to enable cognitive, visual and emotional control of hand movements.

We need hands and we take them for granted until impairments make us aware of their true potential (Napier \& Tuttle, 1993). Hand function impairments cause difficulty participating in life roles (Al Snih, Markides, Ottenbacher, \& Raji, 2004; Radomski \& Trombly, 2007). Neurological disorders such as stroke, traumatic brain injury (TBI), Parkinson disease (PD), and multiple sclerosis (MS) cause hand function impairments (Kandel, Schwartz, Jessell, Siegelbaum, \& Hudspeth, 2012). These disorders are commonly encountered by therapists in the clinic and are the focus of this research study. Stroke and TBI are non-progressive conditions and MS and PD are progressive conditions affecting hand function. The differences in the way these disorders can affect arm and hand function is presented in Table 1.1.

After stroke, on discharge from the hospital, often arm-hand function is not recovered

(Tyson \& Turner, 1999, 2000). Four years after stroke, $67 \%$ of the patients experience the non- 
use or disuse of the affected arm as a major problem (Broeks, Lankhorst, Rumping, \& Prevo, 1999). In severe TBI, hand function deficits related to motor impairments are commonly encountered (NIH Consensus Development Panel on Rehabilitation of Persons With Traumatic Brain Injury, 1999). For people with MS, hand function impairments may be quite disabling (Feys, Romberg, Ruutiainen, \& Ketelaer, 2004) and affect their quality of life (Yozbatiran, DerYeghiaian, \& Cramer, 2008). PD and MS both can be treated with disease modifying drugs that can slow down the progression of the disease, thereby reducing the impact on hand function in daily activities.

Therapists and other professionals need hand and arm function measurement tools that are relevant to individuals with neurological condition (Kraft et al., 2014; Velozo, Seel, Magasi, Heinemann, \& Romero, 2012). The term 'measure' is defined as 'a system or scale of standard measuring units' when used in reference to hand and arm function measurement. Clinicians need reliable and valid measures of hand function so they can monitor progress, set goals, determine effectiveness of intervention, and seek reimbursement for therapy services (Carmeli, Patish, \& Coleman, 2003; Lemmens, Timmermans, Janssen-Potten, Smeets, \& Seelen, 2012). In order for a measure of hand function to be most useful for these purposes, test items need to be well developed and the scores need to be reliable and valid for the stated purpose (Lehman et al., 2011). In the words of Elaine Ewing Fess - an expert in hand therapy, author of Functional Tests in the book Rehabilitation of Hand and Upper Extremity (Skirven, Osterman, Fedorczyk, \& Amadio, 2011) - on assessment of hand,

"A thorough and unbiased assessment procedure furnishes information that helps predict rehabilitation potential, provides data with which subsequent measurements may be 
compared, and allows medical specialists to plan and evaluate treatment programs and techniques." (p. 152)

The existing hand function measures can be categorized into performance-based measures and self-reported measures. Some performance-based measures such as the Jebsen Taylor Hand Function Test (Stern, 1992) and the Nine Hole Peg Test (Oxford Grice et al., 2003) can be used for multiple diagnostic populations (Asher, 2007). Other performance-based measures are diagnosis specific such as the Wolf Motor Function Test (D. Morris, Uswatte, Crago, Cook, \& Taub, 2001) or Action Research Arm Test (Yozbatiran et al., 2008), which are used to assess hand impairments in individuals post-stroke (Velstra, Ballert, \& Cieza, 2011). More recently, self-report measures for assessing hand function have been developed such as the Motor Activity Log for survivors of stroke (Uswatte, Taub, Morris, Vignolo, \& McCulloch, 2005). Measures developed for people with stroke cannot be used in other diagnostic conditions unless there is evidence to support their use. Moreover, these currently available measures have many limitations such as small normative sample sizes, poor evidence for validity of scores at high and low end of impairments, lack of items that assess activity and participation, and lack of psychometric studies in the population of interest (Ashford, Slade, Malaprade, \& Turner-Stokes, 2008). Moreover, the performance measures require significant time for administration and may have costs associated with purchase. Thus, there is a need for the conceptualization and development of a measure of hand and arm function to overcome these limitations.

Recent research points towards the need for two components to assess hand function in the clinic: 1) perceived hand use and 2) the quality and speed of task performance (Rallon \& Chen, 2008). Currently no measure exists that has fully addressed both a performance component and a self-report component in a single measure (Asher, 2007). This dissertation 
begins the process of development of a new measure of hand and arm function, i.e., Hand and Arm Function Measure (HAFM) that includes both these components. This new measure of hand and arm function will have the potential to shed light on the importance of using self-report along with task performance. It is intended be practical, as there are a multitude of daily living tasks that can be measured via self-report but cannot be administered and tested reliably within the evaluation time frame of most clinical settings.

There are many stages in the development of a new measure. Developing an item bank is recognized as one of the critical steps in this process (D. Cook \& Beckman, 2006; K. Cook et al., 2007). Lehman et al. (2011) have conducted and documented a similar study in which they developed an upper extremity item bank for patient reported outcome measure in musculoskeletal conditions with two uni-dimensional constructs of gross upper extremity and fine hand use.

Grounding of a new measure in theory and evidence requires developing the construct or idea behind its development (Messick, 1989). Researchers in education and psychology have developed a systematic approach to guide this process called Evidence Centered Design (ECD) Framework (Mislevy, 2011; Mislevy, Almond, \& Lukas, 2003). This framework provided structure to the process of developing the HAFM. This framework suggests literature review, expert input and participant information to be utilized in the process of development of a measure before piloting the items. There are only a few hand function measures that have been developed in a systematic manner (O'Dell et al, 2013) and none that have utilized state-of-the-art measurement approaches such as ECD Framework.

The ECD framework emerged from an effort (Mislevy, 2007; Mislevy et al., 2003) to bridge the gap between the conceptualization of construct-related evidence for validity and 
practical ways in which researchers can implement the idea of supportive arguments for the interpretation and use of test scores. ECD is based on the more recent work by Samuel Messick (Messick, 1994). ECD has been used in diverse assessments such as teacher certification examinations and commercial vehicle driver assessments (Mislevy, 2011). The assessment argument is structured in layers of ECD, which are organized serially however the work continues with iterations back and forth between theory, construct development, assessment development, and evidence. The layers of ECD are as follows:

1. Domain analysis: This is the first step in assessment design where analysis involves gathering information about the domain in the context of assessment. Here, domain implies the construct or abstract idea. The analysis also goes deeper into the content, concepts, and terminology and representational forms that people working in this domain use. The knowledge representations important for the domain include knowledge, skills and attitudes that are relevant. A common way of domain analysis is task analysis, which has been practiced widely in rehabilitation science.

2. Domain modeling: This involves the conceptualization process. In domain modeling the information and relationships discovered in domain analysis are organized in a narrative form. Some examples of forms of representation of domain modeling are claims and evidence worksheets showing the relationship between what claims are to be made based on assessment scores and what evidence is gathered to support the claim. Toulmin diagrams and design patterns are other ways of domain modeling that help to structure the evidence (Mislevy et al., 2003). This step facilitates the link between design patterns, Toulmin arguments and the upcoming step of a Conceptual Assessment Framework. 
3. Conceptual Assessment Framework: This step involves writing the test and task templates, test and task specifications and scoring algorithms. Messick (Messick, 1994) suggested organizing this step in three models from the perspective of student (patient or client), evidence and task.

4. Assessment implementation: The making of the actual test that includes authoring tasks, scoring details, implementing statistical models, piloting and finalizing evaluation procedures and producing test materials and presentation environments (paper pencil versus computer based) that will be utilized.

5. Assessment Delivery: Mislevy (2007) has laid out four-process delivery architecture. This assessment delivery process is where 1) the therapist selects the task (activity selection), 2) patient/client interacts with tasks (presentation process), 3) performances are evaluated (evidence identification process), 4) feedback is created and reports are generated (evidence accumulation process).

These steps are guidelines and there is a planned redundancy in this design. For example, some decisions involved in domain modeling and conceptual assessment framework may overlap. However, this further strengthens the process and assures that the test designer carefully examines the background and nature of the construct before delivering the final product. The ECD framework also structures the terminology used for the measurement concepts. The older terms 'reliability and validity of the measure' are now appropriately modified to 'evidence for reliability and validity of scores of the measure.' This is a major change in the field of measurement brought about by the focus on evidence. Thus, ECD provides structure to the research process of developing a new measure in many different ways. 
Some other assessment frameworks have been developed in recent years such as the patient reported outcome measure development framework given by the Federal Drug Administration (FDA) (U.S. Department of Health and Human Services FDA Center for Drug Evaluation and Research, U.S. Department of Health and Human Services FDA Center for Biologics Evaluation and Research, \& U.S. Department of Health and Human Services FDA Center for Devices and Radiological Health, 2006). The steps in the FDA framework are presented in a circular flow diagram and include: 1) identify concepts and develop conceptual framework, 2) create instrument, 3) assess measurement properties, and 4) modify instrument. However, the ECD framework was selected over the FDA framework as it allows for more conceptual and domain specific development of the measure at various stages from construct to delivery; it ties all of the steps together in the diagrams that support the construct; and there is no intention to seek FDA approval. That said, the basic methods of this research align with the ECD and FDA frameworks.

The overall purpose of this research was to ultimately develop a psychometrically robust hand and arm function measure for people with neurological disorders that assesses both performance-based and self-report components. The performance-based component assesses tasks and self-report component assesses daily living items targeted towards activity and participation. This dissertation research begins the process of developing this measure for clinical use by rehabilitation professionals. Developing a new measure for arm and hand function is a lengthy process that needs a systematic approach. It is the thesis of this work that to develop a new measure of arm and hand function, qualitative information from stakeholders such as people with neurological condition, clinicians who are involved in care, and measurement experts is needed to inform item bank development. 
The objectives of this study were:

- To review the literature regarding hand and arm function and existing hand and arm function measures to inform the construct definition.

- To develop the construct definition for a new arm and hand function measure.

- To synthesize qualitative information from experts for item bank development, item writing and item modification.

- To synthesize qualitative information from people with neurological condition to develop item bank and during HAFM administration.

The chapters are organized in the order in which the research studies were conducted. Chapter 2 titled 'Literature Review' provides a background of literature related to classification of hand function, neural control of arm and hand, and a systematic review of hand function measures. Chapter 3 titled 'Construct definition and Expert Panel Study' explains the construct of arm and hand function and explains the expert panel study of the construct and item development. Chapter 4 titled 'Focus groups and Cognitive Interviews Study' explains the qualitative study involving people with neurological conditions and the process of item bank development. Chapter 5 titled 'Discussion and Future Research' presents an overarching discussion of the research studies conducted to-date, explains the process adopted for piloting of HAFM, and presents the long-term research plan for developing a psychometrically robust HAFM.

The term 'hand and arm function' in this dissertation was used generally to mean the function of the entire upper extremity and includes shoulder, arm, elbow, forearm, wrist, and finger movements for function. The terms 'hand and arm function,' 'hand function,' 'upper extremity function,' and 'upper limb function,' are used interchangeably in the literature and in 
this dissertation. In this dissertation, although every attempt was made to use the term 'measure' to refer to the assessments, some other terms such as 'test', 'questionnaire,' 'index,' 'scale,' 'tool,' 'inventory,' 'instrument,' or 'assessment' may be used interchangeably. In this dissertation, 'tool' and 'instrument' were terms used not only for mechanical measuring devices such as dynamometer, but also occasionally used for questionnaires. The use of the term 'outcome' meant the change due to the effect of a therapy, training, or due to other effects. The terminology used for describing measurement properties is defined in Appendix A.

To summarize, the development of a new measure of hand and arm function should be grounded in theory and experiences of the stakeholders to overcome the limitations of the currently available measures. The ECD framework utilized for scale development helps systematically address each area of development to achieve the ultimate purpose of developing a psychometrically robust measure of hand and arm function. 
Table 1.1. Descriptions of Arm and Hand Issues in Neurological Conditions

\begin{tabular}{lll}
\hline $\begin{array}{l}\text { Neurological } \\
\text { condition }\end{array}$ & Progression & Arm and hand issues \\
\hline Stroke & Non-progressive & $\begin{array}{l}\text { Mostly unilateral deficits with motor and sensory } \\
\text { involvement, learned non-use, low tone initially, } \\
\text { followed by spasticity. }\end{array}$ \\
$\begin{array}{l}\text { Traumatic Brain } \\
\text { Injury }\end{array}$ & Non-progressive & $\begin{array}{l}\text { Unilateral or bilateral deficits, associated vision and } \\
\text { coordination deficits, most recover fully in } 6 \text { to } 12 \\
\text { months, and/or low, high or fluctuating tone. }\end{array}$ \\
Parkinson Disease & Progressive & $\begin{array}{l}\text { Tremors on both sides early on, incoordination, slow } \\
\text { movements, involuntary movements, rigidity and/or } \\
\text { weakness. }\end{array}$ \\
Multiple Sclerosis & Progressive & $\begin{array}{l}\text { Mostly bilateral sensorimotor impairments along with } \\
\text { spasticity, fatigue and/or vision impairments. }\end{array}$ \\
\hline
\end{tabular}




\title{
Chapter 2: Literature Review
}

\begin{abstract}
Background: The human hand function has been classified in different ways to identify the unique abilities of the hand. The neural control of hand is complex and the contemporary theories on voluntary control of the hand, guide the development of the measure designed for people with neurological conditions. When developing a new measure of hand function, it is important to have an understanding of human hand function, the neural control of the hand and what hand function measures currently exist. There are many measures of hand function developed over the years. Only a few have gone through the rigor of psychometric testing and many lack evidence for validation in the neurological conditions of stroke, multiple sclerosis, traumatic brain injury and Parkinson disease.
\end{abstract}

Objective and Method: An in-depth literature review of hand function classification and neural control theories was conducted with the objective of identifying the models that would provide the theoretical foundation for the Hand and Arm Function Measure (HAFM). A systematic review of literature was conducted with the objective of identifying and describing the current hand function measures for people with neurological conditions. The secondary objective of the literature review was to map these hand and arm function measures to the aspects of hand function addressed by the measures.

Results: The literature review identified Jones and Lederman's classification as a particularly useful model for classifying human hand function as it takes into account the sensory capability of the hand. The International Classification of Function, Disability and Health (ICF) also influenced the structure of the HAFM. Both these theoretical foundations for the measure are discussed in detail in this review. The influence of the contemporary models of neural control is also discussed in depth for their role in shaping the development of the measure. The 
systematic review identified 22 hand function measures used in 86 studies in the past decade (January 2005 to July 2014). None of the measures had a combination of self-report and performance-based sections. The most commonly used performance tests were the Action Research Arm Test, the Fugl-Meyer Assessment, and the Wolf Motor Function Test (WMFT). The most commonly used self-report measures were the Motor Activity Log and the Stroke Impact Scale - Hand domain. Most of the measures lacked psychometric rigor, did not include sensory capability of the hand, and were not adequately studied in neurological conditions.

Conclusions and Significance: The new measure is grounded in the theoretical foundations of the ICF, models of classification of hand function, and contemporary neural control theories. The systematic review on hand function measures highlights the need for a new measure of hand function that is psychometrically robust, provides evidence for validation in people with neurological conditions, developed systematically, and contains self-reported and performance-based components integrated in the measure. 


\section{Introduction}

The theoretical foundation for measuring upper extremity function in neurological disorders is based on what we know about human hand function, the neural control of the arm and hand, and the measures developed in the past. A critical appraisal of existing literature on hand function tests and their psychometrics provides for an analysis of contemporary views around hand function measures. This literature review chapter is organized into: models of classification of hand function, a review of the neural control of the arm and hand including brain areas controlling movements, and a structured review of hand function measures.

\section{Models of classification of hand and arm function}

Human hand function is complex and can be classified in numerous ways. In occupational therapy (OT) literature, hand function has been broadly classified into gross motor (i.e., movements involving large muscles of the arm and hand) and fine motor function (i.e., movements involving small muscles of the hand) (Radomski \& Trombly, 2007). Also, prehension that involves finger movements and grasp that involves the use of the palm have been considered separate aspects of hand function (Pendleton \& Schultz-Krohn, 2006). The diverse range of postures that the hand can assume, have been described by Jones and Lederman (Jones \& Lederman, 2006). These postures include 16 different power and precision grasps from the original taxonomy presented by Cutkosky \& Wright for robotic hands (Cutkosky \& Wright, 1986; Venkataraman \& Iberall, 2012). Hand function has also been classified into reach, grasp, and manipulation by motor control theorists (Shumway-Cook \& Woollacott, 2011).

In the late 1970s, Sollerman and Sperling (1978) developed a handgrip classification system based on 4 finger grips (i.e., pulp pinch, lateral pinch, tripod pinch, and five-finger pinch) and 4 volar grips (i.e., diagonal volar grip, transverse volar grip, spherical volar grip, extension 
grip). Napier and Tuttle (1993) broadly classified hand function into prehensile and nonprehensile functions. Later, Jones and Lederman (2006) presented the sensorimotor continuum with the five categories that included tactile sensing, active haptic mode, prehension, nonprehensile skilled movements and transport. In motor control literature (Shumway-Cook \& Woollacott, 2011) the grasping patterns are reported to be a function of location, size and shape of the object however, in contrast Napier and Tuttle (1993) argue that the intended activity is the most important consideration. Jones and Lederman's classification that includes sensory aspects of the hand and the transport function of the arm was adopted for examination of hand function measures in the systematic review presented later in this chapter.

In Jones and Lederman's (2006) classification, tactile sensing refers to the passive detection of superficial sensation applied to the skin. Active haptic mode refers to the active movements involved in detection of properties of objects using proprioception. Prehension includes hand functions that involve various grips (e.g., power, precision, hook) and pinch along with the specialized function of opposition. Non-prehensile skilled movements include pushing, pointing, turning and carrying motions. Transport includes proximal joint functions needed for reach to mouth, overhead, forward, and back. Pediatric occupational therapy literature has further expanded the function of pinch to include in-hand manipulation (Humphry, Jewell, \& Rosenberger, 1995). In-hand manipulation is the ability to hold and move an object within one hand and is divided into three major categories: rotation, translation and shift. All Jones and Lederman's categories may include 'stabilization' which involves the ability to hold the object using the ring finger and little finger while moving an object with the thumb, index and middle fingers. Translation is the ability to move objects from the fingertips to the palm or palm to the fingertips such as moving coins to place in vending machine. Shift is the ability to move an 
object in a linear manner with the fingertips such as fanning cards in the hand. Rotation is categorized as simple and complex. Simple rotation is the ability to turn an object around the pads of the fingers and thumb. Complex rotation is the ability to turn an object end to end such as flipping a pencil from writing end to eraser end.

Many of these aspects of hand function are impaired in people with stroke, multiple sclerosis (MS), traumatic brain injury (TBI), and Parkinson disease (PD). In order to better understand and measure hand function in people with these conditions, it is helpful to have an understanding of the theoretical foundation of the neural control of arm and hand.

\section{Neural Control of Hand and Arm}

The neural control of arm and hand is not fully understood. The voluntary control of the arm and hand is different from reflexive and patterned movements of the nervous system. Models have been developed by contemporary theorists to explain mechanisms that can form the theoretical basis of the new measure. Some measures like the Fugl-Meyer Assessment were developed based on the Twitchell and Brunnstrom's concept of sequential stages of motor return in the hemiplegic stroke patient (Brunnstrom, 1966; Gladstone, Danells, \& Black, 2002) and assesses synergistic movements. The Motor Assessment Scale (MAS) was developed based on Carr and Shepherd's (Carr, Shepherd, Nordholm, \& Lynne, 1985) Motor Relearning Principles.

The new measure developed in this research study, i.e., Hand and Arm Function Measure (HAFM), is based on the current concepts in neural control of individual-task-environment model (Shumway-Cook \& Woollacott, 2011) and Shadmehr and Krakauer's (2008) model from computational neuroscience. Shumway-Cook and Woollacott (2011) describe the influence of the individual, task and environment and their interaction with each other in feedback and feedforward loops. According to this model, an individual and their central nervous system, act in the 
perception-cognition-action series to solve the problem of picking up a glass of water. The peripheral receptors in hand are involved in sensing and the primary and secondary sensory cortical areas of the brain are involved in perceiving. The parietal association areas in the brain are involved in interpreting and the pre-frontal cortex and association areas are involved in conceptualization. The supplementary motor cortex, the basal ganglia and the cerebellum are involved in developing the movement strategy and the primary motor cortex activates the action sequence. The motor neurons, muscles and joints then execute the action. Shadmehr and Krakauer's (2008) model states that an individual performs movements to achieve a rewarding state. This model accounts for the role of the subcortical structures such as basal ganglia in making an important contribution to movement. This model proposes that the individual will accomplish a given task in the most efficient strategy, path or trajectory with the least cost and most reward. Applying the concepts of this model, a predetermined path of movement for a task on a test item may not be strictly followed by the patient/client if the cost and rewards change.

These models bring the focus of the HAFM on the individual with self-reported items, the task with complexity and time elements, the environment with standardized and familiar equipment, the motor planning aspects of movement with qualitative assessment by the therapist, and the sensory aspects with active assessment combined with movement. Other than these two models, the recent research into reach and grasp regions (Buneo, Jarvis, Batista, \& Andersen, 2002; Hwang, Hauschild, Wilke, \& Andersen, 2012) in the brain, the two areas that control reach and grasp functions of the arm and hand respectively, also inform the need to test these aspects in the new measure. Thus, prehensile and non-prehensile aspects are included in the measure. 


\section{Review of Hand and Arm Function Measures}

Assessment of the upper extremity is a vital aspect of clinical evaluation for rehabilitation clinicians working with people with neurological conditions. There are numerous measures of upper extremity function in literature. However, few of them have undergone rigorous psychometric analysis to be used with confidence in research and clinic. Some of the older tests have not stood the test of time and are largely not used in the clinic, e.g., Rivermead Motor Assessment (RMA) (Lincoln \& Leadbitter, 1979). The existing literature reviews on upper extremity function measures conducted in recent years have been primarily related to stroke (Ashford et al., 2008; Ashford \& Turner-Stokes, 2013; Lemmens et al., 2012) and do not provide a clear picture of the measures used for other neurological conditions. Other reviews for diagnostic groups such as multiple sclerosis have been conducted but they are narrative reviews that include selected measures (Kraft et al., 2014). No reviews specifically targeting upper extremity measures in traumatic brain injury or Parkinson Disease have been found. Other reviews have focused on classification of the measures into the International Classification of Functioning, Disability and Health (ICF) domains and include orthopedic and neurological conditions (Metcalf, Adams, Burridge, Yule, \& Chappell, 2007; Velstra et al., 2011). One review (Velstra et al., 2011) reported frequently used upper extremity outcome measures in stroke and other musculoskeletal conditions, but the selection of outcome measures was arbitrary. Thus, in order to understand the current state of hand function measures in the selected neurological conditions, an in-depth literature search was warranted.

Objective. The objective of this systematic review was to identify and describe the commonly used upper extremity function measures for people with the neurological conditions 
of stroke, TBI, PD, and MS. A secondary objective was to map these measures to the domains of hand function targeted by the measures.

Method. Data sources. The PubMed database was searched for studies with the following keywords: 'hand,' 'arm,' 'function,' 'measure,' 'stroke,' 'multiple sclerosis,' 'Parkinson's disease,' and 'traumatic brain injury' in the following combination.

(hand AND arm) AND function AND Measure AND (stroke OR multiple sclerosis OR

Parkinson's disease OR traumatic brain injury) AND ("last 10 years"[PDat] AND

Humans[Mesh] AND English[lang])

Inclusion and exclusion criteria. The inclusion criteria were: measures for adults (19 years of age or older, pre-set in PubMed) with either acute, subacute or chronic stroke, PD, TBI or MS; full text studies in the English language published within the last 10 years (January 2005 to July 2014); and hand function measures with studies of evidence for the reliability and validity of scores. The exclusion criteria were: studies related to pediatrics and non-clinical measures such as robotic assessments and imaging studies with no clinical measures. Instrumented measures that need special measurement devices (e.g., hand held dynamometry) were excluded due to lack of information about activities of daily living. Animal studies were also excluded.

Results. PRISMA guidelines (Preferred Reporting Items for Systematic Reviews and Meta-analysis) are used for reporting of results of this review (Moher, Liberati, Tetzlaff, Altman, \& PRISMA Group, 2009). PubMed database search yielded 129 studies. After screening title and abstract, three studies related to pediatrics and 40 studies that did not meet criteria for hand function measures were excluded. Eighty-six studies were selected for full text review and of these 34 hand function measures were identified. Ten measures that were newly developed with limited or no evidence for the reliability and validity of scores and two instrumented measures 
(i.e., Jamar® dynamometer and Pinchmeter) were then excluded from detailed analysis. This exclusion was based on the initial criteria set for the measures. Figure 2.1 presents the PRISMA flow diagram. A total of 22 measures out of 34 were selected for detailed description in this review. Seven newly developed measures of hand and arm function will be discussed briefly.

Data were extracted from the available studies related to hand function measures in general and psychometrics in particular. Cross references for hand function measures reporting development, evidence for the reliability and validity of scores were used for the purpose of constructing detailed descriptions of the measures in this review. These upper extremity function measures are subsequently described based on their construct, hand function (i.e., prehensile, non-prehensile, transport, tactile sensing and active haptic mode), ICF domain, sample item and response categories, description of population that these measures were developed for and the neurological population for whom they have been used (e.g., stroke, MS, PD and TBI), psychometric properties (e.g., evidence for the reliability, validity and responsiveness of scores), burden of administration, and the number of studies identified in the review that have used the measure.

Through this systematic review 22 hand function measures were identified which were self-report measures and performance-based measures. In the following results, the self-report measures will be reported followed by performance-based measures, along with a brief overview of newly developed measures.

Self-report measures. Seven out of 22 hand function measures found in this review were self-report measures. The details of these measures are outlined in Table 2.1 and 2.2. The most commonly used self-report measures were the MAL (17 studies) and the Stroke Impact Scale Hand Domain (SIS-HD) (11 studies). The seven self-report measures are presented in the 
following section from older to newer measures based on the first published study found in the literature.

Disabilities of Arm Shoulder and Hand (DASH). The DASH (Hudak, Amadio, \& Bombardier, 1996) is a self-report measure designed to evaluate disorders of the upper limbs and monitor change of function over time. This instrument was developed by the Institute for Work and Health and the American Academy of Orthopedic Surgeons with a systematic two stage process of item generation and item reduction (Hudak et al., 1996). The first stage of development involved input from clinicians and patients and a review of 13 relevant outcome measures in musculoskeletal conditions. The second stage involved item reduction by three content experts, secondary review by a panel of 15 experts for content and face validity evidence and item importance along with pre-testing on 20 individuals with upper extremity musculoskeletal problems (Hudak et al., 1996). Further item reduction was conducted by psychometric analysis. This 38 -item scale includes optional eight high-performance items and also has a shorter version (Quick DASH) with 11 items (Mehta, Macdermid, Carlesso, \& McPhee, 2010). It can take anywhere from 5 to 30 minutes to complete depending on the type of scale and requires no training. The difficulty with hand function over the past week was evaluated in the measure and patients/clients were asked to rate their best estimate of performance if the activity was not performed over the past week. The wording for response category of each type of item varies with the nature of the item (e.g. symptoms ask about severity or work related tasks ask about difficulty). The measure cannot be scored if more than three missing items exist.

The DASH questionnaire asks about symptoms as well as the ability to perform certain daily activities. DASH includes activities related to sensation (e.g., tingling), transport (e.g., 
reach), prehension (e.g., heavy and light) and non-prehensile (e.g., pushing and carrying) tasks. The hand function aspects not addressed by this measure are active haptic sensing, in-hand manipulation, and quality and speed of task performance. The DASH assesses difficulty on a 5point scale with daily activities for unimanual and bimanual tasks irrespective of how the task is done. Strong psychometrics, cultural adaptations and iterations to develop the Quick-DASH have been implemented (Kennedy et al., 2013) for musculoskeletal conditions. The DASH was the only measure that included the work and recreational tasks as separate modules.

The DASH has excellent evidence for reliability of scores (i.e., test-retest, inter-rater and internal consistency) in other upper limb conditions but this has not been established for neurological conditions. Face and content-related evidence for validity of scores was found to be poor specifically in stroke and not established for other neurological conditions (Lannin, McCluskey, Cusick, Ashford, \& Ross, 2010). In spite of being a well-developed measure for arm and hand musculoskeletal conditions, the DASH was reported in only three out of the 86 studies found in this systematic review. One of the major reasons for this lack of use in neurological conditions is the absence of research studies to provide evidence of validity of the scores of the measure with patients/clients with neurological conditions (Baker 2015). In addition, the DASH cannot differentiate function on right or left side. People with neurological conditions have unequal distribution of deficits and it is vital to evaluate the ability of each side separately particularly related to symptoms.

ABILHAND. The ABILHAND (Penta, Thonnard, \& Tesio, 1998) is a self-report measure of manual ability for adults with upper limb impairments. It measures a person's ability to manage daily activities that require the use of the upper limbs. The ABILHAND has been validated in chronic stroke (age 16-80) and is available in English, French, Dutch, Italian and 
Swedish (Penta et al, 1998, 2001). The ABILHAND was reported in two out of 86 studies in this review. This measure was developed in Sweden and has not been used extensively in research. It is an exclusively bimanual measure with 23 daily activities that focus on object handling (i.e., prehensile movements) and takes about 10 minutes to administer (Alt Murphy, Resteghini, Feys, \& Lamers, 2015). It has items such as threading a needle that are potentially not appropriate for people with neurological conditions. The non-prehensile and transport functions of the hand are not assessed by this measure. The ABILHAND assesses the difficulty with hand function on a 3point scale irrespective of the time frame such as past week or past month. The ABILHAND uses modern Item Response Theory (IRT) techniques of Rasch analysis to provide a score if data is entered in the online portal. Paper based version is available for download along with detailed instructions. Each activity is weighted by logits on the Rasch scale for its online version. A logit is a unit of measurement on an interval level of measurement to report relative differences between hand function ability estimates and item difficulties. Evidence for internal consistency is established and order of difficulty of items has been confirmed by Rasch analysis (Penta, 2015; Penta, Tesio, Arnould, Zancan, \& Thonnard, 2001; Simone, Rota, Tesio, \& Perucca, 2011). No studies were found that reported on intra-rater, inter-rater and test-retest evidence for reliability of scores of the ABILHAND. Concurrent and criterion-related evidence for validity of scores has been established by Penta et al (2001) and Simone et al (2011), along with evidence for responsiveness of the measure. Face validity evidence is not reported.

Motor Activity Log (MAL). The MAL (van der Lee et al., 1999) is a semi-structured interview to assess arm function exclusively for people with stroke and was developed as part of the constraint induced movement therapy research trials. It is a commonly used measure identified in 17 out of 86 final studies in this systematic review. Individuals are asked to rate 
quality of movement (QOM) (also known as How Well Scale) and the amount of use (AOU) during daily functional tasks. It has unimanual and bimanual tasks related to prehensile, non prehensile, and gross motor functions related to transport. The original version has 30 tasks and versions with 45, 28 or 14 tasks are also available. The MAL is scored on a 6-point ordinal scale from 0 (weak arm was not used) to 5 (weak arm used as well as before stroke) (Uswatte et al., 2005). It was developed for assessing real world hand use during trials for constraint induced movement therapy and psychometric studies are incomplete as the population studied is restricted to people enrolled for the clinical trials. Although the internal consistency and testretest evidence for reliability of scores were well established, the minimal detectable change and minimal clinically important difference were not reported, reducing the overall utility of the scores of this measure.

Stroke Impact Scale- Hand Domain (SIS-HD). The SIS (Duncan et al., 1999) was developed as a comprehensive measure for assessment of health status following stroke with hand function domain as one of the eight domains assessed. This is a commonly used measure and was included in 11 out of 86 studies reviewed in this systematic review. Five items in the hand section on this measure include carrying heavy objects (i.e., bag of groceries), turning a doorknob, opening a can or jar, tying a shoe lace, and picking up a dime. There are other items not in the hand domain section of this measure that are related to hand function, for example cutting food with a knife and fork, dressing the upper body, bathing, clipping toenails, and completing light household chores. These items are prehensile movements and non-prehensile (e.g., carry) functions. Transport functions such as reach are not directly addressed in the measure. The items are rated by difficulty on a 5 -point scale $(1=$ Could not do at all, $5=\mathrm{Not}$ difficult at all) and are related to performance on a typical day. Psychometric properties of SIS 
have been studied for the overall measure and for hand function domain specifically (Duncan et al., 2002). The overall scale has excellent evidence for test-retest and inter-rater reliability of scores (Carod-Artal, Ferreira Coral, Stieven Trizotto, \& Menezes Moreira, 2009; Duncan et al., 2002). The SIS-HD has adequate concurrent evidence for validity of scores and can discriminate between degrees of stroke severity with adequate correlations with Barthel Index and functional status (Carod-Artal et al., 2009). The measure is sensitive for minor and moderate stroke from 1 to 3 months and 1 to 6 months post-stroke (Duncan et al., 1999). Floor and ceiling effects have also been established (Duncan et al., 1999). In spite of extensive research into psychometric properties, face and content-related evidence for validity of scores for the hand domain have not been satisfactorily reported. The SIS-HD in itself is not a comprehensive measure of hand function for tracking progress due to the lack of hand function items and a scatter of items related to hand in other domains. Further, the SIS-HD was specifically developed for people with stroke (Duncan et al., 1999; van der Lee, Beckerman, Knol, de Vet, \& Bouter, 2004) and not studied in other neurological conditions.

Manual Ability Measure (MAM). The MAM (Chen, Granger, Peimer, Moy, \& Wald, 2005) is an occupation-based and patient-centered outcome tool that measures manual ability using a self-report format. The MAM was reported in one out of 86 studies in this systematic review. The measure was developed by filtering items from the DASH, TEMPA, ABILHAND and Sollerman Hand Function Test (Chen, 2005). The MAM has 16, 20 or 36-item formats. It includes unimanual and bimanual tasks with primarily prehensile movements related to daily activities. It measures item difficulty on a 4-point scale ( $1=\mathrm{I}$ cant do, $4=$ Easy). It has a NA (Not applicable) rating that is used when a person never did the task even before his/her hand condition. The Rasch Rating Scale Analysis method was used to develop and refine the 
instrument. Therapist input was obtained for scale development however patient input is lacking in item development. There is no time frame specified for the patient to reference when answering questions. The MAM (36-item version) was developed for musculoskeletal conditions and later validated for people with neurological conditions including stroke (Chen \& Bode, 2010). Although, the construct-related evidence for validity of scores for this measure is studied, it is limited by small sample size overall with two studies found for psychometrics. The evidence for reliability of scores, measurement error, and interpretability of the scores has not been reported.

NeuroQOL-UE. The NeuroQOL (Cella, 2015) is a self-report of health related quality of life. Upper extremity function is one of the 17 domains included in this measure. The NeuroQOL was reported in one out of 86 studies in this systematic review. This is the only measure currently known to be available in Computer Adapted Testing (CAT) format. The measure consists of fine motor and ADL items in the CAT format with variable number of items. Paperbased short forms (8-10 items) are also available across all domains. The items are rated on a 5point scale based on intensity. Scoring is via computer or can be calculated using tables in the NeuroQOL user manual for each short form. Items have been targeted to people with neurological conditions however the NeuroQOL lacks the sensitivity to change, reducing its utility in the clinic (Kraft et al., 2014). Psychometrics have been established for the NeuroQOL as a generic measure and no studies specifically related to the UE domain were found. With the available psychometrics, it is unclear if using the UE domain by itself will be beneficial for neurological population. Information presented in Table 2.1 and 2.2 is generic data (not specific to upper extremity function sub-section) from NeuroQOL final report (Cella, 2015). 


\section{Patient Reported Outcome Measurement Information System ${ }^{\circledR}$ Physical Function Bank}

for Upper Extremity (PROMIS $\left.{ }^{\circledR}-U E\right)$. The PROMIS ${ }^{\circledR}$ - UE (Hays et al., 2013), funded by the National Institute of Health (NIH), is a self-reported measure of upper extremity function developed using mixed qualitative and quantitative methods. This measure was reported in 2 out of 86 studies in this review. The upper extremity function short form contains 16 items targeting ADL tasks including unimanual and bimanual activities rated on a 5-point scale of difficulty. Psychometric studies have been done on diverse populations that include people with neurological conditions. Concurrent evidence for validity of scores with DASH $(r=0.726)$ for the PROMIS® physical function domain has been established. However, it has shown limited sensitivity to change and lacks utility in the clinical population (Kraft et al., 2014). The upper extremity short form has limited research to support its use independently. Moreover, focused research for its use with specific neurological conditions has not been conducted for this measure.

Therapists and other clinicians have traditionally relied on performance. Self-report measures have evolved in recent years with the Disabilities of Arm Shoulder and Hand (DASH) being the first self-report measure (Hudak et al., 1996) in hand function. Other measures such as the ABILHAND (Penta et al., 1998), the MAL (Kunkel et al., 1999), the SIS-HD (Duncan et al., 1999) and the MAM (Chen et al., 2005) were soon developed. The most recently developed selfreport measures, the Neurological Quality of Life - Upper Extremity Subsection (NeuroQOLUE) (Gershon et al., 2012) and the PROMIS ${ }^{\circledR}$ - UE (Tyser et al., 2014), were developed using the item response theory (IRT) (i.e., Rasch analysis). These two measures were developed by National Institute of Health (NIH) with its recent commitment to patient reported outcomes (Quatrano \& Cruz, 2011). 
All of these measures target the ICF domain of Activity and Participation. The DASH is the only measure that has attempted to address body structure and function domains by including questions related to pain, weakness, tingling, stiffness and weakness. None of the self-report measures covered other important aspects of body structure and function that address symptom burden, such as spasticity, for people with neurological conditions. Of the 7 measures, 4 were specifically developed for upper extremity function (i.e., DASH, MAM, MAL, ABILHAND) and 3 measures (i.e., SIS-HD, NeuroQOL-UE, PROMIS ${ }^{\circledR}-\mathrm{UE}$ ) include hand function within the context of a broader health assessment.

Further details of self-report measures are presented in Table 2.1 and psychometric properties in Table 2.2. The studies identified in this review that have used the self-report measures are detailed in Appendix B.

Performance-based measures. Fifteen out of the 22 hand function measures that have been researched extensively are performance-based. The details of these measures are presented in Table 2.3 and 2.4. The most commonly used performance measures were the Action Research Arm Test (ARAT) (34 studies), the Fugl-Meyer Assessment (FMA) (34 studies) and the Wolf Motor Function Test (WMFT) (11 studies). The performance-based measures are presented in the following section from older to newer measures based on the first published study found in PubMed.

Jebsen Hand Function Test (JHFT). The JHFT is also referred to as the Jebsen-Taylor Hand Function Test in literature (Beebe \& Lang, 2009). The JHFT assesses broad range of unimanual hand function required for activities of daily living and is widely used in clinics across different diagnostic categories (Jebsen, Taylor, Trieschmann, Trotter, \& Howard, 1969). The JHFT was reported in 4 studies out of 86 in this review. It has 7 unimanual subtests that can 
be completed in 15 minutes. The measure can be assembled with the detailed instructions provided in original study (Jebsen et al., 1969) however in recent years a standardized tool kit has been made available for purchase. Scoring is based on time of completion of tasks and norms have been established for dominant and non-dominant hand. The time is summed across all tasks and each task can go up to 120 seconds. The JHFT only scores time and does not include the quality of movement in its scoring criteria. The JHFT was primarily developed in orthopedic trauma where unimanual impairments were prevalent. Psychometric studies in stroke are few (Beebe \& Lang, 2009; Ferreiro, dos Santos, \& Conforto, 2010) and have small sample sizes. The content-related evidence for validity of scores in people with neurological conditions is lacking due to its reliance on unimanual tasks and speed with no recognition of task completion or quality of movement.

Rivermead Motor Assessment Upper Limb Section (RMA - UE). The RMA (Lincoln \& Leadbitter, 1979) is a motor assessment for use in people post stroke to examine upper limb, lower limb and gross function. The RMA takes 45 minutes to administer and items are arranged in order of difficulty with 3 attempts allowed for each item. Only one study out of 86 reported using RMA in this systematic review. The RMA-UE assesses gross and fine movements and daily activity tasks using 15 items. It has unimanual and bimanual tasks. Each item is scored as a 0 if they can't perform it or a 1 if they can perform it. Even though this binary scoring system makes the scale reliable, it calls to question the utility of scores in the clinic. It is not clear how the varied range of functional levels can be represented with a 0 or 1 option for responses keeping the measure at a reasonable length of 15 items. The RMA gross motor function section can be a self-report to save therapist time (Sackley \& Lincoln, 1990) however this feature has not been examined for RMA-UE. Evidence for reliability of scores for test-retest has been 
established for RMA-UE $(r=0.88)($ Lincoln \& Leadbitter, 1979) however intra-rater and interrater evidence for reliability of scores is lacking. Although unidimensionality (Lincoln \& Leadbitter, 1979) and internal consistency (Kurtaiş et al., 2009) for the RMA-UE has been established, it lacks convergent evidence for validity of scores (Kurtaiş et al., 2009).

Motricity Index - UE Subscale (MI-UE). The MI-UE (Demeurisse, Demol, \& Robaye, 1979) is a measure of movement for upper and lower extremity post stroke. This measure was reported in 3 out of 86 studies in this systematic review. This measure was developed (Demeurisse et al., 1979) for the clinic and the only equipment required is a $2.5 \mathrm{~cm}$ wooden cube. This was done intentionally to keep the burden low on the clinician for assembling and gathering the test items. The MI-UE has 3 items related to pinch grip using a cube for picking up and placing, elbow flexion, and shoulder abduction. No ADL items are included in this measure. Other than the advantages of ease of use with only 3 items and correlation with grip dynamometry (Collin \& Wade, 1990), this measure is not comprehensive and lacks the psychometric properties for use in neurological conditions.

Action Research Arm Test (ARAT). The ARAT (Yozbatiran et al., 2008) was developed from the Carol's Upper Extremity Test (Carroll, 1965; Lyle, 1981) and is available for purchase as a standardized tool or can be constructed with the specifications provided by Yozbatiran (2008). The test forms are freely available. The ARAT is a commonly used measure and was included in 34 out of 86 studies in the systematic review.

The ARAT consists of unimanual performance tasks organized in 4 subtests of grasp, grip, pinch and gross movements. However, it has been critiqued for its lack of a theoretical model in development (Finch, Brooks, Stratford, \& Mayo, 2002). This 19-item measure uses a response rating on a 4-point scale and has a standardized scoring protocol. The test takes 10 
minutes to administer. All items need not be administered if the individual does not pass the first item within a subtest. This test is unique in its ability to differentiate between grasp versus grip related tasks where grip is identified as a power grip with strength. The ARAT has the most psychometric research related to stroke, with evidence for reliability of scores studied for multiple neurological diagnoses. All of the evidence for the reliability and validity of scores reported have excellent values except poor effect size in acute stroke and high floor effect in acute stroke at 14 days with $41.5 \%$ of participants experiencing the floor effect (Lin, et al., 2009).

Fugl-Meyer Assessment - Upper Extremity Subscale (FMA-UE). The FMA-UE evaluates and measures upper extremity recovery post stroke and is the most widely used measure in stroke trials (Gladstone et al., 2002) and was reported in 34 out of 86 final studies in this systematic review. It is the only sensorimotor measure of function that includes joint pain (selfreported) measurement during passive motion, however the item has been critiqued for its representation of arthritic conditions causing joint pain versus stroke causing muscle related pain (Gladstone et al., 2002). The FMA-UE measures reflexes, movement synergies, and volitional movement. Performance is rated on a 3-point scale of none, partial and full. Items in the motor domain have been derived from Twitchell's 1951 description of the natural history of motor recovery following stroke and integrates Brunnstrom's stages of motor recovery (Gladstone et al. 2002; Poole \& Whitney, 2001). Items of the FMA are intended to assess recovery within the context of the motor system. Functional tasks are not incorporated into the evaluation (Chae, Labatia, \& Yang, 2003). A major criticism of the FMA is that it is a lengthy measure to administer and joint pain domain does not fit with the construct of the scale (Gladstone et al., 2002). Sometimes it takes longer than 35 minutes to complete, such as when it is administered to 
patients who are aphasic or severely affected (Kusoffsky, Wadell, \& Nilsson, 1982; Dettmann, Linder, \& Sepic, 1987). Most aspects of evidence for reliability of scores have been established for the FMA and specifically for the FMA-UE except the intra-rater evidence for reliability of scores. There have been detailed studies related to evidence for validity of scores of the measure in severity of stroke for acute, subacute, and chronic stages and is highly recommended by many of the outcome measure working groups (e.g., StrokEDGE group of American Physical Therapy Association).

Functional Test of Hemiplegic Upper Extremity (FT). The purpose of the FT is to evaluate the hemiparetic or hemiplegic arm's motor capability for function (Wilson, Baker, \& Craddock, 1984). Fong et al. (2004) has adapted the FT for use in Hong Kong. The FT was reported in one out of 86 studies in this review. It consists of 17 graded activities that are arranged in 7 levels of graded degree of difficulty according to Brunnstrom's stages of stroke motor recovery. The tasks range from resisted contralateral muscle contractions to elicit associated reactions such a elbow or shoulder flexion in the impaired arm to tasks requiring a high degree of upper extremity coordination and finger dexterity of the impaired arm such as putting a light bulb into a socket held at shoulder height. The FT takes about 30 to 90 minutes to administer. The psychometric research is limited to inter-rater evidence of reliability and concurrent evidence for validity of scores only, limiting its utility for use as an outcome measure.

Box and Blocks Test (BBT). The BBT (Mathiowetz et al., 1985) is a test of unimanual gross manual dexterity with standardized equipment available for purchase. It was reported in 6 out of 86 studies in this review. It is a short test and takes about 2-5 minutes to administer. The test assesses the ability to pick up a 1-inch cube one at a time and move it across the box over a barrier within one minute. It is also a measure of endurance for repetitive tasks over time. 
Multiple studies of the BBT have been conducted in neurological populations. Test-retest and inter-rater evidence for reliability of scores is excellent along with good concurrent evidence for validity of scores with the NHPT and the ARAT (Lin, Fu, et al., 2010). The BBT has also been reported as the best predictor of upper limb function at 5 weeks post-stroke (Higgins, Mayo, Desrosiers, Salbach, \& Ahmed, 2005). The BBT tests only one aspect of hand function for one type of object and is best used in combination with other functional metrics for tracking hand function. Thus, it is not a comprehensive test of upper extremity function and does not include daily activity tasks.

Nine Hole Peg Test (NHPT). The NHPT (Heller et al., 1987) is test of finger dexterity administered by asking the client to take the pegs from a container one by one and place them into holes on the board as quickly as possible. Participants must then remove the pegs from the holes, one by one and replace them in the container. Scores are based on the time taken to complete the activity recorded in seconds. Alternatively the NHPT can be scored based on the number of pegs placed in 50 or 100 seconds (Sunderland, Tinson, Bradley, \& Hewer, 1989). This measure was reported in 7 out of 86 studies in this review and has been validated in people with neurological conditions (Heller et al., 1987). Pinch and in-hand shifting are the only dexterity aspects assessed by this measure. Most of the psychometric studies indicate strong evidence for reliability and fair concurrent evidence for validity of scores (Erasmus et al., 2001; Sunderland et al., 1989). However, the two versions with 50 and 100 second limit have not been validated separately with 100 second being the typical method (Sunderland et al., 1989). Poor predictive evidence for validity of scores, unknown face and content-related evidence for validity of scores, ceiling effects and Minimal Clinically Important Difference (MCID) reduce the utility of this measure. 
Motor Assessment Scale (MAS). The MAS (Carr et al., 1985) assesses eight areas of motor function. Three trials of each item are administered but only the best performance is recorded. Three out of the 8 areas are specifically related to the upper extremity and includes upper arm, hand movements and advanced hand activities of object manipulation. This measure was reported in 4 out of the 86 studies in this review. The MAS uses a 7-point scale of motor behavior for each area hierarchically organized from beginning to end stage of recovery. It takes about 15 minutes to administer the entire measure. The MAS targets the hand function domains of prehension, transport and non-prehensile skilled movements. Although preliminary psychometrics have been established for this measure (English, Hillier, Stiller, \& Warden-Flood, 2006; Malouin, Pichard, Bonneau, Durand, \& Corriveau, 1994; Tyson \& DeSouza, 2004), no evidence for validity or reliability of scores exists specifically for using the upper extremity items of the MAS independently. Only one study (English et al., 2006) reported evidence for responsiveness of the arm items with $80 \%$ of subjects rating at the extremes of the scale and demonstrating poor evidence for responsiveness of the MAS - UE. Floor and ceiling effects ( $18 \%$ for upper arm function, $41 \%$ for hand movements and 47.5 for advanced hand activities) have been reported for the 3 upper extremity items (English et al., 2006) calling in to question the overall utility of the measure for hand function.

Sollerman Hand Function Test (SHFT). The SHFT (Sollerman \& Ejeskär, 1995) is a 20item objective measure designed to assess hand function. This measure was reported in one out of 86 studies in this review. The patient/client is given 60 seconds to complete each item and consists of a 5-point rating scale. The scale consists of items that target seven different hand grips and consists of a box with 20 items such as lock, zipper, cubes, screwdriver, etc. It targets the hand function domains of prehension and transport. This test has test-retest and inter and 
intrarater evidence for reliability of scores established for stroke with no evidence for validity of scores (Brogårdh \& Sjölund, 2006; Weng et al., 2010). For tetraplegia and gout, there is concurrent and convergent evidence for validity of scores reported (Dalbeth et al., 2007; Sollerman \& Ejeskär, 1995). There is no research regarding the measure's psychometric data for evidence of internal consistency, responsiveness, minimal clinically important difference (MCID), or floor and ceiling effects.

Arm Motor Ability Test (AMAT). The AMAT (Kopp et al., 1997) evaluates disabilities in upper extremity function in activities of daily living using a quantitative and qualitative measure. This test is unique in its breakdown of $13 \mathrm{ADL}$ activities into 1 to 3 component tasks or movement segments. During the AMAT assessment, the patient/client is not aware of the analysis of each individual segment and goes through the natural flow of movement. Each task is timed and rated according to quality of movement and ability to perform each component of the task. Tasks have a 1 to 2 minute performance time limit. It is a lengthy measure to administer. It has 10-item (28 component tasks) and 9-item (without the light switch/door task) versions. It is scored on a 6-point scale from 0 (does not attempt to use affected arm) to 5 (movement appears normal). This test evaluates prehensile (grip small and large object, pinch), non-prehensile and transport functions of the hand and arm. It has adequate test retest and inter-rater evidence for reliability of scores and concurrent evidence for validity of scores (Chae, Labatia, \& Yang, 2003; Kopp et al., 1997). However, only two reported studies of this measure were available and there is not sufficient study of the psychometric properties using multiple studies for this measure.

Wolf Motor Function Test (WMFT). The WMFT (Hsieh et al., 2009) was developed for the constraint induced movement therapy trials and includes a quantitative test of upper extremity motor ability through timed and functional tasks. This is a commonly used test 
included in 11 out of 86 studies in this systematic review. It was originally comprised of 21 items however the most commonly used version has 17 items that includes 15 function-based tasks and 2 strength based tasks. The 21 -item version has 3 parts, timed tasks (tasks 1-6), functional ability (tasks 1-6, 15-21) and strength (tasks 7-14) including dynamometry for grip strength. The examiner must test the less affected extremity first and scoring is on a 6-point ordinal scale that assesses the involvement of the more affected hand from 0 (does not attempt with the involved arm) to 5 (arm does participate, movement appears to be normal). It allows for 120 seconds ( 2 minutes) per task. The evidence for the reliability and validity of scores of the WMFT has been studied extensively in stroke (Lang, Edwards, Birkenmeier, \& Dromerick, 2008) and there is one study for TBI (Shaw et al., 2005). Overall given its strong psychometrics and its use in constraint-induced movement therapy trials, the WMFT has gained widespread use. However, since this measure was developed for research purposes, video recording of the WMFT is recommended which typically is not feasible in the clinic. Scoring accuracy is limited without video recording as a therapist might not observe all elements of the task, especially if it is done quickly or in a busy clinic.

Test Evaluant la Performance des Membres Supérieurs des Personnes Agées (TEMPA). The TEMPA (Desrosiers, Hébert, Bravo, \& Dutil, 1995)was developed for the elderly population with a theoretical framework of aging hand for test development. It includes 4 unimanual and 5 bimanual tasks related to strength, coordination, dexterity and various types of prehension. The TEMPA also takes into account speed, functional rating and task analysis in scoring. Functional rating is based on a 4-point ordinal scale. Task analysis consists of range, strength, control, prehension and fine movement. Grip strength assessment using hydraulic Jamar ${ }^{\circledR}$ dynamometer accompanies the test. Normative data for young adults (20- 44 years) is included in manual 
(Desrosiers, Hébert, et al., 1995). Concurrent evidence for validity of scores has been established in MS only and needs further research to obtain evidence for internal consistency, responsiveness and evidence for validity of scores in other neurological conditions (Feys, Duportail, Kos, Van Asch, \& Ketelaer, 2002).

Stroke Rehab Assessment of Movement Scale (STREAM). The STREAM (Daley, Mayo, \& Wood-Dauphinée, 1999) is a comprehensive measure designed for use by physical therapists for a quantitative evaluation of motor functioning after stroke. It was designed to be easy to administer in a clinical setting. It consists of upper limb (scored on a 3-point ordinal scale), lower limb (scored on a 3-point ordinal scale) and basic mobility items (scored on a 4-point ordinal scale). Extensive evidence for the reliability and validity of scores are available for this measure with overall strong psychometrics to support its use in the clinic. The upper extremity STREAM (STREAM-UE) consists of 10 items that are interspersed between the other items (Daley et al., 1999). There are no prehensile items. Movement items are assessed as the patient/client is taken in different positions from supine to sitting to standing. The lack of object handling items is a limitation of the measure.

Chedokee Arm and Hand Inventory (CAHAI). The CAHAI (Barreca, Stratford, Lambert, Masters, \& Streiner, 2005) is an exclusively bimanual upper limb measure of the functional ability of the paretic arm and hand after a stroke. The CAHAI is available in 7,8, 9, or 13-item versions. The items can be assembled with instructions and training manual from the website (www.cahai.ca). The CAHAI consists of functional bimanual tasks where role of the affected hand during the functional tasks is rated on a 7-point scale of independence (similar to scoring in Functional Independence Measure). It is a newer measure with minimal testing with only 3 studies found for evidence for the reliability and validity of scores. 
As occupational therapy (OT) emerged as a profession, the treatment areas were primarily work conditioning and hardening. This led to the development of hand function tools such as the Minnesota Rate of Manipulation Test (MRMT) (Drussell, 1959) and the Purdue Pegboard Test (PPT) (Rapin, Tourk, \& Costa, 1966) that provided an objective assessment of hand function (e.g., turning, placing, picking tasks) needed for work environment with emphasis on time and repetition. As the role of occupational therapist included treatment of war veterans with hand injuries, strength and dexterity measures such as dynamometry (Kellor, Frost, Silberberg, Iversen, \& Cummings, 1971) and functional hand measures were included. In the past 30-40 years the role of OT has expanded to include hand issues in people with disabilities related to varied orthopedic and neurological diagnoses (Radomski \& Trombly, 2007). The Jebsen Hand Function Test (JHFT) is one of the oldest (Jebsen et al., 1969) measures published that has a functional upper extremity measure and continues to be the most widely used for all hand function impairments. Although bimanual measures such as the RMA (Lincoln \& Leadbitter, 1979) were later developed, initially there was a heavy focus on unimanual assessment of the upper limb as evidenced by measures such as the Motricity Index - Upper Extremity Subscale (MI- UE) (Demeurisse, Demol, \& Robaye, 1980), the ARAT (Lyle, 1981), the Box and Block Test (BBT) (Mathiowetz et al., 1985), and the NHPT (Mathiowetz et al., 1985). As principles of neurodevelopmental therapy were developed, measures such as the FMA - UE (1983) (Berglund \& Fugl-Meyer, 1986) gained popularity with assessment of movement synergies included in the test. With increased focus on bimanual activities, measures such as the CAHAI (Barreca et al., 2005) were developed that primarily targeted bimanual function in stroke. An increasing number of measures have been developed for people with stroke, however, they have limited evidence for validity of scores across other neurological conditions. 
The 15 performance measures primarily target ICF domain of activity and include everyday tasks involving upper extremities. Of these, five measures also have movement and strength measures directly addressing body function (i.e., MI-UE, FMA-UE, Functional test of hemiplegic upper extremity [FT], Motor Assessment Scale [MAS], STREAM-UE). Out of the 15 measures, there were ten measures that were specifically developed for upper extremity function (i.e., JHFT, ARAT, FT, BBT, NHPT, Sollerman Hand Function test [SHFT], Arm Motor Ability Test [AMAT], WMFT, Test d'Evaluation des Membres Supérieurs des Personnes Agées [TEMPA], CAHAI). The other five measures (i.e., FMA, STREAM, MAS, MI, RMA) evaluate hand function within the broader context of motor assessment and include lower limb and trunk related items. Only some of the upper extremity subsections like the FMA-UE were separately involved in psychometric testing, apart from their parent test.

Further details of performance-based measures are presented in Table 2.3 and psychometric properties in Table 2.4. The studies identified in this review that have reported the performance-based measures are detailed in Appendix C.

New hand function measures. This review found seven new hand function measures that have been developed in the last ten years (see Table 2.5). There were three self-report measures, the Arm Activity Measure (ArmA) (Ashford, Turner-Stokes, Siegert, \& Slade, 2013), the Reaching Self-efficacy (Chen, Lewthwaite, Schweighofer, \& Winstein, 2013), and the Hand Function Survey (Blennerhassett, Avery, \& Carey, 2010). The ArmA (Ashford et al., 2013) was developed to separately assess the active and passive components of the affected arm. The Bilateral Arm Reaching Task (Chen et al., 2013) involves reaching self-efficacy and combines self-report and performance, wherein the participant reports confidence in reaching after 
performing a task. The Hand Function Survey is a self-report questionnaire that probes affected hand use in daily living tasks.

Four new performance measures were identified, the 16-Hole peg test (Hammer \& Lindmark, 2010), the Extended Drawing Test (Vuillermot, Pescatore, Holper, Kiper, \& Eng, 2009), the Manual Function Test (Miyamoto, Kondo, Suzukamo, Michimata, \& Izumi, 2009), and the Upper Body Dressing Scale (Suzuki et al., 2008) . The 16-Hole peg test is also known as the Functional Dexterity Test and is similar to the NHPT except that the measure only requires turning over of 16 pegs using a three-jaw chuck prehension. The Extended Drawing Test, is a drawing measure. It is known as a graphonomic test that uses computerized recording of the drawings using two tools, a graphic pen with puck-like penholder and a graphic regular-grip writing pen. The Manual Function Test, developed in Japan, has 32 items and includes arm movements and manipulative activities. The Upper Body Dressing Scale for buttoned shirt is a performance assessment of donning a buttoned shirt and scores are given for each part of the task.

Among the new measures, two measures assessed bimanual function (i.e., Reaching Selfefficacy Scale and Upper Body Dressing Scale for buttoned shirt). All of the other measures focused on unimanual function and sometimes only the function of the affected hand (i.e., ArmA). The new measures of hand function attempt to reduce the time needed for assessment by developing short measures or using modern technology such as graphonomics (i.e. Extended Drawing Test). However, the measures lack assessment of technology related daily tasks performed by patients such as using a keyboard.

Discussion. There are limitations to this systematic review. The keyword search might have missed studies that did not include those keywords. Also, there might be studies not 
available through PubMed search engine that might have been missed in this systematic review. Further, measures developed prior to the last 10 years might still be used in clinical setting and missed in this review. The studies were research studies and may not be reflective of clinical use of these tests. The measures related to sensation were not included in this review and future studies related to hand function items in measures of sensation would likely benefit the clinician.

Numerous limitations in the existing hand function measures were identified in this review. None of the identified measures included performance and self-report in a single measure to give a comprehensive picture of patient's current hand function. There were measures like WMFT and TEMPA that combine instrumented (i.e., Jamar ${ }^{\circledR}$ Dynamometer) and performance assessment of function. There was a lack of studies to provide evidence for the reliability and validity of scores in targeted neurological conditions. The commonly used measures such as the ARAT (Yozbatiran et al., 2008), the FMA(Duncan, Propst, \& Nelson, 1983), SIS-Hand (Duncan et al., 1999) and the MAL (Uswatte, Taub, Morris, Light, \& Thompson, 2006) are developed and validated specific to stroke. The inability to use the measures across other diagnoses is a considerable drawback of the currently available measures. This becomes a major disadvantage in the clinic where a therapist may encounter patients/clients with multiple neurological diagnoses in a day or patients/clients who have dual diagnoses for which these measures lose evidence for validity of scores. There are measures like the DASH (Hudak et al., 1996) that have been developed specifically for people with orthopedic conditions and lack items specific to people with neurological conditions (such as spasticity) reducing its evidence for validity of scores. The currently available measures have focused exclusively on motor assessment of function. There is a lack of measures assessing sensory capacity of the hand with absence of measurement of active haptic mode of hand function. The sensory function of 
the hand is important for recovery and documentation of specific sensory aspects of hand function is critical. The FMA attempts to evaluate sensation however stops with assessment of passive sensory function and ignores the haptic function of hand. Furthermore, some of the measures are lengthy (e.g., FMA) or need multiple pieces of equipment set up (e.g., AMAT), making them unlikely to be utilized in a busy clinic. This review has highlighted the need to develop hand function measures with strong conceptual foundations (Baker et al., 2015), involving people with hand function deficits, and using state-of-the-art measurement techniques. 
Table 2.1. Table of Evidence: Self-report Measures

\begin{tabular}{|c|c|c|c|c|c|c|}
\hline $\begin{array}{l}\text { Measure } \\
\text { Author(s), } \\
\text { year }\end{array}$ & Construct & Dimensions & $\begin{array}{l}\text { Sample item and } \\
\text { response categories }\end{array}$ & $\begin{array}{l}\text { Population } \\
\text { measure } \\
\text { developed for }\end{array}$ & $\begin{array}{l}\text { Neurological } \\
\text { population } \\
\text { measure used in }\end{array}$ & $\begin{array}{l}\text { Training and } \\
\text { administration time }\end{array}$ \\
\hline $\begin{array}{l}\text { Disabilities of } \\
\text { Arm Shoulder } \\
\text { and Hand } \\
\text { (DASH) } \\
\quad \text { Hudak, et } \\
\text { al., } 1996 \text { [1] }\end{array}$ & $\begin{array}{l}\text { Bimanual and } \\
\text { unimanual hand } \\
\text { function and } \\
\text { symptoms in daily } \\
\text { activities, includes } \\
\text { optional } \\
\text { sports/performing } \\
\text { arts or work module }\end{array}$ & $\begin{array}{l}\text { ICF: Body Function, } \\
\text { Activity and } \\
\text { Participation } \\
\text { Hand Function: } \\
\text { Prehension, non- } \\
\text { prehensile, skilled } \\
\text { movements, } \\
\text { transport and tactile } \\
\text { sensing. }\end{array}$ & $\begin{array}{l}\text { Rate ability to do the } \\
\text { following activity in the } \\
\text { past week. "Open a tight } \\
\text { or new jar." } \\
\text { Response: Difficulty } \\
\text { rated as - 1: No, 2: Mild, } \\
\text { 3: Moderate, 4: Severe, } \\
\text { 5: Unable }\end{array}$ & $\begin{array}{l}\text { Musculoskeletal } \\
\text { conditions }\end{array}$ & $\begin{array}{l}\text { Multiple } \\
\text { Sclerosis, Stroke }\end{array}$ & $\begin{array}{l}5 \text { to } 30 \text { minutes to } \\
\text { administer } 38 \text { items with } \\
\text { no training needed, } \\
\text { manual scoring, shorter } \\
\text { version available in } 10- \\
\text { item format }\end{array}$ \\
\hline $\begin{array}{l}\text { ABILHAND } \\
\text { Penta et al., } \\
1998[2]\end{array}$ & $\begin{array}{l}\text { Bimanual ability } \\
\text { across a variety of } \\
\text { motor impairments } \\
\text { specific to diagnosis }\end{array}$ & $\begin{array}{l}\text { ICF: Activity and } \\
\text { Participation } \\
\text { Hand Function: } \\
\text { Prehension }\end{array}$ & $\begin{array}{l}\text { Indicate if you can do the } \\
\text { activity with both hands. } \\
\text { "Hammer a nail." } \\
\text { Response: N/A: Not } \\
\text { applicable/Not an activity } \\
\text { you attempt, 0: Not at all, } \\
\text { 1: Only partially or with } \\
\text { great difficulty or slowly, } \\
\text { 2: Fully and easily }\end{array}$ & $\begin{array}{l}\text { Rheumatoid } \\
\text { arthritis }\end{array}$ & $\begin{array}{l}\text { Stroke, Multiple } \\
\text { Sclerosis, } \\
\text { Parkinson } \\
\text { disease }\end{array}$ & $\begin{array}{l}\geq 10 \text { minutes to } \\
\text { administer } 23 \text { items by } \\
\text { interview with no training } \\
\text { needed and online Rasch } \\
\text { based automated scoring }\end{array}$ \\
\hline $\begin{array}{l}\text { Motor Activity } \\
\text { Log (MAL) } \\
\text { van der Lee } \\
\text { et al., } 1999 \text { [3] }\end{array}$ & $\begin{array}{l}\text { Bimanual and } \\
\text { unimanual activities } \\
\text { of daily living }\end{array}$ & $\begin{array}{l}\text { ICF: Activity and } \\
\text { Participation } \\
\text { (Includes caregiver } \\
\text { report) } \\
\text { Hand Function: } \\
\text { Prehensile, non- } \\
\text { prehensile skilled } \\
\text { movements, and } \\
\text { transport }\end{array}$ & $\begin{array}{l}\text { You will use two } \\
\text { separate rating scales to } \\
\text { describe how much and } \\
\text { how well you use your } \\
\text { weaker arm while you } \\
\text { are doing specific } \\
\text { activities like "Open } \\
\text { Drawer." } \\
\text { Response: From 0: Did } \\
\text { not use weak arm at all, } \\
\text { to 5: Used weak arm as } \\
\text { good as before the stroke }\end{array}$ & $\begin{array}{l}\text { Stroke, } \\
\text { Constraint } \\
\text { induced } \\
\text { movement } \\
\text { therapy trial }\end{array}$ & Stroke & $\begin{array}{l}20 \text { minutes to administer } \\
28 \text { tasks, versions with } \\
45,30,14 \text { tasks are also } \\
\text { available, training with } \\
\text { reading a instruction } \\
\text { manual, manual scoring } \\
\text { additional time }\end{array}$ \\
\hline
\end{tabular}


Table 2.1. Table of Evidence: Self-report Measures (Continued)

\begin{tabular}{|c|c|c|c|c|c|c|}
\hline $\begin{array}{l}\text { Measure } \\
\text { Author(s), year }\end{array}$ & Construct & Dimensions & Sample item and response categories & $\begin{array}{l}\text { Population } \\
\text { measure was } \\
\text { developed }\end{array}$ & $\begin{array}{l}\text { Neurological } \\
\text { population } \\
\text { measure used in }\end{array}$ & $\begin{array}{l}\text { Training and } \\
\text { administration time }\end{array}$ \\
\hline $\begin{array}{l}\text { Stroke Impact Scale } \\
\text { - Hand Domain } 3.0 \\
\text { (SIS-HD) } \\
\text { Duncan et al., } \\
1999 \text { [4] }\end{array}$ & $\begin{array}{l}\text { Bimanual } \\
\text { and } \\
\text { unimanual }\end{array}$ & $\begin{array}{l}\text { ICF: Activity and } \\
\text { Participation } \\
\text { Hand Function: } \\
\text { Prehension and } \\
\text { non-prehensile } \\
\text { skilled } \\
\text { movements }\end{array}$ & $\begin{array}{l}\text { In the past } 2 \text { weeks, how difficult was } \\
\text { it to use your hand that was most } \\
\text { affected by your stroke to... "Turn a } \\
\text { doorknob?" } \\
\text { Response: } 5 \text { : Not difficult at all, 4: A } \\
\text { little difficult, 3: Somewhat difficult, } \\
\text { 2: Very difficult, 1: Could not do at all }\end{array}$ & Stroke & Stroke & $\begin{array}{l}5 \text { minutes to } \\
\text { administer } 5 \text { items, no } \\
\text { training. }\end{array}$ \\
\hline $\begin{array}{l}\text { Manual Ability } \\
\text { Measure-16 } \\
\text { (MAM) } \\
\quad \text { Chen et al., } 2005 \\
\text { [5] }\end{array}$ & $\begin{array}{l}\text { Bimanual } \\
\text { and } \\
\text { unimanual }\end{array}$ & $\begin{array}{l}\text { ICF: Activity and } \\
\text { Participation } \\
\text { Hand Function: } \\
\text { Prehension }\end{array}$ & $\begin{array}{l}\text { Please put a number to indicate how } \\
\text { easy or how hard it is for you to do the } \\
\text { following tasks "Wring a towel." } \\
\text { Response: 4: Easy, 3: A little hard, 2: } \\
\text { Very hard, 1: I can't do, N/A: Not } \\
\text { Applicable }\end{array}$ & $\begin{array}{l}\text { Musculo- } \\
\text { skeletal } \\
\text { conditions }\end{array}$ & $\begin{array}{l}\text { Neurological } \\
\text { conditions } \\
\text { including stroke }\end{array}$ & $\begin{array}{l}\text { Administration time } \\
\text { not reported, has } 16, \\
20,36 \text {-item formats } \\
\text { with manual scoring } \\
\text { and no training } \\
\text { needed }\end{array}$ \\
\hline $\begin{array}{l}\text { Neurological } \\
\text { Quality of Life - } \\
\text { Upper Extremity } \\
\text { v1.0 (NeuroQOL- } \\
\text { UE)) } \\
\text { Carlozzi et al., } \\
2011[6]\end{array}$ & $\begin{array}{l}\text { Bimanual } \\
\text { function of } \\
\text { the upper } \\
\text { extremities } \\
\text { affecting } \\
\text { quality of } \\
\text { life }\end{array}$ & $\begin{array}{l}\text { ICF: Activity and } \\
\text { Participation } \\
\text { Hand Function: } \\
\text { Prehensile, non- } \\
\text { prehensile skilled } \\
\text { movements, and } \\
\text { transport }\end{array}$ & $\begin{array}{l}\text { Please respond to each question or } \\
\text { statement by marking one box per } \\
\text { row. "Are you able to brush your } \\
\text { teeth." Response: 5: Without any } \\
\text { difficulty, 4: With a little difficulty, 3: } \\
\text { With some difficulty, 2: With much } \\
\text { difficulty, 1: Unable to do }\end{array}$ & $\begin{array}{l}\text { Traumatic } \\
\text { Brain Injury }\end{array}$ & $\begin{array}{l}\text { Diverse } \\
\text { population, } \\
\text { Stroke, Multiple } \\
\text { Sclerosis, } \\
\text { Parkinson } \\
\text { Disease }\end{array}$ & $\begin{array}{l}<2 \text { minutes, } \\
\text { Computer adapted } \\
\text { testing format and } \\
\text { paper-based versions } \\
\text { with } 8 \text { to } 10 \text { items } \\
\text { available }\end{array}$ \\
\hline $\begin{array}{l}\text { Patient Reported } \\
\text { Outcome Measure } \\
\text { Information System } \\
\text { - Upper Extremity } \\
\text { v1.2 (PROMIS-UE) } \\
\quad \text { Hung et al., } 2011 \\
\text { [7] }\end{array}$ & Bimanual & $\begin{array}{l}\text { ICF: Activity and } \\
\text { Participation } \\
\text { Hand Function: } \\
\text { Prehensile, non- } \\
\text { prehensile skilled } \\
\text { movements, and } \\
\text { transport }\end{array}$ & $\begin{array}{l}\text { Please respond to each question or } \\
\text { statement by marking one box per } \\
\text { row. "Are you able to reach into a } \\
\text { high cupboard." Response: } 5 \text { : } \\
\text { Without any difficulty, 4: With a little } \\
\text { difficulty, 3: With some difficulty, } 2 \text { : } \\
\text { With much difficulty, 1: Unable to do }\end{array}$ & $\begin{array}{l}\text { Diverse } \\
\text { populations }\end{array}$ & $\begin{array}{l}\text { Neurologic } \\
\text { populations }\end{array}$ & $\begin{array}{l}\text { Administration time } \\
\text { not reported, } 16 \text { items } \\
\text { with online scoring } \\
\text { available }\end{array}$ \\
\hline
\end{tabular}


Table 2.2. Table of Evidence: Self-report Measures Psychometric Properties

\begin{tabular}{|c|c|c|c|}
\hline $\begin{array}{l}\text { Measure } \\
\text { Author(s), } \\
\text { year }\end{array}$ & Evidence for reliability of scores & Evidence for validity of scores & Evidence for responsiveness \\
\hline $\begin{array}{l}\text { Disabilities } \\
\text { of Arm } \\
\text { Shoulder and } \\
\text { Hand } \\
\text { (DASH) } \\
\quad \text { Hudak et } \\
\text { al., } 1996[1]\end{array}$ & $\begin{array}{l}\text { Test-retest: In Athletes, ICC }= \\
0.536 \text { [2]; Proximal Humerus } \\
\text { Fractures, ICC } 2,1=0.928 \\
(0.860 \text { to } 0.963) \text { [3]; Total } \\
\text { elbow arthroplasty, ICC }=0.96 \\
\text { [4]. }\end{array}$ & $\begin{array}{l}\text { Criterion-related evidence for validity of scores: } \\
\text { Neck pain - concurrent evidence for validity of } \\
\text { scores with VAS }=0.55 \text {, NDI }=0.82-0.84 \text { [5] } \\
\text { Construct-related evidence for validity of scores: } \\
\text { Neck pain - convergent validity of scores - SF-12 } \\
\text { physical component }=0.62 \text { [6], Carpometacarpal } \\
\text { arthroplasty - discriminant evidence for validity } \\
\text { of scores -SF } 36 \text { physical component }=-0.49 \text { [7] } \\
\text { Content and Face validity evidence: N/E for } \\
\text { neurological conditions. Poor for stroke [8]. } \\
\text { Established for musculoskeletal conditions [1]. }\end{array}$ & $\begin{array}{l}\text { Shoulder, arm and hand }=1.92[6], \text { Neck pain }=1.38 \\
\text { [6], Total elbow arthroplasty }- \text { Effect size }=0.56, \\
\text { Sensitivity }=0.59 \text {, Specificity }=0.71 \text { [4]. Japanese: } \\
\text { Wrist disorders }- \text { Effect size }=1.20 \text {, Standard } \\
\text { response mean }=1.30[9] \\
\text { MDC: Athletes }-10[10] \text {, Proximal humeral fractures }- \\
16.1 \text { [3] } \\
\text { MCID: Athletes }-10[10] \text {, Post-op surgery for UE }-19 \\
\text { (15 to } 23 \text { ) for patients reporting "much better" [11] } \\
\text { Ceiling effects: Elbow disorders } 1-6 \% \text { [4], Proximal } \\
\text { humeral fractures [3] - 7\% ceiling. Floor effect was } \\
\text { found to } 0 \% \text { in elbow disorders [4] }\end{array}$ \\
\hline $\begin{array}{l}\text { ABILHAND } \\
\quad \text { Penta et } \\
\text { al., } 1998 \\
{[12]}\end{array}$ & $\begin{array}{l}\text { Stroke: Separation Rasch } \\
\text { evidence for reliability of } \\
\text { scores }=0.90 \text { [13]. Stroke: DIF } \\
\text { analysis - uniformity } \\
\text { established [13]. Stroke, MS, } \\
\text { SCI, PD, cerebellar ataxia, } \\
\text { healthy - Cronbach's alpha }= \\
0.99[14]\end{array}$ & $\begin{array}{l}\text { Stroke - All items fit Rasch model, } 23 \text { items } \\
\text { defined manual ability, item distribution }=1.72 \\
\text { to }-2.18 \text { logits [13]. Content-related evidence for } \\
\text { validity of scores established [13]. Factor } \\
\text { analysis - score explained } 84 \% \text { of variance [14] } \\
\text { Criterion-related evidence for validity of scores: } \\
\text { Stroke- Concurrent evidence for validity of } \\
\text { scores with Jamar (r=0.481), BBT (r=0.481) [14] } \\
\text { Construct-related evidence for validity of scores: } \\
\text { Stroke: Known group - Unaffected limb with } \\
\text { Jamar (r }=0.242) \text {, BBT (r }=0.248) \text { [13]. Affected } \\
\text { limb with Jamar r }=0.562, \text { BBT r }=0.598[13] . \\
\text { Differences between tetraparesis, hemiparesis, } \\
\text { other (MS, PD, cerebellar ataxia) \& controls [14] }\end{array}$ & $\begin{array}{l}\text { Average ability of healthy controls }=89(\mathrm{SE}=8) \text { vs } \\
\text { patients with stroke, MS, PD, Cerebellar ataxia, SCI, } \\
\text { PD group }=63(\mathrm{SE}=17)[14], \text { Stroke: Sensitivity }= \\
92 \% \text { and Specificity }=80 \%[14] \\
\text { MDC: } 0.13 \text { logits }[15] \\
\text { Stroke }- \text { Measurement error in center of scale }=0.36 \\
\text { logits }[15]\end{array}$ \\
\hline $\begin{array}{l}\text { Motor } \\
\text { Activity Log } \\
\text { (MAL) } \\
\quad \text { van der } \\
\text { Lee et al., } \\
1999[16]\end{array}$ & $\begin{array}{l}\text { Test-Retest evidence for } \\
\text { reliability of scores: Stroke: } \\
\text { MAL - Amount of use (AOU) } \\
\text { r }=0.70 \text { to } 0.85, \text { MAL }- \\
\text { Quality of movement (QOM) r } \\
=0.61 \text { to } 0.71[17] . \text { Cronbach's } \\
\text { alpha MAL AOU }=0.88, \text { QOM } \\
=0.91[17] .\end{array}$ & $\begin{array}{l}\text { Stroke: Concurrent evidence for validity of scores } \\
\text { MAL } 28 \text { QOM and SIS-HD } r=0.72[18], \text { MAL } \\
28 \text { QOM and accelerometry } r=0.52[17], \text { MAL } \\
28 \text { and ARAT } r=0.63[18]\end{array}$ & $\begin{array}{l}\text { Responsiveness ratio } \mathrm{AOU}=1.9, \mathrm{QOM}=2.0 \text { with } \\
\text { Spearman rho }=0.16 \text { to } 0.22 . \mathrm{MAL} 14 \mathrm{QOM} \\
\text { participant scale responsiveness ratio }>3[17]\end{array}$ \\
\hline
\end{tabular}


Table 2.2. Table of Evidence: Self-report Measures Psychometric Properties (Continued)

\begin{tabular}{|c|c|c|c|}
\hline $\begin{array}{l}\text { Measure } \\
\text { Author(s), year }\end{array}$ & Evidence for reliability of scores & Evidence for validity of scores & Evidence for responsiveness \\
\hline $\begin{array}{l}\text { Stroke Impact Scale } \\
\text { - Hand Domain } 3.0 \\
\text { (SIS-HD) } \\
\text { Duncan et al., } \\
\text { 1999 [19] }\end{array}$ & $\begin{array}{l}\text { Test-Retest evidence for reliability of scores: } \\
\text { SIS Acute stroke - ICC } 0.70 \text { to } 0.92[20] \\
\text { Cronbach's alpha }=0.83 \text { to } 0.90 \text { [20], SIS HD } \\
\text { ICC }=0.95[21] \text { for SIS in Acute stroke }\end{array}$ & $\begin{array}{l}\text { Concurrent evidence for validity of scores of } \\
\text { SIS-HD and Fugl-Meyer Assessment-Upper } \\
\text { extremity, } r=0.81 \text { [19], Criterion-related } \\
\text { evidence for validity of scores SIS- HD rho = } \\
0.51 \text { to } 0.68 \text { [20] } \\
\text { Construct-related evidence for validity of } \\
\text { scores: Convergent evidence for validity of } \\
\text { scores - SIS and Burden of Stroke Scale } \mathrm{r}=- \\
0.83 \text { [22]. Discriminant evidence for validity } \\
\text { of scores for various degrees of stroke severity } \\
\text { [19] }\end{array}$ & $\begin{array}{l}\text { SIS-HD medium evidence for } \\
\text { responsiveness SRM }=0.52[23] . \\
\text { SIS- HD SEM }=9.4[23] \\
\text { MDC: } \text { SIS- HD }=25.9[24] \\
\text { MCID: SIS-HD }=17.8[24] \\
\text { Floor effect } 2 \% \text { in minor stroke, } \\
40.2 \% \text { in moderate stroke, ceiling } \\
\text { effect } 14.6 \% \text { in minor stroke, } 4.9 \% \\
\text { in moderate stroke }[19]\end{array}$ \\
\hline $\begin{array}{l}\text { Manual Ability } \\
\text { Measure-16 (MAM) } \\
\text { Chen et al., } 2005 \\
\text { [25] }\end{array}$ & $\begin{array}{l}\text { Test-Retest, Inter-rater, Intra-rater evidence } \\
\text { for reliability of scores: N/A } \\
\text { Person separation } 0.93 \text {, Item separation } 0.99 \text {. } \\
\text { Variance explained by measure was } 78.4 \% \\
\text { [25] in Rheumatoid Arthritis, Osteoarthritis, } \\
\text { Carpal tunnel syndrome, tenosynovitis, hand } \\
\text { injuries }\end{array}$ & $\begin{array}{l}\text { Criterion-related evidence for validity of scores: } \\
\text { Moderate correlation with LIFEware physical } \\
\text { function } \mathrm{r}=0.51, \mathrm{SF}-12 \text { moderate correlation } \mathrm{r} \\
=0.44 \text { [26] } \\
\text { Construct-related evidence for validity of } \\
\text { scores: Item discrimination greater than } 0.4 \\
\text { [25]. MAM 16: Known group: Rasch person } \\
\text { evidence for reliability of scores }=0.83 \text {. Fit } \\
\text { statistics not greater than } 1.4 \text {. Item evidence } \\
\text { for reliability of scores }=0.98 \\
\text { Content-related evidence for validity of scores: } \\
\text { DIF } 14 \text { items - Single construct, no misfitting } \\
\text { items [25] }\end{array}$ & Not Available \\
\hline $\begin{array}{l}\text { Neurological } \\
\text { Quality of Life - } \\
\text { Upper Extremity } \\
\text { Section v1.0 (Fine } \\
\text { Motor, ADL) } \\
\text { (NeuroQOL-UE) } \\
\text { Carlozzi et al., } \\
2011 \text { [27] }\end{array}$ & $\begin{array}{l}\text { Test-Retest evidence for reliability of scores: } \\
\text { ICC } 0.73 \text { to } 0.94[28,29] \\
\text { Cronbach's alpha } 0.85 \text { to } 0.97 \text { [28] for } \\
\text { NeuroQOL }\end{array}$ & $\begin{array}{l}\text { Criterion-related evidence for validity of scores: } \\
\text { Predictive: Addressed for entire set of items } \\
\text { [29] } \\
\text { Construct-related evidence for validity of } \\
\text { scores: Short forms correlated to full item bank } \\
(\mathrm{r}=0.82 \text { to } 0.96) \text {, Short forms able to } \\
\text { differentiate people with } 0,1-2,3 \text { reported } \\
\text { diagnoses. Face validity evidence: Patient } \\
\text { telephone interviews, focus groups [27]. }\end{array}$ & Not Available \\
\hline
\end{tabular}


Table 2.2. Table of Evidence: Self-report Measures Psychometric Properties (Continued)

\begin{tabular}{|c|c|c|c|}
\hline Author(s), year & $\begin{array}{l}\text { Evidence for reliability of } \\
\text { scores }\end{array}$ & Evidence for validity of scores & Evidence for responsiveness \\
\hline $\begin{array}{l}\text { Patient Reported } \\
\text { Outcome Measure } \\
\text { Information System - } \\
\text { Upper Extremity Section } \\
\text { v1.2 (PROMIS-UE) }\end{array}$ & $\begin{array}{l}\text { Test-Retest evidence for } \\
\text { reliability of scores: } \geq \\
0.80 \text { [32] } \\
\text { Unidimensionality to } \\
\text { physical function } \\
{[31,30] \text { in Diverse }} \\
\text { population }\end{array}$ & 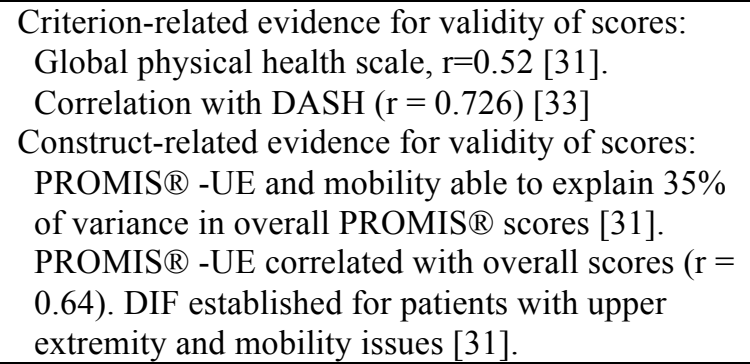 & $\begin{array}{l}\text { Diverse population: Addressed, more responsive } \\
\text { than mobility items [31]. Floor and ceiling } \\
\text { effects: }<1 \% \text {. Diverse population: Skewness and } \\
\text { kurtosis for the estimated upper extremity scores } \\
\text { in the sample were }-1.70(-0.88) \text { and } 2.19 \text { (- } \\
0.07) \text {. } \\
\text { SEM: } 3.3 \text { to } 5.9\end{array}$ \\
\hline \multicolumn{4}{|c|}{ 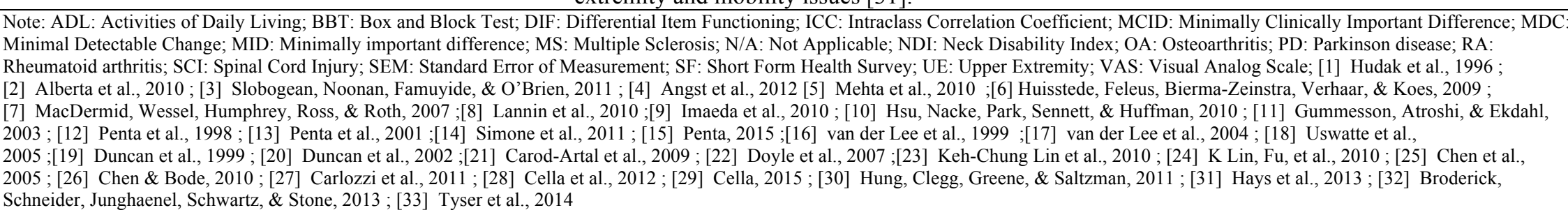 } \\
\hline
\end{tabular}


Table 2.3. Table of Evidence: Performance-based Measures

\begin{tabular}{|c|c|c|c|c|c|c|}
\hline $\begin{array}{l}\text { Measure } \\
\quad \text { Author(s), } \\
\text { year }\end{array}$ & Construct & Dimensions & Sample item and response categories & $\begin{array}{l}\text { Population } \\
\text { measure } \\
\text { developed for }\end{array}$ & $\begin{array}{l}\text { Neurological } \\
\text { population } \\
\text { measure used in }\end{array}$ & $\begin{array}{l}\text { Training and } \\
\text { administration time }\end{array}$ \\
\hline $\begin{array}{l}\text { Jebsen Hand } \\
\text { Function Test } \\
\text { (JHFT) } \\
\text { Jebsen et al., } \\
1969 \text { [1] }\end{array}$ & $\begin{array}{l}\text { Unimanual } \\
\text { activities } \\
\text { of daily } \\
\text { living }\end{array}$ & $\begin{array}{l}\text { ICF: Activity } \\
\text { Hand Function: } \\
\text { Prehension, transport }\end{array}$ & $\begin{array}{l}\text { Checkers: Place your left hand on the } \\
\text { table please. When I say 'Go', use your } \\
\text { left hand to stack these checkers on the } \\
\text { board in front of you as fast as you can } \\
\text { like this one on top of the other } \\
\text { (demonstrate). You may begin with any } \\
\text { checker. Do you understand? Ready? } \\
\text { Go. } \\
\text { Response: Timed }\end{array}$ & Spina Bifida & $\begin{array}{l}\text { Stroke, Brain } \\
\text { injury, Spinal } \\
\text { Cord Injury }\end{array}$ & $\begin{array}{l}15 \text { minutes for } 7 \\
\text { items with } \\
\text { assembling of } \\
\text { materials needed and } \\
\text { training needed but } \\
\text { not specified in the } \\
\text { manual }\end{array}$ \\
\hline $\begin{array}{l}\text { Rivermead } \\
\text { Movement } \\
\text { Assessment - } \\
\text { Upper } \\
\text { Extremity } \\
\text { (RMA-UE) } \\
\quad \text { Lincoln et } \\
\text { al., } 1979[2]\end{array}$ & $\begin{array}{l}\text { Bimanual } \\
\text { and } \\
\text { unimanual } \\
\text { movements } \\
\text { and } \\
\text { activities of } \\
\text { daily living }\end{array}$ & $\begin{array}{l}\text { ICF: Activity } \\
\text { Hand Function: } \\
\text { Prehension, non- } \\
\text { prehensile skilled } \\
\text { movements, } \\
\text { transport }\end{array}$ & $\begin{array}{l}\text { Lying, protract shoulder girdle with arm } \\
\text { in elevation. Arm may be supported. } \\
\text { Response: } 1 \text { if patient can perform } \\
\text { activity, } 0 \text { if he cannot }\end{array}$ & Stroke & Stroke & $\begin{array}{l}45 \text { minutes for total } \\
\text { test and less for } 15 \\
\text { arm items with } \\
\text { assembly of } \\
\text { materials needed }\end{array}$ \\
\hline $\begin{array}{l}\text { Motricity Index } \\
\text { - Upper } \\
\text { Extremity (MI- } \\
\text { UE) } \\
\quad \text { Sunderland } \\
\text { et al., } 1989 \text { [3] }\end{array}$ & $\begin{array}{l}\text { Unimanual } \\
\text { movements }\end{array}$ & $\begin{array}{l}\text { ICF: Body structure } \\
\text { and function } \\
\text { Hand Function: } \\
\text { Prehension, transport }\end{array}$ & $\begin{array}{l}\text { Elbow Flexion to } 90 \text { degrees so that the } \\
\text { arm touches the shoulder. } \\
\text { Response: } 14 \text { points are given if } \\
\text { movement is seen with the elbow out } \\
\text { and the arm horizontal }\end{array}$ & Stroke & Stroke & $\begin{array}{l}\text { Less than } 5 \text { minutes } \\
\text { for } 3 \text { items, only one } \\
\text { cube needed, no } \\
\text { training }\end{array}$ \\
\hline $\begin{array}{l}\text { Action } \\
\text { Research Arm } \\
\text { Test (ARAT) } \\
\quad \text { Lyle, } 1981 \\
{[4]}\end{array}$ & $\begin{array}{l}\text { Unimanual } \\
\text { movements }\end{array}$ & $\begin{array}{l}\text { ICF: Activity } \\
\text { Hand Function: } \\
\text { Prehension, non- } \\
\text { prehensile skilled } \\
\text { movements, } \\
\text { transport }\end{array}$ & $\begin{array}{l}\text { Pick up } 10 \mathrm{~cm} \text { block. } \\
\text { Response: } 0 \text { - No movement, } 3 \text { - } \\
\text { Normal movement }\end{array}$ & Stroke & $\begin{array}{l}\text { Stroke, Multiple } \\
\text { Sclerosis, } \\
\text { Traumatic Brain } \\
\text { Injury }\end{array}$ & $\begin{array}{l}5 \text { to } 20 \text { minutes for } \\
19 \text { movements in } 4 \\
\text { subscales, assembly } \\
\text { of materials needed, } \\
\text { training needed }\end{array}$ \\
\hline
\end{tabular}


Table 2.3. Table of Evidence: Performance-based Measures (Continued)

\begin{tabular}{|c|c|c|c|c|c|c|}
\hline $\begin{array}{l}\text { Measure } \\
\text { Author(s), year }\end{array}$ & Construct & Dimensions & $\begin{array}{l}\text { Sample item and response } \\
\text { categories }\end{array}$ & $\begin{array}{l}\text { Population } \\
\text { measure } \\
\text { developed for }\end{array}$ & $\begin{array}{l}\text { Neurological } \\
\text { population } \\
\text { measure used in }\end{array}$ & $\begin{array}{l}\text { Training and } \\
\text { administration } \\
\text { time }\end{array}$ \\
\hline $\begin{array}{l}\text { Fugl-Meyer } \\
\text { Assessment - Upper } \\
\text { Extremity (FMA- } \\
\text { UE) } \\
\quad \text { Duncan et al., } \\
1983 \text { [5] }\end{array}$ & $\begin{array}{l}\text { Unimanual active and } \\
\text { passive movements, } \\
\text { reflexes, speed, } \\
\text { sensation and joint } \\
\text { pain }\end{array}$ & $\begin{array}{l}\text { ICF: Body structure and } \\
\text { function } \\
\text { Hand Function: } \\
\text { Prehension, non- } \\
\text { prehensile, skilled } \\
\text { movements, tactile } \\
\text { sensing }\end{array}$ & $\begin{array}{l}\text { Pronation-supination, elbow } \\
\text { at } 90 \text { degrees and shoulder } \\
\text { at } 0 \text { degrees } \\
\text { Response: } 0=\text { cannot } \\
\text { perform, } 1=\text { performs } \\
\text { partially and } 2=\text { performs } \\
\text { fully } \\
\text { Response options are } \\
\text { tailored to the item }\end{array}$ & Stroke & Stroke & $\begin{array}{l}20 \text { minutes for } \\
7 \text { sections on } \\
\text { upper } \\
\text { extremity. }\end{array}$ \\
\hline $\begin{array}{l}\text { Functional Test for } \\
\text { the Hemiplegic } \\
\text { Upper Extremity } \\
\text { (FT) } \\
\text { Wilson et al., } \\
\text { 1984 [6] }\end{array}$ & $\begin{array}{l}\text { Bimanual and } \\
\text { unimanual activities } \\
\text { of daily living, } \\
\text { associated reactions } \\
\text { and movements }\end{array}$ & $\begin{array}{l}\text { ICF: Body structure and } \\
\text { function, activity } \\
\text { Hand Function: } \\
\text { Prehension, non- } \\
\text { prehensile skilled } \\
\text { movements, transport }\end{array}$ & $\begin{array}{l}\text { Level 2B. Hand into lap. } \\
\text { Response: Pass or fail based } \\
\text { on ability to complete task } \\
\text { within } 3 \text { minutes except } \\
\text { Level } 1 \text { and } 2 \mathrm{~A} \text {. }\end{array}$ & Stroke & Stroke & $\begin{array}{l}30 \text { minutes for } \\
17 \text { graded } \\
\text { activities }\end{array}$ \\
\hline $\begin{array}{l}\text { Box and Blocks Test } \\
\text { Mathiowetz et } \\
\text { al., } 1985 \text { [7] }\end{array}$ & Unimanual pinch & $\begin{array}{l}\text { ICF: Activity } \\
\text { Hand Function: } \\
\text { Prehension }\end{array}$ & $\begin{array}{l}\text { Number of blocks moved } \\
\text { from one partition to the } \\
\text { other in } 1 \text { minute }\end{array}$ & $\begin{array}{l}\text { Musculo- } \\
\text { skeletal } \\
\text { conditions }\end{array}$ & $\begin{array}{l}\text { Multiple Sclerosis, } \\
\text { Stroke, TBI, } \\
\text { Neuromuscular } \\
\text { diseases, Spinal } \\
\text { Cord Injury } \\
\end{array}$ & $\begin{array}{l}1 \text { minute, one } \\
\text { task }\end{array}$ \\
\hline $\begin{array}{l}\text { Motor Assessment } \\
\text { Scale - Upper } \\
\text { extremity (MAS- } \\
\text { UE) } \\
\quad \text { Carr et al., } 1985 \\
{[8]}\end{array}$ & $\begin{array}{l}\text { Unimanual activities } \\
\text { of daily living and } \\
\text { movements }\end{array}$ & $\begin{array}{l}\text { ICF: Body structure and } \\
\text { function, activity } \\
\text { Hand Function: } \\
\text { Prehension, non- } \\
\text { prehensile skilled } \\
\text { movements, transport }\end{array}$ & $\begin{array}{l}\text { Upper arm, hand and } \\
\text { advanced hand activities } \\
\text { with graded levels from } 0 \\
\text { to } 6 \text { with different tasks } \\
\text { within each grade level. } \\
\text { Advanced hand activities } \\
\text { grade } 6 \text { : Hold a comb and } \\
\text { comb hair at back of head } \\
\text { (shoulder externally } \\
\text { rotated, abducted at } 90 \text { and } \\
\text { head erect). }\end{array}$ & Stroke & Stroke & $\begin{array}{l}15 \text { minutes for } \\
8 \text { activities of } \\
\text { entire measure, } \\
\text { time needed } \\
\text { for materials } \\
\text { and scoring }\end{array}$ \\
\hline
\end{tabular}


Table 2.3. Table of Evidence: Performance-based Measures (Continued)

\begin{tabular}{|c|c|c|c|c|c|c|}
\hline $\begin{array}{l}\text { Measure } \\
\text { Author(s), } \\
\text { year }\end{array}$ & Construct & Dimensions & Sample item and response categories & $\begin{array}{l}\text { Population } \\
\text { measure } \\
\text { developed for }\end{array}$ & $\begin{array}{l}\text { Neurological } \\
\text { population } \\
\text { measure used in }\end{array}$ & $\begin{array}{l}\text { Training and } \\
\text { administration } \\
\text { time }\end{array}$ \\
\hline $\begin{array}{l}\text { Nine Hole Peg } \\
\text { Test (NHPT) } \\
\text { Heller et al., } \\
1987 \text { [9] }\end{array}$ & $\begin{array}{l}\text { Unimanual } \\
\text { pinch }\end{array}$ & $\begin{array}{l}\text { ICF: Activity } \\
\text { Hand } \\
\text { Function: } \\
\text { Prehension }\end{array}$ & Seconds to completion of pegs placed on board & $\begin{array}{l}\text { Musculoskeletal } \\
\text { conditions }\end{array}$ & $\begin{array}{l}\text { Brain Injury, } \\
\text { Stroke, } \\
\text { Parkinson } \\
\text { disease }\end{array}$ & $\begin{array}{l}\text { Varies with } \\
\text { patient's ability, } \\
\text { approximately } 10 \\
\text { minutes }\end{array}$ \\
\hline $\begin{array}{l}\text { Sollerman Hand } \\
\text { Function Test } \\
\text { (SHFT) } \\
\quad \text { Sollerman et } \\
\text { al., } 1995[10]\end{array}$ & $\begin{array}{l}\text { Bimanual } \\
\text { and } \\
\text { unimanual } \\
8 \text { grip } \\
\text { related } \\
\text { tasks }\end{array}$ & $\begin{array}{l}\text { ICF: Activity } \\
\text { Hand } \\
\text { Function: } \\
\text { Prehension, } \\
\text { transport }\end{array}$ & $\begin{array}{l}\text { Fold paper, put into envelope. } \\
\text { Response: 5-point rating scale from } 0 \text { - Task } \\
\text { cannot be performed at all, to } 4 \text { - Task completed } \\
\text { without any difficulty within } 20 \text { seconds and with } \\
\text { the prescribed hand-grip of normal quality. } \\
\text { Subjective estimate of hand function on a } 10 \mathrm{~cm} \\
\text { line from no hand function to full hand function }\end{array}$ & $\begin{array}{l}\text { Musculoskeletal } \\
\text { conditions, } \\
\text { tetraplegia }\end{array}$ & $\begin{array}{l}\text { Tetraplegia, } \\
\text { Stroke }\end{array}$ & $\begin{array}{l}20 \text { minutes for } 20 \\
\text { subtests }\end{array}$ \\
\hline $\begin{array}{l}\text { Test Evaluant la } \\
\text { Performance } \\
\text { des Membres } \\
\text { supérieurs des } \\
\text { Personnes âgées } \\
\text { (TEMPA) }\end{array}$ & $\begin{array}{l}\text { Bimanual } \\
\text { and } \\
\text { unimanual } \\
\text { activities of } \\
\text { daily living }\end{array}$ & $\begin{array}{l}\text { ICF: Activity } \\
\text { Hand } \\
\text { Function: } \\
\text { Prehension, } \\
\text { transport }\end{array}$ & $\begin{array}{l}\text { Open a jar and take a spoonful of coffee. } \\
\text { Grip strength with dynamometer. } \\
\text { Response: Speed, task analysis and function rating. } \\
\text { Task analysis - range, strength, control, } \\
\text { prehension, fine movement. Function rating on a } \\
\text { 4-point ordinal scale }\end{array}$ & $\begin{array}{l}\text { Geriatrics ( } 60 \\
\text { years and over) }\end{array}$ & $\begin{array}{l}\text { Multiple } \\
\text { Sclerosis, } \\
\text { Stroke }\end{array}$ & $\begin{array}{l}9 \text { tasks, demands } \\
\text { on administration } \\
\text { and scoring } \\
\text { unknown. }\end{array}$ \\
\hline \multicolumn{7}{|l|}{$\begin{array}{l}\text { Desrosiers et } \\
\text { al, } 1995 \text { [11] }\end{array}$} \\
\hline $\begin{array}{l}\text { Arm Motor } \\
\text { Ability Test } \\
\text { (AMAT) } \\
\text { Kopp et al., } \\
1997 \text { [12] }\end{array}$ & $\begin{array}{l}\text { Bimanual } \\
\text { and } \\
\text { unimanual } \\
\text { activities of } \\
\text { daily living }\end{array}$ & $\begin{array}{l}\text { ICF: Activity } \\
\text { Hand } \\
\text { Function: } \\
\text { Prehension, } \\
\text { non- } \\
\text { prehensile } \\
\text { skilled } \\
\text { movements, } \\
\text { transport }\end{array}$ & $\begin{array}{l}\text { Cut meat } \\
\text { Response: Functional rating and quality of } \\
\text { movement use a 6-point ordinal scale from } 0 \text { to } 5 \text {. } \\
\text { Performance emphasized not speed. Combined } \\
\text { function and quality rating into one scale is also } \\
\text { available. }\end{array}$ & $\begin{array}{l}\text { Stroke, } \\
\text { Constraint } \\
\text { Induced } \\
\text { Movement } \\
\text { Therapy Trials }\end{array}$ & Stroke & $\begin{array}{l}30 \text { to } 40 \text { minutes, } \\
\text { materials to } \\
\text { assemble and } \\
\text { lengthy to } \\
\text { complete the } 28 \\
\text { tasks with } 1 \text { to } 2 \\
\text { minutes limit per } \\
\text { task. } 9 \text { or } 10 \text { item } \\
\text { version may be } \\
\text { available. }\end{array}$ \\
\hline
\end{tabular}


Table 2.3. Table of Evidence: Performance-based Measures (Continued)

\begin{tabular}{|c|c|c|c|c|c|c|}
\hline $\begin{array}{l}\text { Measure } \\
\text { Author(s), year }\end{array}$ & Construct & Dimensions & Sample item and response categories & $\begin{array}{l}\text { Population } \\
\text { measure } \\
\text { developed for }\end{array}$ & $\begin{array}{l}\text { Neurological } \\
\text { population } \\
\text { measure used } \\
\text { in }\end{array}$ & $\begin{array}{l}\text { Training and } \\
\text { administration time }\end{array}$ \\
\hline $\begin{array}{l}\text { Stroke } \\
\text { Rehabilitation } \\
\text { Assessment of } \\
\text { Movement Scale } \\
\text { (STREAM) } \\
\quad \text { Daley et al., } \\
\text { 1999 [13] }\end{array}$ & $\begin{array}{l}\text { Unimanual } \\
\text { movements }\end{array}$ & $\begin{array}{l}\text { ICF: Body } \\
\text { structure and } \\
\text { function } \\
\text { Hand Function: } \\
\text { Non-prehensile } \\
\text { skilled } \\
\text { movements, } \\
\text { transport }\end{array}$ & $\begin{array}{l}\text { Shrug your shoulders as high as you can. } \\
\text { Response: } 4 \text { point ordinal scale, } 0 \text { - unable } \\
\text { to perform the test activity through any } \\
\text { appreciable range, } 3 \text { - able to complete } \\
\text { the activity independently with a greatly } \\
\text { normal movement pattern without an aid }\end{array}$ & Stroke & Stroke & $\begin{array}{l}20 \text { minutes entire test, } \\
10 \text { upper limb items } \\
\text { with no prehensile } \\
\text { items }\end{array}$ \\
\hline $\begin{array}{l}\text { Wolf Motor } \\
\text { Function Test } \\
\text { (WMFT) } \\
\text { Wolf et al., } \\
2001[14]\end{array}$ & $\begin{array}{l}\text { Bimanual and } \\
\text { unimanual } \\
\text { activities of } \\
\text { daily living }\end{array}$ & $\begin{array}{l}\text { ICF: Activity } \\
\text { Hand Function: } \\
\text { Prehension, } \\
\text { non-prehensile } \\
\text { skilled } \\
\text { movements, } \\
\text { transport }\end{array}$ & $\begin{array}{l}\text { Place your forearm on the table as quickly } \\
\text { as you can. Do it just like this (examiner } \\
\text { demonstrates). At the end of the } \\
\text { movement, your forearm and hand } \\
\text { should be touching the surface of the } \\
\text { table. Do this as quickly as you can. } \\
\text { (repeat instructions) Do you have any } \\
\text { questions? Ready, set, go. } \\
\text { Response: Time score for speed and } \\
\text { Functional ability scale is a 6-point } \\
\text { ordinal scale where } 0 \text {-does not attempt } \\
\text { with the involved arm and } 5 \text { - arm does } \\
\text { participate/movement appears to be } \\
\text { normal }\end{array}$ & $\begin{array}{l}\text { Stroke, } \\
\text { Constraint } \\
\text { Induced } \\
\text { Movement } \\
\text { Therapy } \\
\text { Trials }\end{array}$ & $\begin{array}{l}\text { Stroke, } \\
\text { Traumatic } \\
\text { Brain Injury }\end{array}$ & $\begin{array}{l}60 \text { minutes for each } \\
\text { upper extremity tested } \\
\text { on } 15 \text { items. } \\
\text { Materials, training } \\
\text { with a DVD and } \\
\text { scoring time needed. }\end{array}$ \\
\hline $\begin{array}{l}\text { Chedokee Arm } \\
\text { and Hand } \\
\text { Inventory } \\
\text { (CAHAI) } \\
\text { Barreca et al., } \\
2004 \text { [15] }\end{array}$ & $\begin{array}{l}\text { Bimanual } \\
\text { activities of } \\
\text { daily living }\end{array}$ & $\begin{array}{l}\text { ICF: Activity } \\
\text { Hand Function: } \\
\text { Prehension, } \\
\text { non-prehensile } \\
\text { skilled } \\
\text { movements, } \\
\text { transport }\end{array}$ & $\begin{array}{l}\text { Draw a straight line the length of the ruler } \\
\text { using both of your hands } \\
\text { Response: } 7 \text { point activity scale from } 1 \text { - } \\
\text { Total assistance to } 7 \text { - Complete } \\
\text { independence }\end{array}$ & Stroke & $\begin{array}{l}\text { Stroke, Brain } \\
\text { injury }\end{array}$ & $\begin{array}{l}30 \text { minutes } \\
\text { Time for materials, } \\
\text { training and scoring } \\
\text { needed. }\end{array}$ \\
\hline
\end{tabular}


Table 2.4. Table of Evidence: Performance-based Measures Psychometric Properties

\begin{tabular}{|c|c|c|c|}
\hline $\begin{array}{l}\text { Measure } \\
\quad \text { Author(s), year }\end{array}$ & Evidence for reliability of scores & Evidence for validity of scores & $\begin{array}{l}\text { Evidence for } \\
\text { responsiveness }\end{array}$ \\
\hline $\begin{array}{l}\text { Jebsen Hand } \\
\text { Function Test } \\
\text { (JHFT) } \\
\text { Jebsen et al., } \\
1969 \text { [1] }\end{array}$ & $\begin{array}{l}\text { Test-retest evidence for reliability of scores: } \\
\text { Stroke: } r=0.92 \text { [2], Tetraplegia: Dominant } \\
\text { hand } r=0.91-0.99 \text { [1] dominant hand } \\
\text { 'writing' } r=0.67 \text {, non-dominant hand } r=0.78 \\
-0.92, \text { non-dominant hand 'feeding' } r=0.60 \\
\text { and non-dominant hand 'large objects' } r= \\
0.67 . \text { No practice effect was observed [1]. } \\
\text { Inter-rater evidence for reliability of scores: } \\
\text { Stroke: Portuguese version - ICC }=1.0 \text { [3] } \\
\text { Healthy ICC=0.82 -1.00 [4] } \\
\text { Intra-rater evidence for reliability of scores: } \\
\text { Stroke: Portuguese version - ICC }=0.997 \text { [3] } \\
\text { Cronbach's alpha = 0.924. Stroke: Portuguese } \\
\text { version of JHFT 'writing' alpha }=0.884 \text {, } \\
\text { Pearson's } r \text { (item to total score) for 'small } \\
\text { objects' } r=0.657 \text { [3] }\end{array}$ & $\begin{array}{l}\text { Criterion-related evidence for validity of scores: } \\
\text { Concurrent evidence for validity of scores: Stroke: } \\
\text { Correlation with grip strength } \mathrm{r}=0.79-0.81 \text {, pinch } \\
\text { strength } \mathrm{r}=0.60=0.79, \text { ARAT } \mathrm{r}=0.87-0.95 \text {, NHPT r } \\
0.84-0.97, \text { SIS-HD r } 0.61-0.83 \text { [2] Correlation with } \\
\text { MHQ subtests for RA, OA, Fracture } \mathrm{r}=0.10-0.41[5] . \\
\text { CTS correlation for ADL } \mathrm{r}=0.68 \text {, function } \mathrm{r}=0.69[5] . \\
\text { Construct-related evidence for validity of scores: } \\
\text { Discriminant evidence for validity of scores Positive }= \\
\text { change in MHQ scores }>20, \text { negative }=\text { change in MHQ } \\
\text { score } \leq 20 . \text { ROC area under curve for RA }=0.52, \mathrm{OA}= \\
0.58, \mathrm{CTS}=0.66, \text { Fracture }=0.59 .\end{array}$ & $\begin{array}{l}\text { Stroke: } 1-3 \text { months post } \\
\text { stroke, effect size }=0.69 \\
\text { and between } 1-6 \text { months } \\
\text { post stroke }=0.73[2] \\
\text { Effect size, CTS }=0.05, \\
\text { Fracture }=0.35, \mathrm{RA}= \\
0.47, \mathrm{OA}=0.67[5]\end{array}$ \\
\hline $\begin{array}{l}\text { Rivermead } \\
\text { Movement } \\
\text { Assessment - } \\
\text { Upper Extremity } \\
\text { (RMA-UE) } \\
\quad \text { Lincoln et al., } \\
1979[6]\end{array}$ & $\begin{array}{l}\text { Stroke: Test-Retest Evidence for reliability of } \\
\text { scores: RMA-UE r }=0.88 \text { [6]. } \\
\text { Inter-rater: Significant difference in scoring } \\
\text { across rater as compared to variability between } \\
\text { patients. Revised scoring produced for RMA- } \\
\text { UE - testing of revised version not done [6]. } \\
\text { Stroke: RMA-UE Cronbach's alpha }=0.95 \text {, } \\
\text { ICC }=0.93 \text { [7] }\end{array}$ & $\begin{array}{l}\text { Content-related evidence for validity of scores: } \\
\text { Unidimensionality with Guttman scale [ } 6] \text {. } \\
\text { Criterion-related evidence for validity of scores: RMA } \\
\text { concurrent with Barthel Index across each assessment } \\
\text { period initial }(\mathrm{r}=0.84), 1 \text { month }(\mathrm{r}=0.78) \text {, and one year } \\
(\mathrm{r}=0.63) \text { [8]. } \\
\text { Predictive evidence for validity of scores }- \text { low RMA } \\
\text { scores at } 6 \text { weeks post stroke predicted with poor } \\
\text { prognosis to ambulate [9]. } \\
\text { Construct-related evidence for validity of scores: } \\
\text { Convergent evidence for validity of scores RMA-UE } \\
\text { with MI } \mathrm{r}=0.74 \text { [9], RMA with FIM cognition } \mathrm{r}=0.52 \\
\text { [10], RMA-UE with FIM } \mathrm{r}=0.386-0.483 \text { [7]. } \\
\text { Alternative forms convergent evidence for validity of } \\
\text { scores with verbal and performance methods of } \\
\text { completion } \mathrm{r}=0.98 \text { [11], Known group validity of scores } \\
\text { with RMA score and infarct size at initial } \mathrm{r}=-0.52,1 \\
\text { month } \mathrm{r}=-0.47 \text {, and } 1 \text { year } \mathrm{r}=-0.53 \text { [8]. }\end{array}$ & $\begin{array}{l}\text { All } 3 \text { sections are sensitive } \\
\text { to change, arm section } \\
\text { least responsive when } \\
\text { compared to FIM. RMA } \\
\text { ES }=0.38, \text { SRM }=0.60 \text { [7] } \\
\text { MCID: } \pm 3 \text { points likely } \\
\text { to represent a clinically } \\
\text { relevant change in } \\
\text { functional level [12]. } \\
\text { Large ceiling on RMA } \\
\text { gross motor subscale } \\
\text { when compared to } \\
\text { HIMAT [13]. Floor and } \\
\text { ceiling not established for } \\
\text { RMA-UE. }\end{array}$ \\
\hline
\end{tabular}


Table 2.4. Table of Evidence: Performance-based Measures Psychometric Properties (Continued)

\begin{tabular}{|c|c|c|c|}
\hline $\begin{array}{l}\text { Measure } \\
\text { Author(s), year }\end{array}$ & $\begin{array}{l}\text { Evidence for reliability of } \\
\text { scores }\end{array}$ & Evidence for validity of scores & Evidence for responsiveness \\
\hline $\begin{array}{l}\text { Motricity Index - Upper } \\
\text { Extremity (MI-UE) } \\
\text { Sunderland et al., } 1989 \\
{[14]}\end{array}$ & $\begin{array}{l}\text { Stroke: Inter-rater } \\
\text { evidence for reliability } \\
\text { of scores: MI-UE }=0.88 \\
\text { (Spearman's rho) [15]. } \\
\text { Stroke: Cronabach's } \\
\text { alpha }=0.968[15] .\end{array}$ & $\begin{array}{l}\text { Stroke: Criterion-related evidence for validity of } \\
\text { scores: Concurrent with dynamometry of UE r }= \\
0.89 \text { [16]. RMA-UE with MI Spearman's rho } 6 \\
\text { weeks }=0.76,12 \text { weeks }=0.73,18 \text { weeks }=0.74 \text {. } \\
\text { Predictive for acute stroke at } 1,3,6 \text { months post } \\
\text { stroke [15]. } \\
\text { Predictive MI combined with trunk control test at } \\
6 \text { weeks predicted failure to walk by } 18 \text { weeks } \\
\text { [15]. }\end{array}$ & $\begin{array}{l}\text { Response mean }=0.96, \text { Effect size }=0.27[17] . \\
\text { Stroke: Floor/Ceiling effects: } 57 \% \text { had had } \\
\text { measureable pinch grip within first } 3 \text { weeks of } \\
\text { a stroke, } 2 \% \text { had normal pinch grip [14]. } \\
\text { Sensitivity: Early change with acute stroke can } \\
\text { be detected [14]. }\end{array}$ \\
\hline $\begin{array}{l}\text { Action Research Arm Test } \\
\text { (ARAT) } \\
\quad \text { Lyle, } 1981[18]\end{array}$ & $\begin{array}{l}\text { Test-retest evidence for } \\
\text { reliability of scores: } \\
\text { ICC }=0.894 \text { to } 0.976, \\
\text { rho }=0.897 \text { to } 0.976 \\
\text { [19], r }=0.98[18] \\
\text { Inter-rater evidence for } \\
\text { reliability of scores: } \\
\text { Acute stroke: ICC }= \\
0.92 \text { [20], Chronic } \\
\text { stroke: ICC = 0.995 } \\
\text { [21]. Chronic and acute } \\
\text { stroke, MS, TBI: ICC }= \\
0.998 \text { [22], Chronic } \\
\text { stroke: Inter-rater: ICC } \\
=0.9986, \text { rho }=0.96 \\
\text { [23] } \\
\text { Intra-rater evidence for } \\
\text { reliability of scores: } \\
\text { Chronic stroke: ICC }= \\
\text { 0.989 [21] }\end{array}$ & $\begin{array}{l}\text { Criterion-related evidence for validity of scores: } \\
\text { Chronic stroke: Concurrent with FMA-UE [21] r = } \\
0.94[23] \text { and MAS }[21] \text {. } \\
\text { Construct-related evidence for validity of scores: } \\
\text { Convergent with FMA motor domain }(\text { rho }= \\
0.925), \text { MI }(\text { rho }=0.811) \text { and BBT }(\text { rho }=0.951) \\
{[19]}\end{array}$ & $\begin{array}{l}\text { Chronic Stroke: Responsiveness ratio }=2.03,1.7 \\
\text { points with summing items [21]. } \\
\text { Acute stroke: Effect size between } 14 \text { and } 180 \\
\text { days }=0.79[24] \\
\text { Effect size at } 1 \text { and } 3 \text { months post stroke }=0.55 \text {, } \\
1 \text { and } 6 \text { months post stroke }=0.63 \text { [25], } 0 \text { and } 90 \\
\text { days post stroke } 1.390 \text { [26]. MID, MDC: N/E } \\
\text { Chronic stroke: MCID = } 10 \% \text { of total }(5.7 \\
\text { points), } \\
\text { Acute stroke: MCID Dominant side }=12 \text { points } \\
(E S=0.78), \text { Non-dominant side }=17 \text { points (ES } \\
=1.10) \text { [21]. } \\
\text { Normative Data: Established [21,25] } \\
\text { Acute stroke: Floor effects for raw scores }<3 \\
\text { and ceiling for }>54[20], \text { Floor effects } 14 \text { days } \\
\text { post stroke }=41.5 \%[24], \text { ceiling effects } 180 \\
\text { days post stroke }=22.6 \% \text { [24]. Acute stroke: } \\
\text { Cronbach's alpha } 0.985 \text { [20]. }\end{array}$ \\
\hline
\end{tabular}


Table 2.4. Table of Evidence: Performance-based Measures Psychometric Properties (Continued)

\begin{tabular}{|c|c|c|c|}
\hline $\begin{array}{l}\text { Measure } \\
\text { Author(s), year }\end{array}$ & $\begin{array}{l}\text { Evidence for } \\
\text { reliability of scores }\end{array}$ & Evidence for validity of scores & Evidence for responsiveness \\
\hline $\begin{array}{l}\text { Fugl-Meyer } \\
\text { Assessment - } \\
\text { Upper Extremity } \\
\text { (FMA-UE) }\end{array}$ & $\begin{array}{l}\text { Stroke, MS, TBI: Test- } \\
\text { Retest Evidence for } \\
\text { reliability of scores: } \\
\text { FMA Total ICC }=0.97 \\
{[28],}\end{array}$ & $\begin{array}{l}\text { Criterion-related evidence for validity of scores: } \\
\text { Stroke: Concurrent evidence for validity of scores: FMA and MAS r }=0.96 \\
\text { [31], Poor FMA and MAS sitting balance } r=-0.10 \text { [31], } \\
\text { Predictive evidence for validity of scores: Motor and sensory FMA scores } 5 \\
\text { days post stroke predictive of motor recovery } 6 \text { months post-stroke [32]. }\end{array}$ & $\begin{array}{l}\text { Chronic Stroke: Responsiveness } \\
\text { ratio }=0.41[41] \\
\text { FMA }- \text { Sensation subscale } \\
\text { SRM at } 14-180 \text { days }=0.67 \\
{[29] .}\end{array}$ \\
\hline $\begin{array}{l}\text { Duncan et al., } \\
1983 \text { [27] }\end{array}$ & $\begin{array}{l}\text { Chronic stroke: Inter- } \\
\text { rater evidence for } \\
\text { reliability of scores: } \\
\text { FMA total r }=0.98 \\
\text { to } 0.99, \text { FMA-UE r } \\
=0.98 \text { to } 0.995 \text { [27]. } \\
\text { Stroke: Cronbach's } \\
\text { alpha }=0.94 \text { to } 0.98 \\
\text { across } 4 \\
\text { administrations [29]. } \\
\text { FMA-UE to FMA r } \\
=0.97 \text { [30] }\end{array}$ & $\begin{array}{l}\text { Construct-related evidence for validity of scores: } \\
\text { Acute stroke: Convergent with FMA and Barthel Index }(r=0.86-0.89)[33] \\
\text { and FIM }(r=0.63)[34] . \text { Known group evidence for validity of scores: FMA } \\
\text { distinguished between dependent, partly dependent and independent levels of } \\
\text { self-care [35]. } \\
\text { Chronic Stroke: Convergent with FMA-UE and Barthel Index }(r=0.75)[36] \text {, } \\
\text { FMA and ARAT (rho =0.73) [37], WMFT time }(\text { rho }=0.76) \text { [37], FIM-motor } \\
(\text { rho }=0.49) \text { [37]. } \\
\text { Content-related evidence for validity of scores: } \\
\text { Acute stroke: FMA-UE modified with } 3 \text { reflex items removed, adequate fit } \\
\text { using Rasch Analysis except for hook grasp item at admission and } 6 \text { months } \\
\text { post stroke [38]. Chronic stroke: Unidimensional items within the FMA-UE } \\
\text { [39]. } \\
\text { Face validity evidence: Established and heavily weighted for UE [40]. }\end{array}$ & $\begin{array}{l}\text { Shortened FMA ES }=0.53, \\
\text { FMA ES }=0.045 \text { [42] FMA- } \\
\text { UE: } \mathrm{MDC}=5.2 \text { points [43], } \\
\text { MCID = } 10 \text { points }=1.5 \text { change } \\
\text { in discharge FIM [44]. } \\
\text { Floor/Ceiling effects } \\
\text { Stroke: Possible ceiling effects } \\
\text { with hand [39] } \\
\text { Stroke: Cronbach's alpha }= \\
0.94 \text { to } 0.98 \text { across } 4 \\
\text { administrations [29]. FMA-UE } \\
\text { to FMA r }=0.97 \text { [30] }\end{array}$ \\
\hline $\begin{array}{l}\text { Functional Test } \\
\text { for the } \\
\text { Hemiplegic } \\
\text { Upper Extremity } \\
\text { (FT) }\end{array}$ & $\begin{array}{l}\text { Stroke: Inter-rater } \\
\text { evidence for } \\
\text { reliability of scores, } \\
\text { rho }=0.976[45] . \\
\text { Hong Kong version, } \\
\text { rho }=0.93[46] .\end{array}$ & $\begin{array}{l}\text { Criterion-related evidence for validity of scores: Concurrent with FMA r }=0.88 \\
\text { and FMA-UE subscores } r=0.88 \text {. Correlation with FIM self care, } r=0.46 \text { [45] } \\
\text { Construct-related evidence for validity of scores: FT scores accounted for } 87 \% \\
\text { of variance in six other test scores [45] }\end{array}$ & Not available \\
\hline $\begin{array}{l}\text { Wilson et al., } \\
1984 \text { [45] }\end{array}$ & & & \\
\hline
\end{tabular}


Table 2.4. Table of Evidence: Performance-based Measures Psychometric Properties (Continued)

\begin{tabular}{|c|c|c|c|}
\hline $\begin{array}{l}\text { Measure } \\
\text { Author(s), year }\end{array}$ & Evidence for reliability of scores & Evidence for validity of scores & Evidence for responsiveness \\
\hline $\begin{array}{l}\text { Box and Blocks } \\
\text { Test (BBT) } \\
\quad \text { Mathiowetz et } \\
\text { al., } 1985 \text { [47] }\end{array}$ & $\begin{array}{l}\text { Acute and Chronic Stroke: Test- } \\
\text { retest reliability: More affected } \\
\text { hand } r=0.98, \text { Less affected } \\
\text { hand } r=0.93 \text { [48]. Upper limb } \\
\text { impairment: Right hand: ICC }= \\
\text { 0.90, Left hand: ICC }=0.89 \text { [49]. } \\
\text { Stroke, MS, TBI: ICC }=0.96, \\
\text { Spastic Hemiplegia: ICC }=0.95 \\
\text { [50] } \\
\text { Inter-rater reliability: Healthy: } \\
\text { Left hand } r=0.99, \text { Right hand } \\
=1.00 \text { [47], Healthy: ICC }= \\
\text { 0.80 [51], Stroke, MS, TBI: } \\
\text { ICC 0.99 [52], Spastic } \\
\text { hemiplegia: } r=0.95 \text { [50], } \\
\text { Fibromyalgia ICC }=0.85 \text { [51]. }\end{array}$ & $\begin{array}{l}\text { Stroke: Criterion-related evidence for validity } \\
\text { of scores: Concurrent post treatment with } \\
\text { NHPT }(\mathrm{r}=-0.71) \text {, ARAT }(\mathrm{r}=0.64) \text {, FMA }(\mathrm{r}= \\
0.35) \text {, SIS }(\mathrm{r}=0.52) \text {, MAL-AOU }(\mathrm{r}=0.49) \text {, } \\
\text { MAL-QOM }(\mathrm{r}=0.52) \text {. Adequate with FMA, } \\
\text { MAL and SIS [53]. Predictive evidence for } \\
\text { validity of scores: At } 1 \text { and } 5 \text { weeks post- } \\
\text { stroke, BBT was best predictor of upper limb } \\
\text { function [54]. } \\
\text { Construct-related evidence for validity of } \\
\text { scores: UE Paresis: Convergent with ARAT ( } \\
=0.95), \text { FMA }(r=0.92) \text {, Hemispheric stroke } \\
\text { scale ( } \mathrm{r}=-0.67), \text { passive joint motion/joint } \\
\text { pain subscale }(\mathrm{r}=0.43), \text { Modified Barthel } \\
\text { Index ( } \mathrm{r}=0.04) \text { [52]. Brain injury associated } \\
\text { paresis (stroke, anoxic brain damage, TBI, } \\
\text { SCI): Convergent with resistance to passive } \\
\text { movement }(\mathrm{r}=-0.680) \text { [52]. }\end{array}$ & $\begin{array}{l}\text { Acute Stroke: } 1 \text { and } 4 \text { weeks post-stroke SRM: } 0.8 \\
\text { [54], } 0 \text { and } 3 \text { weeks SRM }=0.74 \text { [53]. MDC: Acute } \\
\text { and Chronic stroke: } 5.5 \text { blocks per minute ( } 18 \% \\
\text { percent change) [48]; Spastic Hemiplegia: } 4 \text { blocks } \\
\text { per minute for } 2 \text { week training [50]. }\end{array}$ \\
\hline $\begin{array}{l}\text { Motor } \\
\text { Assessment } \\
\text { Scale (MAS) }\end{array}$ & $\begin{array}{l}\text { Test-Retest Evidence for } \\
\text { reliability of scores: Acute and } \\
\text { chronic stroke: } r=0.87 \text { to } 1.00 \\
\text { [55] }\end{array}$ & $\begin{array}{l}\text { Criterion-related evidence for validity of } \\
\text { scores: Acute stroke: Concurrent with } \\
\text { modified FMA (rho }=0.96 \text { ) [56], similar } \\
\text { MAS to FMA items rho }=0.65 \text { to } 0.93 \text { except }\end{array}$ & $\begin{array}{l}\text { Stroke: ES: } 0.36 \text { to } 0.5 \text { [59], Subjects with no change } \\
\text { on the measure } 44.4 \text { to } 63.9 \% \text { [59], MAS arm items - } \\
80 \% \text { of subjects rated at the extremes of the scales } \\
\text { [59]. Significant differences in mean scores } 71 \text { days }\end{array}$ \\
\hline $\begin{array}{l}\text { Carr et al., } \\
1985 \text { [55] }\end{array}$ & $\begin{array}{l}\text { Inter-rater evidence for } \\
\text { reliability of scores: Acute } \\
\text { stroke } 87 \% \text { agreement [55] }\end{array}$ & $\begin{array}{l}\text { FMA balance items }(\text { rho }=0.12)[56] . \\
\text { Construct-related evidence for validity of } \\
\text { scores: Acute stroke: Convergent: MAS and } \\
\text { functional balance test, sitting arm raise }(r= \\
0.33) \text { and sitting forward reach }(r=0.54)[57] \text {, } \\
\text { Mobility Scale for Acute Stroke mobility } \\
\text { items convergent with MAS } r=0.89 \text { [58]. }\end{array}$ & $\begin{array}{l}\text { after rehabilitation for stroke [60]. Floor/Ceiling } \\
\text { effects: Stroke: Floor effects on admission for upper } \\
\text { arm function }(18 \%) \text {, hand movements }(41 \%) \text { and } \\
\text { advanced hand activities }(47.5 \%) \text {. Floor effects on } \\
\text { discharge for hand movements }(27.9 \%) \text { and } \\
\text { advanced hand activities }(36.1 \%) \text {. } \\
\text { Stroke: Ceiling effects on admission for upper arm } \\
\text { function }(19.7 \%) \text {, hand movements }(18.0 \%) \text {. Ceiling } \\
\text { effects on discharge for upper arm function (41\%), } \\
\text { hand movements }(36.1 \%) \text {, advanced hand activities } \\
(16.4 \%)[59] \text {. }\end{array}$ \\
\hline
\end{tabular}


Table 2.4. Table of Evidence: Performance-based Measures Psychometric Properties (Continued)

\begin{tabular}{|c|c|c|c|}
\hline $\begin{array}{l}\text { Measure } \\
\quad \text { Author(s), year }\end{array}$ & Evidence for reliability of scores & Evidence for validity of scores & Evidence for responsiveness \\
\hline $\begin{array}{l}\text { Nine Hole Peg } \\
\text { Test (NHPT) } \\
\\
\text { Heller et al., } \\
1987 \text { [61] }\end{array}$ & 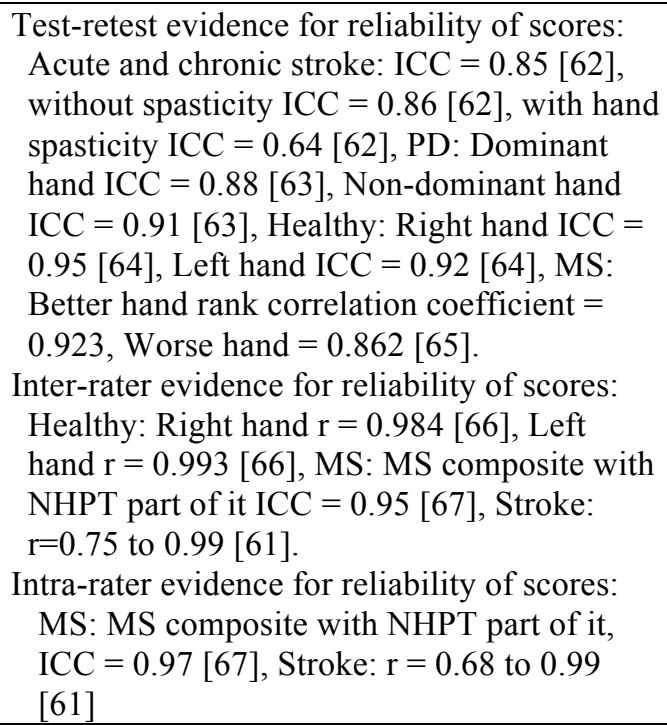 & $\begin{array}{l}\text { Criterion-related evidence for validity of scores: } \\
\text { Concurrent evidence for validity of scores: Stroke: } \\
\text { Poor concurrent with Frenchay Arm test }-27 \% \\
\text { cases improperly grouped [68]. Stroke: Concurrent } \\
\text { with SIS-HD pre-treatment (rho }=0.58 \text { ), post- } \\
\text { treatment (rho }=-0.66 \text { ) [69], BBT and ARAT pre- } \\
\text { treatment (rho }=-0.55 \text { to }-0.80 \text { ) and post-treatment } \\
\text { (rho }=-0.57 \text { to }-0.71 \text { ) [68]. FMA and MAL pre- } \\
\text { treatment (rho }=-0.16 \text { to }-0.27 \text { ), post-treatment (rho } \\
=-0.18 \text { to }-0.33 \text { ) [68]. Healthy: Concurrent with } \\
\text { Purdue Pegboard Test (rho }=-0.74 \text { to }-0.75 \text { ) [64]. } \\
\text { Predictive evidence for validity of scores: Poor at } 1 \\
\text { month [68]. } \\
\text { Construct-related evidence for validity of scores: } \\
\text { Stroke: Convergent with Motricity Index r }=0.82 \text {. }\end{array}$ & $\begin{array}{l}\text { Stroke: ES at } 1 \text { and } 3 \text { months post } \\
\text { stroke }=0.52,1 \text { and } 6 \text { months } \\
\text { post stroke }=0.66 \text { [70], SRM = } \\
0.64 \text { to } 0.79 \text { [69] MDC: Stroke }= \\
32.8 \text { seconds (Percent change }= \\
54 \% \text { ) [62], PD: MDC Dominant } \\
\text { hand = 2.6 seconds, Non- } \\
\text { dominant hand = } 1.3 \text { seconds } \\
\text { [63]. } \\
\text { Floor effect: Stroke: } 100 \text { second } \\
\text { cut-off score }-20 \% \text { with } \\
\text { minimum score at discharge } \\
\text { [71], 50 second cut-off score - } \\
\text { poor floor effects [68] }\end{array}$ \\
\hline $\begin{array}{l}\text { Sollerman Hand } \\
\text { Function Test } \\
\text { (SHFT) } \\
\text { Sollerman et al., } \\
1995 \text { [72] }\end{array}$ & $\begin{array}{l}\text { Test-Retest Evidence for reliability of scores: } \\
\text { Chronic Stroke ICC }=0.96-0.98[73] \\
\text { Chronic Burns: Inter-rater evidence for } \\
\text { reliability of scores ICC }=0.98, \text { Intra-rater } \\
\text { evidence for reliability of scores ICC }=0.98 \\
\text { [74]. Chronic Stroke: Inter-rater ICC }=0.96 \\
\text { - } 0.98, \text { Intra-rater evidence for reliability of } \\
\text { scores ICC }=0.96-0.99[73]\end{array}$ & $\begin{array}{l}\text { Criterion-related evidence for validity of scores: } \\
\text { Tetraplegia: Concurrent with subjective hand } \\
\text { function on visual analog scale }(\mathrm{r}=0.68) \text {, Disability } \\
\text { Rating Scale for insurance purposes in Sweden for } \\
\text { hand dysfunction }(\mathrm{r}=0.88)[72] \text {. Gout: Concurrent } \\
\text { with hand tophus joint }(\mathrm{r}=0.59)[75] \\
\text { Construct-related evidence for validity of scores: } \\
\text { Tetraplegia: Convergent with Motor Capacity Scale } \\
(\mathrm{r}=0.959) \text {, Gout: Convergent with Grip strength ( } \mathrm{r} \\
=0.731)[75] \text {, DASH }(\mathrm{r}=0.887)[75] \text {. }\end{array}$ & $\begin{array}{l}\text { MDC: Chronic Burns }=6.7-6.9 \\
{[74]} \\
\text { Chronic Burns: SEM }=2.6[74]\end{array}$ \\
\hline
\end{tabular}


Table 2.4. Table of Evidence: Performance-based Measures Psychometric Properties (Continued)

\begin{tabular}{|c|c|c|c|}
\hline $\begin{array}{l}\text { Measure } \\
\text { Author(s), year }\end{array}$ & $\begin{array}{l}\text { Evidence for reliability of } \\
\text { scores }\end{array}$ & Evidence for validity of scores & Evidence for responsiveness \\
\hline $\begin{array}{l}\text { Test Evaluant la } \\
\text { Performance des } \\
\text { Membres } \\
\text { supérieurs des } \\
\text { Personnes âgées } \\
\text { (TEMPA) } \\
\quad \text { Desrosiers et } \\
\text { al, } 1995[76]\end{array}$ & $\begin{array}{l}\text { Test-Retest Evidence for } \\
\text { reliability of scores: Older } \\
\text { adults: ICC } 0.70 \text { to } 1.0[76] \\
\text { Inter-rater evidence for } \\
\text { reliability of scores: Older } \\
\text { adults: Non-simultaneous } \\
\text { inter-raters ICC }=0.68 \text { to } 1.0 \text {, } \\
\text { simultaneous inter-raters }= \\
0.96 \text { to } 1.0 \text {. }\end{array}$ & $\begin{array}{l}\text { Criterion-related evidence for validity of } \\
\text { scores: MS: Concurrent with JHFT } \mathrm{r}=0.56 \text { to } \\
0.87 \text { [77], NHPT } \mathrm{r}=0.79 \text { to } 0.9, \mathrm{FIM} \mathrm{r}=0.44 \\
\text { and ADL self-questionnaire } \mathrm{r}=0.61[77] . \\
\text { Construct-related evidence for validity of } \\
\text { scores: Convergent with functional } \\
\text { independence in basic personal care (rho }= \\
0.74 \text { ) }\end{array}$ & Not reported \\
\hline $\begin{array}{l}\text { Arm Motor } \\
\text { Ability Test } \\
\text { (AMAT) } \\
\text { Kopp et al., } \\
1997[78]\end{array}$ & $\begin{array}{l}\text { Test-retest evidence for } \\
\text { reliability of scores: Stroke: } \\
\text { ICC } 0.93-0.99 \text { [78] } \\
\text { Inter-rater evidence for } \\
\text { reliability of scores: Stroke: } \\
\text { ICC }=0.95-0.99[78]\end{array}$ & $\begin{array}{l}\text { Criterion-related evidence for validity of } \\
\text { scores: Stroke: Concurrent with FMA }=0.92- \\
0.94 \text { [79] } \\
\text { Construct-related evidence for validity of } \\
\text { scores: Stroke: Convergent with MI-UE r }= \\
0.45-0.61 \text { [78] }\end{array}$ & $\begin{array}{l}\text { Stroke: } 1 \text { versus } 2 \text {-week change detected in subacute } \\
\text { stroke for mild to moderate movement deficits [78]. }\end{array}$ \\
\hline $\begin{array}{l}\text { Stroke } \\
\text { Rehabilitation } \\
\text { Assessment of } \\
\text { Movement Scale } \\
\text { (STREAM) } \\
\quad \text { Daley et al., } \\
\text { 1999 [80] }\end{array}$ & $\begin{array}{l}\text { Test-Retest Evidence for } \\
\text { reliability of scores: Chronic } \\
\text { Stroke: Mobility subscale ICC } \\
=0.96 \text { (0.93 to } 0.98) \text { [81] } \\
\text { Chronic Stroke: Inter-rater } \\
\text { evidence for reliability of } \\
\text { scores UE }=0.994, \text { Total }= \\
\text { 0.995. Intra-rater UE = } \\
\text { 0.963, Total }=0.999[80] . \\
\text { Chronic Stroke: Cronbach's } \\
\text { alpha overall score }=0.984, \\
\text { limb subscales }=0.979[80]\end{array}$ & $\begin{array}{l}\text { Criterion-related evidence for validity of } \\
\text { scores: Acute stroke: Concurrent with } \\
\text { STREAM - UE with Box and Block affected } \\
\text { UE initial }=0.78 \text { and } 3 \text { months }=0.76 \text {. } \\
\text { Unaffected UE initial }=0.31 \text { and } 3 \text { months }= \\
0.31 \text { [82]. } \\
\text { Construct-related evidence for validity of } \\
\text { scores: Acute stroke: Convergent with Barthel } \\
\text { Index } 14 \text { days }=0.80 \text { at } 14 \text { days and } 180 \text { days }= \\
0.76 . \text { Predictive with Barthel Index } 0.54 \text { at } 14 \\
\text { days and } 0.81 \text { at } 90 \text { days. } \\
\text { Face validity evidence: } 20 \text { physical therapists } \\
\text { reviewed and refined the measure [80] }\end{array}$ & 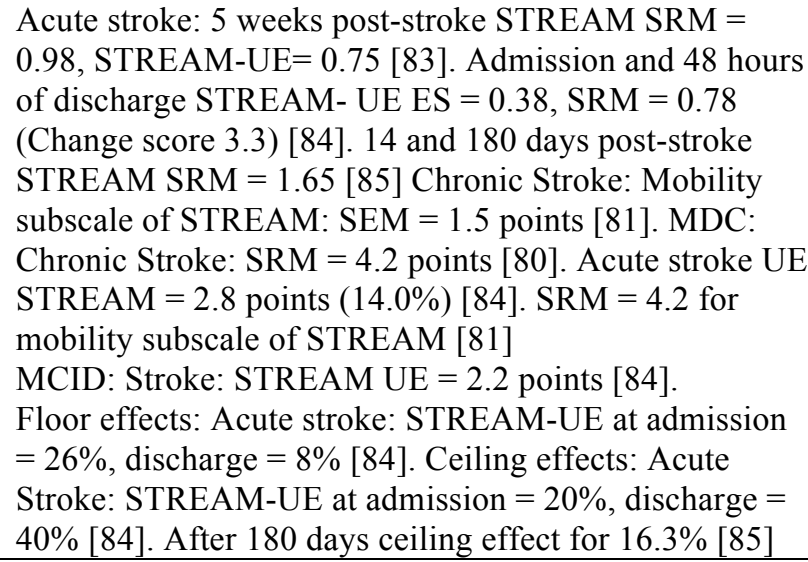 \\
\hline
\end{tabular}


Table 2.4. Table of Evidence: Performance-based Measures Psychometric Properties (Continued)

\begin{tabular}{|c|c|c|c|}
\hline $\begin{array}{l}\text { Measure } \\
\text { Author(s), } \\
\text { year }\end{array}$ & $\begin{array}{l}\text { Evidence for reliability of } \\
\text { scores }\end{array}$ & Evidence for validity of scores & Evidence for responsiveness \\
\hline $\begin{array}{l}\text { Wolf Motor } \\
\text { Function } \\
\text { Test } \\
\text { (WMFT) } \\
\quad \text { Wolf et } \\
\text { al., 2001 } \\
\text { [86] }\end{array}$ & $\begin{array}{l}\text { Test-Retest evidence for } \\
\text { reliability of scores: Chronic } \\
\text { TBI: ICC } 0.97(0.89 \text { to } \\
0.97) \text { [87], Chronic stroke r } \\
=0.95, \text { ICC }=0.97 \text { [87]. } \\
\text { Inter-rater evidence for } \\
\text { reliability of scores: Chronic } \\
\text { stroke: ICC } 0.93 \text { to } 0.99 \\
\text { [88] }\end{array}$ & $\begin{array}{l}\text { Criterion-related evidence for validity of scores: } \\
\text { Stroke: Concurrent with FMA-UE r }=-0.57 \text { to - } \\
0.88 \text { [86] } \\
\text { Construct-related evidence for validity of scores: } \\
\text { Stroke: Known group evidence for validity of } \\
\text { scores of scores: WMFT score for individuals } \\
\text { without impairment were significantly higher } \\
\text { than clients with stroke for both dominant and } \\
\text { non-dominant hand [86] }\end{array}$ & $\begin{array}{l}\text { SRM: Acute stroke: Time }=0.38 \text {, functional ability } \\
\text { scale (FAS) }=1.30 \text { [89] SEM: } 0.2 \text { seconds [90], } \\
\text { Evidence for MDC: Chronic stroke: Time: } 0.7 \\
\text { seconds, FAS: } 0.1 \text { points [90]. MCID: Acute } \\
\text { stroke: FAS dominant side affected }=1.0(17 \% \\
\text { change), time dominant side affected }=-19.0 \\
\text { seconds (16\% change) [91] } \\
\text { Evidence for Ceiling effects: Chronic stroke: } 5 \text { to } \\
17 \% \text { of patients [92] }\end{array}$ \\
\hline $\begin{array}{l}\text { Chedokee } \\
\text { Arm and } \\
\text { Hand } \\
\text { Inventory } \\
\text { (CAHAI) }\end{array}$ & $\begin{array}{l}\text { Test-retest evidence for } \\
\text { reliability of scores: UE } \\
\text { paralysis: ICC }=0.96[94] \\
\text { Inter-rater evidence for } \\
\text { reliability of scores: UE } \\
\text { paralysis: ICC }=0.98[94] \\
\text { Cronbach's alpha }=0.98 \\
\text { [93] }\end{array}$ & $\begin{array}{l}\text { Criterion-related evidence for validity of scores: } \\
\text { Concurrent evidence with ARAT, CMSA, ROC } \\
\text { curves CAHAI - 7=0.95, CMSA }=0.76, \text { ARAT } \\
=0.88 \text { [95] } \\
\text { Construct-related evidence for validity of scores: } \\
\text { Known groups evidence: ROC curves for CAHAI } \\
-13=0.86, \text { CAHAI }-9=0.82 \text { greater than } \\
\text { ARAT }=0.72[95] . \text { Content/Face validity } \\
\text { evidence: Reported for stroke } 94]\end{array}$ & $\begin{array}{l}\text { Stroke: ROC curves for CAHAI }-13=0.86, \\
\text { CAHAI }-9=0.82 \text { were greater than ARAT }= \\
0.72 \text {, demonstrating sensitivity of CAHAI to } \\
\text { change }[94] . \text { UE paralysis MDC }(90)=6.3 \text { points } \\
{[94]}\end{array}$ \\
\hline
\end{tabular}

Note: ADL: Activities of Daily Living; CMSA: Chedokee McMaster Stroke Assessment; CTS: Carpal Tunnel Syndrome; ES: Effect Size; FIM: Functional Independence Measure; ICC: Intraclass Correlation Coefficient; MCID: Minimal Clinically Important Difference; MDC: Minimal Detectable Change; MHQ: Michigan Hand Questionnnaire; OA: Osteoarthritis; RA: Rheumatoid Arthritis; ROC: Receiver Operating Curve; SCI: Spinal Cord Injury; SEM: Standard Error of Measurement; SRM: Standard Response Mean; TBI: Traumatic Brain Injury; UE: Upper Extremity; [1] Jebsen et al., 1969 ; [2] Beebe \& Lang, 2009 ; [3] Ferreiro et al., 2010 ; [4] Hackel, Wolfe, Bang, \& Canfield, 1992 ; [5] Sears \& Chung, 2010 ; [6] Lincoln \& Leadbitter, 1979 ; [7] Kurtaiş et al., 2009 ; [8] Endres, Nyary, Banhidi, \& Deak, 1990 ; [9] Collin \& Wade, 1990 ; [10] Soyuer \& Soyuer, 2005 ; [11] Sackley \& Lincoln, 1990 ;[12] Collen, Wade, \& Bradshaw, 1990 ; [13] Williams, Robertson, Greenwood, Goldie, \& Morris, 2006 ; [14] Sunderland et al., 1989; [15] Collin \& Wade, 1990 ;[16] Bohannon, 1999 ;[17] Vos-Vromans, de Bie, Erdmann, \& van Meeteren, 2005; [18] Lyle, 1981 ; [19] Platz et al., 2005; [20] Nijland et al., 2010; [21] van der Lee, Beckerman, Lankhorst, \& Bouter, $2001 ;$ [22] Platz et al., $2005 ;[23]$ Yozbatiran et al., 2008; [24] Lin, Wu, Liu, Chen, \& Hsu, 2009;[25] Beebe \& Lang, 2009 ; [26] Lang, Wagner, Dromerick, \& Edwards, 2006 ; [27] Duncan et al., 1983 ; [28] Platz et al., 2005 ; [29] Lin, Hsueh, Sheu, \& Hsieh, 2004;[30] Wood-Dauphinee, Williams, \& Shapiro, 1990 ;[31] Malouin et al., 1994 ; [32] Duncan, Goldstein, Matchar, Divine, \& Feussner, 1992 ; [33] Mao, Hsueh, Tang, Sheu, \& Hsieh, 2002 ; [34] Shelton, Volpe, \& Reding, $2001 ;$;35] Bernspång, Asplund, Eriksson, \& Fugl-Meyer, 1987 ;[36] Dettmann, Linder, \& Sepic, 1987 ; [37] Hsieh et al., 2009; [38] Woodbury et al., 2008; [39] Crow \& Harmeling-van der Wel, 2008 ; [40] Gladstone et al., 2002 ; [41] van der Lee et al., 2001 ;[42] Hsueh et al., 2008 ;[43] Wagner, Rhodes, \& Patten, 2008; [44] Shelton, Volpe, \& Reding, 2001 ; [45] Wilson et al., 1984 ;[46] Fong et al., 2004 ; [47] Mathiowetz et al., 1985 ;[48] Chen, Chen, Hsueh, Huang, \& Hsieh, 2009 ; [49] Desrosiers, Bravo, Hébert, \& Dutil, 1995 ; [50] Siebers, Oberg, \& Skargren, 2010 ; [51] Canny, Thompson, \& Wheeler, 2009 ; [52] Platz et al., 2005 ; [53] Lin, Chuang, Wu, Hsieh, \& Chang, 2010 ; [54] Higgins et al., 2005; [55] Carr et al., 1985;[56] Malouin et al., 1994 ; [57] Tyson \& DeSouza, $2004 ;[58]$ Simondson, Goldie, \& Greenwood, 2003; [59] English et al., 2006; [60] Dean \& Mackey, 1992; [61] Heller et al., 1987; [62] Chen et al., 2009; [63] Earhart et al., 2011 ; [64] Wang et al., 2011 ; [65] Erasmus et al., 2001 ; [66] Oxford Grice et al., 2003 ; [67] Cohen et al., 2000 ; [68] Sunderland et al., 1989; [69] Lin, Chuang, et al., 2010;[70] Beebe \&

Lang, 2009 ; [71] Jacob-Lloyd \& Dunn, 2005 ; [72] Sollerman \& Ejeskär, 1995 ; [73] Brogårdh \& Sjölund, 2006 ;[74] Weng et al., 2010; [75] Dalbeth et al., 2007 ; [76] Desrosiers, Hébert, et al., 1995; [77] Feys et al., 2002 ; [78] Kopp et al., 1997a ; [79] Chae et al., 2003 ; [80] Daley et al., 1999 ; [81] Chen et al., 2007 ; [82] Ahmed et al., 2003 ; [83] Higgins et al., 2005; [84] Hsueh et al., 2008 ; [85] Hsueh, Wang, Sheu, \& Hsieh, 2003 ; [86] Wolf et al., 2001 ; [87] Shaw et al., 2005 ; [88] Morris et al., 2001 ; [89] Hsieh et al., 2009;[90] Fritz, Blanton, Uswatte, Taub, \& Wolf, 2009; [91] Lang et al., 2008 ; [92] Nijland et al., 2010 ; [93] Barreca et al., 2004 ; [94] Barreca et al., 2005 ; [95] Barreca et al., 2006 
Table 2.5. New Measures of Hand and Arm Function Identified in the Review

\begin{tabular}{lll}
\hline Measure & $\begin{array}{l}\text { International Classification of } \\
\text { Functioning, Disability and } \\
\text { Health category }\end{array}$ & Laterality \\
\hline Self-report & $\begin{array}{l}\text { Activity and Participation } \\
\text { Activity }\end{array}$ & $\begin{array}{l}\text { Unimanual } \\
\text { Unimanual }\end{array}$ \\
$\begin{array}{l}\text { Hand Function Survey } \\
\text { Reaching self efficacy (RSE), camera recording } \\
\text { during testing }\end{array}$ & $\begin{array}{l}\text { Body structure and function } \\
\text { Performance }\end{array}$ & Bimanual \\
\hline 16-Hole Peg Test & Activity & Unimanual \\
Extended Drawing Test (computerized) & Activity & Unimanual \\
Manual Function test & Activity & Unimanual \\
Upper Body Dressing Scale for buttoned shirt & Activity & Bimanual \\
\hline
\end{tabular}




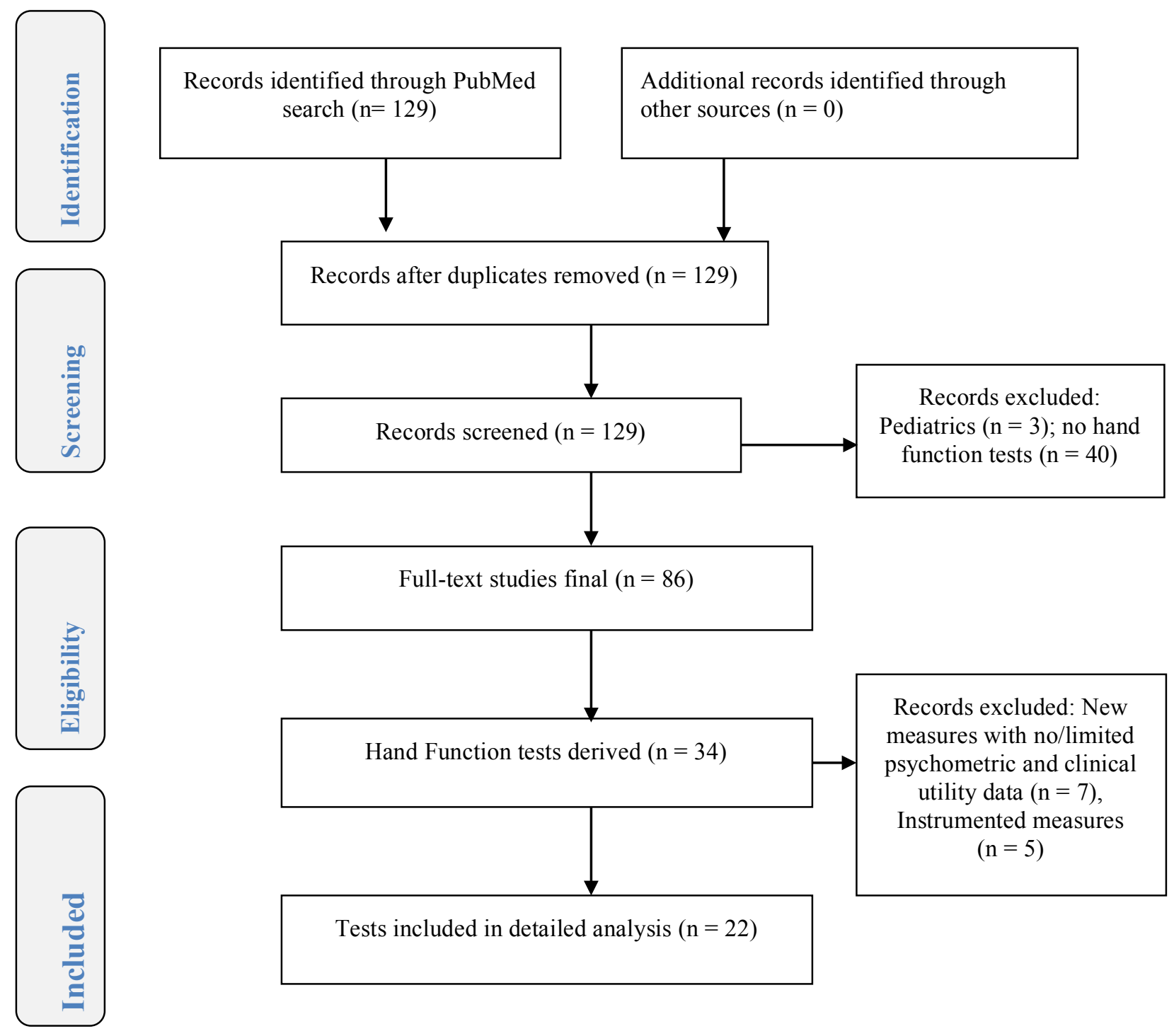

Figure 2.1. PRISMA Flow Diagram 


\title{
Chapter 3: Construct Definition and Expert Panel Study
}

\author{
Abstract \\ Background and Purpose: A new measure - Hand and Arm Function Measure (HAFM) \\ - was developed for assessing upper extremity function in daily activities in people with \\ neurological conditions. This clinical measure has a combination of self-reported and \\ performance-based items. The conceptual rehabilitation framework of International \\ Classification of Functioning, Disability and Health (ICF) and the measurement framework of \\ evidence-centered design (ECD) shaped the development of this measure. An expert panel study \\ was undertaken to shed light on the definition of the construct, item development, and item \\ writing.
}

Method: The construct of 'hand and arm function in daily activities' is defined for the purpose of this measure using a construct map and the relationships to other variables are described using the nomological network. Experts were involved in two stages: Construct and item bank development and item writing and modification to establish face and content-related evidence for validity of scores. The four experts included people in clinical practice of physical and occupational therapy in neurological conditions, teaching, research, and measurement. The construct and item bank development stage involved a qualitative descriptive study with the focus group of experts. The item writing and modification was conducted after patient/client focus groups and was in the form of an open-ended questionnaire. The identified items were examined, suggestions for item addition or deletion were made by the experts, and their feedback was incorporated.

Results and conclusion: The construct of 'hand and arm function in daily activities' was defined, item bank development started and item modifications were considered. The construct 
map, nomological net and expert panel study informed the development of the first iteration of the self-reported and performance-based sections of the Hand and Arm Function Measure. 


\section{Construct Definition}

Introduction. Hand and arm function has varied perspectives for developing an operational definition. The perspectives differ mainly in the extent of proximal function involved in the conceptualization. The definition could include reflexes as defined in Fugl-Meyer Assessment Scale - Upper Extremity Section (FMA-UE) (Duncan et al., 1983). It could include more proximal body functions like scapular function in Stroke Rehabilitation Assessment of Movement - Upper Extremity Section (STREAM-UE) (Daley et al., 1999). It can take into account shoulder function as defined in the measure DASH (Hudak et al., 1996). It could take into account grip strength as defined in Jamar® dynamometer (Mathiowetz et al., 1985). It could take into account only wrist and hand function as defined in the measure Nine Hole Peg Test (NHPT) (Oxford Grice et al., 2003). There is no published operational definition of the construct for most of the hand and arm function measures in literature, except for Motor Activity Log (MAL) that claims to measure 'real world hand use' (Uswatte, et al., 2005).

Defining the construct operationally provides a framework for analysis of the concept to be measured (Ketterlin-Geller, Yovanoff, Jung, Liu, \& Geller, 2013). Some authors doubt the utility of creating a general definition of the construct (Nesselroade, Gerstorf, Hardy, \& Ram, 2007). The concern relates to the individual differences among people that cannot be measured with a generic construct. For example, low level of the trait of hand and arm function could mean poor ability to do tasks needing strength for one individual or high pain in the wrist for another individual. This concern cannot be alleviated easily, however defining all the possible combinations leading to a low level and high level of trait can clarify the understanding and use of the scores. Even though, hand and arm function appears to be a simple construct, it has many aspects that can be measured (e.g., grip strength, sensation, dexterity, etc.) making it a complex 
construct to operationally define. Thus, the construct definition needs to take into consideration the wide range of dimensions to be measured in developing an operational definition (Sadler, 2003).

Objective. The objective of this study was to define and describe the construct of 'hand and arm function in daily activities' using a construct map and the relationships to other variables using a nomological network.

Definition. Good or high trait of hand and arm function was defined as the ability of a person to participate in a wide range of daily activity tasks with the use of their hands and arms with optimal speed and quality, without experiencing interference by body structure and function problems when the environmental conditions remain constant. The guiding principles for development of the measure include: a) combination of self-reported and performance-based items, b) feasibility to administer in a clinical setting, c) evidence for reliability of scores for measuring high and low trait of hand function in neurological conditions, and d) sensitive to change.

This construct has two dimensions, gross and fine movements in daily activities based on current literature (Lehman et al., 2011). 'Gross movements in daily activities' dimension is defined as proximal arm movements such as transport, reaching, carrying, and pushing. Transport and reach is typically performed in combination with prehension. Pushing and carrying may not accompany prehension. 'Fine movements in daily activities' dimension was defined as prehensile movements like grip, and non-prehensile skilled movements like turning and pointing. Fine movements in daily activities for the purpose of this measure includes sensory function that is essential for hand function. The sensory task that is used in the performancebased measure involves fine movements of small objects in a sock without visual input and thus 
falls within the fine movements dimension. The hand functions were defined with Jones and Lederman's classification system (Jones \& Lederman, 2006) described previously in Chapter 2. Although, the hypothesized dimensions in the measure are gross and fine movements, it is possible that the dimensions of body structure and function, and activity and participation based on International Classification of Functioning, Disability and Health (ICF) (WHO, 2001) may emerge predominantly. ICF framework was the theoretical framework selected for development of this measure. The acknowledgement of this possibility is important during factor analysis studies in the later stages of development of the measure when the dimensionality will be explored statistically. As the measure is based on ICF and the rating of symptoms within the self-report section may not fit neatly into gross and fine movement categories, further investigation of the domains may be needed.

ICF framework. The World Health Organization's International Classification of Functioning, Disability and Health (ICF) (WHO, 2001) provides an common taxonomy of health-related domains. The ICF core set for hand conditions was used as a broad framework for the self-reported measure (Coenan, 2015). An European Delphi Study has taken this core set and identified the existing impairment and activity measures related to hand and arm function that will help assess the aspects in the core set (van de Ven-Stevens et al., 2016). This approach however sets up a clinician for using multiple measures to assess aspects of the hand and arm core set as opposed to the current measure that attempts to unify the measurement process to one single measure. The core set provides lists of categories that are relevant for hand function and disability to support the application of ICF in practice. It takes into account all the possible areas within body structure, body function, activity and participation that are influenced by hand function problems. Although, hand function is affected by environmental and personal contexts 
as outlined in the core set, the Hand and Arm Function Measure assumes these factors are constant when measuring body function, activity and participation. Table 3.1 provides the construct schema in detail with the representation of ICF categories, hand function domains and corresponding items in the Hand and Arm Function Measure.

Evidence Centered Design (ECD) Framework. The ECD framework is a measurement framework that enables systematic study of the development of the new measure (Mislevy et al., 2003). The categories in ECD framework are discussed previously in Chapter 1. Note that these categories can overlap and multiple iterations of the processes can exist. The layers of ECD and their application for the Hand and Arm Function Measure are summarized in Table 3.2 and subsequently described.

Domain analysis. The content, concepts and representations of hand and arm function are studied in domain analysis and have undergone revisions over the course of this study. For example, it was expected for the name of the measure to reflect the purpose. The original name for the Hand and Arm Function Measure was 'fine motor function measure.' As the work to accumulate evidence for the background related to the various components of hand function developed, the domain of fine motor no longer adequately reflected the purpose. Fine motor control (e.g., dexterity related movements such as picking up coins) was also analyzed theoretically to have minimal value in the absence of gross motor (e.g., arm, forearm and hand combined movements such as reaching for a glass on a shelf) information to have meaning for the interpretation of scores. Moreover, the term 'motor' underrepresented the varied domains underlying movements such as sensation, control, and coordination. The revised domain thus emerged to be one of 'real world hand use' and since it is not feasible to 'observe' real world performance, this was revised to 'hand use.' The need to change this further to reflect 'arm and 
hand use' was considered to accurately reflect what aspects of hand function the measure supported. Expert and participant (individuals with neurological conditions) input further revised this to fit the ICF model and the name of the construct was changed to its current form of "hand and arm function in daily activities.' The evidence for reliability of scores for quantifying hand use was a concern expressed by expert participants. The ambiguity of the term 'use' with the implication of 'use for what purpose' was expressed by participants with neurological conditions. Moreover, the term 'upper extremity' although better fits the name of the measure for the therapists, it is not the terminology that the patients/clients can identify with. Thus, this patient/client centered measure used easily understood terms of 'hand and arm' to covey the gross and fine movement aspects to the patients/clients.

A nomological network or net is a schematic description of the factors that affect the construct 'hand and arm function in daily activities' that explains the relationships of the aspects within and outside of the construct. It has been used in measurement for many decades (Cronbach \& Meehl, 1955). The nomological network for HAFM is shown in Figure 3.1 and is organized with the ICF framework as the overarching framework with the domains under each category shown with their relationship with hand and arm function. The solid lined text boxes in Figure 3.1, indicate the continuum from the health conditions that this construct addresses that affect the person's body function resulting in hand and arm function problems that manifest in the activity and participation domains tested in this measure (Lemmens et al., 2012). The dashed lined boxes indicate the other body structure and function, environment, and personal factors that can affect the construct. Other health conditions affecting the construct and changes in the demands of the task are other considerations not shown in the figure that can affect the factors in 
this nomological network. Although evidence such as that provided by Lemmens et al., (2012) to support each relationship in this network is desirable, deeper research is lacking in this field.

The construct map is shown in Figure 3.2. It explains the organization of the construct to reflect high to low function and how that will correspond to the change in scores and level of difficulty on the measure. High scores on the measure indicate better function or high level of the trait of hand and arm function. Low scores on the measure indicate poor hand and arm function.

Domain modeling. The Toulmin diagrams for assessment arguments (Mislevy et al., 2003) and 'design patterns' (Mislevy, 2011) are the ways in which domain modeling was represented. Table 3.2 gives the design pattern attributes with the corresponding terminology in Messick's Assesment Argument and Toulmin Assessment Argument with the application to the HAFM. The Toulmin Assessment Arguments can be demonstrated with a diagram for Hand and Arm Function Measure as shown in Figure 3.3. The warrant is the evidence for the claim made by the measure. Identifying these warrants and claims helps clarify the intentions of the HAFM. The construct definition has undergone changes over the course of this study. These changes are summarized in Table 3.3. The changes not only impact the design of the assessment but they also impact the studies designed for testing evidence for validity of scores of the measure. The major changes have been with the change in focus from purely fine motor to fine and gross movements. The target population was also clearly defined and its effect on the design of the evidence for validity of scores was included to reduce construct under-representation (e.g., by including wider population) and construct irrelevant variance (e.g., by measuring aspects not related to the construct). 
Conceptual Assessment Framework. This process organizes the purpose, practical or financial limits in order to create the framework of the test. This framework is organized into three perspectives (Messick, 1994), student (patient/client), task and evidence.

a) Student (patient/client) model. In the student model, the claims of patient/client's knowledge and skills that can be made by the interpretation of test scores are addressed. For the Hand and Arm Function Measure, we have evidence from prior literature that disorders can cause impairments in the sub-domains of strength, sensation, mobility, stability, pain and coordination which can cause subsequent difficulty in function such as in reach, grasp, release, in-hand manipulation, unilateral and bilateral control (Radomski \& Trombly, 2007). These patient/client-related variables can vary and contribute to the information we can get about the patient/client from the scores.

b) Task model. The tasks that should elicit the behaviors are addressed in this model. The environment that influences the participant is studied as it affects the performance on the measure. The evidence behind using object manipulation tasks for the Hand and Arm Function Measure falls into this category (Hsieh et al., 2009; Jebsen, Taylor, Trieschmann, Trotter, \& Howard, 1969). The concept of introducing gradation of tasks in the Hand and Arm Function Measure based on the function and control sub-domains is an element of the task model. A change in domain modeling to include reach, grasp, release, in-hand manipulation, unilateral and bilateral control also impacted the task model which now has to consider a more broader collection of items to address these sub-domains.

c) Evidence model. An evidence model connects the student (patient/client) and task models. The behaviors that should reveal the construct are translated into scores. This has two components, evaluation and measurement. The evaluation component addresses the scoring 
rules, for example, manual versus computer based. Also, decisions such as scoring rubrics are made within the evaluation component. For the Hand and Arm Function Measure, the observed tasks have a scoring form that relates to completion of task levels and compensations used during task, this rubric can help generate scores. For the self-report items, the current version has a paper and pencil single version format with response choices related to interference of hand and arm function issues or symptoms with daily tasks. The measurement component includes decisions about using statistical models such as classical test theory models or the more recent item response theory (IRT) models. The selection of classical test theory model for Hand and Arm Function Measure was made considering the moderate sample size estimates for the neurological population for the pilot study. The scores in the two sections of the hand and arm function measures would be combined. Cluster analysis would be desired to identify normal, mild, moderate and severe impairment categories. According to the Code of Fair Testing Practices in Education (Joint Committee on Testing Practices, 2004) informing test takers about results is considered a standard in education. However, clinics are high stakes testing situations where client's adverse responses or indifference to information about the results can be difficult to handle. Many clinicians choose not to share the results with clients unless specifically requested by clients. This practice needs to change in order to be more transparent with our clients and share the results in a meaningful way. Thus, this new measure incorporates this belief and ways to inform the clients about the results.

Assessment implementation. This involves the actual constructing of the test and its operational elements. For the Hand and Arm Function Measure, the self-report items were put together in a format with ease of reading, reading level and clarity of instructions under consideration. The writing of items is another process that sought expert input. The observation 
task rules were generated however the test materials are still under development in this phase. Moreover, as domain analysis and modeling become more formed and clear, the actual test construction undergoes changes at every stage. For example, the response choices for the selfreport items in the prior version were 'none of the time,' 'some of the time,' 'most of the time,' and 'all the time.' However, after putting the items together in the form of a questionnaire, it was noted that some people never engaged in those tasks that were asked in the questionnaire. The responses were then revised to be able to mark 'N/A' to capture this information that could have potentially introduced irrelevant information in the test. Also, the response choices were changed to 'not at all,' 'a little,' and 'quite a lot.' The total scores were reflected to accommodate N/A and missed responses. To develop a good quality measure, there is a need to be constantly in the process of reducing construct-irrelevant information and under-representation that refines the construct definition and reiterates the process multiple times.

Assessment delivery. This is the process where the patient/client engages in the test. The four-process delivery architecture, discussed earlier in this paper, guides this process. Testing the Hand and Arm Function Measure with patients/clients would include the process of activity selection, presentation, collecting data, and accumulating evidence. The purpose of the test is always revisited in the process of making these decisions. The test results can be claimed to be useful within the studied diagnostic groups alone. Functional groups of mild, moderate and severe impairments based on scores may emerge that may be useful to distinguish one condition from another based on the spread of scores. If any claims will be made based on this information, the construct definition would then need to be reworked to address those uses. 


\section{Expert Panel Study}

Introduction. In order to develop a new measure of hand and arm function in neurological conditions, the evidence-centered design framework was adopted (Mislevy et al., 2003). According to the framework, the development of an item-bank is an important first stage along with the previously discussed construct definition. Experts in the field are the stakeholders who will use the measure and based on their experience have opinion about the development and use of a hand and arm function measure. Involving experts early on provides analysis of the construct, its operational definition and the development of an item bank.

Purpose. A qualitative descriptive study was undertaken to gain expert input on the definition of the construct of hand and arm function in daily activities, item development, and item writing. This was part of the first phase of development of the measure and consisted of four stages (refer to Figure 3.4 for schematic representation of the stages). Experts were involved in two stages: Stage I: Construct and item bank development and Stage III: item writing and modification to establish face and content-related evidence for validity of scores. Stage II and IV involved patient/client participants in focus groups and cognitive interviews respectively, and will be discussed in Chapter 4.

Method. A qualitative descriptive study research methodology using face-to-face semistructured format in a focus group was used to obtain in-depth data from experts in the field of occupational therapy, physical therapy and measurement. The pragmatic interview methodology was selected over surveys in order to help the participants understand the construct to be studied; to bring out hidden beliefs about assessment of arm and hand function, identify professional descriptors currently used in therapy and measurement; and to gather ideas about potential items through group validation. Human subjects approval was obtained from University of Washington 
Human Subject Division. A semi-structured interview guide (see Table 3.4) was developed based on literature regarding hand and arm function, the need to define the construct, and for item development. After informed consent, the focus group discussion was conducted at the University of Washington with four expert participants and two researchers $\left(\mathrm{NG}^{1}\right.$ and $\left.\mathrm{DK}^{2}\right)$. The interviews were audio-recorded with the informed consent of the participants and transcribed verbatim. A detailed account of the interview was maintained by the researcher.

Analysis. Data immersion, coding, category creation and thematic analysis were used to find patterns of meaning across data (Thomas, 2006; Schmidt, 2004). The researchers used an inductive approach to derive themes by interpreting the raw data. Coding categories and subcategories were allocated by two researchers independently. The researcher's comments were coded separate from the expert comments to prevent bias during coding. The researcher's notes after the session were not coded. The group discussion and item modification feedback was pooled for analysis. The data management software Dedoose version 6.2 was used for managing transcripts, codes and themes.

Results. The study recruited experts from the field of occupational therapy, physical therapy and measurement. To ensure that views from teaching and research in therapy were also represented, the participants were purposefully sampled. The sample size was limited by budget and participants were invited via a written and verbal invitation. Four expert participants were recruited with their demographic information provided in Table 3.5. There were two occupational therapists (OT), one physical therapist (PT) and one measurement expert. Of the two OTs, one had mainly clinical experience with adult neurological population, the other OT had a combination of teaching and clinical experience with children. The PT had clinical,

${ }^{1}$ NG: Namrata Grampurohit

${ }^{2}$ DK: Deborah Kartin 
research and teaching background with Parkinson disease population as the main area of interest with an added focus on fine motor function research. All three therapists have had experience with assessment and treatment of people with stroke. The measurement specialist had scale development, teaching and research background with research experience in rehabilitation. All expert participants approached agreed to participate in the study. All expert participants expressed interest in the measurement of arm and hand function and actively participated in the focus group. The audio-recordings were transcribed verbatim. There was overall good agreement $(89.35 \%)$ and the items of disagreement $(10.65 \%)$ were resolved by discussion to reduce researcher bias during thematic development phase. The disagreement was calculated by identifying excerpts marked and discussed by the researchers. Both researchers agreed on the final coding system and accepted it as being representative of the data. Seven key themes emerged and will be discussed in detail in the order in which they appear in the semi-structured interview guide.

Hand function versus hand use. The expert participants tried to differentiate between the two terms 'use' versus 'function.' They were able to identify hand function as related to 'properties' of the hand and use as specific skills that include function and bilateral use. However, they were not able to clearly point out a difference in their usage in the clinic. Moreover, they viewed both the terms under the activity and participation domain of the ICF (International Classification of Functioning, Disability and Health) with synonymous usage in clinic.

I pretty much use them synonymously, use and function. Just because I think I look at the framework that the ICF provides and I just put function or classify function in their activities domain. (E4) 
In view of the suggestion to ICF related terminology and unclear distinction between use and function, the name of the construct was changed from 'hand use' to 'hand function.'

Simulated versus real life tasks. Although the expert participants agreed that there is a difference between simulated versus real life tasks for assessment, the pros and cons of using both type of tasks were discussed. There was a desire to observe real life tasks because they are automatic and functional.

I think that it's always nice to see them do real life things because it's more automatic. So, that's kind of how I see that as more functional and the simulated is where they may not have ever done that before or it's not as automatic to them. (E1).

This theme validated the need of the self-report measure and it was evident that the focus would need to be real tasks that are common in daily activities. It was also discussed that compensation plays a role in real life tasks to get the tasks done efficiently and cannot be ignored or eliminated from the measure. However, experts in research emphasized the utility of simulated measures. I agree completely that doing the real thing is the real thing and cannot be substituted. But, I also think that sometimes a simulated test in a research environment does give you a lot of information that may be missed otherwise with a functional test. Like for example, if you were to look at force profiles with say an instrument that has force sensors on it and it simulates say jar opening. Especially, in the population that I am familiar with which is early Parkinson's disease. In their function, people may not show as many deficits especially if they are on medications. But with an instrumented device, you may see deficits in force profile that may not be evident on clinical examination. (E4)

Thus, the experts felt the need for sensitive measurement in the case of mild impairments, which validated the need for the performance section with simulated items that might be able to capture 
mild forms of impairment due to measurement of speed. However, since this measure is not designed for pure research but mainly for use in clinic, the instrumented measurement was not deemed necessary.

Compensations and adaptations. The experts agreed that people compensate in various ways such as stabilizing their postural muscles to bring about arm movements. Thus, the rating scale for the performance-based assessment should incorporate a category that includes compensations. Since adaptive devices and splints can be additionally applied to the upper extremity, the patient/client information section at the beginning of the self-report and performance-based measure would allow patients or therapist to enter this information. However, it was noted that some compensations can cause damage in the long run and it is important to identify them in assessment.

Or you feel that in the long term it may cause some other deficit like shoulder pain or something like that which you need to prevent at the right time. (E1)

The performance-based section of the new measure needs has to have provision for therapists to note such abnormal movements within the structure of the measure. Since these aberrations can be different for each individual and different for the various diagnoses, the rating scale cannot be specified to score lower for these movements.

There was a concern voiced about patient's perception of the performance-based assessment.

I think one of the items that missed in a lot of test is that we don't get an indication of how easy or difficult it is for the patient. (E4)

This concern indicated a need for patient-report in the performance-based assessment. It could not be incorporated for each item on the measure, as it would add to the length of administration 
of the measure. However, it does bring out the need for patient's perspective on the items administered in the test. The way this concept was incorporated in the test is having the patient/client choose one item from the measure that provided them just right challenge and they felt was important to them to track their progress in therapy.

Conditions. The experts noted that the hand function deficits do differ with the diagnosis and some conditions like Parkinson disease could have specific symptoms like tremors and medication related effects that should be considered if the test is to be valid for use in people with Parkinson disease. There was a discussion about external cues needed for people with PD during intervention, the issues related to progression of the disease and presence of ataxic arm movements. The more frequency of excerpts was found for the three PD symptoms of tremors, slow movements and rigidity and these were added as symptoms to the self-report measure. The issue of good function on medication (also known as 'on - phase') and poor function off medication (also known as 'off - phase') is specific to PD.

So external cues might be specific to Parkinson's, even on and off medications, it's a huge difference. It is necessary to know how long it has been since their last dose of medication. (E4)

The details about medications were included in the patient/client information section of both self-report and performance-based sections of the measure.

For stroke, experts had comments about learned non-use, hand preference, tone, using the strong hand to help the weak hand, and problems with releasing objects.

And also for stroke you might be changing your hand preference. (E1)

The change in hand function was captured in patient/client information section with question about current and prior hand preference. Tone was added in the symptom list as spasticity and 
rigidity symptoms. For multiple sclerosis, the experts agreed that it presented with a varied range of symptoms that are mostly bilateral. For TBI, the experts mentioned that there were more cognitive deficits, bilateral involvement and may not have the presentation of hemiplegia. The idea of providing more cues during testing was discussed in relation to TBI and it was suggested that although cues would be needed for some patients they do serve as a form of assistance and should be treated accordingly in the measure.

Breaking it down into smaller steps. But again that problem with simulated versus real world comes in right, if you always have the test incorporate a cue like put the pen on a shelf, they may not have a shelf in their life, they may have to just lift something up. They may not have an external cue all the time. (E3)

People with neurological conditions have other associated orthopedic issues.

So, my mother had rheumatoid arthritis and I think about that. (E2)

It seemed important to have people list all their diagnoses in the patient/client information section at the beginning of the test for the therapist to have information about other diagnoses that could affect their hand function.

Items. Fifty-six items were suggested by experts from experience with patients with neurological conditions. These items were added to the item bank to be combined with items suggested by patient/client participants in focus groups for a total set of 144 items in the item bank. A subset of the items in the item bank were to be administered to patient/client participants for cognitive interviews and a method for item extraction was needed. For the purpose of item development, a strategy for triangulation of results from expert participants and patient/client participants was developed. The frequency of occurrence of each item among patients and experts was combined with the frequency of co-occurrence of each item for all groups. The items 
were then ranked by their total occurrence score and top one-third of the items that had total occurrence score of 6 or higher were selected for further development. Items were later added based on theoretical constructs to be addressed.

Paper and pencil, puzzle, spoon and fork, something that most people have a wallet full of credit cards, money and some change, I think that would give a lot of fine motor information and you don't necessarily have to carry it, you can use the patient's regular wallet or whatever, that seems to be a good one. Keys, set of keys, a zipper of some sort, buttons, or so these are irrespective of gender, irrespective of diagnoses, these could be some of the items. (E3)

The high frequency items suggested by expert participants were using a electronic tablets (such as iPad), self care in general, eating, using a spoon or fork, dressing, hygiene, using a pen and handling coins. Technology related items are not seen in the hand function tests and yet are part of the daily repertoire of activities that people engage in. Self-care, eating and using utensils are generic tasks that the experts agreed needed to be included in a measure that targets daily activities. Dressing and hygiene is general were also reported as important elements of daily activities. The distinction between self-care and hygiene was not clear and thus the excerpts were coded by the term with which the expert referred to the task.

Hand function. The experts were able to weigh in on aspects of hand function that should be considered when designing the items.

I always ask, which hand are they using, what's their dominance like, what their sensation like. (E3)

They discussed fine motor skills such as manipulation including in-hand manipulation, coordination, speed, performance efficiency, accuracy, tone, sensation including proprioception, 
range of motion, grip, grasp, pinch, release, and turning. They discussed gross motor skills such as reaching, lifting, bimanual function, arm swing when walking, and prior level of function. They also indicated specific problems such as learned non-use and pain in stroke, tremors and problems with rapid alternating hand movements in PD, neglect of one side, and fatigue with MS. They discussed theories that can affect assessment such as motor control, modulation of force, motor planning, and dual tasking.

Test. Expert participants were actively involved in the discussion regarding hand function testing. They cited examples of other tests that they have used in the clinic that included the Jebsen Hand Function Test, the Box and Block Test, the dynamometer, the Wolf Motor Function Test, the Unified Parkinson's Disability Rating Scale, the Comprehensive Occupational Performance Measure, and the Disabilities of the Arm Shoulder and Hand. Pediatric tests were also mentioned as one expert had experience working with children as well. The items from these measure might have influenced the perspectives of the expert participants however they were discouraged to use items directly from these measures in keeping with fair use of the commercially available tests. No items in this measure were taken from any other measures of hand function.

They also discussed the way they were currently testing patients. Ya depends on why you were coming, why you were referred. I might while I am talking about what we are going to do, I might start moving your arm feeling range of motion and pain while we are chatting so I have kind of a clue, that what I am looking. Then I might do a thing like I might ask you to reach overhead, that kind of quick and dirty. (E1) 
They emphasized the fact that the measure would be useful if it could be quickly administered. The experts agreed that speed of task was important but quality of the task was equally important.

If it takes too long its not going to be functional. If it takes you 40 minutes to get your shirt your shirt on, you are not going to do it. ..maybe what they are doing that you are concerned about is kind of unsafe, the way that they are leaning over to get their shirt on they are going to fall off the bed and its unsafe for them.

But because if you saw them in 6 months they cannot do that compensation anymore well enough so now they cannot do it. (E1)

They also noted that quality was important for progressive conditions like PD or multiple sclerosis as well. The measurement expert expressed the need for a variety of items that would tap into function (e.g. grasp) and function issues or problems with function (e.g. tone).

Okay, so from a measurement perspective you would want to have a variety of different tasks that we will do really tap into the different functions. (E2)

There were other comments about giving the patients a choice of the activity they want to choose for were noted but not implemented for actual testing. However, it was noted that the individuals will get a choice of which task of the test they want to track over time that can give them information that might be meaningful to them.

The experts also expressed the need for the self-report section to come before the performancebased section.

I think the questions come first. I think, you have this patient. You find out why they are there, what is the problem. (E1) 
The experts also expressed a need for individualized item to track progress.

In my opinion I think for me it's so heavily guided by what the person tells me in their history that its difficult to generalize sometimes. I mean the general things we have talked about like reaching, grasping, in-hand manipulation, strength, sensation, those are like the basic things that I think we look for in everyone. But the rest of the finer things are so heavily guided by what they like to do you know, were they golfing before, were they flyfishing before. So then its heavily guided by individual interests and what they are used to doing. (E4)

Since, this could not be done in a performance-based assessment (to maintain standardized format for consistency of testing), it was decided to incorporate this in the self-report section. Moreover, since individual interests play out in higher level tasks such as work, school, and leisure, it was logical to provide an individualized item for these categories. This was built into the measure.

The experts also expressed a desire for the measure to have low equipment needs, have a standardized protocol, be able to track progress, familiar tasks, use across diagnosis, and incorporate universal instructions. The two domains of gross motor and fine motor were identified by experts and keeping a standard expectation of patient/client able to maintain sitting balance or be ambulatory needs to be set up. The experts also identified problems with current tests such as taking more time, not providing much added information, cost money, cannot find pieces of test equipment or directions that are needed, require a lot of set up time, lack gross motor skill measurement, need to guess function from scores, and the presence of too many items. 
I don't know, but I would say that the big problem I see with these standardized tests for me is they take more time, and I don't get any more information than I got before. And they sometimes cost a lot of money and you cannot find the piece that you needed. You know. (E4)

Or the directions. (E3)

Or you have just an hour and I am spending 30 minutes on this test, setting it up. (E4)

Experts also agreed that the patients should be given time to understand the item, but that they may not need as much time the next time you administer the item. They agreed with giving cues or practice trials or written directions if needed. When asked about scores, there were indications that scores would be used to guide intervention but there were also strong feelings about scores needed to support documentation requirements for insurances.

I don't necessarily think practitioners use the scores, they look at what is happening and act on what is happening, it's the insurance companies who want the scores. (E2)

The other bodily functions that that affect performance on the Hand and Arm Function Measure were discussed by the group and included visual perception, visual acuity, balance, cognition, motor planning, standing, changed dominance, walking, and exercise. The measure would have to be designed to reduced variance caused by these factors on arm and hand function.

The item writing and modification phase was planned after patient/client focus groups. This was done to incorporate patient/client perspectives in the development of the construct and items before item writing. 


\section{Discussion and Conclusion}

The expert panel focus group was able to provide insights into the deficiencies in existing measures, purpose of the new measure, the measurement criteria, the items that would need to go into the measure, factors that would affect hand and arm function. They were able to provide insight into conceptual understanding of hand use versus function, simulated versus real life tasks, and compensation for functional deficits that can affect measurement of hand and arm function. This expert panel study and its systematic investigation of the opinions of therapists in a qualitative research format contributes important information to the body of literature on hand and arm function related to assessment.

There is a need for development of measures that are valid in neurological conditions (Kraft et al., 2014). However, no studies have utilized the qualitative methodology to formally interview therapists and measurement experts to inform test development. Some existing measures (i.e., Disabilities of Arm Shoulder and Hand) that have utilized reviews by panels of experts (Hudak et al., 1996), have done so without explicitly documenting the results of their involvement. Some other existing measures have been developed by occupational therapists and item development has been done based on other measures or psychometric analysis (Chen et al., 2005) without involving other disciplines such as physical therapists or measurement specialists who have experience with a specific diagnostic population.

Such expert panel members are referred to as 'subject matter experts' or 'content evaluation experts, by some authors in psychology (Lawshe, 1975). The experts were all women, which is representative of the make up of the field of physical and occupational therapy (American Occupational Therapy Association, 2010; Washington University in St. Louis, 2009). 
There is a chance that the lack of inclusion of male experts however could create a bias in the items suggested and perspectives regarding daily activities.

Stakeholders are the individuals impacted by the use or effects of the measurement process. Since therapists are the primary individuals who will be administering this measure, involving these stakeholders early on in the construct development phase will provide evidence towards the face and content-related evidence for validity of the scores of the measure. The use and interpretation of test scores by stakeholders constitutes aspects of the construct-related evidence for validity of the scores of the measure (Messick, 1994). The expert panel input in this development phase ensures that the scores are intended to be used for the purpose outlined by the stakeholders. 
Table 3.1. Construct Scheme to Represent Hand and Arm Function Dimensions and Items.

\begin{tabular}{|c|c|c|c|c|}
\hline$I C F$ & Dimensions & Hand Function & $\begin{array}{l}\text { Self-report items of Hand } \\
\text { and Arm Function Measure }\end{array}$ & $\begin{array}{l}\text { Performance-based items } \\
\text { of Hand and Arm Function } \\
\text { Measure }\end{array}$ \\
\hline \multirow{22}{*}{$\begin{array}{l}\text { Activity and } \\
\text { Participation }\end{array}$} & \multirow{6}{*}{$\begin{array}{l}\text { Gross movements in daily } \\
\text { activities }\end{array}$} & \multirow[t]{2}{*}{ Transport/ reach } & Toilet hygiene & Hand to lower back \\
\hline & & & $\begin{array}{l}\text { Washing hair } \\
\text { Getting dressed }\end{array}$ & $\begin{array}{l}\text { Hand to back of head } \\
\text { Grabbing jar at shoulder } \\
\text { height }\end{array}$ \\
\hline & & \multirow{4}{*}{$\begin{array}{l}\text { Non -prehensile skilled } \\
\text { movements (pushing, } \\
\text { carrying) }\end{array}$} & Clap hands & $\begin{array}{l}\text { Bean can task - grasping } \\
\text { and carrying }\end{array}$ \\
\hline & & & $\begin{array}{l}\text { Shaking hands when } \\
\text { greeting }\end{array}$ & \\
\hline & & & Holding an open book & \\
\hline & & & Lifting grocery bag & \\
\hline & \multirow[t]{16}{*}{$\begin{array}{l}\text { Fine movements in daily } \\
\text { activities }\end{array}$} & \multirow[t]{12}{*}{$\begin{array}{l}\text { Prehension - Grip \& } \\
\text { pinch }\end{array}$} & $\begin{array}{l}\text { Lifting gallon of milk } \\
\text { Lift cup with liquid in it }\end{array}$ & Coffee mug \\
\hline & & & Open door lock with key & Open lock with key \\
\hline & & & Open \& close jar & Open jar \\
\hline & & & Feeding yourself & Spoon use \\
\hline & & & Cutting finger nails & Address writing \\
\hline & & & Items in \& out of wallet & $\begin{array}{l}\text { Pennies in bowl - pick \& } \\
\text { place }\end{array}$ \\
\hline & & & Clothing fasteners & Peas in bottle \\
\hline & & & Open can & \\
\hline & & & Brushing teeth & \\
\hline & & & Putting on watch or jewelry & \\
\hline & & & Cutting vegetables & \\
\hline & & & Opening milk carton & \\
\hline & & In-hand manipulation & $\begin{array}{l}\text { Handling credit cards or } \\
\text { money }\end{array}$ & $\begin{array}{l}\text { Pennies in bowl - pick \& } \\
\text { hold }\end{array}$ \\
\hline & & $\begin{array}{l}\text { Non -prehensile skilled } \\
\text { movements (pointing, } \\
\text { turning) }\end{array}$ & $\begin{array}{l}\text { Open \& close Ziploc® bag } \\
\text { Turning door knob } \\
\text { Press buttons on phone or } \\
\text { remote control, keyboard } \\
\text { Computer mouse }\end{array}$ & $\begin{array}{l}\text { Open lock with key, open } \\
\text { spice bottle }\end{array}$ \\
\hline & & Active haptic mode & $\begin{array}{l}\text { Using a touch screen on a } \\
\text { phone, tablet, computer, } \\
\text { laptop }\end{array}$ & Sensory test - stereognosis \\
\hline & & Passive tactile sensing & $\begin{array}{l}\text { Sensation loss } \\
\text { Hypersensitivity }\end{array}$ & \\
\hline $\begin{array}{l}\text { Body Function/ } \\
\text { Structure }\end{array}$ & $\begin{array}{l}\text { Symptoms interfering } \\
\text { with hand function in } \\
\text { daily activities }\end{array}$ & $\begin{array}{l}\text { Low functioning: Non- } \\
\text { movement related } \\
\text { aspects }\end{array}$ & $\begin{array}{l}\text { Pain } \\
\text { Spasticity } \\
\text { Tremor } \\
\text { Slow movements } \\
\text { Deformity } \\
\text { Stiffness } \\
\text { Weakness } \\
\text { Coordination } \\
\text { Sleep } \\
\text { Fatigue }\end{array}$ & \\
\hline Participation & Occupational roles & $\begin{array}{l}\text { High functioning: High } \\
\text { demand for productive } \\
\text { function }\end{array}$ & $\begin{array}{l}\text { Work } \\
\text { Childcare } \\
\text { School } \\
\text { Leisure } \\
\end{array}$ & \\
\hline
\end{tabular}


Table 3.2. Design Pattern Attributes with Corresponding Messick and Toulmin Argument Components and Application to Hand and Arm Function Measure.

\begin{tabular}{|c|c|c|c|}
\hline Design Pattern Attribute & $\begin{array}{l}\text { Messick } \\
\text { Assessment } \\
\text { Argument } \\
\text { Component }\end{array}$ & $\begin{array}{l}\text { Toulmin } \\
\text { Assessment } \\
\text { Argument } \\
\text { Component }\end{array}$ & $\begin{array}{l}\text { Application to Hand and Arm Function } \\
\text { Measure }\end{array}$ \\
\hline Rationale & $\begin{array}{l}\text { Student Model/ } \\
\text { Claim: What } \\
\text { construct should } \\
\text { be assessed? }\end{array}$ & Warrant & $\begin{array}{l}\text { Study of available measures in the systematic } \\
\text { review, ICF framework, NIH focus on } \\
\text { patient/client reported outcomes and NIH } \\
\text { toolbox efforts to support similar developments } \\
\text { in the field. }\end{array}$ \\
\hline $\begin{array}{l}\text { Knowledge, skills and } \\
\text { abilities }\end{array}$ & & Claim & $\begin{array}{l}\text { Hand and arm function can be measured with a } \\
\text { combination of: 1) Clinician's observation of a } \\
\text { patient/client performing timed non-prehensile } \\
\text { and prehensile tasks, and 2) Self-reported rating } \\
\text { of symptoms and daily activity tasks related to } \\
\text { hand and arm function. }\end{array}$ \\
\hline $\begin{array}{l}\text { Additional knowledge, } \\
\text { skills and abilities }\end{array}$ & & $\begin{array}{l}\text { Claim/Alternative } \\
\text { Explanation }\end{array}$ & $\begin{array}{l}\text { 1) Personal factors 2) Body structure factors } 3 \text { ) } \\
\text { Cognition and other body function factors 4) } \\
\text { Environmental factors }\end{array}$ \\
\hline Potential work products & $\begin{array}{l}\text { Evidence } \\
\text { Model/Actions: } \\
\text { What behaviors } \\
\text { should reveal } \\
\text { the construct? }\end{array}$ & $\begin{array}{l}\text { Data about } \\
\text { student } \\
\text { (patient/client) } \\
\text { performance }\end{array}$ & $\begin{array}{l}\text { Performance section: Patient/client will } \\
\text { participate in tasks that will be scored on a } \\
\text { rubric to generate scores } \\
\text { Self-report section: Patient/client will mark or } \\
\text { indicate on the questionnaire interference of } \\
\text { hand and arm function issues in daily tasks. }\end{array}$ \\
\hline Potential observations & & & $\begin{array}{l}\text { Performance section: Time, assistance, } \\
\text { compensation and completion are observed by } \\
\text { a skilled observer and recorded in the scoring } \\
\text { rubric. } \\
\text { Self -report section: Interference of hand and } \\
\text { arm function issues in daily tasks and } \\
\text { interference of symptoms on hand and arm } \\
\text { function will indicate the functional level of a } \\
\text { person. }\end{array}$ \\
\hline $\begin{array}{l}\text { Characteristic task } \\
\text { features }\end{array}$ & $\begin{array}{l}\text { Task } \\
\text { Model/Situation: } \\
\text { What tasks } \\
\text { should elicit } \\
\text { those } \\
\text { behaviors? }\end{array}$ & $\begin{array}{l}\text { Data about } \\
\text { assessment } \\
\text { context/situation }\end{array}$ & $\begin{array}{l}\text { Performance section: The daily activity task } \\
\text { features are broadly divided into gross and fine } \\
\text { movement dimensions. The aspects of } \\
\text { sensation, strength, grasping, and fine hand use } \\
\text { aspects were considered based on expert and } \\
\text { patient/client feedback. } \\
\text { Self-report section: The task features are } \\
\text { broadly divided into ICF categories of body } \\
\text { structure and function, activity and } \\
\text { participation. Within the activity and } \\
\text { participation items related to daily tasks, the } \\
\text { tasks were divided into self-care, kitchen, } \\
\text { technology, miscellaneous, work and leisure. }\end{array}$ \\
\hline Variable task features & & & $\begin{array}{l}\text { There is a range of complexity of tasks that a } \\
\text { person can engage in that will give information } \\
\text { about low to high trait of hand and arm function } \\
\text { is demonstrated. }\end{array}$ \\
\hline
\end{tabular}


Table 3.3. Comparison of Original and Revised Construct Definition, Impact of Revision on Assessment Design, and Studies to Support Evidence for Validity of Scores

\begin{tabular}{|c|c|c|c|}
\hline $\begin{array}{l}\text { Original } \\
\text { Construct } \\
\text { definition }\end{array}$ & Revised Construct definition & $\begin{array}{l}\text { Impact of revision on } \\
\text { assessment design }\end{array}$ & $\begin{array}{l}\text { Impact of revision on studies to } \\
\text { support validity of scores }\end{array}$ \\
\hline $\begin{array}{l}\text { Measures hand use } \\
\text { with fine motor } \\
\text { tasks }\end{array}$ & $\begin{array}{l}\text { Measures hand and arm function - } \\
\text { Broader outlook includes gross } \\
\text { and fine movement domains. }\end{array}$ & $\begin{array}{l}\text { Reduces construct under- } \\
\text { representation, develop } \\
\text { arguments with a broader } \\
\text { definition }\end{array}$ & $\begin{array}{l}\text { Includes individuals with high and } \\
\text { low levels of the trait of hand and arm } \\
\text { function. Extends the use arguments } \\
\text { due to more coverage of the range of } \\
\text { impairments. }\end{array}$ \\
\hline $\begin{array}{l}\text { Includes one } \\
\text { dimension - fine } \\
\text { motor tasks }\end{array}$ & $\begin{array}{l}\text { Includes two correlated } \\
\text { dimensions in performance-based } \\
\text { section, i.e., fine motor and gross } \\
\text { motor; two correlated dimensions } \\
\text { in self-report section, i.e., body } \\
\text { structure and function and activity } \\
\text { and participation }\end{array}$ & $\begin{array}{l}\text { Task model would include } \\
\text { fine and gross movements, } \\
\text { symptoms, daily tasks and } \\
\text { become more robust, } \\
\text { reduces construct irrelevant } \\
\text { variance }\end{array}$ & $\begin{array}{l}\text { Concurrent evidence supported by } \\
\text { correlations between the measure and } \\
\text { other hand function measures for both } \\
\text { performance-based and self-report } \\
\text { sections will need to be expanded. }\end{array}$ \\
\hline $\begin{array}{l}\text { Sub domains for } \\
\text { items } \\
\text { Control: Strength, } \\
\text { stability, mobility, } \\
\text { pain and } \\
\text { coordination. }\end{array}$ & $\begin{array}{l}\text { Sub-domains defined in each } \\
\text { dimension using Jones and } \\
\text { Lederman's framework: } \\
\text { Transport, reach, prehensile, non- } \\
\text { prehensile, passive sensation, } \\
\text { active haptic mode. Low and high } \\
\text { functioning sub-domains added. }\end{array}$ & $\begin{array}{l}\text { Reduces construct under- } \\
\text { representation, arguments } \\
\text { need to develop for } \\
\text { interpretation, use, value and } \\
\text { consequences }\end{array}$ & $\begin{array}{l}\text { Discriminant evidence will need to be } \\
\text { established by selecting measures that } \\
\text { are truly different considering that the } \\
\text { definition of the construct is now } \\
\text { wider. }\end{array}$ \\
\hline $\begin{array}{l}\text { Includes tasks (not } \\
\text { clearly defined) }\end{array}$ & Includes daily life tasks & $\begin{array}{l}\text { Impacts the use argument } \\
\text { and consequences }\end{array}$ & $\begin{array}{l}\text { Predictive evidence for outcomes } \\
\text { predicted by this measure will need to } \\
\text { be selected based on the daily-activity } \\
\text { nature of the tasks. }\end{array}$ \\
\hline $\begin{array}{l}\text { Diagnoses not } \\
\text { specified }\end{array}$ & $\begin{array}{l}\text { Includes neurological conditions } \\
\text { with diagnostic groups specified. }\end{array}$ & $\begin{array}{l}\text { Impacts generalizability, use } \\
\text { argument and consequences }\end{array}$ & $\begin{array}{l}\text { Population selected for studies will be } \\
\text { specific to only the specified } \\
\text { neurological conditions. } \\
\text { Generalization of test results to only } \\
\text { those diagnoses is reasonable. }\end{array}$ \\
\hline $\begin{array}{l}\text { Response process } \\
-5 \text { choices with } \\
\text { 'don't do' option }\end{array}$ & $\begin{array}{l}\text { Response process }-3 \text { choices in } \\
\text { self-report and } 5 \text { choices in } \\
\text { performance-based. Scoring to } \\
\text { exclude missing items. }\end{array}$ & $\begin{array}{l}\text { Improved response process } \\
\text { Reduces construct irrelevant } \\
\text { variance }\end{array}$ & $\begin{array}{l}\text { Selection of scoring model can } \\
\text { impact statistical analysis. }\end{array}$ \\
\hline $\begin{array}{l}\text { Adults with hand } \\
\text { function problems }\end{array}$ & $\begin{array}{l}\text { Adults - includes male and } \\
\text { female, all cultures, rural and } \\
\text { urban setting, inpatient and } \\
\text { outpatient settings. }\end{array}$ & $\begin{array}{l}\text { Improved validation of } \\
\text { interpretations to the varied } \\
\text { subgroups }\end{array}$ & $\begin{array}{l}\text { Sample of individuals to be included } \\
\text { in studies needs to be stratified based } \\
\text { on gender, culture \& settings. }\end{array}$ \\
\hline $\begin{array}{l}\text { Consequences - } \\
\text { not developed }\end{array}$ & $\begin{array}{l}\text { Consequences - Intended: } \\
\text { Intervention outcomes, determine } \\
\text { level of impairment, need for } \\
\text { services. } \\
\text { Unintended: Patient or therapist's } \\
\text { perceptions of level of impairment } \\
\text { or outcomes }\end{array}$ & $\begin{array}{l}\text { Claims to be made about the } \\
\text { patient/client will be } \\
\text { impacted. }\end{array}$ & $\begin{array}{l}\text { For intervention - minimal clinically } \\
\text { important difference will need to be } \\
\text { estimated. For level of impairment - } \\
\text { cut off scores will need to be } \\
\text { established. For need for services - } \\
\text { data related to qualification for } \\
\text { services will need to be compared. }\end{array}$ \\
\hline
\end{tabular}


Table 3.4. Interview Guide for Expert Panel Focus Group.

Semi-structured questions: Experts

About the construct

- What came to your mind when I said arm and hand use?

- What if I would have said arm and hand function? What would that mean to you?

- Can you tell me what is important to measure about arm and hand use? Why? How?

- Do you remember any tests of arm and hand function that you might have encountered in the past? Can you tell me about them? What were the aspects that you liked or did not like about them?

- Do you think, it would make sense to have a test of arm and hand use include both a questionnaire and some timed activities? Why or why not?

- In what form would you like to inform the results from a test of arm and hand use?

About domains of hand function

- Can you tell me what are some of important aspects or domains of hand function?

- Can you talk about how we can gather information about these domains using questions about daily tasks?

- Can you talk about how we can gather information about these domains using time performance tasks?

- What about arm and hand use in standing tasks? Does it matter?

- What about sensation?

About specific neurological conditions

- Now we will shift gears and talk about specific neurological condition and how that affects arm and hand use.

- Stroke and arm/hand use?

- Traumatic brain injury and arm/hand use?

- Multiple Sclerosis and arm/hand use?

- Parkinson Disease and arm/hand use?

About items on a test

- A test cannot have too many items. What are some of the daily activities that might be very important to measure?

- Do we need to ask about bilateral activities?

- Do we need to ask about dominance? Before or after the diagnosis?

- What about pain?

- What about fatigue?

Additional questions and discussion may follow based on the level of detail provided by each group member. 
Table 3.5. Demographics of Experts

\begin{tabular}{|c|c|c|c|c|c|c|}
\hline Expert & $\begin{array}{l}\text { Degree and } \\
\text { Profession }\end{array}$ & $\begin{array}{l}\text { Clinical } \\
\text { Practice }\end{array}$ & $\begin{array}{l}\text { Clinical } \\
\text { Experience }\end{array}$ & $\begin{array}{l}\text { Research } \\
\text { Experience }\end{array}$ & $\begin{array}{l}\text { Common Neurological } \\
\text { Conditions in Clinic }\end{array}$ & $\begin{array}{l}\text { Other related } \\
\text { Information }\end{array}$ \\
\hline E1 & $\begin{array}{l}\text { MS, } \\
\text { Occupational } \\
\text { Therapy }\end{array}$ & $\begin{array}{l}\text { Active in } \\
\text { pediatrics }\end{array}$ & 40 years & N/A & $\begin{array}{l}\text { Brain tumors, brachial } \\
\text { plexus injury, cerebral } \\
\text { palsy, epilepsy, } \\
\text { peripheral nerve injury, } \\
\text { polyneuropathy, stroke, } \\
\text { and traumatic brain } \\
\text { injury }\end{array}$ & $\begin{array}{l}\text { Teaching } \\
\text { experience }\end{array}$ \\
\hline E2 & $\begin{array}{l}\mathrm{PhD}, \\
\text { Measurement }\end{array}$ & N/A & N/A & 30 years & $\mathrm{N} / \mathrm{A}$ & $\begin{array}{l}\text { Teaching } \\
\text { experience } \\
\text { Published } \\
\text { research related } \\
\text { to measurement } \\
\text { in rehabilitation }\end{array}$ \\
\hline E3 & $\begin{array}{l}\text { BS, } \\
\text { Occupational } \\
\text { Therapy }\end{array}$ & $\begin{array}{l}\text { Active in } \\
\text { adult care - } \\
\text { neuro- } \\
\text { rehabilitation } \\
\text { out-patient }\end{array}$ & 30 years & N/A & $\begin{array}{l}\text { Brachial plexus injury, } \\
\text { dementia, essential } \\
\text { tremor, Guillian Barre } \\
\text { Syndrome, multiple } \\
\text { sclerosis, Parkinson } \\
\text { Disease, stroke, and } \\
\text { traumatic brain injury }\end{array}$ & N/A \\
\hline E4 & $\begin{array}{l}\text { PhD, Physical } \\
\text { Therapy }\end{array}$ & $\begin{array}{l}\text { Active in } \\
\text { skilled nursing } \\
\text { facility }\end{array}$ & 11 years & 7 years & $\begin{array}{l}\text { Brain tumors, } \\
\text { dementia, multiple } \\
\text { sclerosis, Parkinson } \\
\text { disease, stroke, and } \\
\text { traumatic brain injury }\end{array}$ & $\begin{array}{l}\text { Teaching } \\
\text { experience } \\
\text { Published } \\
\text { research related } \\
\text { to measurement } \\
\text { in rehabilitation }\end{array}$ \\
\hline
\end{tabular}




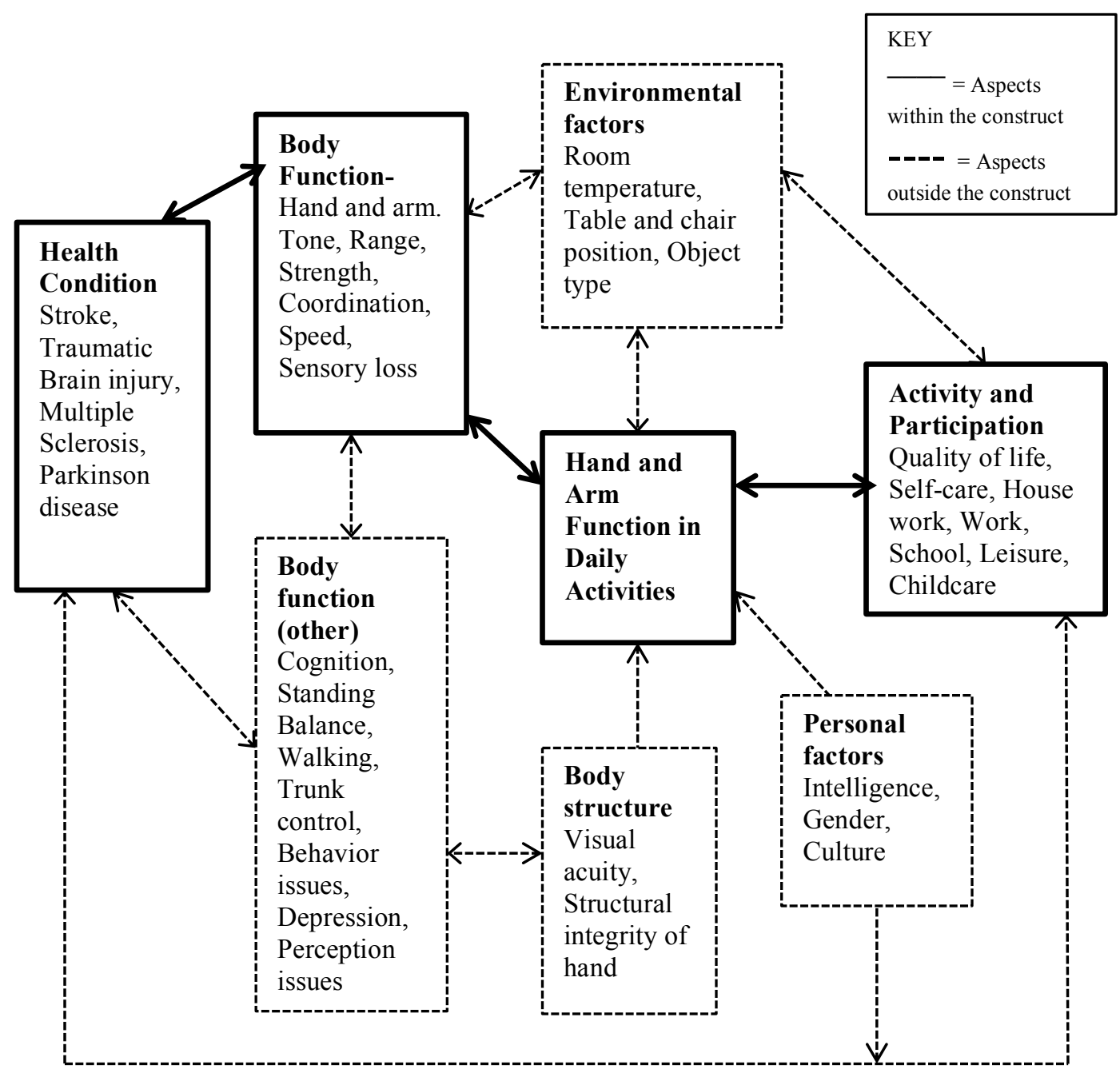

Figure 3.1. Nomological Network for Hand and Arm Function Measure 


Claim about patient/
client

High level of the trait of hand and arm function
Hand and arm

function

\begin{tabular}{|c|c|c|}
\hline $\begin{array}{l}\text { Individual with good hand function for } \\
\text { participation in daily life tasks }\end{array}$ & $\uparrow$ & $\begin{array}{l}\text { High scores on performance and self- } \\
\text { report in both dimensions of fine and } \\
\text { gross movements }\end{array}$ \\
\hline $\begin{array}{l}\text { Individual with mild difficulty with } \\
\text { hand use in daily life tasks }\end{array}$ & & $\begin{array}{l}\text { Moderate scores on performance and } \\
\text { self-report in both dimensions of fine } \\
\text { and gross movements }\end{array}$ \\
\hline $\begin{array}{l}\text { Individual with moderate difficulty } \\
\text { with hand use in daily life tasks }\end{array}$ & & $\begin{array}{l}\text { Low scores on performance and self- } \\
\text { report in both dimensions of fine and } \\
\text { gross movements }\end{array}$ \\
\hline $\begin{array}{l}\text { Individual with severe difficulty with } \\
\text { hand use in daily life tasks }\end{array}$ & $\downarrow$ & $\begin{array}{l}\text { Very low scores on performance and } \\
\text { self-report in both dimensions of fine } \\
\text { and gross movements }\end{array}$ \\
\hline
\end{tabular}

Low level of the trait of hand and arm function

Figure 3.2. Construct Map for Hand and Arm Function Measure 


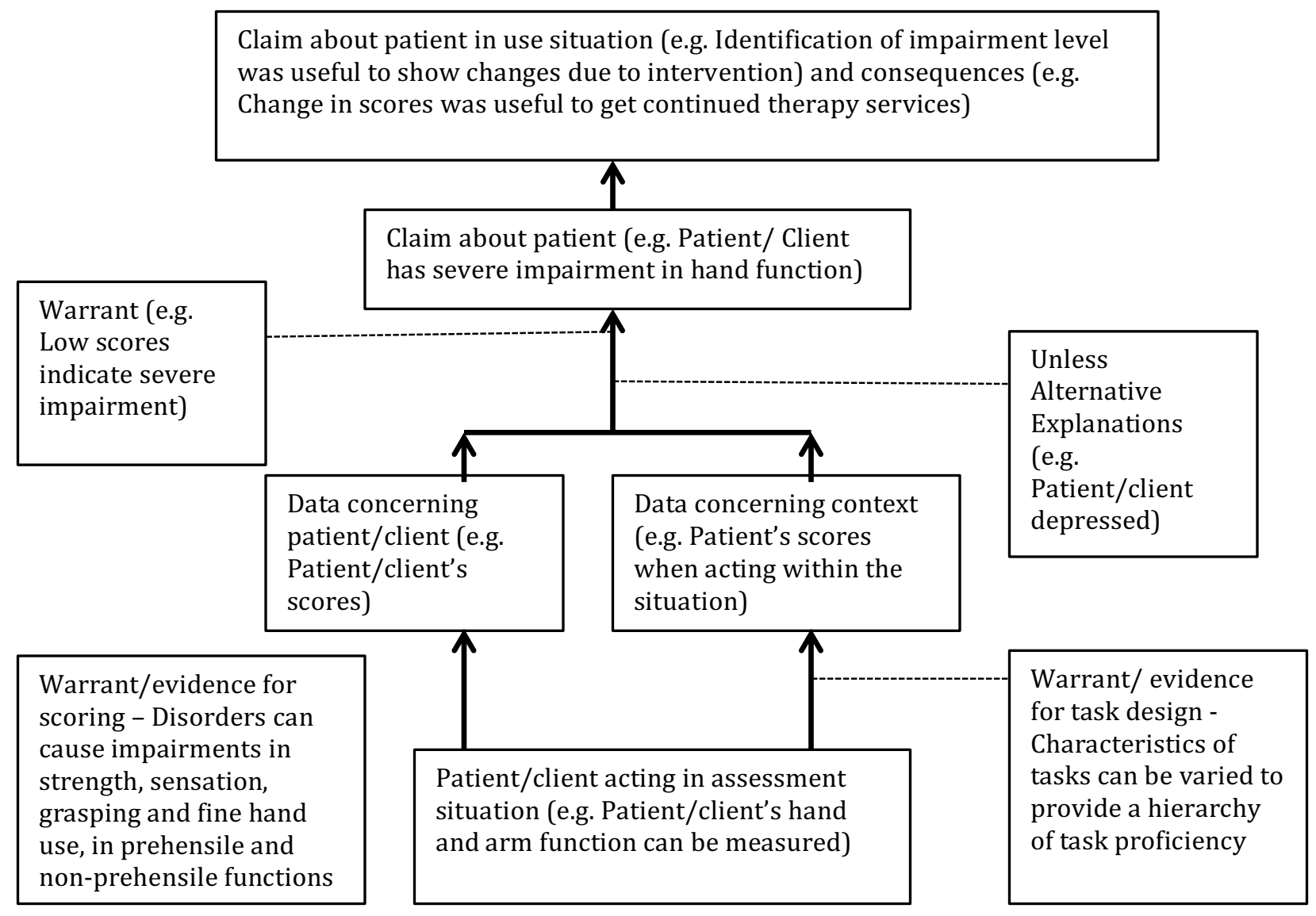

Figure 3.3. Toulmin Diagram for Hand and Arm Function Measure 


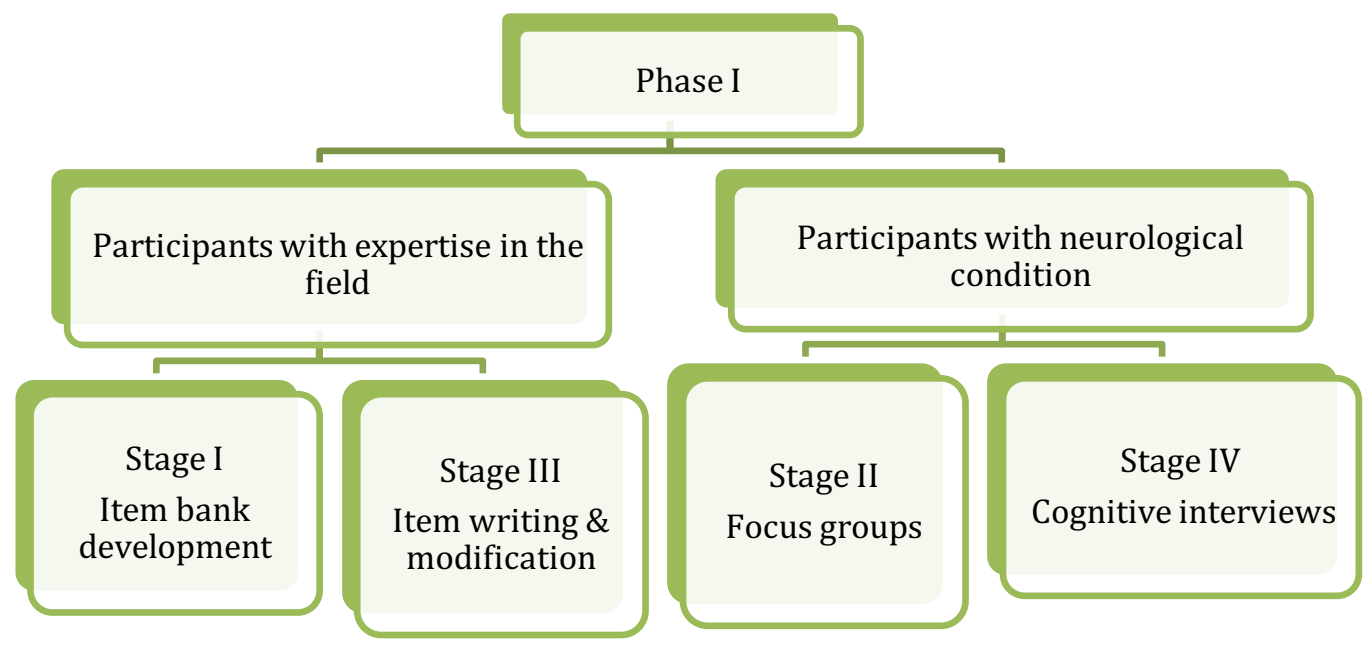

Figure 3.4. Representation of the Four Stages in Research Study 


\title{
Chapter 4: Focus Groups and Cognitive Interviews Study
}

\begin{abstract}
Background and purpose: A new measure of hand and arm function in daily activities is being developed for use in people with neurological conditions. For the measure to accurately reflect the difficulties experienced by people with neurological conditions, a qualitative study was designed to gain their perspectives. No study currently exists that explores the experiences of people with neurological conditions in relation to their hand and arm function in daily activities.
\end{abstract}

Objective: This study aimed to explore the views of adults with neurological conditions regarding hand and arm function in daily activities and their opinion about a preliminary set of items to develop a new measure.

Method and Results: A descriptive qualitative approach involved seven focus groups and eight cognitive interviews. Twenty participants were recruited among four diagnostic conditions with purposeful sampling and included seven people with stroke, two with traumatic brain injury, six with Parkinson disease, and five with multiple sclerosis. All interviews were transcribed verbatim and thematically analyzed. The focus group discussion helped identify aspects of hand function that were relevant to the participants with neurological condition such as sensation, strength, grasping, and fine hand use. The feedback from focus group participants helped develop the item bank. The item bank from participants with neurological condition was then pooled with the items from expert panel study previously conducted to identify the high frequency items to be included in the preliminary set administered to the participants in the form of one-on-one cognitive interviews. The cognitive interview study found that self-reported and performance-based aspects of function were well received by the participants. The self-report 
items needed definitions of the terms included in the questions, an additional response option and a comment box, and change in the order of presentation of items with bimanual followed by unimanual items. The performance-based items needed to capture difficulties on the affected side along with preserving the bimanual nature of the tasks.

Conclusions: The focus groups and cognitive interviews facilitated the grounding of the measure in the views of participants with neurological conditions. Thus contributing to the face and content-related evidence for validity of scores of the new measure. 


\section{Introduction}

A new measure of upper extremity function in daily activities - Hand and Arm Function Measure (HAFM) - is being developed for use in people with neurological conditions. For the measure to accurately reflect the difficulties experienced by people with neurological conditions, a qualitative study was designed to seek their views. No study that explores the experiences of people with neurological conditions in relation to their hand and arm function in daily activities was found in the current literature.

This study was part of the first phase of the broad research project for development of HAFM. The first phase consisted of four stages; i.e., I) item bank development with expert panel focus group discussions, II) focus group discussions with participants with neurological condition, III) item writing and modification, and IV) cognitive interviews with participants with neurological conditions. The participants with neurological condition were involved in Stage II and IV.

\section{Purpose}

The objective of this study was to explore the views of adults with neurological conditions regarding hand and arm function in daily activities to develop an item bank and to gather their opinion about a preliminary set of items to measure hand and arm function. The broader objective of this research is to develop a new measure of hand and arm function.

\section{Method}

Qualitative descriptive research methodology of focus groups and face-to-face semistructured interviews were used to obtain in-depth data from participants with neurological conditions. These methods were selected to facilitate a group discussion, gather ideas about potential items through group validation, identify commonly used terms for the medical 
condition, and identify perceptions regarding measurement of hand and arm function. The neurological conditions of interest were stroke, traumatic brain injury (TBI), multiple sclerosis (MS), and Parkinson disease (PD). These diagnostic categories were selected to represent progressive and non-progressive neurological conditions. Over a lifespan, MS and PD have a progressive course and stroke and TBI have a non-progressive course of development. These are the diagnostic categories most commonly encountered by therapists in the rehabilitation setting. Approval from the University of Washington Human Subjects Division was obtained prior to commencing the study.

Participants. The participants were recruited with flyers posted at University of Washington clinics (Seattle, WA), Evergreen Health clinics (Kirkland, WA), research participant recruitment websites, and contacting therapists in the community to inform about this study. Also research study information was posted in newsletters for registries available through various organizations such as University of Washington Traumatic Brain Injury Model System.

The purposeful sampling of participants was done with the following inclusion and exclusion criteria. The inclusion criteria were participants: 21 years of age or older, overall good health, mild, moderate or severe difficulty using one's arm or hand that interfered with daily activities, had been diagnosed by a physician to have either stroke, traumatic brain injury, Parkinson disease, or multiple sclerosis, diagnosed at least 6 months or prior, and able to arrange travel to the University of Washington for the research study and were an English language speaker. The exclusion criteria were: concurrent other neurological condition(s) that would affect arm or hand function, currently receiving physical or occupational therapy, limb loss or any injury to the arm or hand within the last 6 months, uncorrected vision or hearing problems, and memory impairment as defined by having more than two errors on the Pfeiffer short portable 
mental status questionnaire (SPMSQ) (Pfeiffer, 1975). The SPMSQ has 86.2\% sensitivity and 99.0\% specificity (Erkinjuntti, Sulkava, Wikström, \& Autio, 1987) in community dwelling residents and people with dementia for the cut-off scores used to screen participants in this study without the correction for education.

The goal was to have an equal representation of each of the four diagnostic groups and gender among participants. A varied range of hand function difficulties among 16 participants in focus groups and eight participants in cognitive interviews, spread out equally among four diagnostic conditions was planned. The sample size of focus groups was intentionally kept larger to allow thematic saturation (Marshall, 1996). The sample size of eight for cognitive interviews was determined to be sufficient as the focus groups and cognitive interviews in this study were to be used to qualitatively assess individual items prior to their inclusion in the HAFM, not to perform any statistical procedures.

Procedures. Participants came to the University of Washington Department of Rehabilitation Medicine to participate in the focus groups and cognitive interviews. At the beginning of the cognitive interviews, demographic data was obtained and the following standardized self-report measures were completed either at the beginning or end of the session: MAM: Manual Ability Measure-20 (MAM) (Rallon \& Chen, 2008), Patient Reported Outcome Measure Information System ${ }^{\circledR}$ (PROMIS) Fatigue short form 7a version 1.0, PROMIS Depression short form $8 \mathrm{~b}$ version 1.0, and PROMIS Pain Interference short form 6b version 1.0 (Broderick et al., 2013). Focus group and cognitive interview data were collected from July 2014 to August 2015. The focus groups and cognitive interviews occurred approximately one year apart. The focus groups were aimed at exploring what aspects of hand impairments affect activity and participation and what the participants' experiences have been with formal or 
informal testing of hand function. For example, one question in the focus group was, 'What are some of the daily activities, very important to you, that you cannot do with your arm and hand?' A semi-structured interview guide was developed for focus groups and is presented in Table 4.1. In the cognitive interviews, participants with neurological condition completed 10-15 performance tasks and self-report items, and 'talked aloud' about their perceptions about the tasks and items in an interview. The interviews at this stage were focused on item and task clarity, response choices, and context perceived by the participants with neurological condition in response to the question. A semi-structured interview guide was developed for cognitive interviews and presented in Table 4.2. For example, one question in the cognitive interview was 'How could the task instructions be improved?'

Materials. For the cognitive interviews, the materials (shown in Figure 4.1) needed for administering the performance-based section of the HAFM cost $\$ 46.56$ and included: Measuring tape, paper tape, teaspoon, mug, can, pencil, pennies, lock, key, peas, jar, bottle, bowl, socks, beans, paper clip, nut, bolt, and paper pad. The details regarding the quantity, brand, size, and type of item is provided in Table 4.3.

Analysis. Data immersion, coding, category creation and thematic analysis were used to find patterns of meaning across data (Thomas, 2006; Schmidt, 2004). An inductive approach was used to derive themes by interpreting the raw data. Codes and sub-codes were identified by two researchers (NG and DK) independently. The researcher's notes during and after the session were not coded. The cognitive interview codes were tied to the items that were administered and further coded based on keeping the item or not and changes needed to implement. To organize and manage the data, the qualitative analysis software program Dedoose version 6.2 (www.dedoose.com) was used. 


\section{Results}

A descriptive qualitative approach was utilized for seven focus groups and eight cognitive interviews with purposeful sampling of participants. Seven focus groups involving two to four participants and one to two researchers were conducted. Eight cognitive interviews involving one participant and one to two researchers were also conducted. Table 4.4 presents the characteristics and demographic data of the participants. Of the total of 20 participants, six participants (participant 19, 20, 22, 23, 24, and 25) participated in both a focus group and a cognitive interview. There was fair gender representation with $45 \%$ female (9 out of 20 ) participants. The average age of the participants was 53.81 years with age ranging from 29 to 72 years. The educational background of $95 \%$ of the participants was high school or higher. The mean level hand function of the participants on the converted score of the Manual Ability Measure (MAM) was 61.35 out of 100 (ranging from 47.8 to 100). Figure 4.2 presents the graph showing distribution of MAM scores by diagnosis. The PROMIS fatigue, pain and depression scores of participants are also reported in Table 4.4 to better understand the neurological condition of the participant and their functional level for a useful interpretation of the views expressed in the study.

All audio interviews were transcribed verbatim and thematically analyzed. For coding and thematic analysis, there was overall good agreement (focus group: $96.09 \%$ and cognitive interview: $89.35 \%$ ) and the points of disagreement (focus group: 3.91\% and cognitive interview: 9.13\%) were resolved by discussion by the two researchers. Disagreements identified by the excerpts that were coded as 'query' or 'discuss' in the transcripts and subsequently resolved through discussion. There was consensus between both researchers on the final coding scheme as being representative of the data. 
Focus group themes. The participants in the focus groups discussed the importance of remediation of hand function. The participants shared prior experiences with therapy and life experiences in general related to their hand and arm function. There was agreement among participants that there is a general lack of awareness for problems related to hand and arm function as discussed in the following piece of discussion among participants.

I think this is an overlooked area and that's why (in response to thanking for being a part of this study). I was explaining to my wife, the only thing you can't do without your legs is go for a walk in the park. But if you don't have hands you can't even wipe your own butt. (Participant 11, MS)

That's very true. (Participant 13, MS)

Why is the wheelchair the biggest fear? There is something psychological about it. (Participant 11, MS)

Eight key themes emerged from analysis of the focus group transcripts.

Conditions. A participant with TBI talked about his bilateral involvement, shakiness of hands, co-morbid seizures, anger issues, and his frustration with hand problems at 29 years of age.

I like to watch sports stuff like that. I want to get into fishing again. But I only have one arm so everyone is like, no you have two arms. But I am like no I just have one arm. Because I pretty much consider this one dead. Mainly in my head I wish this TBI, this hand would have gotten cut off or something. (Participant 18, TBI) I cant use my this (left) hand. I can still use my right hand to do everything. Even though I can't really use it (right hand) just because it shakes so much you know. But it's the only one I have so I use it. (Participant 18, TBI) 
Participants with PD talked about the differences among people with PD and that no two individuals with PD are alike.

The things (about differences between people with PD previously said) that Participant 7 said so eloquently, it's different for everybody. And your progression is different for everybody. (Participant 5, PD)

That's why they call it a snowflake disease. (Participant 7, PD)

Ya. (Participant 5, PD)

Snowflake disease? (Researcher, NG)

No two snowflakes are the same, no two Parkies (referring to people with PD) are the same. I don't know if it matters but its true. (Participant 7, PD)

Some of the symptoms discussed by participants with PD were ataxia, cramping, dyskinesia, micrographia, rigidity, feeling numb, freezing, slow movements, sudden jerky trigger movements, tremors, voice changes, fixed postures of the hand, and issues related to progression of the disease.

I keep trying to do this, (showing a hook fist) to not show my tremors. (Participant 6, PD) Oh the 'Parki-claw.' (Participant 7, PD)

Participants with stroke talked about the tone, co-morbid seizures, the road to recovery of function, the change of dominance, and coping strategies used by them. Participants seemed to appreciate sharing the stories of their recovery in the group. Participants with MS talked about the episodes, relapses, heat intolerance, optic nerve problems, variable presentation and issues with progression. These condition specific symptoms prompted the introduction of a symptom inventory at the beginning of the self-reported section of the HAFM. Further, to acknowledge the 
different symptoms on the two sides of the body, the questions would separately ask for left and right hand symptoms for all conditions.

Compensation and adaptation. There was a lot of discussion about the compensatory strategies used by participants, including demonstrations of adapted tasks such as picking up golf tees, playing with a ball, and holding two grocery bags. As a result, compensation was included as one of the aspects within the response options and therapist was provided space to record notes during session related to compensation or abnormal movements.

Dominance. Some participants reported the strong sense of loss due to loss of their dominant hand function.

It is so strange. I want my left hand back so bad...(Participant 8, stroke)

Some others reported that their non-dominant hand function was not well trained since the dominant hand overpowered them. It was clear that function was very different for people with dominant hand more affected than the non-dominant hand.

Items. There were 160 item codes that generated 145 items in the item bank presented in Appendix D. Some codes were grouped together to form items, for example, handling bags, purses and backpack were grouped together as a subcategory within 'object handling.' There were nine main subthemes within which the items were grouped: i.e., carrying, communication, instrumental activities of daily living (IADL), movements, object handling, reach, recreation, self care, and transportation. Carrying involved being able to carry items like groceries, coffee cup, wine glass and bag from one place to another and involved endurance of upper extremity to hold a sustained contraction and keeping the hand steady during movements involving rest of the body. Communication represented using hands as tools of communication for clapping, shaking hands with another person, and writing. 
My handwriting has definitely gone downhill. I can't read it any more. I have a diary, but I can't read what I have written on the diary. (Participant 14, PD)

But I would say clapping looks a little sloppy and it's been that way for a few years. I noticed it because when teaching children and clapping along, I kind of have to find a flat part to clap. (Participant 17, MS)

My wife laughs at me when I am clapping, she is like - "Is that it" (making a low and flat sounding clapping sound). At the Sounders game, I am like cheering, that's pathetic (claps). (Participant 16, MS)

IADL tasks include activities related to independent living such as childcare tasks, grocery, housekeeping, kitchen tasks, laundry, work and others. The group of individuals in this study were community dwelling individuals who wanted to regain or maintain their ability to engage in IADL tasks. Movement tasks involved the movements that were articulated by the participants such as holding, lifting, opening and closing hand, picking up, placing on table, and tapping on table. Object handling included all the different objects that were mentioned by participants as challenging to manipulate due to hand and arm issues. Some examples of objects were a ball, bottle, cans, child safe medication bottle, coins, credit cards, cup, doors, jars, and keys. A sub-theme within object handling was handling of technology related objects such as computer, tablet, phone, keypad, mouse, typing and touch pad. Reach included reaching to a shelf and taking a box off a shelf. Recreation included various leisure time tasks that were relevant for participants, and were very varied. If the measure was to address recreation, participants expressed there had to be a way to capture the specific leisure task that was relevant to the individual. This was also evident for the theme of work as well. Participants expressed 
offering a way to capture the work related tasks that are most important to the participant emerged as an important component of the measure.

Hand function. The participants had varied perspectives on the various functional aspects of the hand.

Bilateral tasks. Participants were trying to use the affected hand in functional tasks and reported that they resort to doing one-handed tasks only when faced with time or activity constraints.

Ya, I was always right handed. And I said the main thing that was difficult was cutting. Doing laundry, I have a hard time pulling it out of the dryer. I could use both hands but this hand is very weak so if I need to get things done fast I just use my dominant hand.

Which is not helping my non-dominant hand. (Participant 10 with stroke)

You can't clap. (Demonstrates clapping) (Participant 2, Stroke).

So does that bilateral function matter? Two handed. (Researcher, NG)

Oh yes, yes. It's a hassle to do one handed. (Participant 2, Stroke)

Doing activities with both hands was important to the participants and to some extent it was their expectation from a therapy session that the therapist would want to learn more about their affected hand and aim to tailor the session to improve function.

Low level of hand function. The participants expressed their perspectives about very low hand function in terms of relief of symptoms and passive movements.

I have, in trying to make my left arm do things, it activates spasticity, which I get Botox...I actually don't have any hand function except, I can push something. ...The Botox relaxes the muscles? It makes it so that even if I cannot do it willfully, I can do that 
(shows passive opening the hand and straightening the elbow, flexion of shoulder). It doesn't hurt. (Participant 2, Stroke)

The passive movements were not included to avoid ceiling effects in the items in the measure.

Strength. Hand strength was one of the most commonly identified aspects of hand function and included grip and pinch strength in the discussion. The most commonly tested aspect of hand function in the clinic that all the participants could identify was grip testing. Some hand function measures such as the Wolf Motor Function Test include grip testing with dynamometer along with the other items in the measure. However, it was not considered for inclusion in this new measure of hand and arm function because of its instrumented nature. Daily activity tasks like picking up bean can and using a key were considered representative of grip and pinch strength respectively for this measure.

Sensation. The participants linked aspects of hand sensation to the hand function discussion. Aspects such as estimating the force for gripping, proprioception for knowing the hand position, tingling, and having to use vision to compensate were discussed.

I have to really look at it (the paper cup). Keep my eye on it. But if I turned away, I wouldn't realize that I have just crushed it and spilled it. But if I really focus I could do it (keep it) right there and put it there. But I wouldn't get up and walk with it or anything. (Participant 16, MS)

The final items thus included sensation aspects to get a patient and therapist reported measure of sensation. 
Day to day changes. The participants brought forth this unique perspective of day-to-day changes in their hand function and even general health. This view was shared by participants with MS, PD and stroke, as seen the following quotes.

So there isn't a single activity yet that is completely gone. At times I have more or less difficulty. Or more or less sensation and I am just constantly, you know stretching and very conscious of my hands. (Participant 11, MS)

So I mean good days and bad days, we all have them regardless of what kind. (Participant 15, PD)

So its hard to take that variable out of the equation. (Participant 14, PD) Well some days I wake up and you just cant sit still and some days I can be fairly calm most of the day and it affects your energy level because this takes energy when you are moving all the time. (Participant 14, PD)

Ya sometimes it feels like it gets stronger but I feel I do have sensation in it but I can feel right now. Some other times I am not aware where it is. Like my boyfriend was saying he will say when we are sleeping I hit him in the face. (Participant 10, Stroke)

The self-report questionnaire seemed sufficient to capture the variations in function. Thus, this feedback was not incorporated for the measure during cognitive interviews. As our understanding of the way to incorporate this feedback developed after the cognitive interviews and was supported by participant 26 with TBI's suggestion of asking in the questionnaire if this was a typical day, this question was incorporated in the revision after cognitive interviews.

Functional categories. Participants also identified aspects of hand function such as strength, dexterity, accuracy, coordination, endurance, fine motor, hand movements, range of motion, reaching, speed, tone, tremor, turning the hand, pain, and neglect. 
So you have strength and dexterity, and accuracy. (Participant 5, PD)

What's accuracy? (Researcher, NG)

Being able to punch the button as opposed to the one next to it. And then repetition.

(Participant 5, PD)

Participants helped describe these aspects. Patient's familiarity with some of the commonly used therapy jargon also supported the idea of including some therapy jargon within the measure. For example, people with PD were familiar with tremors and incoordination and people with stroke were familiar with tone and spasticity.

In the discussion of sitting versus standing for doing tasks, some participants' thought standing and doing tasks was harder due to poor balance.

Oh ya you've got your balance to worry about to stand. (Participant 4, PD)

Standing ya and now you are dual tasking. (Participant 7, PD)

Another participant thought sitting and doing tasks was harder since her shoulder would work best in standing to do the task and be able to generate more force.

Depends on a task I guess. If its sitting at the kitchen counter, you can stand and do it, that might be similar but if its coming from your arm the function is going to be different here than up here... Say like when you are cutting, your shoulders would be working all the time sitting. (Participant 1, Stroke)

I was thinking its easier standing. (Participant 2, Stroke)

For the measure, all tasks were administered in sitting to avoid the influence of standing balance on the items. Sitting balance was not discussed in this group as none of the participants had trunk control issues. Sitting balance might need more investigation through qualitative data in the future for this measure. 
Hand use versus function. One of the questions from semi-structured interview guide was related to a discussion of terms "hand use" versus "hand function."

I don't know what the difference is. (Participant 2, Stroke)

Function to me says it can function. (Participant 1, Stroke)

Function is specific, like you have fine motor dexterity, more than usage how much

should I be able to use it. What does use mean? Because people compensate and are able

to get the task done but the motion so that they are not using the certain muscle.

(Participant 17, MS)

I like the word function, there is a much greater meaning than use. Use is like I think is so general. (Participant 16, MS)

There was a repeated affirmation from participants that they understood what a hand function measure would entail. Hand use seemed more ambiguous to the participants.

Medication. Medication management seemed to be a very important piece of discussion for the people with MS and PD. Moreover, for PD there were direct effects of medication wearing off referred to as the 'off' phase versus the 'on' phase of good function under the influence of medications. It was clear from repeated reporting of medications and their effect on function that it was important to find out if the participant was in their 'on' or 'off' phase. For this, it was important to know more about the medication taken before the administration of a measure. Thus, space to report medications was added to the patient information section.

Test. We were interested to find out if participants had any opinions about their hand function being tested. Some voiced their concern about testing. 
I am not sure I capture how uncomfortable it can get to be measured... We don't need the measurement. It is for the clinician, not for us. It is not even for the clinician, it's for the clinician's supervisor to tabulate results. (Participant 11, MS)

Don't test, don't just test without any offering. (Participant 11, MS)

The participants indicated they wanted the outcomes of tests being used in some form when probed about results of the tests that participants have undergone.

Well I don't think I have seen any results of any of the tests (of hand function) I have done. I mean there is not a pass/fail. That you just plug the information in and somebody grades it in some aspect but I don't think it relates to me as an individual. (Participant $15, P D)$

Would you like to see those? (Researcher, NG)

Doesn't make a difference. (Participant 15, PD)

Some participants did not want to know the results possibly because of a progressive disease course. Thus, it was decided that the participant should be offered the choice to know about their test results. Some participants also requested a comparison of the current performance to past performance and a comparison of performance between the two upper limbs.

The normal range of scores and where I am at now. Ya do it separate, one arm and then the other arm. (Participant 18, TBI)

Participants offered many suggestions about the measure. They suggested, it would be helpful if the self-report section be administered before the performance-based section; and that pictures be used to augment verbal instructions. The participants also spoke to the importance of having items that are relevant to their lives and that a variety of tasks be included. They further 
expressed the importance of being able to track the performance of their left and right hand separately and be able to track change over time.

When asked about being timed, some of the participants mentioned that they did not like being timed at all since it adversely affected their performance as discussed in the following quote.

Well when I am timed, I find myself not able to do it as well. (Participant 14, PD)

The stress of the time, you have to get done in a certain amount of time and then all of a sudden I cant do it. If I have the time to do it. (Participant 14, PD)

Other participants enjoyed the competitive aspect of being timed and would try to beat it. In view of the importance of time to track small changes in performance, particularly tasks needing accuracy, it was decided that only dexterity related tasks would be timed.

The complete item bank is presented in Appendix D. These items were then organized by the frequency with which they were coded in the transcripts. Since there was only one individual with TBI, the frequencies for the item codes were adjusted to a value of one to avoid overrepresentation of any one single code (e.g., the code 'Carrying a coffee cup' occurred three times for the individual with TBI, however it was adjusted to a value of one). The items from expert panel study and focus groups were pooled. In order to pool the items, the item codes were kept consistent across the two studies. The top $1 / 3^{\text {rd }}$ of high frequency of the pooled items were selected as the final item set. The 59 identified items were then sorted into self-report and performance-based categories based on testing performance for items that are critical for movement quality, dexterity and speed. The item development process is presented in Figure 4.3.

The final set of items for the cognitive interviews had 11 symptom related questions, 34 ADL questions, and 14 performance-based tasks. The reading ease according to Flesh-Kincaid in 
MS Word was $74.4 \%$ at a 5.5 grade level for version A of the self-reported section of the measure and $73.4 \%$ at a 5.7 grade level for version B of the self-reported section of the measure. The reading ease for the performance-based section was not calculated since the target audience reading the document were therapists who were familiar with higher reading levels and medical terms needed for explanations. These measures are presented in Appendix E for self-report section of the measure and Appendix F for performance-based section of the measure.

Cognitive interview themes. The participants made varied suggestions to changes to the items, wording and tasks. Most questions were presented after participants completed four to five items. The feedback from the interviews resulted in substantial changes to 39 out of 59 items with three major themes; new tasks, changes suggested for items, and changes suggested but not implemented for the items.

New tasks. Participants suggested new tasks as they remembered other tasks related to the items. These are presented in Table 4.5.

Now you don't have tying a tie on there. ... that's a big one. It's a big endeavor, (to tie a tie) it takes half an hour. (Participant 23, Stroke)

This quote highlights the suggestions for specific tasks that was important for this male participant and could not be incorporated in the measure since female participants would not be able to attempt to task. It was discussed by participants that medication sorting task into pill box for the week was a meaningful task and this being a universal task was added in the list. ICF domains of throwing catching, pushing, pulling, were not included in the measure and will be incorporated in the self-report section. Release of the fingers to release an object was not included as a specific item but the response options do take into account the ability to release the object. 
Changes suggested for items. The final set of 59 items administered during the cognitive interviews had 11 symptom related items, 34 ADL items, and 14 performance-based task items. Each item was administered to five to seven participants, ensuring all items were tried by at least one individual in each of the four diagnostic groups. The items were split into version A (Selfreport: 34 items, Performance-based: 10 items) and version B (Self-report: 29 items, Performance-based: 10 items) with overlapping items. The version A for both sections was administered before version B for five participants, and in the opposite order for three participants. 14 performance-based tasks were split into 21 items since some items were administered on both sides, right and left (e.g. touching the back of the head on right and left). The changes suggested by participants were also coded for the item for which the change was suggested. Participants were probed about the burden of the items and of being in a testing situation as a whole. None of the participants felt that the items were overwhelming or that too many items were presented to them.

There were six sub-themes within the implemented changes; i.e., items, response options, scoring, demographics, symptoms, and medications. The sub-themes represent the items in the self-reported section and are followed by the performance-based section to organize the information.

Items. In the self-reported section, optional items were liked by the participants but they suggested the format needed revision. Moreover, the childcare and school item did not seem to of particular interest, even among people who were engaged in daily tasks related to them. Thus, it was decided to remove the two items. The work and recreational items were preserved in a changed format that allowed the participant to list an item and rate it without rating the item in general. The item related to tying shoes was preserved and putting on shoes was removed due to 
confusing wording and redundancy. The item related to 'wallet or purse' was changed to 'wallet or purse or pocket.'

In the performance-based section, the 'hand to lower back' item instruction wording was revised for reaching to the back at the waist to avoid confusion as to where on the back to reach. Pick up the coffee mug needed more clarification of 'using the handle' to avoid a person from not using the handle since handle needs more coordination. In the item 'pennies on table,' pennies to be stacked on the table or not was not clear. For the sensory item, the pictures of the objects were provided for patients with difficulty naming objects. One participant observed that the names of those items should come right under the pictures. Moreover, if pictures were shown to participant when testing one hand, for example right, the same object should not be used for the left hand otherwise the participant gets a hint as to what items might be in the sock during testing of the left hand.

The order of the sensory items needed attention. The cotton ball was a difficult item to identify for the participants and was only presented on one side occasionally. It was perceived as unfair if the affected side encountered the cotton ball. Thus, the varied texture and temperature of objects was perceived as inconsistent in the test. A clear solution does not exist to this issue; however keeping the same texture for all objects is possible. For example, if all objects were metal like key, coin, nut, or bolt then the test would be consistent for the material and remembering the type of object could be avoided with repeated testing. Some of the items in the test were bimanual items (e.g., opening a lock with a key), and participants were given a choice to use the key in any hand. Not surprisingly, participants held the key in the less affected or unaffected hand. The task was originally designed this way since the participant has a choice for which hand to use in real life. Thus, the measure would reflect their real performance. However, 
participants voiced their concern with this idea. They argued that their purpose in therapy is to gain more functionality of the affected hand. This can only be achieved if the therapist identifies the problem when testing them with the HAFM. Thus, the suggestion by participants to test their ability to do the task both ways, i.e. key held in affected and unaffected hand alternately, was accepted. The bean can task needed major revisions including improving the clarity of the picture provided for set up, extending the reach with the bean can beyond the two 6 inch markers, and planning pick up and place of the can to coincide with start and end of the task. Moreover, the bean can task did not intend to represent carrying and strength of grasp domains of hand function as originally planned. It did represent the carrying domain, but the grasp was redundant with the grasp for the jar, and a separate strength task such as 'pouring water from a pitcher' or 'picking up a half gallon of milk' would be added. The opening jar and opening spice bottle task involved similar movements and were redundant. Thus, the more commonly performed opening jar task was preserved and opening spice bottle task was removed.

Response options. In the self-report section, the participants suggested adding a comment section in order to indicate specific details about the tasks as they mark the options, and remember the detail at that moment. The three options for response given in the self-report section of the measure were not at all, a little and quite a lot. More than one participant indicated that their function was more impaired than quite a lot. Another category of response option 'unable to do' was added to address this issue. If response options are not clear, therapist can help the participant problem solve the degree of difficulty and this instruction needs to be added to the manual.

Scoring. In the self-report section, the numbers that were meant for therapist to calculate the score were very confusing for participants and some tried to count the score themselves. The 
self-report measure did not specify if the participant had to mark the response with a check or a cross. The commonly used check mark option was added.

For the performance-based section, there was no way of recording follow up assessments unless a new form was used. The assessment form needs to have the ability to record an initial, follow up and discharge assessment. Participants reported that a numerical score with nothing to compare against was not helpful. A qualitative summary that they did well on a skill such as reaching and average on a skill such as fine motor would help them start a conversation with their therapist about their goals. This was possible to implement for the performance-based section since the task design could be tailored to the function to be assessed. The combination of hand function aspects on the performance-based section and daily activity domains on the selfreport section might give the clinician a better picture to further investigate in the therapy session. Moreover, it was decided that the manual would include suggestions for the therapist to offer the client/patient a chance to choose one item that is most relevant to them to follow up over time in a numerical form.

Set up. The set up needs were only for the performance-based section of the HAFM. One of the participants had a skin condition and this brought to attention the need to add explicit wording about cleaning the test items in the manual. The administration of a new measure of hand and arm function required the reading out of standard instructions to the participant, however it was necessary to tell the participant that the therapist will be reading out the standard set of instructions in order to clarify the administration process to participant.

Demographics. The demographics information was entered by the participants for the self-report section of the HAFM. A participant remarked that the 'date' be changed to 'today's date.' The word 'hand preference' was preferred over the word 'dominance' by participants. The 
word diagnosis was difficult to understand for some participants and will be defined in the measure.

Symptoms. In the self-report section of HAFM, meanings of the words spasticity, hypersensitivity, and deformity were not clear. However, there were some participants who were more familiar with these terms possibly because they experienced the symptom and frequently used it with clinicians. Thus, there was a need to accurately pose the question related to the specific symptom but there also was a need to define the term for people who were unfamiliar with it. It was thus decided to add descriptions of the terms spasticity, hypersensitivity and deformity in the revision of the measure. Participants remarked that on the self-reported section, the questions start out with symptoms on right and left hand and then move on to items that are assumed to be bimanual. This order was confusing for the participants. One participant suggested that the right and left hand separation should happen in the end to avoid confusion. Thus, the order of the tasks was changed to move symptoms towards the end of the measure. More than one participant suggested that a reminder that all questions are targeted towards the past week is needed at the beginning of each page. For the Ziploc ${ }^{\circledR}$ item, opening a Ziploc ${ }^{\circledR}$ was reported to be harder than closing thus closing the Ziploc ${ }^{\circledR}$ was removed from the item. It was suggested that we use the word typing as opposed to keyboarding to keep the term more general.

Medication. For self-report section, it was clear from patient reporting that not only providing space to enter the medications taken by the patient was meaningful, but also the timing and dosage of medication influenced arm and hand function and was important to note. It was decided to expand the space needed for entering medications on the patient information section before the administration of the measure. Also, patients were occasionally confused about the 
medication or dosage and an instruction in the manual was to be added to request patient to bring a list of medications to the therapist before session.

Changes suggested but not implemented for the items. In the self-report section, the participants suggested that symptoms should only be listed as right and left, however in its current form, the terms 'right hand' and 'left hand' remind the participant that the symptoms probed are related to hand function.

In the performance-based section, it was suggested by one participant that writing a check was a good activity for high functioning individuals with PD to bring out the micrographia. However, since the writing task is timed, it was assumed that the time factor would capture the differences between high and moderate function. One participant suggested stacking the coins to pile them up as a modification to the penny on table task. Given the potential increased length of time it would take an individual with moderate to low hand function to complete making stacks of coins, this suggestion was not implemented. One participant suggested that the items that were difficult for her should be completed with assistance to help her learn the skill. This suggestion was also not incorporated as it is contrary to the purpose of the measure to identify the limitations in function and it is not desirable to teach the tasks of the measure to the participant. Activity limitations are more appropriately be the target of therapy. For the same reason, another suggestion that was not implemented was the use of a non-skid pad or mat.

Finally, the 13 self-report items that worked well were in the subsections of self-care, technology, kitchen, and miscellaneous tasks. The current form of the performance-based items worked well for seven tasks, i.e., touching back of the head, getting the coffee mug, getting the jar, opening the jar, picking up spoon, and holding the pennies. 


\section{Discussion}

This qualitative study aimed to explore the opinions of adults with neurological conditions regarding hand and arm function in daily activities and their perceptions about a preliminary set of items to measure hand and arm function. The focus group discussion helped identify aspects of hand function that were relevant to the participants with neurological condition such as sensation, strength, grasping, and fine hand use. The feedback from participants helped develop the item bank. The item bank from participants with neurological condition was then pooled with the items from expert panel to identify the high frequency items were included in the preliminary set administered to the participants during the cognitive interviews. Based on the cognitive interviews it was found that self-report and performancebased aspects of function were well received by the participants. The performance-based items needed to be tailored towards impairments on the affected side along with preserving the bimanual nature of the tasks. The self-report items needed definitions of the terms included in the questions, an additional response option and a comment box, and a change in the order of presentation of items with bimanual followed by unimanual items. Focus group and cognitive interview probing also helped shape the construct with more attention to the affected side and to the aspects of the lower end of hand function. The problems with the items resulted in modification of items or removal of the items that were redundant (e.g., opening jar and opening spice bottle) or did not work as intended by the researchers (e.g., interference of hand function with childcare).

The participants expressed liking the 'not applicable' option in the self-reported measure. There have been prior studies (Morgan, Amtmann, Abrahamson, Kajlich, \& Hafner, 2014) where participants have reported the usefulness of having a 'not applicable' option. A not applicable 
option however, can present potential scoring issues that may reduce the quality of data (Krosnick et al., 2002). However, in the absence of a 'not applicable' option, participants may provide inaccurate information or leave a question blank. In both of these situations, the quality of the data may be threatened. Providing a 'not applicable' response for an item of hand and arm function that a participant may never encounter in their daily life (e.g., an item like 'washing hair' when a participant did not have hair) is consistent with the assessment framework of this measure.

This study contributes to the literature and demonstrates the involvement of stakeholders in the development of a measure of hand and arm function. This is consistent with the guidance for researchers by PCORI (Patient-Centered Outcomes Research Institute, 2012)and large regulatory bodies like the Federal Drug Administration (FDA) (U.S. Department of Health and Human Services FDA Center for Drug Evaluation and Research et al., 2006). It also aligns with the 8-stage framework presented by Velozo and colleagues (2012) who also advocated the use of mixed methods in preliminary research (Velozo et al., 2012). The use of focus groups and cognitive interviews with people with neurological conditions is the strength of the process of instrument development for the HAFM.

There were some differences among people with different neurological conditions. The people with stroke, stressed recovery and the effect of spasticity issues. The people with TBI talked about ataxia and participation in higher level work related issues. The people with PD were very concerned about the effects of medications on function, particularly tremor and incoordination. The people with MS were concerned with issues related to progression of the disease and the lack of awareness among clinicians for hand function problems. These 
differences have been acknowledged by some authors (Lundy-Ekman, 2012), but not used for measure development in hand function.

Some studies have used formal reading frameworks for the calculating reading level (Devraj \& Wallace, 2013). The ease of reading the instructions and questions was calculated for the self-report measure, but a reading level using a standardized reading framework was not used in this study. The need to further evaluate was not sensed given the documents were at the reading grade level according to Microsoft Word's Flesch Kinkaid Reading Level of 5.5 to 5.7 for the two versions of self-report. The diverse literacy levels of cognitive interview participants based on self-reported educational level from less than high school to doctoral degree was a beneficial aspect in this study to identify the ease of language and minor nuances in wording and format.

Limitations. Not uncommon to qualitative studies, this study is limited by influences of personal researcher biases (Anderson, 2010). A detailed log of researcher's notes post session were kept with the intention of recording any biases and involvement of the second researcher during analysis were attempts made to neutralize this effect. Moreover, the primary investigator was an occupational therapist and second researcher was a physical therapist, which helped reduce some of the inherent biases related to perceptions of daily activity limitations seen in occupational therapy practice. Also, a group of 20 participants may not be representative of all the views within the community of people with neurological conditions.

There are other ways to established content-related evidence for validity of scores (Lawshe, 1975), like quantitative analyses. Lawshe (1975) has suggested creating a survey for the expert to comment on each item with one of the three responses - essential, useful but not essential, and not necessary. The responses of the experts are pooled and the number indicating 
'essential' marked for each item is computed. Although, this approach provides quantitative data for item selection, the sample size of experts in this study was not sufficient to design a quantitative study. Moreover, no added benefit was perceived in structuring in a quantitative format over qualitative format for data gathering when the open ended questionnaire had more room for experts to present their opinions, suggest new changes and suggest new items if needed.

Some theorists (Netemeyer, Bearden, \& Sharma, 2003) have argued that theoretical framework provides the best conceptualization and discounted the benefit of participant feedback. However, participant feedback supports the evidence for validity of scores and use of the measure. The field of clinical testing is very much client-centered and must consider the input of the patient or client in scale development.

The self-reported and performance-based formats of tests themselves present with strengths and weaknesses. The major drawback of self-report format is the lack of faith of clinicians in accurate reporting by the client or proxy. However, NIH initiative on PROMIS has clearly emphasized the need for more patient-reported outcomes. The main reason for this initiative is the involvement of the client in the rehabilitation processes of assessment, setting goals, treatment and discharge. The self-report itself can start a dialogue regarding the problems encountered by patient or client that can be addressed in therapy. The performance-based format is traditionally well accepted by therapists and gives the therapist an objective measure of patient or client's hand function in the areas tested. However, it is a measure of capacity and does address the varied daily tasks encountered by the patient or client in routine activities.

The hand function level of participants was 47.8 to 100 converted score on the MAM and it is speculated that individuals with low functioning were not adequately represented in this group. Thus, it is possible that a complete assessment of function was not done. Ongoing 
research will need to focus on recruiting participants with lower hand function to address this issue. Also, individuals with TBI were not well represented in this group with a total of two participants. Further efforts need to be made in order to recruit more participants with TBI in future studies. Also, the regional nature of sampling may place limits on the generalizability of the findings.

Although the participants did not report that the measure was burdensome, it may not be an accurate representation of how the measure would be perceived in the clinic. The participants in this study were volunteers who seemed to care about the hand and arm function issues in neurological conditions, which was the reason they were participating in this study. Further, the participants had two hours to complete the items and discuss them, which is very different from pressures of a busy clinic. Thus, clinical testing needs to be done in the future to identify the optimal number of questions that will not feel burdensome to the participant and therapist in a clinical environment.

\section{Conclusion}

The focus groups and cognitive interviews facilitated the grounding of the HAFM in the views of participants with neurological conditions thus helping to establish the face and contentrelated evidence for validity of the scores of the new measure. Psychometric studies are needed in the future for measuring evidence for the reliability and validity of scores of this measure in assessing upper extremity function in people with neurological conditions for its intended use of tracking progress over time. 
Table 4.1. Semi-Structured Focus Group Guide for Participants with Neurological Condition

General measurement questions

What came to your mind when I said arm and hand use?

What if I would have said arm and hand function? What would that mean to you?

What is important to measure about arm and hand use? Why? How?

Do you remember any tests of arm and hand function that you have taken in the past?

Can you tell me about them?

What if a test had a questionnaire to fill out and a portion where there were timed tasks that you would have to do, would it make sense to have both in a test?

In what form would you like to know the results from a test of arm and hand use?

Specific questions related to the neurological condition

How does your neurological condition affect upper arm use?

How does your neurological condition affect lower arm use?

What are some of the daily activities very important to you that you cannot do with your arm and hand?

Do bilateral activities matter to you?

What about dominance? Were you right or left-handed? Did that change with the diagnosis of your neurological condition?

Additional questions and discussion may follow based on the level of detail provided by each group member and flow of the conversations. 
Table 4.2. Semi-Structured Cognitive Interview Guide for Participants with Neurological Condition

Performance-based measure questions

What were your first impressions about the tasks?

Tell me how difficult you thought the tasks were?

How could the task instructions be improved?

Tell me how relevant the tasks are to you?

What do think about having some of the tasks timed?

What if any tasks should be included in this test?

Self-report measure questions

What were your first impressions about the questionnaire?

How was the wording of the questions?

Tell me how relevant the questions are to you?

What about the time frame of "in the past week"?

Tell me how the response options worked for you?

What if anything specific to your condition should have been on the questionnaire?

Additional questions and discussion may follow based on the level of detail provided by each group member and flow of the conversations. 
Table 4.3. List of Materials for Performance-Based Section of Hand and Arm Function Measure

\begin{tabular}{|c|c|}
\hline Material & Type \\
\hline Adult tube pair & Black adult tube sock pair Hanes ${ }^{\circledR}$ Brand: Length: 12 inches \\
\hline Bean can (unopened) & $\begin{array}{l}\text { Bush's Best }{ }^{\circledR} \text { brand dark red kidney beans can, paper removed } \\
\text { during test: Capacity }=16 \mathrm{oz} \text {., Weight }=1 \mathrm{lbs} \text {. }\end{array}$ \\
\hline Blackeyed peas (15) & Goya ${ }^{\circledR}$ brand dry black eye peas \\
\hline Bowl & $\begin{array}{l}\text { White porcelain cereal bowl Correlle Livingware } ₫ \text { brand: Capacity } \\
=18 \mathrm{Oz} \text {, Inner rim Diameter }=6 \text { inches }\end{array}$ \\
\hline Coffee mug & $\begin{array}{l}\text { Porcelain coffee mug with one handle on the side, Correlle } \\
\text { Livingware } \AA \text { brand: Capacity }=12 \text { oz., Height } 4 \text { inches }\end{array}$ \\
\hline Coins - pennies (16) & US currency penny $=1$ cent \\
\hline Dry kidney bean & Goya ${ }^{\circ}$ brand dry red kidney beans \\
\hline $\begin{array}{l}\text { Jar with a screw top lid } \\
\text { (contents sealed) }\end{array}$ & $\begin{array}{l}\text { Skippy's }{ }^{\circledR} \text { brand peanut butter jar: Weight }=1 \text { lbs., Capacity }=15 \\
\text { oz. }\end{array}$ \\
\hline Key & $\begin{array}{l}\text { Metal key part of the Masterlock } ® \text { brand no. } 2 \text { set: Length }=1.6 \\
\text { inches }\end{array}$ \\
\hline Key and a padlock & $\begin{array}{l}\text { Masterlock } ® \text { brand No. } 2 \text { padlock: Width }=11 / 8 \text { inches, } 29 \mathrm{~mm} \text {, } \\
\text { Shackle height }=1 / 2 \text { inch, } 13 \mathrm{~mm} \text {. Key: Length }=1.6 \text { inches }\end{array}$ \\
\hline Measure tape & Stanley ${ }^{\circledR}$ brand key chain tape measure \\
\hline Nut and bolt & Nut: Diameter $=1 / 2$ inch. Bolt: Diameter $=3 / 8$ inch. \\
\hline Paper clip & Paper clip: Length $=1.1$ inch or $28 \mathrm{~mm}$ \\
\hline Paper tape & Curad $\AA$ brand gentle paper tape: Width $=1$ inch \\
\hline $\begin{array}{l}\text { Pencil (at least } 6 \text { inches } \\
\text { long) }\end{array}$ & $\begin{array}{l}\text { 2HB Ticonderoga } \AA \text { brand pencil: Diameter: } 0.2 \text { inches, Shape }= \\
\text { Hexagonal flat edges }\end{array}$ \\
\hline Spice bottle (empty) & McCormick ${ }^{\circledR}$ brand Basil Leaves: Capacity $=0.62 \mathrm{Oz}$ \\
\hline Teaspoon (metal) & $\begin{array}{l}\text { Stainless steel teaspoon: Length }=515 / 16 \text { inches, Weight }=0.05 \\
\text { lbs. }\end{array}$ \\
\hline Writing paper pad & $\begin{array}{l}\text { Ampad }{ }^{\circledR} \text { brand gold fiber white ruled paper pad: Length }=113 / 4 \\
\text { inches, Width }=81 / 2 \text { inches. }\end{array}$ \\
\hline
\end{tabular}


Table 4.4. Characteristics of Participants with Neurological Conditions

\begin{tabular}{|c|c|c|c|c|c|c|c|c|c|c|}
\hline Participant & Diagnosis & Age & Sex & Education & $\begin{array}{l}\text { Affected } \\
\text { hand }\end{array}$ & $\begin{array}{l}\text { Current } \\
\text { Dominance }\end{array}$ & $M A M^{* *}$ & Fatigue*** & Depression*** & Pain*** $^{*}$ \\
\hline 1 & Stroke & 60 & $\mathrm{~F}$ & Bachelors & $\mathrm{R}$ & $\mathrm{R}$ & 90 & 45.8 & 55.7 & 55 \\
\hline 2 & Stroke & 70 & $\mathrm{~F}$ & Bachelors & $\mathrm{R}$ & $\mathrm{R}$ & 52.4 & 52.2 & 55.7 & 53.8 \\
\hline 3 & Stroke & 65 & M & $\mathrm{PhD}$ & $\mathrm{L}$ & $\mathrm{L}$ & 66.4 & 52.2 & 54.5 & 52.5 \\
\hline 4 & PD & 63 & $\mathrm{~F}$ & Bachelors & $\mathrm{R}$ & $\mathrm{R}$ & 67.6 & 47.6 & 62.1 & 52.5 \\
\hline 5 & PD & 61 & M & Bachelors & $\mathrm{R}$ & $\mathrm{R}$ & 63.3 & 53.7 & 53.3 & 41 \\
\hline 6 & PD & 62 & M & High School & $\mathrm{R}$ & $\mathrm{R}$ & 59.6 & 62 & 57.9 & 41 \\
\hline 7 & PD & 54 & M & Not reported & $\mathrm{L}$ & $\mathrm{L}$ & 53.9 & 50.8 & 56.8 & 56.1 \\
\hline 8 & Stroke & 52 & $\mathrm{~F}$ & High School & $\mathrm{L}$ & $\mathrm{R}^{*}$ & 55.5 & 50.8 & 62.1 & 41 \\
\hline 9 & Stroke & 46 & M & Bachelors & $\mathrm{L}$ & $\mathrm{L}$ & 49.3 & 43.9 & 64.1 & 41 \\
\hline 10 & Stroke & 60 & F & Masters & Both & Both & 59.6 & 55.1 & 70.3 & 56.1 \\
\hline 11 & MS & 50 & M & Bachelors & $\mathrm{L}$ & $\mathrm{R}$ & 68.9 & 53.7 & 53.3 & 41 \\
\hline 12 & MS & 52 & M & Bachelors & Both & $\mathrm{R}$ & 66.4 & 47.6 & 65.1 & 52.5 \\
\hline 13 & MS & 66 & $\mathrm{~F}$ & Bachelors & Both & $\mathrm{R}$ & 52 & 66.3 & 60 & 61.8 \\
\hline 14 & PD & 65 & $\mathrm{~F}$ & Masters & Both & $\mathrm{R}$ & 54.7 & 60.6 & 61.1 & 60.9 \\
\hline 15 & PD & 72 & M & High School & $\begin{array}{l}\text { Don't } \\
\text { know }\end{array}$ & $\mathrm{R}$ & 100 & 49.2 & 54.5 & 41 \\
\hline 16 & MS & 51 & M & Not reported & Both & $\mathrm{R}$ & 49.3 & 67.8 & 68.2 & 48.5 \\
\hline 17 & MS & 50 & $\mathrm{~F}$ & Bachelors & $\mathrm{L}$ & $\mathrm{R}$ & 60.5 & 41.9 & 53.3 & 41 \\
\hline 18 & TBI & 29 & M & $\begin{array}{l}\text { Less than } \\
\text { High School }\end{array}$ & Both & $\mathrm{R}$ & 47.8 & 55.1 & 70.3 & 59.1 \\
\hline 19 & MS & 51 & M & Bachelors & $\mathrm{R}$ & $\mathrm{R}$ & 68.9 & 53.7 & 53.3 & 41 \\
\hline 20 & MS & 51 & F & Bachelors & $\mathrm{L}$ & $\mathrm{R}$ & 60.5 & 41.9 & 53.3 & 41 \\
\hline 21 & Stroke & 32 & $\mathrm{~F}$ & Bachelors & $\mathrm{R}$ & $\mathrm{L}^{*}$ & 65.3 & 43.9 & 53.3 & 52.5 \\
\hline 22 & PD & 63 & M & High School & $\mathrm{R}$ & B & 59.6 & 62 & 57.9 & 41 \\
\hline 23 & Stroke & 47 & M & Bachelors & $\mathrm{L}$ & $\mathrm{R}$ & 49.3 & 43.9 & 64.1 & 41 \\
\hline 24 & PD & 62 & M & Bachelors & $\mathrm{L}$ & $\mathrm{R}$ & 63.3 & 53.7 & 53.3 & 41 \\
\hline 25 & TBI & 30 & M & $\begin{array}{l}\text { Less than } \\
\text { High School }\end{array}$ & Both & $\mathrm{R}$ & 47.8 & 55.1 & 70.3 & 59.1 \\
\hline 26 & TBI & 35 & M & Masters & $\mathrm{R}$ & $\mathrm{L}^{*}$ & 63.3 & 50.8 & 64.1 & 55 \\
\hline
\end{tabular}

Note: ${ }^{*}$ Changed dominance due to hand problems. ${ }^{* *}$ Higher scores indicate more of the trait and thus better hand function. ***Higher scores on these Patient Reported Outcome Measure Information System (PROMIS) measure indicate more of the trait, thus higher scores indicate more fatigue, more depression and more pain. MAM: Manual Ability Measure-20; Fatigue: PROMIS Fatigue short form 7a version 1.0; Depression: PROMIS Depression short form $8 \mathrm{~b}$ version 1.0; Pain: PROMIS Pain Interference short form $6 \mathrm{~b}$ version 1.0 
Table 4.5. New Items from Cognitive Interviews

\section{Items added from cognitive interviews}

2 liter coke bottle lifting and pouring

Button with a loop, pin for a badge

Cards and toys

Carpenter work

Chopping jalapenos

Close a paper clip

Close a safety pin

Collar tabs

Construction work

Cutting paper

Cutting tablets into half

Dishes

Drawing circles

Dump something from a big pot to a colander

Filing

Finger exerciser with rubber bands

Flipping an egg

Flipping pages of a book

Flossing

Folding papers

Hammer

Holding big phones

Holding book to read

Holding kids hand

Keyboard

Keys out of the pocket

Make piles of 10 coins

Measuring with measuring spoon

Moving big cans of tomato juice

Opening a can of beans

Opening good wrapped in plastic

Paper clips

Passing dishes or cups

Peel a kiwi
Peeling an apple

Picking up different coins

Picking up heavy items not in a bag

Picking up marbles

Picking up things from floor

Pin in a pin cushion

Placing objects at different locations

Pouring vanilla onto teaspoon

Puppets

Putting away groceries

Putting kids arms in coats

Putting toys away

Sample shirt buttoning

Scooping with a spoon

Screws and screwdriver use

Serving food

Slicing and dicing to the same size of vegetables

Small tablets

Stacking cans

Stapling

Threading the nut on a bolt

Toothpaste on toothbrush

Turning a pork chop frying on a pan

Tying a tie

Tying trash bags

Using a can opener

Using a needle

Using a spatula

Using a tape and mark a line

Using pliers

Using saw and cutting wood

Ziploc $₫$ bags

Zipping coats 


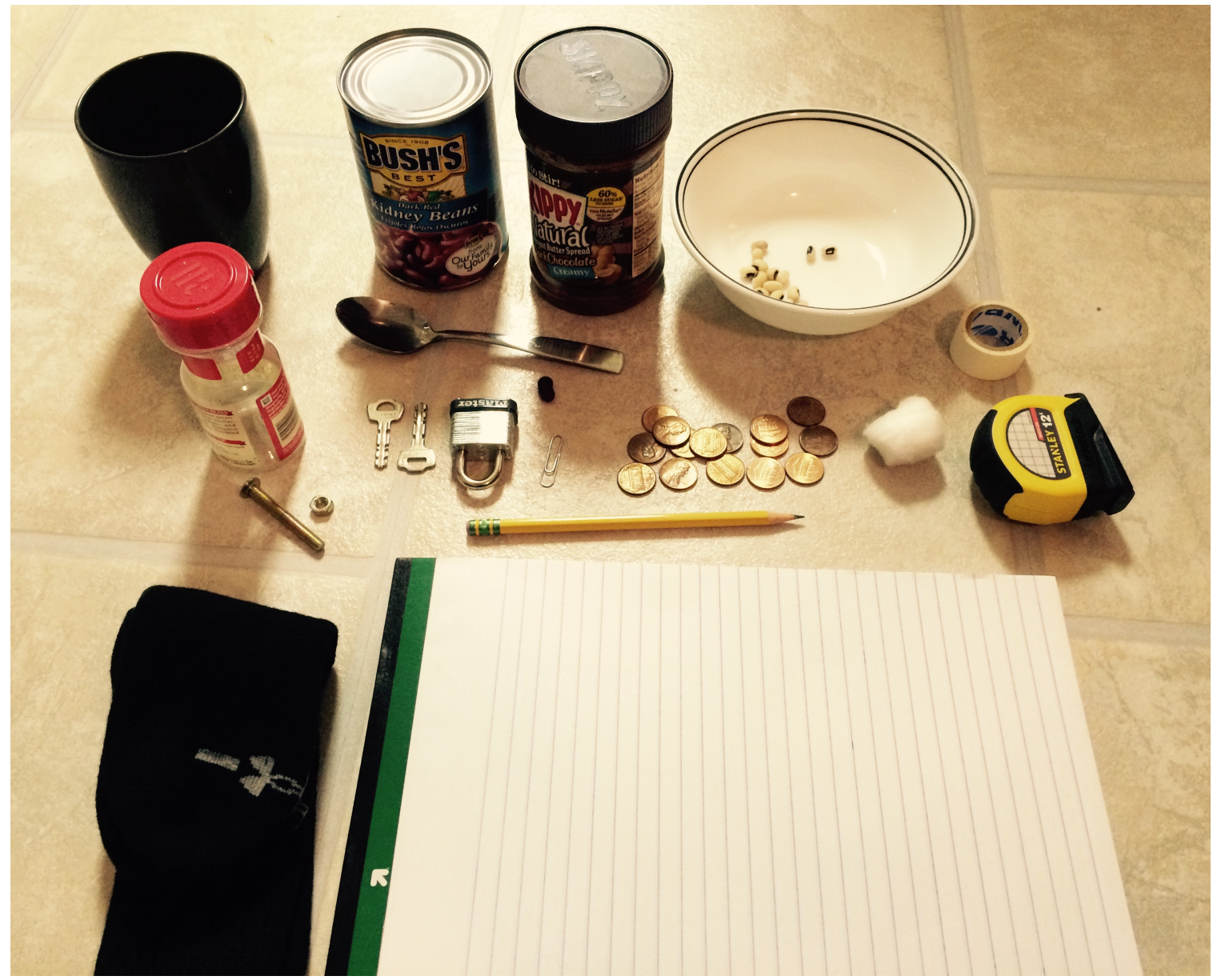

Figure 4.1. Materials Used for Performance-based Section of the Measure 


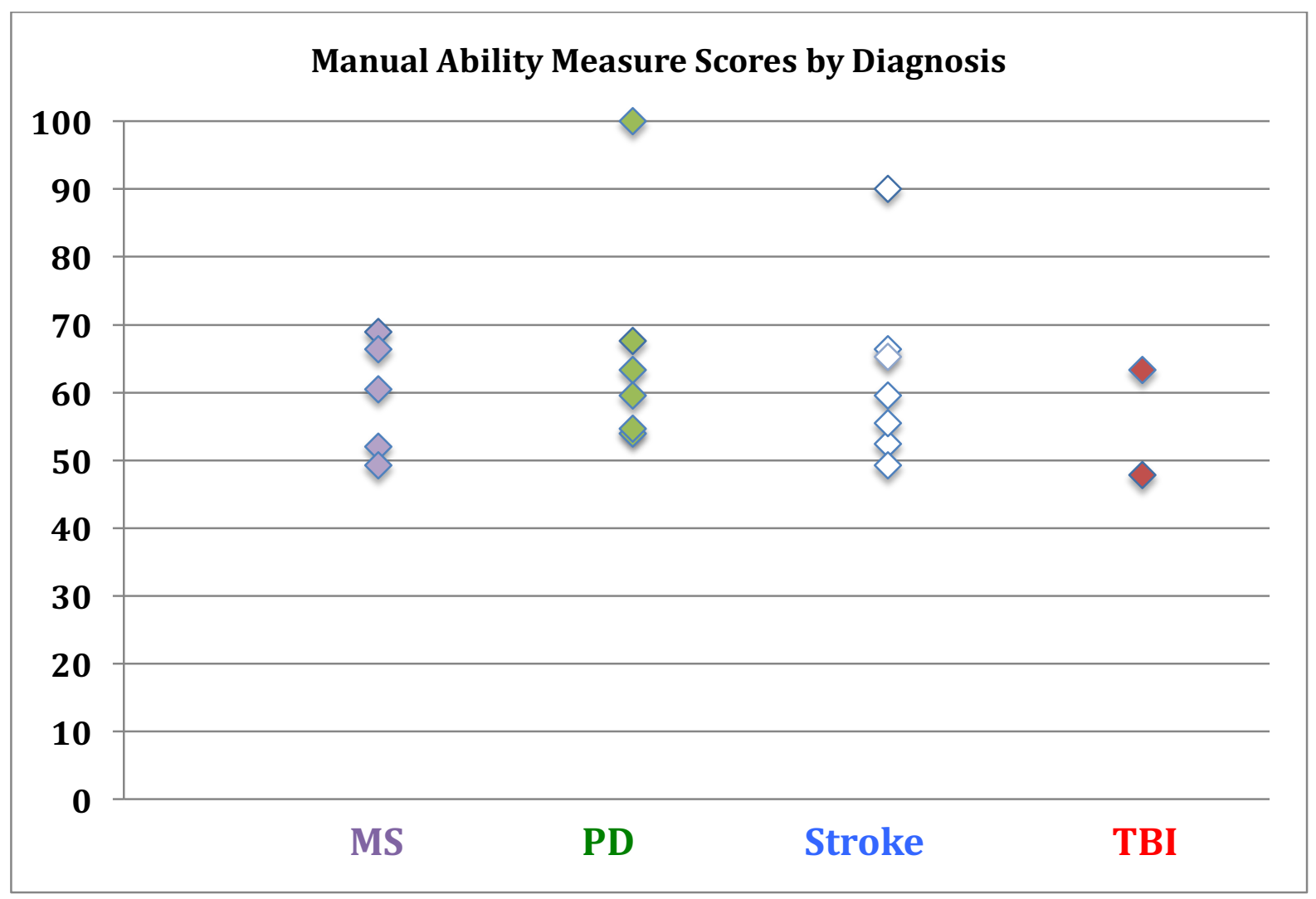

Figure 4.2. Manual Ability Measure Scores by Diagnosis 


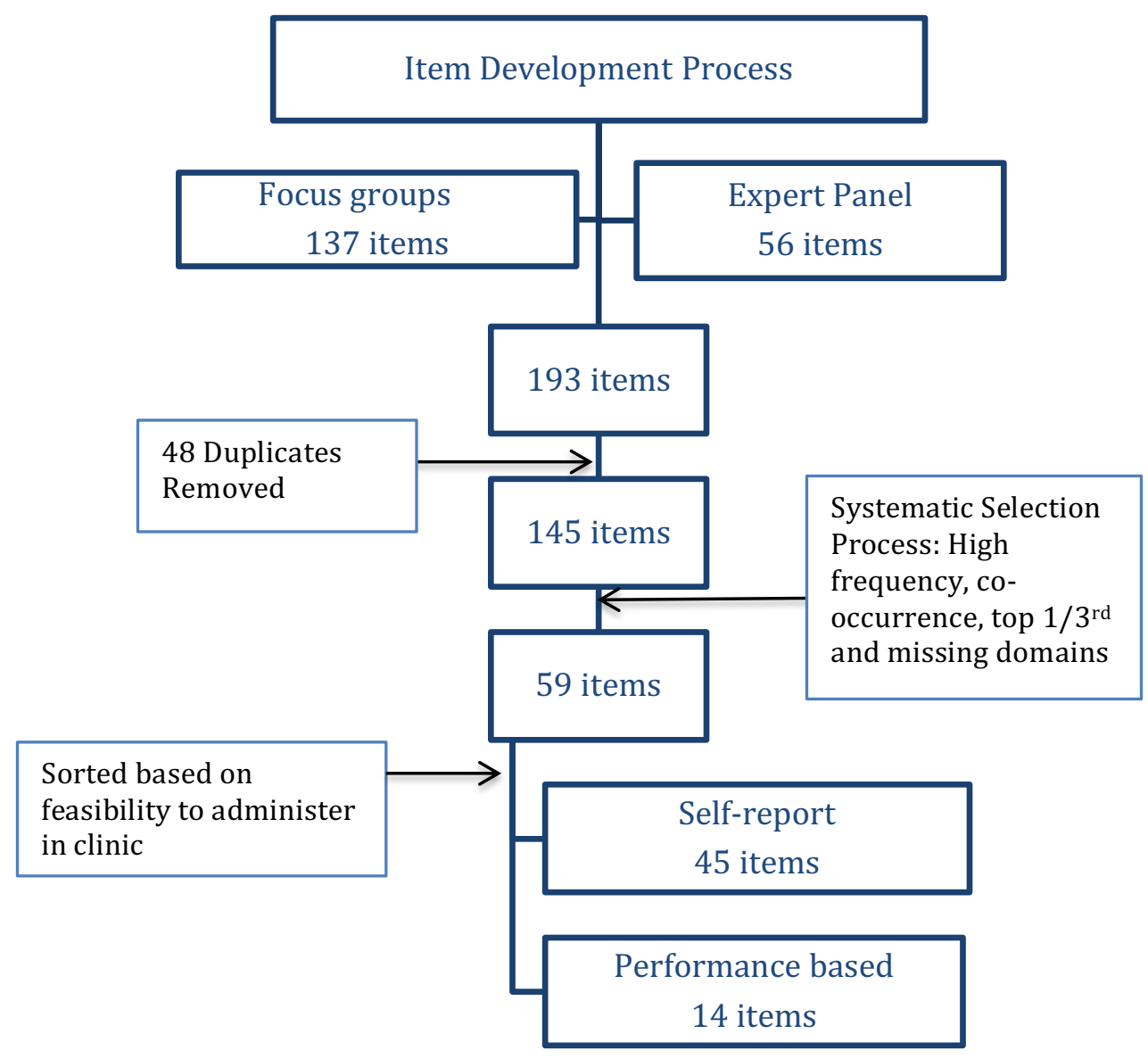

Figure 4.3. Item Development Process: Schematic Flow Diagram from Item Bank Development to Preliminary Selection of Items 


\section{Chapter 5: Discussion and Future Research}

This dissertation represents the first phase of development of a new measure. The purpose of the Hand and Arm Function Measure (HAFM) is to assess the quality and speed of task performance and self reported difficulty with body structure and function, activity and participation due hand and arm function issues in daily activities for people with neurological conditions. These aspects are assessed through self-report and performance-based sections of the measure. This chapter summarizes the initial development, provides an overarching discussion, and presents a plan for future development of the HAFM.

\section{Summary}

Chapter 1 provided an introduction to hand function in general and hand function issues in particular in neurological conditions. A brief overview of the different types of hand function tests and their limitations were presented. The recognized need for a new measure of hand and arm function, addresses two key components, self-reported hand function and quality and speed of task performance. None of the currently available measures assess both of these components and have limited evidence for validity of scores for use in neurological conditions. In order to develop the new measure the framework of evidence-centered design (ECD) was adopted. The reasoning behind the selection of this framework was its utility in developing self-reported and performance-based sections, its emphasis on analysis of the construct and strong connection of conceptual framework with psychometric testing decisions. This chapter described the five layers of ECD, the purpose of the research project, and objectives of the dissertation research study.

Chapter 2 included a literature review of upper extremity function classification, neural correlates and motor control basis, a systematic review of measures of upper extremity function and limitations of the currently available measures. The classification by Jones and Lederman 
(1993) was adopted for classifying the various functional aspects of hand and arm function. The model was selected for its focus on sensory motor function and was presented in detail in this chapter. The literature review on neural control provided a foundation for understanding the neurological control and motor control of hand and arm movements. The in-depth systematic review of hand and arm function tests presented in this chapter described 22 measures reported in the literature in the last ten years and discussed their limitations.

The development of the HAFM was informed by a panel of experts, focus groups, and cognitive interviews with people with neurological conditions. All provided useful insights into the definition of the construct, the structure of the measure, and the selection of items and responses. In Chapter 3, the construct of 'hand and arm function in daily activities' was explained with the help of a construct map and nomological network for neurological conditions. High level hand and arm function was defined as the ability of a person to participate in a wide range of daily activity tasks with the use of their hand and arm with optimal speed and quality, without experiencing interference by body structure and function problems and corresponds with higher scores on the HAFM. The International Classification of Functioning Disability and Health (ICF) framework that shaped this construct and the ECD framework that guided scale development are discussed in detail for their influences on the conceptualization of HAFM. The first phase of the research project involved engaging experts in defining the construct, item bank development, and item writing and modification. The item writing and modification were conducted after the focus groups with people with neurological conditions were conducted. Using a focus group format, the expert panel qualitative study was conducted from which eight key themes emerged. These themes informed the construct definition, item generation, shaped response format and scoring criteria, and identified the hand function issues faced by people with 
stroke, traumatic brain injury, multiple sclerosis and Parkinson disease and were described in detail in this Chapter 3.

For the next stage of the development of the HAFM, focus groups and cognitive interviews with people with neurological conditions were conducted and are presented in Chapter 4. The qualitative descriptive study results, their influence on the construct, item bank development, and item modification are discussed. The focus groups helped identify the aspects of hand function that were relevant to the participants with neurological conditions (i.e., sensation, strength, grasp, and fine hand use). The feedback from participants in each of the four diagnostic conditions helped to develop the item bank. The final item set was developed and preliminary items were administered to participants in the form of one-on-one cognitive interviews. Based on the cognitive interviews it was found that self-report and performancebased items were well tolerated by the participants. In addition, during the cognitive interviews, participants suggested modifications to the items, response options, and order of the items.

\section{Discussion}

The confidence in the results of this research emerge from triangulation. Triangulation is a strategy in qualitative research where the conclusions are drawn after data is gathered from different respondents, methodological approaches and frameworks (Golafshani, 2003). According to Golafshani (2003), there are four types of triangulation presented in literature, theoretical, methodological, data, and investigator. Theoretical triangulation relates to incorporating different theoretical frameworks. Methodological triangulation relates to having more than one methodological approach for collecting data. Data triangulation is concerned with data being gathered from more than one resource or participant group. Investigator triangulation occurs where two or more researchers take part in the data analysis. In this research study, 
theoretical triangulation was implemented with consideration of the ICF framework and Jones and Lederman's classification of hand function. Methodological triangulation included having more than one approach to explore the development of a new hand and arm function measure and included literature review, an expert panel, focus groups, and cognitive interviews. Data triangulation was addressed by collecting data from experts and participants with neurological conditions. Investigator triangulation was addressed by having with two researchers conduct the data analysis. These triangulation strategies have strengthened the qualitative approach utilized for developing the HAFM to inform face and content-related evidence for validity of the scores of the measure. The quantitative perspective of triangulation for item development is evident in the methodology of this research with a lack of deep discovery of the phenomenon of hand function problems. In recent years, similar methods have been utilized by other researchers in developing item banks (Morgan et al., 2014).

Limitations. This research study has limitations in the selection of one geographic location for data collection. It is also limited by small sample size, particularly for TBI. Some scale development studies employ large pool of participants (and include international participants (Annear et al., 2015). Although the current study has a small sample size, the participants were people with neurological conditions who are more difficult to recruit in clinical trials. This is also a limitation for applying the state-of-the-art Item Response Theory (IRT) methods since they require a large sample size for accurate statistical analysis. The fit statistics and differential item functioning cannot be conducted to establish construct-related evidence for validity of scores without the use of IRT based approaches. Although the Classical Test Theory (CTT) methods are not as robust as the IRT methods, they are the statistical method of choice for the current planning of the pilot study. 
Another limitation in the sample is the focus on four diagnostic conditions of stroke, traumatic brain injury (TBI), multiple sclerosis (MS), and Parkinson disease (PD). The measure, in its current form, is not designed for use with people with other types of neurological conditions. The studies of evidence for the reliability and validity of scores would need to be conducted in the future for use with people with these and other types of neurological conditions. The plethora of neurological conditions that could result in hand and arm function problems make it impossible to include all types of diagnoses in the study population. The two nonprogressive conditions of stroke and TBI and the two progressive conditions of MS and PD were assumed to be representative of the most commonly seen neurological conditions in the clinic that affect hand and arm function. Other conditions such as spinal cord injury (SCI) that can affect hand function were excluded from this study since the type of hand function problems in SCI are very unique to the condition (e.g., proximal more than distal issues in central cord syndrome), change considerably with the level of SCI (e.g., person with C6 tetraplegia with lost triceps function that is preserved in $\mathrm{C} 7$ tetraplegia), and involve special compensatory techniques (e.g., tenodesis in C6 tetraplegia). Moreover, specific measures such as the Capabilities of Upper Extremity (CUE) (Marino, Kern, Leiby, Schmidt-Read, \& Mulcahey, 2015) and Grasp and Release Test (GRT) (Mulcahey, Smith, \& Betz, 2004) have been developed in self-report (CUE) and performance-based (CUE and GRT) formats to measure hand and arm function in people with SCI. There are potentially many other neurological conditions, for which the measure would need to be validated. 


\section{Future Research}

The next step in the development of the HAFM is the pilot administration of the measure. The results of pilot administration can influence the construct, design of the measure, item design, and item bank.

HAFM Pilot Study. A brief blueprint for pilot administration is subsequently presented.

Purpose. The objective of the HAFM pilot study will be to investigate internal consistency, convergent, and discriminant evidence for validity of scores and to revise items as needed. The pilot study will involve administering the test items to people with neurological disorders such as stroke, TBI, MS, PD and people who do not have neurological disorders. The quantitative study will involve administering the HAFM and five other standardized measures of hand and arm function, and conducting preliminary psychometric analysis using classical test theory methods. The qualitative study will involve conducting 'think alouds' at the end of the complete administration of measure to get participant feedback on structure, flow, order, time and instructions related to entire measure and particular items revised in this iteration of the measure.

Participants. Forty adult participants, with eight participants in each group (stroke, TBI, MS, PD, and no neurological condition) will be recruited. Ideally, participants will represent a diverse range of age, ethnicity, and stage of the neurological condition (as appropriate) and have an equal representation of males and females. It is desired that these individuals have varying severities of hand and arm function from mild to severe and healthy adults. After human subjects approval, participants will be screened using a telephone interview. A hand function questionnaire such as the Stroke Impact Scale - Hand Domain (SIS-HD) (Duncan et al., 2002) 
will be used for screening. Participants will be enrolled in the study after inclusion criteria have been met through the screening and written informed consent is obtained.

Procedures. Each data session will consist of obtaining demographic information and administering a battery of assessments. Assessment will include the HAFM, the Montreal Cognitive Assessment Basic (Freitas, Simões, Marôco, Alves, \& Santana, 2012; Nasreddine et al., 2005), the National Institute of Health (NIH) Patient Reported Outcomes Information System (PROMIS) - measures for depression, fatigue and pain interference short forms (Gershon, Rothrock, Hanrahan, Bass, \& Cella, 2010), the Upper Extremity Neurological Quality of Life Short form (Cella et al., 2012), the Manual Ability Measure - 20 (Rallon \& Chen, 2008), the Quick DASH (Veehof, Sleegers, van Veldhoven, Schuurman, \& van Meeteren, 2002), the Nine Hole Peg Test (Oxford Grice et al., 2003), hand strength assessment for grip and pinch with the dynamometer (Peters et al., 2011), the Jebsen Hand Function Test (Stamm, Cieza, Machold, Smolen, \& Stucki, 2004) and the Timed Up And Go test (Mesquita et al., 2013). The self-report section of HAFM will be administered before the performance-based section.

Preliminary Data Analysis Plan. Classical item analysis statistics will be utilized for data analysis for this pilot study (Allen \& Yen, 2002). The statistics package Winsteps ${ }^{\circledR}$ (www.winsteps.com) will be used for analysis. This package was selected for its statistical tools that provide measurement related analysis. A multimethod multitrait matrix will be constructed as shown in Table 5.1. It is expected that the fine movements items on both sections of the HAFM will correlate highly and so will the gross movements items. To measure internal consistency, Cronbach's alpha will be computed for the test and acceptable scores are considered to be 0.70 or above and strong scores are considered to be 0.90 and above (McDowell, 2006). Convergent evidence for validity of scores can be established separately for each section of the 
measure since there is no other standard measure that has both sections in one measure. The relationship between the performance-based section of the measure, the Nine Hole Peg Test, the Jamar ${ }^{\circledR}$ dynamometer, and the Jebsen Hand Function Test; and the self-report section of the measure with the UE NeuroQoL Shortform, the Quick DASH and the MAM-20 will be explored. A strong correlation is expected between performance-based section and JHFT, and self-report section, the UE NeuroQoL Shortform, and the MAM-20. A moderate correlation is expected between performance-based section of the measure with NHPT and dynamometer, and the selfreport section with DASH. The discriminant evidence for validity of scores will be obtained by examining the relationship between HAFM and TUG (Timed Up and Go Test) will be examined with the hypothesis that there is expected to be a low correlation. A low correlation is expected versus no correlation because participants who struggle with mobility may be the more severely affected individuals; therefore, their hand and arm function may also be impacted. However, the correlation between TUG and the scores from the new measure should be lower than the correlations between scores from other hand function measures and scores from the new measure.

The construct-related evidence for validity of scores cannot be established by one study and has to be a combined result of multiple studies with varied samples (American Educational Research Association, 1999). In the recent literature related to hand and arm function assessment measures, as noted in the systematic review in Chapter 2, many studies in development of a measure are incomplete and do not address all psychometric properties, leaving the clinician with the choice of informal testing versus a poorly studied measure. Thus, the scope of this project is outlined to raise the standards of evidence to support validity and reliability of scores of the clinical measure of hand and arm function. 
Scope of research project. The overall goal of this project is to develop a reliable and valid measure of hand and arm function in people with neurological conditions that is psychometrically robust. Nine phases of the overall scope of this project were developed without considering the limitations of resources, participants and personnel and the research plan is outlined in Table 5.2. The item bank development with stakeholder feedback using focus groups and cognitive interviews is completed with evidence for content established in the current study. The results from the current study and the proposed pilot study will be used to help secure funding for larger studies. The item tryout study will recruit a representative sample $(\mathrm{N}=100)$ for standardized administration of the measure and run classical test theory (CTT) statistics to compute item level statistics. Item level statistics will help determine which items have a range of popularity (self-report section) or ability (performance-based section) and if all items contribute to their total score. The revised measure will then be ready for large-scale administration where a stratified sample $(\mathrm{N}=400)$ of participants across different settings and locations is expected for psychometric analysis. The state-of-art psychometric methods of item response theory (IRT) can be applied to this large sample to determine internal consistency, convergent and discriminant evidence for validity of scores and establishing cut off scores. Cut off scores will be determined using the contrasting groups method (Livingston \& Zieky, 1982). In this method, the therapists will be asked to determine severity of hand function deficits into categories of mild, moderate and severe. The cut-score range that corresponds to these categories will be computed. The healthy adults will provide the unimpaired category scores. Random samples can be pulled from this large sample for test retest and inter-rater evidence for reliability of scores, and convergent and discriminant evidence for validity of scores. Evidence to support the intended use of scores can then be investigated in a randomized controlled trial where the pre 
and post scores are compared for clinically meaningful change and minimal clinically important difference. A longitudinal follow-up of a subset of participants to gather evidence for unintended consequences of the measure is planned after the standardized measure is studied for clinical use.

\section{Conclusion}

To conclude, in the words of Robert DeVellis - a renowned measurement expert famous for his work with the NIH initiative on PROMIS and more than 30 years of experience in scale development, - "Even if a poor measure is the only one available, the costs of using it may be greater than any benefits attained." (DeVellis, 2012) (p. 10)

Clients and therapists should not have to spend valuable clinical time on measures that are poorly studied and likely of limited benefit to the client. Stakeholder engagement in the initial stages is key to developing the construct and the items that are valid for measuring hand and arm function in people with neurological conditions. Patient/client reported hand and arm function is valuable in measuring outcomes of rehabilitation. The HAFM is grounded in these concepts and is unique in its combination of self-reported and performance-based aspects included within a single measure.

This research has focused on the preliminary development of the HAFM with attention to rigor in designing a measure that reflects the needs of patients/clients and clinicians. This is a first step in the development of the HAFM. Much more additional work is necessary, from piloting to large-scale administration, for ongoing development to gather evidence for validation of scores of the measure. 
Table 5.1. Multitrait Multimethod Matrix with Expected Correlations

\begin{tabular}{|c|c|c|c|c|c|}
\hline & & \multicolumn{2}{|c|}{ HAFM Performance-based section } & \multicolumn{2}{|c|}{ HAFM Self-report section } \\
\hline & & Fine movements & Gross Movements & Fine movements & Gross Movements \\
\hline \multirow[t]{2}{*}{ HAFM Performance-based section } & Fine movements & 1.00 & Moderately High & High & Moderately High \\
\hline & Gross movements & Moderately High & 1.00 & Moderately High & High \\
\hline \multirow[t]{2}{*}{ HAFM Self-report section } & Fine movements & High & Moderately High & 1.00 & Moderately High \\
\hline & Gross movements & Moderately High & High & Moderately High & 1.00 \\
\hline
\end{tabular}


Table 5.2. Planning for Research to Support Interpretation and Use of Assessment Scores

\begin{tabular}{|c|c|c|c|c|c|}
\hline Purpose & Sample & Population & Analyses & Rationale & Status \\
\hline $\begin{array}{l}\text { Item bank } \\
\text { development } \\
\text { (Phase I) }\end{array}$ & $\begin{array}{l}\mathrm{N}=16 \\
\text { participants, } \\
\text { focus groups } \\
\mathrm{N}=8 \\
\text { participants, } \\
\text { cognitive } \\
\text { interviews } \\
\text { Purposeful } \\
\text { sampling }\end{array}$ & $\begin{array}{l}\text { Participants with } \\
\text { neurological } \\
\text { conditions of stroke, } \\
\text { TBI, MS, and PD. } \\
\text { Outpatient setting. } \\
\text { Equal gender } \\
\text { representation, } \\
\text { diversity among age } \\
\text { and ethnic background }\end{array}$ & $\begin{array}{l}\text { Qualitative analysis } \\
\text { - Ask participants to list difficulties with } \\
\text { hand and arm function in daily activities. } \\
\text { - Ask participants to respond to items and } \\
\text { write comments about clarity of items. } \\
\text { - Ask participants whether the tasks and } \\
\text { wording are universal and culturally } \\
\text { appropriate. } \\
\text { - Ask about parts that are unclear including } \\
\text { scoring }\end{array}$ & $\begin{array}{l}\text { - Gather lived experiences with hand } \\
\text { and arm problems of people with } \\
\text { neurological condition to build } \\
\text { content-related evidence for validity } \\
\text { of scores } \\
\text { - Pre-piloting of items for } \\
\text { appropriateness with the 'think aloud' } \\
\text { method. }\end{array}$ & Done \\
\hline $\begin{array}{l}\text { Content- } \\
\text { related } \\
\text { evidence for } \\
\text { validity for } \\
\text { evidence } \\
\text { (Phase I) }\end{array}$ & $\begin{array}{l}\mathrm{N}=4 \text { Expert } \\
\text { Panel } \\
\text { members } \\
\text { Purposeful } \\
\text { sampling }\end{array}$ & $\begin{array}{l}\text { Expert panel to } \\
\text { include } \\
\text { psychometrician and } \\
\text { therapists who are } \\
\text { subject matter experts } \\
\text { in neurological } \\
\text { rehabilitation in clinic, } \\
\text { teaching and research. }\end{array}$ & $\begin{array}{l}\text { Qualitative analysis } \\
\text { - Ask about the clinical needs, construct } \\
\text { definition } \\
\text { - One round of item bank development. } \\
\text { - Second round of item checking where the } \\
\text { experts are shown the items currently in the } \\
\text { measure and their opinion about items } \\
\text { related to the construct is reported }\end{array}$ & $\begin{array}{l}\text { - Expert panel can help us identify } \\
\text { items that are best suited for the } \\
\text { measure } \\
\text { - The second round is when the experts } \\
\text { are shown the items and their } \\
\text { comments are used to revise the items }\end{array}$ & Done \\
\hline $\begin{array}{l}\text { Pilot } \\
\text { administration } \\
\text { (Phase II) }\end{array}$ & $\begin{array}{l}\mathrm{N}=40 . \\
\text { Convenienc } \\
\text { e sample } \\
\text { with } 8 \\
\text { participants } \\
\text { in each of } \\
\text { the } 4 \\
\text { subgroups of } \\
\text { neurological } \\
\text { conditions } \\
\text { and healthy } \\
\text { adults }\end{array}$ & $\begin{array}{l}\text { Participants with } \\
\text { neurological } \\
\text { conditions of stroke, } \\
\text { TBI, MS, PD, and } \\
\text { healthy adults. Equal } \\
\text { gender representation, } \\
\text { diversity among age, } \\
\text { hand function and } \\
\text { ethnic background. } \\
\text { Clinic based } \\
\text { administration in } \\
\text { inpatient or outpatient } \\
\text { settings. }\end{array}$ & $\begin{array}{l}\text { Mixed method analyses } \\
\text { - Administer the HAFM and other } \\
\text { standardized measures. } \\
\text { - 'Think alouds' done at the end to comment } \\
\text { on the entire test, the order of tasks and the } \\
\text { time for entire test completion. } \\
\text { CTT methods used for: } \\
\text { - Internal consistency } \\
\text { - Convergent evidence for validity of scores } \\
\text { - Discriminant evidence for validity of scores } \\
\text { Revision of standardization protocols and } \\
\text { items based on results. }\end{array}$ & $\begin{array}{l}\text { - } \\
\text { - } \text { New and revised items from } \\
\text { Order effects for presentation of } \\
\text { self-report and performance-based } \\
\text { sections to be explored. } \\
\text { - } \quad \text { Norms for the timed tasks using data } \\
\text { from healthy adults and people with } \\
\text { neurological conditions is gathered } \\
\text { to categorize the timed tasks and } \\
\text { inform scoring. } \\
\text { - Secure funding for large scale } \\
\text { studies }\end{array}$ & Planned \\
\hline
\end{tabular}


Table 5.2. Planning for Research to Support Interpretation and Use of Assessment Scores (Continued)

\begin{tabular}{|c|c|c|c|c|c|}
\hline Purpose & Sample & Population & Analyses & Rationale & Status \\
\hline $\begin{array}{l}\text { Item tryouts } \\
\text { (Phase III) }\end{array}$ & $\begin{array}{l}\mathrm{N}=100 . \\
\text { Convenience } \\
\text { sample with } \\
20 \text { participants } \\
\text { in each } \\
\text { subgroup of } \\
\text { neurological } \\
\text { conditions and } \\
\text { healthy adults. }\end{array}$ & $\begin{array}{l}\text { Participants with neurological } \\
\text { conditions of stroke, TBI, MS, } \\
\text { PD, and healthy adults. } \\
\text { Participants from inpatient and } \\
\text { outpatient settings. One rural, } \\
\text { one sub-urban and one urban } \\
\text { setting is preferred. }\end{array}$ & $\begin{array}{l}\text { Quantitative analyses using CTT methods. } \\
\text { - Administer the HAFM } \\
\text { - Compute item statistics: } \\
\text { ○ Item means } \\
\text { ○ Item to total score correlations for the } \\
\text { two formats } \\
\circ \text { Item factor analyses } \\
\text { O Internal consistency } \\
\text { Convergent/ discriminant evidence for } \\
\text { validity of scores }\end{array}$ & $\begin{array}{l}\text { Check to see if items have } \\
\text { a range of popularity (self } \\
\text { report) or ability } \\
\text { (performance) with the } \\
\text { help of item means } \\
\text { All items contribute to their } \\
\text { total score. }\end{array}$ & Pending \\
\hline $\begin{array}{l}\text { Large scale } \\
\text { testing: } \\
\text { Standardization } \\
\text { and norming } \\
\text { (Phase IV) }\end{array}$ & $\begin{array}{l}\text { Sample of } 400 \\
\text { participants } \\
\text { across } \\
\text { different } \\
\text { locations. } \\
80 \text { participants } \\
\text { in each of the } \\
\text { sub- } \\
\text { populations. }\end{array}$ & $\begin{array}{l}\text { Stratified sample of participants } \\
\text { with: } \\
\text { Layer } 1 \text { - neurological } \\
\text { problems with hand function } \\
\text { impairment at different levels, } \\
\text { healthy adults. } \\
\text { Layer } 2 \text { - inpatient and } \\
\text { outpatient settings. } \\
\text { Layer } 3 \text { - One rural, one } \\
\text { suburban and one urban setting } \\
\text { desired. } \\
\text { Layer } 4 \text { - Equal males and } \\
\text { females are desired. } \\
\text { Layer } 5 \text { - Cultural mix is } \\
\text { desired. }\end{array}$ & $\begin{array}{l}\text { Quantitative analyses using IRT methods. } \\
\text { - Administer the HAFM and other } \\
\text { measures to representative sample. } \\
\text { - Compute subscale score statistics of: } \\
\text { Score means and standard deviations, } \\
\text { score frequency distributions, score for } \\
\text { different neurological conditions, Score } \\
\text { for males and females, factor analyses } \\
\text { for dimensions } \\
\text { - Alpha coefficient } \\
\text { - The therapists administering the HAFM } \\
\text { will be asked to determine mild, } \\
\text { moderate and severe hand function } \\
\text { impairment based on their judgment and } \\
\text { the contrasting groups method will be } \\
\text { used to determine cut scores. }\end{array}$ & $\begin{array}{l}\text { - Use IRT to create interval } \\
\text { scale } \\
\text { - Determination of clinically } \\
\text { meaningful change } \\
\text { - Determination of cut scores } \\
\text { for mild, moderate and } \\
\text { severe impairments based on } \\
\text { contrasting groups method. } \\
\text { - Dimensionality - check for } \\
\text { the two dimensions of gross } \\
\text { movements and fine } \\
\text { movements for performance- } \\
\text { based and symptoms and } \\
\text { daily activities for self-report } \\
\text { section. } \\
\text { Normative data for timed } \\
\text { tasks. }\end{array}$ & Pending \\
\hline
\end{tabular}


Table 5.2. Planning for Research to Support Interpretation and Use of Assessment Scores (Continued)

\begin{tabular}{|c|c|c|c|c|c|}
\hline Purpose & Sample & Population & Analyses & Rationale & Status \\
\hline $\begin{array}{l}\text { Test } \\
\text { Retest } \\
\text { Evidence } \\
\text { for } \\
\text { reliability } \\
\text { of scores } \\
\text { (Phase V) }\end{array}$ & $\begin{array}{l}\mathrm{N}=100 \text { patients with } \\
20 \text { in each sub- } \\
\text { population. A random } \\
\text { sample from the } \\
\text { standardization } \\
\text { sample may be pulled } \\
\text { out for evidence for } \\
\text { reliability of scores. }\end{array}$ & $\begin{array}{l}\text { Participants with } \\
\text { neurological conditions of } \\
\text { stroke, TBI, MS, PD, and } \\
\text { healthy adults. } \\
\text { Different levels of hand } \\
\text { function impairments in the } \\
\text { mild, moderate and severe } \\
\text { categories }\end{array}$ & $\begin{array}{l}\text { - Administer HAFM and other measures } \\
\text { twice within one week interval between } \\
\text { testing } \\
\text { - Compute correlations between scores } \\
\text { from first and second testing events. }\end{array}$ & $\begin{array}{l}\text { - Alpha coefficients provide } \\
\text { information if participants } \\
\text { respond consistently on items } \\
\text { within a subscale; } \\
\text { - Test-retest evidence for } \\
\text { reliability of scores informs if } \\
\text { participant scores are } \\
\text { consistent over time }\end{array}$ & Pending \\
\hline $\begin{array}{l}\text { Inter-rater } \\
\text { Evidence } \\
\text { for } \\
\text { reliability } \\
\text { of scores } \\
\text { (Phase VI) }\end{array}$ & $\begin{array}{l}\mathrm{N}=100 \text { patients with } \\
20 \text { in each sub- } \\
\text { population. } \\
\text { Applies to } \\
\text { performance-based } \\
\text { section of the } \\
\text { measure. }\end{array}$ & $\begin{array}{l}\text { Participants with } \\
\text { neurological conditions of } \\
\text { stroke, TBI, MS, PD, and } \\
\text { healthy adults. } \\
\text { Different levels of hand } \\
\text { function impairments in the } \\
\text { mild, moderate and severe } \\
\text { categories }\end{array}$ & $\begin{array}{l}\text { - Use video recordings from } \\
\text { standardization sample, shown to two } \\
\text { raters and their responses recorded. } \\
\text { - Two raters administer the HAFM and } \\
\text { their responses recorded. }\end{array}$ & $\begin{array}{l}\text { - The performance format of the } \\
\text { test should be consistent when } \\
\text { administered by two raters. } \\
\text { - High ICC between the two } \\
\text { raters are desired for the } \\
\text { measure when scoring the } \\
\text { responses. }\end{array}$ & Pending \\
\hline $\begin{array}{l}\text { Construct- } \\
\text { related } \\
\text { evidence } \\
\text { for } \\
\text { validity } \\
\text { (Phase } \\
\text { VII) }\end{array}$ & $\begin{array}{l}\mathrm{N}=100 \text { patients with } \\
20 \text { in each sub- } \\
\text { population. }\end{array}$ & $\begin{array}{l}\text { Participants with } \\
\text { neurological conditions of } \\
\text { stroke, TBI, MS, PD, and } \\
\text { healthy adults } \\
\text { By comparing healthy } \\
\text { adults and persons with } \\
\text { impaired hand function, } \\
\text { differences between them } \\
\text { can be explored. }\end{array}$ & $\begin{array}{l}\text { Quantitative analyses } \\
\text { - Administer HAFM and other measures } \\
\text { such as MAM-20 and JHFT. } \\
\text { No gold standard is available, and since } \\
\text { the HAFM self-report section is not a } \\
\text { precise reflection of the construct } \\
\text { presented in the MAM-20, only } \\
\text { moderate correlations are expected. } \\
\text { JHFT only measures time to completion } \\
\text { and in comparison with time } \\
\text { component of HAFM, moderate } \\
\text { correlations are expected here as well. }\end{array}$ & 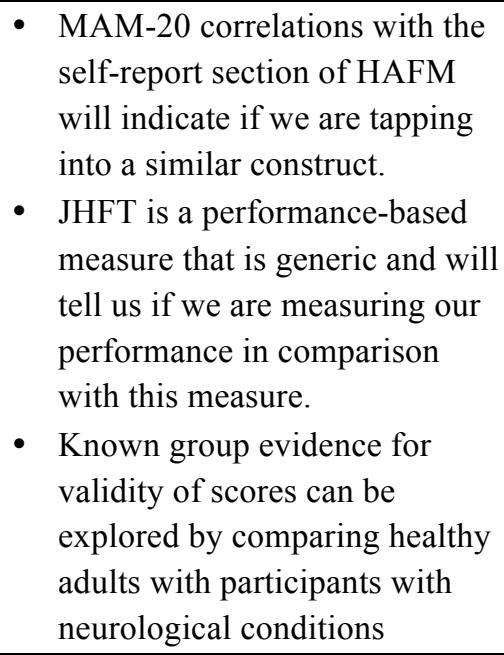 & Pending \\
\hline
\end{tabular}


Table 5.2. Planning for Research to Support Interpretation and Use of Assessment Scores (Continued)

\begin{tabular}{|c|c|c|c|c|c|}
\hline Purpose & Sample & Population & Analyses & Rationale & Status \\
\hline $\begin{array}{l}\text { Evidence to } \\
\text { support } \\
\text { intended use } \\
\text { of scores } \\
\text { (Phase VIII) }\end{array}$ & $\begin{array}{l}\mathrm{N}=120 \mathrm{Two} \\
\text { groups of } \\
\text { individuals with } \\
\text { neurological } \\
\text { conditions } \\
\text { randomized into } \\
\text { control }(\mathrm{N}=60) \\
\text { and treatment } \\
(\mathrm{N}=60) \\
\text { conditions. }\end{array}$ & $\begin{array}{l}\text { Participants } \\
\text { with } \\
\text { neurological } \\
\text { conditions of } \\
\text { stroke, TBI, } \\
\text { MS, PD }\end{array}$ & $\begin{array}{l}\text { Quasi experimental design of quantitative study } \\
\text { - HAFM administered to patients before and after an } \\
\text { intervention in the treatment group and the control } \\
\text { group. } \\
\text { - Therapists set the mild, moderate and severe categories } \\
\text { based on performance and these classifications are } \\
\text { matched with the cut scores obtained. } \\
\text { Also the pre-post scores are compared for clinically } \\
\text { meaningful change. }\end{array}$ & $\begin{array}{l}\text { To investigate if the test is } \\
\text { responsive to detect change with } \\
\text { intervention, specificity to not } \\
\text { change with no intervention and } \\
\text { ability to classify individuals } \\
\text { into categories of impairment } \\
\text { level as mild, moderate and } \\
\text { severe. }\end{array}$ & Pending \\
\hline $\begin{array}{l}\text { Evidence for } \\
\text { consequences } \\
\text { (Phase IX) }\end{array}$ & $\begin{array}{l}\mathrm{N}=100 \\
\text { patients with } 20 \\
\text { in each sub- } \\
\text { population. A } \\
\text { subset of people } \\
\text { who } \\
\text { participated in } \\
\text { the treatment } \\
\text { condition will } \\
\text { be followed } \\
\text { over time. }\end{array}$ & $\begin{array}{l}\text { Participants } \\
\text { with } \\
\text { neurological } \\
\text { conditions of } \\
\text { stroke, TBI, } \\
\text { MS, PD }\end{array}$ & $\begin{array}{l}\text { Qualitative study } \\
\text { - Follow the change in function over time in progressive } \\
\text { and non-progressive conditions for about } 2 \text { years. } \\
\text { Intended consequences: Did the therapists use the } \\
\text { results to plan interventions and do they relate to the } \\
\text { scores? Did the therapists continue to use the measure } \\
\text { to track progress? Assess how insurance } \\
\text { reimbursement was tied to the reporting of scores on } \\
\text { HAFM. } \\
\text { - Unintended consequences: Did the measure create } \\
\text { negative perceptions of self worth? Did the therapist's } \\
\text { bias in some form create an inaccurate scoring of the } \\
\text { measure? Construct irrelevant variance: Did another } \\
\text { factor such as depression affect the performance of the } \\
\text { individuals on the HAFM. } \\
\text { Ask the therapists about how they interpreted the test } \\
\text { scores and what did they use it for. Assess how } \\
\text { insurance reimbursement was tied to the reporting of } \\
\text { scores on this measure. }\end{array}$ & $\begin{array}{l}\text { Longitudinal perspective is } \\
\text { important to find out how } \\
\text { patients and therapists used the } \\
\text { measure and how the measure } \\
\text { affected their perspective } \\
\text { towards hand function or the test } \\
\text { effect. }\end{array}$ & Pending \\
\hline
\end{tabular}




\section{Appendix A. Definitions for Terms Used in this Study Related to Measurement Criteria}

\begin{tabular}{|c|c|}
\hline Term & Definition. \\
\hline Alpha & $\begin{array}{l}\text { Also known as the Cronbach's alpha. It is a generalized formula used to express } \\
\text { the internal consistency evidence for reliability of scores of a measure and varies } \\
\text { from } 0 \text { to } 1 \text {. }\end{array}$ \\
\hline Ceiling effects & $\begin{array}{l}\text { The effect in a measure where a high proportion of participants in a study have } \\
\text { maximum scores. }\end{array}$ \\
\hline $\begin{array}{l}\text { Claims and evidence } \\
\text { worksheets }\end{array}$ & $\begin{array}{l}\text { The claims made by an assessment tool and the evidence that supports that claim } \\
\text { are presented in the claims and evidence worksheets. (Mislevy et al., 2003) }\end{array}$ \\
\hline $\begin{array}{l}\text { Concurrent evidence for } \\
\text { validity of scores }\end{array}$ & $\begin{array}{l}\text { Evidence for validity of scores indicated by comparing scores on a measurement } \\
\text { with those obtained by applying alternative, equivalent measurement at the same } \\
\text { time. }\end{array}$ \\
\hline $\begin{array}{l}\text { Content-related evidence for } \\
\text { validity of scores }\end{array}$ & $\begin{array}{l}\text { The degree to which a measurement covers all aspects of the topic it purports to } \\
\text { measure. }\end{array}$ \\
\hline $\begin{array}{l}\text { Convergent evidence for } \\
\text { validity of scores }\end{array}$ & $\begin{array}{l}\text { The degree to which two or more instruments that purport to be measuring the } \\
\text { same topic agree with each other. }\end{array}$ \\
\hline Correlation & $\begin{array}{l}\text { The degree to which to which two or more sets of observations fit a linear } \\
\text { relationship. It lies between }-1 \text { to }+1 \text {. }\end{array}$ \\
\hline $\begin{array}{l}\text { Criterion-related evidence for } \\
\text { validity of scores }\end{array}$ & $\begin{array}{l}\text { The degree to which the results obtained using a measurement scale agree with a } \\
\text { criterion standard or indicator of true situation. }\end{array}$ \\
\hline $\begin{array}{l}\text { Cross cultural evidence for } \\
\text { validity of scores }\end{array}$ & $\begin{array}{l}\text { The degree to which two or more groups of culturally diverse groups agree with } \\
\text { each other. }\end{array}$ \\
\hline Design patterns & $\begin{array}{l}\text { Design patterns are tools to organize assessment arguments and are meant to guide } \\
\text { families of assessment tasks around aspects of proficiency (Mislevy et al., 2003). }\end{array}$ \\
\hline $\begin{array}{l}\text { Discriminant evidence for } \\
\text { validity of scores }\end{array}$ & $\begin{array}{l}\text { The extent to which scores on a measurement distinguish between individuals or } \\
\text { populations that would be expected to differ (e.g., people with or without stroke) }\end{array}$ \\
\hline Floor effects & $\begin{array}{l}\text { The lower limit to scores that cannot be reliably reported and people with low } \\
\text { level of the trait cannot be distinguished, is known as the floor effect. }\end{array}$ \\
\hline $\begin{array}{l}\text { Inter-rater evidence for } \\
\text { reliability of scores }\end{array}$ & $\begin{array}{l}\text { The extent to which the results obtained by different raters or interviewers using } \\
\text { the same measurement method will agree. }\end{array}$ \\
\hline Internal consistency & It is the degree to which all the items in a test, measure the same theme. \\
\hline $\begin{array}{l}\text { Intra-class correlation } \\
\text { coefficient }\end{array}$ & $\begin{array}{l}\text { The degree of agreement that records the average similarity of rater's actual scores } \\
\text { on the ratings being compared. }\end{array}$ \\
\hline $\begin{array}{l}\text { Intra-rater evidence for } \\
\text { reliability of scores }\end{array}$ & $\begin{array}{l}\text { The extent to which the results obtained by the same rater at two different time } \\
\text { points using the same measurement method will agree. }\end{array}$ \\
\hline $\begin{array}{l}\text { Minimal clinically important } \\
\text { difference }\end{array}$ & $\begin{array}{l}\text { The smallest change on a health outcome measure that would be considered } \\
\text { important by patient or clinician. }\end{array}$ \\
\hline Minimal detectable change & $\begin{array}{l}\text { The smallest change on a health outcome measure that corresponds to a noticeable } \\
\text { change in ability. }\end{array}$ \\
\hline $\begin{array}{l}\text { Predictive evidence for } \\
\text { validity of scores }\end{array}$ & The ability of the outcome of an instrument can predict a future outcome. \\
\hline $\begin{array}{l}\text { Evidence for reliability of } \\
\text { scores }\end{array}$ & The proportion of variance in a measurement that is not related to error. \\
\hline Evidence for responsiveness & The ability to detect change, critical for outcome measures. \\
\hline Standard error of measurement & The standard deviation of errors, the amount of error due to chance. \\
\hline $\begin{array}{l}\text { Test-retest evidence for } \\
\text { reliability of scores }\end{array}$ & $\begin{array}{l}\text { The repeatability of measurement evaluated in terms of agreement between the } \\
\text { measure administered at two different time points. }\end{array}$ \\
\hline Toulmin diagrams & $\begin{array}{l}\text { Toulmin diagrams are examples of tools for organizing assessment arguments at a } \\
\text { narrative level (Mislevy et al., 2003). }\end{array}$ \\
\hline Evidence for validity of scores & The extent to which a measure is measuring what it was intended to measure. \\
\hline
\end{tabular}

Note: Definitions adapted from the book 'Health Measurements'(McDowell, 2006) except when indicated otherwise 


\section{Appendix B. Studies Identified in the Systematic Review for the Self-report Measures}

\begin{tabular}{|c|c|}
\hline Measure & Studies Identified in the Systematic Review \\
\hline $\begin{array}{l}\text { Disabilities of Arm } \\
\text { Shoulder and Hand }\end{array}$ & $\begin{array}{l}3 \text { studies } \\
\text { (Cano, Barrett, Zajicek, \& Hobart, 2011; Hijmans, Hale, Satherley, McMillan, \& King, } \\
\text { 2011; Kraft et al., 2014) }\end{array}$ \\
\hline ABILHAND & $\begin{array}{l}2 \text { studies } \\
\text { (Alt Murphy, Willén, \& Sunnerhagen, 2012; Kraft et al., } \\
\text { 2014) }\end{array}$ \\
\hline Motor Activity Log & $\begin{array}{l}17 \text { studies } \\
\text { (Bowman et al., 2006; Brogårdh \& Lexell, 2010; Brunner, Skouen, \& Strand, 2012; } \\
\text { Celik et al., 2010; Dahl et al., 2008; Hammer \& Lindmark, 2010; Lamers, Kerkhofs, et } \\
\text { al., 2013; Lamers, Timmermans, et al., 2013; Lin, Wu, Wei, Lee, \& Liu, 2007; Keh- } \\
\text { chung Lin, Chang, Wu, \& Chen, 2009; Lin, Huang, Hsieh, \& Wu, 2009; Pang, Harris, \& } \\
\text { Eng, 2006; Taub et al., 2013; Timmermans et al., 2014; van Delden, Peper, Beek, \& } \\
\text { Kwakkel, 2013; Steven L. Wolf et al., 2006) }\end{array}$ \\
\hline $\begin{array}{l}\text { Stroke Impact Scale - } \\
\text { Hand Domain }\end{array}$ & $\begin{array}{l}11 \text { studies } \\
\text { (Boyne, Dunning, Levine, Hermann, \& Page, 2010; Combs, Kelly, Barton, Ivaska, \& } \\
\text { Nowak, 2010; Dahl et al., 2008; Gurcay, Bal, \& Cakci, 2009; Huang, Wu, Hsieh, \& Lin, } \\
\text { 2010; Keh-chung Lin, Chang, et al., 2009; Keh-chung Lin, Wu, et al., 2009; Page et al., } \\
\text { 2011; L. Shaw et al., 2010; van Delden, Peper, Beek, et al., 2013; Winstein et al., 2013) }\end{array}$ \\
\hline Manual Ability Measure & $\begin{array}{l}1 \text { study } \\
\text { (Kraft et al., 2014) }\end{array}$ \\
\hline $\begin{array}{l}\text { Neurological Quality of } \\
\text { Life - Upper Extremity }\end{array}$ & $\begin{array}{l}1 \text { study } \\
\text { (Kraft et al., 2014) }\end{array}$ \\
\hline $\begin{array}{l}\text { Patient Reported } \\
\text { Outcome Measure } \\
\text { Information System - } \\
\text { Upper Extremity }\end{array}$ & $\begin{array}{l}2 \text { studies } \\
\text { (Kraft et al., 2014; Lamers, Kerkhofs, et al., 2013) }\end{array}$ \\
\hline
\end{tabular}


Appendix C. Studies Identified in the Systematic Review for the Performance-based Measures

\begin{tabular}{|c|c|}
\hline Measure & Studies Identified in the Systematic Review \\
\hline $\begin{array}{l}\text { Jebsen Hand } \\
\text { Function Test }\end{array}$ & $\begin{array}{l}4 \text { studies } \\
\text { (Adamovich et al., 2009; Celik et al., 2010; Kraft et al., 2014; Wu, Seo, \& Cohen, 2006) }\end{array}$ \\
\hline $\begin{array}{l}\text { Rivermead } \\
\text { Movement } \\
\text { Assessment - Upper } \\
\text { Extremity }\end{array}$ & $\begin{array}{l}1 \text { study } \\
\text { (J. H. Morris et al., 2008) }\end{array}$ \\
\hline $\begin{array}{l}\text { Motricity Index - } \\
\text { Upper Extremity } \\
\text { Section }\end{array}$ & $\begin{array}{l}\text { studies } \\
\text { (Lamers, Kerkhofs, et al., 2013; Lamers, Timmermans, et al., 2013; L. Shaw et al., 2010) }\end{array}$ \\
\hline $\begin{array}{l}\text { Action Research } \\
\text { Arm Test }\end{array}$ & $\begin{array}{l}34 \text { studies } \\
\text { (Alt Murphy et al., 2012; Au-Yeung \& Hui-Chan, 2014; S. R. Barreca et al., 2005; Boyne et } \\
\text { al., 2010; Brunner et al., 2012; Burns, Burridge, \& Pickering, 2007; Cano et al., 2011; Celik } \\
\text { et al., 2010; H. Chen, Lin, Wu, \& Chen, 2012; Chuang, Wu, \& Lin, 2012; Hammer \& } \\
\text { Lindmark, 2010; Hughes et al., 2009; Ietswaart et al., 2011; Kraft et al., 2014; Lamers, } \\
\text { Kerkhofs, et al., 2013; Lamers, Timmermans, et al., 2013; J.-H. Lin, Hsu, et al., 2009; } \\
\text { McDonnell, Hillier, Ridding, \& Miles, 2006; J. H. Morris et al., 2008; J. H. Morris \& Van } \\
\text { Wijck, 2012; Page, Hade, \& Persch, 2015; Page, Levine, \& Hade, 2012; Pomeroy et al., } \\
\text { 2014; Rabadi \& Rabadi, 2006; Rosewilliam, Malhotra, Roffe, Jones, \& Pandyan, 2012; Seo, } \\
\text { Fischer, Bogey, Rymer, \& Kamper, 2011; L. Shaw et al., 2010; Timmermans et al., 2014; } \\
\text { Turk et al., 2008; Urbin, Waddell, \& Lang, 2015; van Delden, Peper, Nienhuys, et al., 2013; } \\
\text { van Delden, Peper, Beek, et al., 2013; Wu et al., 2006; Yozbatiran et al., 2008) }\end{array}$ \\
\hline $\begin{array}{l}\text { Fugl-Meyer } \\
\text { Assessment - Upper } \\
\text { Extremity }\end{array}$ & $\begin{array}{l}34 \text { studies } \\
\text { (Alt Murphy et al., 2012; Arya \& Pandian, 2013; Bartolo et al., 2014; Bowman et al., 2006; } \\
\text { Celik et al., 2010; Chae et al., 2009; Chan, Tong, \& Chung, 2009; Daly et al., 2005; Hammer } \\
\text { \& Lindmark, 2010; Hesse et al., 2005; Hijmans et al., 2011; Hughes et al., 2009; Knutson et } \\
\text { al., 2012; Krebs et al., 2008; Lamers, Kerkhofs, et al., 2013; Lamers, Timmermans, et al., } \\
\text { 2013; Levy et al., 2008; J.-H. Lin, Hsu, et al., 2009; Keh-chung Lin, Chang, et al., 2009; } \\
\text { Keh-Chung Lin, Huang, et al., 2009; Keh-chung Lin, Wu, et al., 2009; Masiero, Celia, } \\
\text { Rosati, \& Armani, 2007; McDonnell et al., 2006; Ohn et al., 2013; Page et al., 2011, 2015, } \\
\text { 2012; Pang et al., 2006; Rabadi \& Rabadi, 2006; Reinkensmeyer et al., 2012; Rosati, Gallina, } \\
\text { \& Masiero, 2007; Sullivan, Hurley, \& Hedman, 2012; Taub et al., 2013; Turk et al., 2008) }\end{array}$ \\
\hline $\begin{array}{l}\text { Function Test of } \\
\text { Hemiplegic Upper } \\
\text { Extremity }\end{array}$ & 1 study (Chan et al., 2009) \\
\hline $\begin{array}{l}\text { Box and Blocks } \\
\text { Test }\end{array}$ & $\begin{array}{l}6 \text { studies } \\
\text { (Higgins et al., 2006; Knutson et al., 2012; Kraft et al., 2014; Milot et al., 2014; } \\
\text { Reinkensmeyer et al., 2012; Seo et al., 2011) }\end{array}$ \\
\hline Nine Hole Peg Test & $\begin{array}{l}7 \text { studies } \\
\text { (Brunner et al., 2012; Higgins et al., 2006; Kraft et al., 2014; Lamers, Kerkhofs, et al., 2013; } \\
\text { J. H. Morris et al., 2008; J. H. Morris \& Van Wijck, 2012; Polman \& Rudick, 2010; L. Shaw } \\
\text { et al., 2010) }\end{array}$ \\
\hline $\begin{array}{l}\text { Function Test of } \\
\text { Hemiplegic Upper } \\
\text { Extremity }\end{array}$ & 1 study (Chan et al., 2009) \\
\hline $\begin{array}{l}\text { Box and Blocks } \\
\text { Test }\end{array}$ & $\begin{array}{l}6 \text { studies } \\
\text { (Higgins et al., 2006; Knutson et al., 2012; Kraft et al., 2014; Milot et al., 2014; } \\
\text { Reinkensmeyer et al., 2012; Seo et al., 2011) }\end{array}$ \\
\hline
\end{tabular}




\section{Appendix C. Studies Identified in the Systematic Review for the Performance-based Measures (Continued)}

\begin{tabular}{|c|c|}
\hline Hand Function Measure & Studies Identified in the Systematic Review \\
\hline $\begin{array}{l}\text { Function Test of Hemiplegic } \\
\text { Upper Extremity }\end{array}$ & 1 study (Chan et al., 2009) \\
\hline Box and Blocks Test & $\begin{array}{l}6 \text { studies } \\
\text { (Higgins et al., 2006; Knutson et al., 2012; Kraft et al., 2014; Milot et al., 2014; } \\
\text { Reinkensmeyer et al., 2012; Seo et al., 2011) }\end{array}$ \\
\hline Nine Hole Peg Test & $\begin{array}{l}7 \text { studies } \\
\text { (Brunner et al., 2012; Higgins et al., 2006; Kraft et al., 2014; Lamers, Kerkhofs, et } \\
\text { al., 2013; J. H. Morris et al., 2008; J. H. Morris \& Van Wijck, 2012; Polman \& } \\
\text { Rudick, 2010; L. Shaw et al., 2010) }\end{array}$ \\
\hline Motor Assessment Scale & $\begin{array}{l}4 \text { studies } \\
\text { (Brauer, Hayward, Carson, Cresswell, \& Barker, 2013; Brogårdh \& Lexell, 2010; } \\
\text { Hammer \& Lindmark, 2010; Sabari et al., 2005) }\end{array}$ \\
\hline Sollerman Hand Function Test & $\begin{array}{l}1 \text { study } \\
\text { (Brogårdh \& Lexell, 2010) }\end{array}$ \\
\hline Arm Motor Ability Test & $\begin{array}{l}6 \text { studies } \\
\text { (Chae et al., 2009; Daly et al., 2005; Daly \& Ruff, 2007; Knutson et al., 2012; } \\
\text { Levy et al., 2008; Sullivan et al., 2012) }\end{array}$ \\
\hline Wolf Motor Function Test & $\begin{array}{l}11 \text { studies } \\
\text { (Adamovich et al., 2009; Borstad et al., 2013; Bowman et al., 2006; Combs et al., } \\
\text { 2010; Dahl et al., 2008; Hijmans et al., 2011; J.-H. Lin, Hsu, et al., 2009; Pang et } \\
\text { al., 2006; Sawaki et al., 2008; Winstein et al., 2013; Steven L. Wolf et al., 2006) }\end{array}$ \\
\hline $\begin{array}{l}\text { Test Evaluant la Performance } \\
\text { des Membres supérieurs des } \\
\text { Personnes âgées }\end{array}$ & $\begin{array}{l}2 \text { studies } \\
\text { (Higgins et al., 2006; Kraft et al., 2014) }\end{array}$ \\
\hline $\begin{array}{l}\text { Stroke Rehab Evaluation } \\
\text { Assessment of Movement }\end{array}$ & $\begin{array}{l}2 \text { studies } \\
\text { (Higgins et al., 2006; J.-H. Lin, Hsu, et al., 2009) }\end{array}$ \\
\hline $\begin{array}{l}\text { Chedokee Arm and Hand } \\
\text { Inventory }\end{array}$ & $\begin{array}{l}6 \text { studies } \\
\text { (Abdullah, Tarry, Lambert, Barreca, \& Allen, 2011; S. R. Barreca et al., 2005; } \\
\text { Gustafsson, Turpin, \& Dorman, 2010; Harris, Eng, Miller, \& Dawson, 2009; } \\
\text { Schuster-Amft et al., 2014; Wu et al., 2006) }\end{array}$ \\
\hline
\end{tabular}




\section{Appendix D. Item Bank with Descriptions}

\begin{tabular}{|c|c|}
\hline Item & Description \\
\hline Carrying: Carry groceries & Able to carry groceries and bring it home or take it to the car. \\
\hline Carrying: Coffee cup & Able to carry a coffee cup and take it with you. \\
\hline Carrying: Shoulder bag & Able to carry a bag on the shoulder \\
\hline Carrying: Wine glass & Able to carry a wine glass in the affected hand. \\
\hline Communication: Clapping & Able to clap hands \\
\hline Communication: Shaking hand & Able to shake hands with another person \\
\hline Communication: Writing & $\begin{array}{l}\text { Able to use a writing device like pen or pencil and sign name, make lists in } \\
\text { a legible handwriting within a reasonable amount of time. }\end{array}$ \\
\hline IADL: Childcare tasks & Able to hold and carry a child, play with the child. \\
\hline IADL: Drive thru services & Able to use the drive thru services (e.g. fast food, parking tickets). \\
\hline IADL: Grocery: General & Able to do general grocery related tasks like going to grocery store. \\
\hline $\begin{array}{l}\text { IADL: Grocery: Opening thin plastic } \\
\text { bags at store }\end{array}$ & $\begin{array}{l}\text { Able to open thin plastic bags at the store to put grocery items in it like } \\
\text { vegetables. }\end{array}$ \\
\hline $\begin{array}{l}\text { IADL: Grocery: Sign at cashier's } \\
\text { counter }\end{array}$ & $\begin{array}{l}\text { Able to sign your name on the receipt or on the electronic pad at the } \\
\text { cashier's counter. }\end{array}$ \\
\hline IADL: Hanging clothes on line & Able to hang clothes on a clothes line \\
\hline IADL: Housekeeping: General & Able to do general housekeeping tasks \\
\hline IADL: Housekeeping: Mopping & Able to hold a mop with or without a handle and mop the floor \\
\hline IADL: Housekeeping: Pet care & Able to take care of the pet, stroking the pet, caring for the dog or cat. \\
\hline IADL: Housekeeping: Vacuum & Able to vacuum the carpet. \\
\hline IADL: Kitchen tasks: Cooking & Able to cook a meal. \\
\hline $\begin{array}{l}\text { IADL: Kitchen tasks: Cracking an } \\
\text { egg }\end{array}$ & $\begin{array}{l}\text { Able to break an egg without spilling or dropping the shell, able to get the } \\
\text { shell out if dropped. }\end{array}$ \\
\hline IADL: Kitchen tasks: Creamer cups & Able to open creamer cups that need small pinch and strength to pull \\
\hline IADL: Kitchen tasks: Cutting & Able to cut up food in the kitchen like vegetables, meat. \\
\hline $\begin{array}{l}\text { IADL: Kitchen tasks: Getting plastic } \\
\text { off the cheese slices }\end{array}$ & Able to get the plastic off the cheese slices \\
\hline IADL: Kitchen tasks: Kneading & Able to knead the dough. \\
\hline IADL: Kitchen tasks: Knife & Able to use a knife, cutting knife or pocket knife. \\
\hline IADL: Kitchen tasks: Microwave & Able to use the microwave. \\
\hline IADL: Kitchen tasks: Peeling & Able to hold the vegetable in one hand use a peeler with the other. \\
\hline $\begin{array}{l}\text { IADL: Kitchen tasks: Pick up pot on } \\
\text { stove }\end{array}$ & Able to pick up a pot with hot contents from the stove. \\
\hline IADL: Kitchen tasks: Plates & Able to hold and carry plates to and from the kitchen \\
\hline $\begin{array}{l}\text { IADL: Kitchen tasks: Pour } \\
\text { water/soups/milk }\end{array}$ & Able to pour water, soup, milk \\
\hline IADL: Kitchen tasks: Stirring & Able to stir hot items, particularly holding the container and stirring \\
\hline IADL: Laundry, folding clothes & Able to fold clothes, using the washer/dryer \\
\hline IADL: Laundry: Towel - folding & Able to fold a towel \\
\hline IADL: Pushing - wheelchair, cart & Able to push the grocery cart, wheelchair for someone else with your hands \\
\hline IADL: Washing the car & Able to wash own car \\
\hline IADL: Work: General & Issues with work \\
\hline IADL: Work: Return to work & Issues with return to work \\
\hline IADL: Work: Type of work & Different types of paid and unpaid work \\
\hline IADL: Work: Vocational Rehab & Able to get back to work using vocational rehabilitation services \\
\hline Movement: Holding steady & Able to hold objects steady \\
\hline Movements: Lifting & Able to lift items \\
\hline Movements: Opening/Closing hand & Able to open and close the hand \\
\hline Movements: Pick up & Able to pick up items \\
\hline
\end{tabular}




\section{Appendix D. Item Bank with Descriptions (Continued)}

\begin{tabular}{|c|c|}
\hline Item & Description \\
\hline Movements: Placing on table & Able to place hand on table \\
\hline Movements: Tap on table & Able to tap on the table \\
\hline $\begin{array}{l}\text { Object handling: Bags, purses, } \\
\text { backpack }\end{array}$ & Manipulating bags, purses, backpacks \\
\hline Object handling: Ball & Able to catch or throw ball \\
\hline Object handling: Bottle & Able to pick up, close and open a bottle and handle various caps \\
\hline Object handling: Cans & Able to open, close, hold and pick up cans \\
\hline $\begin{array}{l}\text { Object handling: Childproof bottle } \\
\text { for medications }\end{array}$ & Able to open and close childproof bottle for medications \\
\hline Object handling: Coins & Able to gather coins, count them in hand \\
\hline $\begin{array}{l}\text { Object handling: Credit cards } \\
\text { handling }\end{array}$ & Able to handle credit cards during shopping \\
\hline Object handling: Cup & Able to pick up and pour into a cup \\
\hline Object handling: Doors & Able to open and close doors. \\
\hline Object handling: Filing papers & Able to file papers \\
\hline Object handling: Hammer/Nail & Able to hammer a nail \\
\hline Object handling: Jar opening & Able to open and close a jar \\
\hline Object handling: Keys & Able to use keys to open doors, locks \\
\hline $\begin{array}{l}\text { Object handling: Kitchen tasks: } \\
\text { Flipping }\end{array}$ & Able to flip like an omelet when cooking to get the other side cooked. \\
\hline Object handling: Lids & Able to open and close lids, particularly the tight ones \\
\hline Object handling: Light switches & Able to use light switches in the home and outside \\
\hline Object handling: Locks & Able to handle locks \\
\hline Object handling: Mail & Able to take the mail, carry it, deliver it. \\
\hline Object handling: Milk & $\begin{array}{l}\text { Able to open and close, milk carton, milk can, milk gallon, milk half gallon, } \\
\text { includes carrying gallon of milk }\end{array}$ \\
\hline Object handling: Nuts and bolts & Able to tighten nuts and bolts \\
\hline $\begin{array}{l}\text { Object handling: Opening pocket } \\
\text { knife }\end{array}$ & Able to use a pocket knife \\
\hline Object handling: Paper money & Able to hold paper money, fold it, stack it. Put it in and out of wallet/ purse. \\
\hline Object handling: Peanut butter jar & Able to open a peanut butter jar \\
\hline Object handling: Pegboard & Able to put pegs on a pegboard \\
\hline Object handling: Pen & Able to use a pen for writing \\
\hline Object handling: Pencil & Able to use a pencil for writing \\
\hline $\begin{array}{l}\text { Object handling: Picking golf balls } \\
\text { from jar }\end{array}$ & Able to pick golf balls and put them in a jar, and take them out. \\
\hline Object handling: Pictures/Photos & Able to take a picture using camera or phone \\
\hline Object handling: Pills & Able to pick up pills, they drop \\
\hline Object handling: Pins & Able to pick up pins, hair pins, clips \\
\hline Object handling: Remote & Able to use a TV remote \\
\hline Object handling: Rubber band & Able to use a rubber band to tie items, tie hair \\
\hline Object handling: Scissors & Able to use scissors \\
\hline Object handling: Screw - turning & Able to turn a screw with a screwdriver \\
\hline $\begin{array}{l}\text { Object handling: Screwdriver into } \\
\text { screw }\end{array}$ & Able to place a screwdriver into a screw (before turning it) \\
\hline Object handling: Seatbelt use & Able to put on the seatbelt \\
\hline Object handling: Soap & Able to handle soap, slips off \\
\hline Object handling: Socks & Able to put on socks \\
\hline Object handling: Spoon & Able to use spoon, fork for eating, stirring. \\
\hline
\end{tabular}




\section{Appendix D. Item Bank with Descriptions (Continued)}

\begin{tabular}{|c|c|}
\hline Item & Description \\
\hline Object handling: Straw & Able to use a straw, place it and drink from it. \\
\hline $\begin{array}{l}\text { Object handling: Technology related: } \\
\text { Computer related tasks }\end{array}$ & $\begin{array}{l}\text { Able to do computer related tasks such as browsing the internet, finding } \\
\text { information, Able to drag and drop objects on the screen. }\end{array}$ \\
\hline $\begin{array}{l}\text { Object handling: Technology related: } \\
\text { iPad }\end{array}$ & Able to use the iPad, including apps on the device. \\
\hline $\begin{array}{l}\text { Object handling: Technology related: } \\
\text { iPhone }\end{array}$ & Able to use the iPhone \\
\hline $\begin{array}{l}\text { Object handling: Technology related: } \\
\text { Keypad use }\end{array}$ & Able to use the keypad for ATM, grocery store cashier, etc. \\
\hline $\begin{array}{l}\text { Object handling: Technology related: } \\
\text { Mouse }\end{array}$ & Able to use the computer mouse \\
\hline $\begin{array}{l}\text { Object handling: Technology related: } \\
\text { Phone }\end{array}$ & Able to use any phone \\
\hline $\begin{array}{l}\text { Object handling: Technology related: } \\
\text { Typing }\end{array}$ & Able to type on a keyboard \\
\hline $\begin{array}{l}\text { Object handling: Technology related: } \\
\text { Using a touch pad }\end{array}$ & Able to use a touch pad for a tablet computer or banking \\
\hline $\begin{array}{l}\text { Object handling: Technology related: } \\
\text { Video games }\end{array}$ & Able to play video games \\
\hline Object handling: Tray & Able to carry a tray \\
\hline $\begin{array}{l}\text { Object handling: Using a mobility } \\
\text { device }\end{array}$ & Able to use a mobility device such as a cane or walker or crutches \\
\hline $\begin{array}{l}\text { Object handling: Wallet: Items in and } \\
\text { Out }\end{array}$ & Able to transfer item $\mathrm{s}$ in and out of wallet \\
\hline Object handling: Ziploc & Able to close and open a Ziploc ${ }^{\circledR}$ bag (press $n$ seal type and sliding type) \\
\hline Object handling: Zipper & Able to open and close a zipper \\
\hline Other: Lighting a cigarette & Able to light a cigarette \\
\hline Reach: Shelf height & Able to reach shelves at various heights high medium and low. \\
\hline Reach: Take a box off a shelf & Able to get a box off a shelf \\
\hline Recreation: Biking & Able to go biking using a bicycle or tricycle \\
\hline Recreation: Gardening & Able to do gardening tasks, plant, weed, water, etc \\
\hline Recreation: General & $\begin{array}{l}\text { Able to engage in various recreational tasks like Swimming, watch TV, } \\
\text { watch sports, fishing, playing with son, reading, paddling, woodworking, } \\
\text { jewelry work, kayaking, quilting, photography, telegraph, singing, writing } \\
\text { stories, geneology research, out in the nature, walking, camping, painting, } \\
\text { watching sports, polish rocks, sewing }\end{array}$ \\
\hline Recreation: Play music & Able to play music with an instrument \\
\hline Recreation: Reading & Able to read, includes holding a book \\
\hline Recreation: Sewing & Able to sew includes threading a needle, using a sewing machine \\
\hline Recreation: Shuffling cards & Able to shuffle cards \\
\hline Recreation: Wrapping a present & Able to wrap a present \\
\hline Recreation: Yard work & Able to do yard work - mowing lawn \\
\hline Self Care: Bath & Able to bathe includes rinsing the body, applying soap, drying the body. \\
\hline Self Care: Brushing lint off clothes & Able to brush lint off clothes \\
\hline Self Care: Buttoning & Able to button clothes \\
\hline Self Care: Cleaning ears using Q-tip & Able to use Q tip to clean ears \\
\hline Self Care: Dressing & Able to dress self includes putting on dress, shirt, pants, underclothes \\
\hline $\begin{array}{l}\text { Self Care: Dressing: Shoes and } \\
\text { shoelaces }\end{array}$ & Able to don shoes and tie shoe laces \\
\hline
\end{tabular}




\section{Appendix D. Item Bank with Descriptions (Continued)}

\begin{tabular}{|l|l|}
\hline Item & Description \\
\hline Self Care: Dressing: Wearing bra & Able to wear a bra \\
\hline Self Care: Eating: Cutting meat & Able to cut the meat on the plate for eating \\
\hline Self Care: Eating: General & Able to bring spoon/ fork with food to mouth without spilling \\
\hline Self Care: Eating: Sandwich & Able to eat a sandwich \\
\hline Self Care: General & Able to take care of self \\
\hline $\begin{array}{l}\text { Self Care: Grooming: Nails - } \\
\text { finger/toe }\end{array}$ & Able to take care of nails includes cutting, applying nail polish \\
\hline $\begin{array}{l}\text { Self Care: Grooming: Putting on } \\
\text { make up/contact lens }\end{array}$ & Able to push on make up includes contact lens \\
\hline Self Care: Grooming: Shaving & Able to shave hair on face and body \\
\hline Self Care: Grooming: Wear Jewelry & Able to wear jewelry, putting on earring, fasten the back of the necklace. \\
\hline Self Care: Hair care & Able to comb and tie hair \\
\hline Self Care: Hygiene & Able to maintain general hygiene \\
\hline Self Care: Oral care: Brushing teeth & Able to brush teeth \\
\hline Self Care: Oral Care: Flossing & Able to floss teeth \\
\hline Self Care: Oral care: Using toothpick & Able to use a toothpick \\
\hline Self Care: Removing Band aid & Able to remove or apply band aid \\
\hline Self Care: Scratch back & Able to scratch back \\
\hline Self Care: Shower & Able to shower using a stand up shower \\
\hline Self Care: Sleep issues & Issues with sleep due to arm and hand problems \\
\hline $\begin{array}{l}\text { Self Care: Toileting: Bowel and } \\
\text { bladder care }\end{array}$ & Able to care for own bowel and bladder needs \\
\hline Self Care: Toileting: General & Able to maintain toilet hygiene \\
\hline $\begin{array}{l}\text { Self Care: Toileting: Using handheld } \\
\text { urinal }\end{array}$ & Able to use handheld urinal \\
\hline Self Care: Toothpaste on toothbrush & Able to apply toothpaste on toothbrush \\
\hline Self Care: Wash hands & Able to wash hands \\
\hline Self Care: Washing face & Able to wash face \\
\hline Self Care: Washing hair & Able to wash hair \\
\hline Self Care: Wring out a washcloth & Able to wring out a washcloth \\
\hline Transportation: Bus & Able to use bus for transportation \\
\hline Transportation: Driving & Able to drive \\
\hline & \\
\hline
\end{tabular}


Appendix E. Self-report Section of the Hand and Arm Function Measure Administered at Cognitive Interviews

HAND AND ARM FUNCTION QUESTIONNAIRE

Name

Age Gender Date

Diagnosis(es)

Current hand preference $\square \mathrm{R} \square \mathrm{L}$ Both

Most affected hand $\square \mathrm{R} \square \mathrm{L} \square$ Both

Least affected or unaffected hand: $\square R \square L \square$ Both

Do you have any muscle, nerve or skin issues with the hand and/or arm?

Medications taken today, dose and time

Currently residing $\square$ Hospital $\square$ Home $\square$ Nursing Home $\quad \square$ Senior Care $\square$ Other

Instructions: In this questionnaire, we want to know how difficulties with hand and arm function may have interfered with your daily activities in the past week. Write N/A (Not Applicable) if you never have the symptom.

For the questions below, we want to know about your RIGHT hand.

SYMPTOMS: RIGHT HAND

\begin{tabular}{|l|l|c|c|c|}
\hline$\#$ & $\begin{array}{l}\text { Do these symptoms interfere with } \\
\text { RIGHT hand function? }\end{array}$ & $\begin{array}{c}\text { Not at all } \\
\text { (3) }\end{array}$ & $\begin{array}{c}\text { A Little } \\
\text { (2) }\end{array}$ & $\begin{array}{c}\text { Quite a } \\
\text { lot (1) }\end{array}$ \\
\hline 1. & Coordination problems & & & \\
\hline 2. & Deformity & & & \\
\hline 3. & Fatigue & & & \\
\hline 4. & Hypersensitivity & & & \\
\hline 5. & Pain & & & \\
\hline 6. & Sensation loss & & \\
\hline 7. & Slow movements & & \\
\hline 8. & Spasticity & & & \\
\hline 9. & Stiffness & & \\
\hline 10. & Tremor & & & \\
\hline 11. & Weakness & & Subtotal & \\
\hline \multicolumn{3}{|l|}{}
\end{tabular}

(C) 2016 University of Washington - Developed by Namrata Grampurohit 
For the questions below, we want to know about your LEFT hand.

SYMPTOMS: LEFT HAND

\begin{tabular}{|c|l|c|c|c|}
\hline$\#$ & $\begin{array}{l}\text { Do these symptoms interfere with } \\
\text { LEFT hand function? }\end{array}$ & $\begin{array}{c}\text { Not at all } \\
(3)\end{array}$ & $\begin{array}{c}\text { A Little } \\
(2)\end{array}$ & $\begin{array}{c}\text { Quite a } \\
\text { lot (1) }\end{array}$ \\
\hline 12. & Coordination problems & & & \\
\hline 13. & Deformity & & & \\
\hline 14. & Fatigue & & & \\
\hline 15. & Hypersensitivity & & \\
\hline 16. & Pain & & & \\
\hline 17. & Sensation loss & & & \\
\hline 18. & Slow movements & & \\
\hline 19. & Spasticity & & & \\
\hline 20. & Stiffness & & & \\
\hline 21. & Tremor & & & \\
\hline 22. & Weakness & & Subtotal & \\
\cline { 2 - 3 } & \multicolumn{2}{|l|}{} \\
\cline { 5 - 5 }
\end{tabular}

For the questions below, think about using one or both of your hands. Write NA (Not Applicable) if you never do the task.

\section{Self care tasks}

\begin{tabular}{|c|c|c|c|c|}
\hline \# & $\begin{array}{l}\text { Do your hand function problems } \\
\text { interfere with... }\end{array}$ & $\begin{array}{l}\text { Not at all } \\
\text { (3) }\end{array}$ & $\begin{array}{l}\text { A Little } \\
\text { (2) }\end{array}$ & $\begin{array}{l}\text { Quite a } \\
\text { lot (1) }\end{array}$ \\
\hline 23. & Brushing teeth & & & \\
\hline 24. & Feeding yourself (food to mouth) & & & \\
\hline 25. & Washing hair & & & \\
\hline 26. & Getting dressed & & & \\
\hline 27. & Putting on your shoes & & & \\
\hline 28. & $\begin{array}{l}\text { Using clothing fasteners such as } \\
\text { zipper or buttons }\end{array}$ & & & \\
\hline 29. & Putting on watch or jewelry & & & \\
\hline 30. & Toilet hygiene & & & \\
\hline 31. & Cutting your finger nails & & & \\
\hline 32. & Opening a childproof pill bottle & & & \\
\hline 33. & Sleeping & & & \\
\hline
\end{tabular}


For the questions below, think about using one or both of your hands.

Write NA (Not Applicable) if you never do the task.

\section{Kitchen related tasks}

\begin{tabular}{|c|c|c|c|c|}
\hline \# & $\begin{array}{l}\text { Do your hand function problems interfere } \\
\text { with... }\end{array}$ & $\begin{array}{l}\text { Not at } \\
\text { all (3) }\end{array}$ & $\begin{array}{l}\text { A Little } \\
\text { (2) }\end{array}$ & $\begin{array}{l}\text { Quite a } \\
\text { lot (1) }\end{array}$ \\
\hline 34. & Cutting vegetables & & & \\
\hline 35. & Lifting a cup with liquid in it & & & \\
\hline 36. & Lifting a gallon of milk & & & \\
\hline 37. & Lifting a grocery bag & & & \\
\hline 38. & Opening a can & & & \\
\hline 39. & Opening a milk carton & & & \\
\hline 40. & Opening and closing a jar & & & \\
\hline 41. & Opening or closing a Ziploc $®$ bag & & & \\
\hline
\end{tabular}

\section{Miscellaneous tasks}

\begin{tabular}{|c|l|c|c|c|}
\hline$\#$ & $\begin{array}{l}\text { Do your hand function problems interfere } \\
\text { with... }\end{array}$ & $\begin{array}{c}\text { Not at } \\
\text { all (3) }\end{array}$ & $\begin{array}{c}\text { A Little } \\
(2)\end{array}$ & $\begin{array}{c}\text { Quite a } \\
\text { lot (1) }\end{array}$ \\
\hline 42. & Clapping your hands & & & \\
\hline 43. & $\begin{array}{l}\text { Getting items in and out of your wallet or } \\
\text { purse }\end{array}$ & & & \\
\hline 44. & Handling credit cards or money & & & \\
\hline 45. & Holding an open book & & & \\
\hline 46. & Shaking hands when greeting someone & & & \\
\hline 47. & Turning door knobs & & & \\
\hline 48. & Using a key to open a door lock & \multicolumn{2}{|c|}{ Subtotal } & \\
\hline \multicolumn{4}{|l|}{} \\
\hline
\end{tabular}

Technology related tasks

\begin{tabular}{|c|l|c|c|c|}
\hline$\#$ & $\begin{array}{l}\text { Do your hand function problems interfere } \\
\text { with... }\end{array}$ & $\begin{array}{c}\text { Not at } \\
\text { all (3) }\end{array}$ & $\begin{array}{c}\text { A Little } \\
(2)\end{array}$ & $\begin{array}{c}\text { Quite a } \\
\text { lot (1) }\end{array}$ \\
\hline 49. & $\begin{array}{l}\text { Pressing small buttons on a phone or } \\
\text { remote control }\end{array}$ & & & \\
\hline 50. & Using a computer keyboard & & & \\
\hline 51. & Using a computer mouse & & \\
\hline 52. & $\begin{array}{l}\text { Using a touch screen on a computer, laptop, } \\
\text { phone, or tablet }\end{array}$ & & \\
\hline \multicolumn{2}{|r|}{ Subtotal } & \\
\hline
\end{tabular}


For the questions below, think about using either or both of your hands.

Write NA (Not Applicable) if you never do the task.

(OPTIONAL) School, Recreation tasks

\begin{tabular}{|c|c|c|c|c|}
\hline \# & $\begin{array}{l}\text { Do your hand function problems interfere } \\
\text { with... }\end{array}$ & $\begin{array}{l}\text { Not at } \\
\text { all (3) }\end{array}$ & $\begin{array}{l}\text { A Little } \\
\text { (2) }\end{array}$ & $\begin{array}{l}\text { Quite a } \\
\text { lot (1) }\end{array}$ \\
\hline 53. & School in general & & & \\
\hline 54. & $\begin{array}{l}\text { Please list one task at school involving your } \\
\text { hands that is most difficult and rate it as } \\
\text { the other items }\end{array}$ & & & \\
\hline 55. & Recreational or leisure in general & & & \\
\hline 56. & $\begin{array}{l}\text { Please list one recreational or leisure task } \\
\text { involving your hands that is most difficult } \\
\text { and rate it as the other items }\end{array}$ & & & \\
\hline 57. & Childcare in general & & & \\
\hline 58. & $\begin{array}{l}\text { Please list one childcare task involving your } \\
\text { hands that is most difficult and rate it as } \\
\text { the other items }\end{array}$ & & & \\
\hline 59. & Work in general (paid or volunteer work) & & & \\
\hline 60. & $\begin{array}{l}\text { Please list one task at work involving your } \\
\text { hands that is most difficult and rate it as } \\
\text { the other items }\end{array}$ & & & \\
\hline
\end{tabular}

Thank you for completing this questionnaire! 


\section{Scoring for Self-report Section of Hand and Arm Function Measure}

\begin{tabular}{|l|l|}
\hline Tasks & Score \\
\hline Right hand symptom subtotal (out of 33) & \\
\hline Left hand symptom subtotal (out of 33) & \\
\hline Self Care Subtotal (out of 33) & \\
\hline Kitchen Subtotal (out of 24) & \\
\hline Miscellaneous Subtotal (out of 21) & \\
\hline Technology Subtotal (out of 12) & \\
\hline (Optional) Childcare, school, work and recreational subtotal (out of 24) & \\
\hline Total Raw Score (out of 180) & \\
\hline
\end{tabular}

In order to compute percent score:

- Use Method I, if none of the items were missed (or marked NA) by the patient.

- Use Method II, if there are missed (or marked NA) responses.

METHOD I

\begin{tabular}{|l|l|}
\hline Final Percent Score $=\frac{\text { TotalRawScore }}{\text { MaxPointsPossible }} \times 100$ & \\
\hline
\end{tabular}

Final Percent Score is out of 100 (higher is better)

METHOD II (Use only if there were missed or NA items in responses)

\begin{tabular}{|c|c|l|}
\hline Step 1 & Number of missed tasks & \\
\hline Step 2 & MissedPoints $=$ MissedTasks x 3 & \\
\hline Step 3 & MaxPossiblePoints $=180-$ MissedPoints & \\
\hline Step 4 & Final Percent Score $=\frac{\text { TotalRawScore }}{\text { MaxPointsPossible }} \times 100$ & \\
\hline
\end{tabular}

Final Percent Score is out of 100 (higher is better)

Final Percent score is the self reported hand function ability for an individual to engage in daily activities. 
Appendix F. Performance-based Section of the Hand and Arm Function Measure Administered at Cognitive Interviews

\section{ARM AND HAND FUNCTION PERFORMANCE MEASURE INSTRUCTIONS}

\section{Materials}

- 15 American pennies

- 15 Dry black eye peas

- Bowl (18 oz. Glass/ ceramic cereal bowl 6"diameter at the rim)

- Can of beans with contents sealed (1 lb. $16 \mathrm{oz}$. e.g., Bush's red kidney beans)

- Coffee mug empty (12 oz., ceramic or glass)

- Empty plastic spice bottle (height 4" with screw top lid)

- Masking tape

- Metal teaspoon

- Padlock and key $\left(11 / 8^{\text {th }}\right.$ inch wide)

- Pencil (sharpened Standard 2HB at least 6" long Eraser optional)

- Plastic jar with screw top lid (sealed, diameter 2.7"; height 4.7" e.g., Jiff peanut butter jar)

- Stopwatch

- Tape Measure

- Writing paper (writing pad $81 / 2 " \times 113 / 4$ " white ruled $1 / 4$ " between horizontal lines)

\section{Sensory testing kit}

- American penny

- Cotton ball

- Kidney bean (large, dry, red)

- Nut and bolt (nut hexagonal diameter $1 / 2$ "; bolt diameter $3 / 8$ ")

- One pair adult tube socks (opaque, thick, large, 12" long, unused; e.g., American hospital socks)

- Paper clip (28 mm)

- Standard metal key (1.6" long)

Writing sample address

- Type the address below in font size 18 (large)

- Arial typeface (sans serif), lamination preferred.

Jane Doe

4567 NE Brown St. Greenwood, WA 98213

\section{Positioning}

The patient or client is referred to as the 'individual' in this test. The individual is seated at a table with feet flat on the floor for assessment (Fig. 1). If seated in a wheelchair, the wheelchair should be locked and armrests removed, provided person has good trunk control. The individual should be seated close to the table so the forearms can rest on the table. Any deviations to this seating must be noted. For consistency in testing, repeated assessments should be conducted in the same seating position. If similar seating is not possible, consistent height of the seat and table should be maintained.

\section{Reach distance and midline marking}

Once the individual is seated at the testing table, you will need to determine reach distance and midline. To determine the reach distance, while the individual is seated upright, ask him/her to actively reach forward

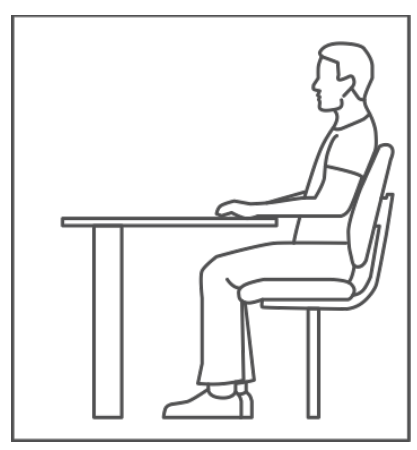

Fig. 1: Seating position with both hands with the elbows fully extended, open hand, forearm pronated and flat on the table. Individual's back should stay in contact with the seatback during this measurement. The point where the wrist is located is considered the reach distance.

(C) 2016 University of Washington - Developed by Namrata Grampurohit 
Mark the reach distance with a piece of masking tape on the table placed horizontally parallel to the front edge of the table. At the individual's midline, place masking tape extending from the reach distance marker to the front edge of the table. Measure and note the reach distance (Fig. 2). Use this distance for any repeated testing sessions. This is essential for evidence for reliability of scores. Place a 6 -inch marker on either side of this midline marker.

\section{Accommodations}

If individual is unable achieve full elbow extension, the therapist can mark the reach distance of the wrist at their maximal active elbow extension and write a note indicating this modification. If an individual has severe limitations with one or both arms, use the less involved side for determining the reach distance.

\section{Administration}

Prior to testing, all the materials should be cleaned and ready to use. The instructions given under each task must be read aloud to the individual. Interpreters should be used for non-English speakers. Individuals being tested should be informed to wear their

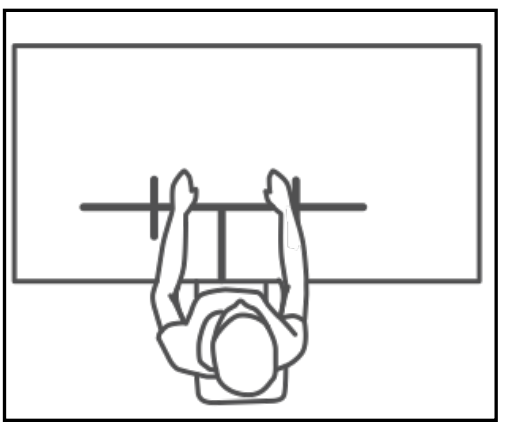

Fig 2: Reach distance, midline and 6 inch marking glasses during the assessment session if they routinely use them.

The therapist should ensure that the person's sleeves are not restricting reach. For hygienic concerns, dry beans (black eyed peas and kidney beans for sensory test) should only be used one time and then discarded. If items such as bean cans and peanut butter jar break or leak, they should be replaced. For timed items, if objects are dropped on the floor, the individual must continue with the task while the therapist replaces the dropped item (e.g., putting a coin back in the bowl). However, if during a timed task a dropped item (e.g., bowl) causes the individual to completely stop the task, the task may be readministered up to 3 times.

\section{Demonstration}

If therapist determines that individual is having difficulty understanding the item, tasks may be demonstrated.

\section{Scoring Description}

\begin{tabular}{l|l|l} 
Score & Description & Details \\
\hline 5 & $\begin{array}{l}\text { Task completed } \\
\text { independently }\end{array}$ & $\begin{array}{l}\text { Task is completed within a reasonable time frame and with good } \\
\text { quality of movement }\end{array}$ \\
\hline 4 & $\begin{array}{l}\text { Task completed } \\
\text { independently with } \\
\text { compensation }\end{array}$ & $\begin{array}{l}\text { Compensation }-1 \text { ) increased time 2) modified technique (e.g. } \\
\text { using less affected/ intact side to assist, shoulder shrugs, trunk } \\
\text { leaning) 3) adaptive devices or orthotics }\end{array}$ \\
\hline 3 & $\begin{array}{l}\text { Task completed with } \\
\text { difficulty }\end{array}$ & $\begin{array}{l}\text { Difficulty - Observed impairment related to 1) poor quality of } \\
\text { movement 2) pain 3) extensive time 4) task compensations that } \\
\text { compromise safety (i.e. dropped items) }\end{array}$ \\
\hline 2 & Task partially completed & Independently completes $\geq 50 \%$ of task. \\
\hline 1 & Task Attempted & Task attempted, less than $50 \%$ of task completed independently \\
\hline 0 & Not able to do & Task not attempted, unable to carry out any part of the task
\end{tabular}




\section{HAND AND ARM FUNCTION PERFORMANCE MEASURE}

Name

Age Gender

Date

Diagnosis(es)

Therapist Name

Assessment $\square$ Initial $\square$ Follow up $\square$ Discharge

Most affected hand $\square \mathbf{R} \square \mathbf{L} \square$ Both

Least or unaffected hand $\square \mathbf{R} \square \mathbf{L} \square$ Both

Current Dominance $\square \mathbf{R} \square \mathbf{L} \square$ Both

Previous Dominance: $\square$ R $\square$ L $\square$ Both $\square$ Same

Any muscle, nerve or skin issues with the hand and/or

arm

Medications taken today, dose and time

Pain in arm or hand

Right (no pain) 012345678910 (severe)

Left (no pain) 012345678910 (severe)

Pain location: Right Left

Adaptive devices or orthotics used during the test 


\begin{tabular}{|c|c|c|}
\hline $\begin{array}{l}\text { Therapist Instructions } \\
\text { Drepare all materials before } \\
\text { starting the test. } \\
\text { Administer each item and mark } \\
\text { he task score. Give the } \\
\text { ndividual as much time as } \\
\text { needed to complete each task. } \\
\text { As you administer the tasks, } \\
\text { lease write down any difficulties } \\
\text { observed and any strategies the } \\
\text { ndividual uses during the task. } \\
\text { =or example, compensation } \\
\text { could be using both hands for } \\
\text { unimanual tasks, leaning the } \\
\text { runk, bracing against the table, } \\
\text { adaptive devices, compensations } \\
\text { or vision issues such as moving } \\
\text { objects closer or to the side, etc. } \\
\text { Directions to be read to the } \\
\text { ndividual are in italics. } \\
\text { nformation for the administrator } \\
\text { s in regular type. The therapist } \\
\text { nay demonstrate the task to the } \\
\text { ndividual along with the verbal } \\
\text { nstructions. The therapist may } \\
\text { change the order of hands for } \\
\text { unimanual tasks (left and then } \\
\text { ight) if desired. }\end{array}$ & $\begin{array}{l}\text { Seating surface (e.g., chair): } \\
\text { Table specifications (e.g., height): } \\
\text { Reach distance (forward): } \\
\text { Individual position: Individual } \\
\text { seated at the table with elbows on } \\
\text { the table. Please keep the seating } \\
\text { and table arrangement consistent } \\
\text { for repeated testing. }\end{array}$ & $\begin{array}{l}\text { Individual Instructions } \\
\text { "For this test, you are asked to } \\
\text { move your arm and hand, and } \\
\text { reach, grasp and place some } \\
\text { objects. This will help us learn } \\
\text { how you use your hands for } \\
\text { daily activities. Some tasks at } \\
\text { the end of the test are timed } \\
\text { and I will tell you when the } \\
\text { timed tasks begin. I will show } \\
\text { you how to do each task as we } \\
\text { go along if needed. There are } \\
10 \text { tasks that I want you to do." } \\
\text { "Do you have any questions? } \\
\text { Are you ready to begin?" }\end{array}$ \\
\hline
\end{tabular}




\begin{tabular}{|c|c|c|c|c|c|c|c|}
\hline \multirow[t]{2}{*}{$\begin{array}{l}\text { Unimanual Tasks } \\
\text { Instructions: "In the first set of } \\
\text { tasks, you will have to move } \\
\text { the hand and arm on each side } \\
\text { separately, right and then left." } \\
\text { 1. Lower back }\end{array}$} & SIDE & $\begin{array}{l}\text { Indepen- } \\
\text { dently } \\
\text { (5) }\end{array}$ & $\begin{array}{l}\begin{array}{c}\text { With } \\
\text { compen- } \\
\text { sation }\end{array} \\
\text { (4) }\end{array}$ & $\begin{array}{c}\text { With } \\
\text { difficulty } \\
\text { (3) }\end{array}$ & $\begin{array}{l}\text { Partially } \\
\text { completed } \\
\text { (2) }\end{array}$ & $\begin{array}{l}\text { Attempted } \\
\text { (1) }\end{array}$ & $\begin{array}{l}\text { Not } \\
\text { able to } \\
\text { do } \\
(0)\end{array}$ \\
\hline & Right & & & & & & \\
\hline $\begin{array}{l}\text { "Take your right hand behind } \\
\text { your body to touch your } \\
\text { lower back with the palm of } \\
\text { your hand." "Now use your } \\
\text { left hand." } \\
\text { Therapist Notes: }\end{array}$ & Left & & & & & & \\
\hline \multirow[t]{2}{*}{$\begin{array}{l}\text { 2. Coffee mug } \\
\text { Set up: Therapist places an } \\
\text { empty coffee mug at midline on } \\
\text { the table at reach distance. } \\
\text { "Pick up the coffee cup with } \\
\text { your right hand, pretend to } \\
\text { drink from it and place it back } \\
\text { on the table." }\end{array}$} & Right & & & & & & \\
\hline & Left & & & & & & \\
\hline \multirow[t]{2}{*}{$\begin{array}{l}\text { 3. Bean can RIGHT HAND } \\
\text { Place unopened bean can on } \\
\text { the 6inch marker on the RIGHT } \\
\text { side. "Using your right hand, } \\
\text { move the bean can back and } \\
\text { forth } 10 \text { times. Be sure to stay } \\
\text { on the } 6 \text { inch marker on either } \\
\text { side and not drag the can." } \\
\text { Therapist Notes: }\end{array}$} & $\begin{array}{l}\text { Move } \\
\text { LEFT }\end{array}$ & & & & & & \\
\hline & $\begin{array}{c}\text { Move } \\
\text { RIGHT }\end{array}$ & & & & & & \\
\hline
\end{tabular}




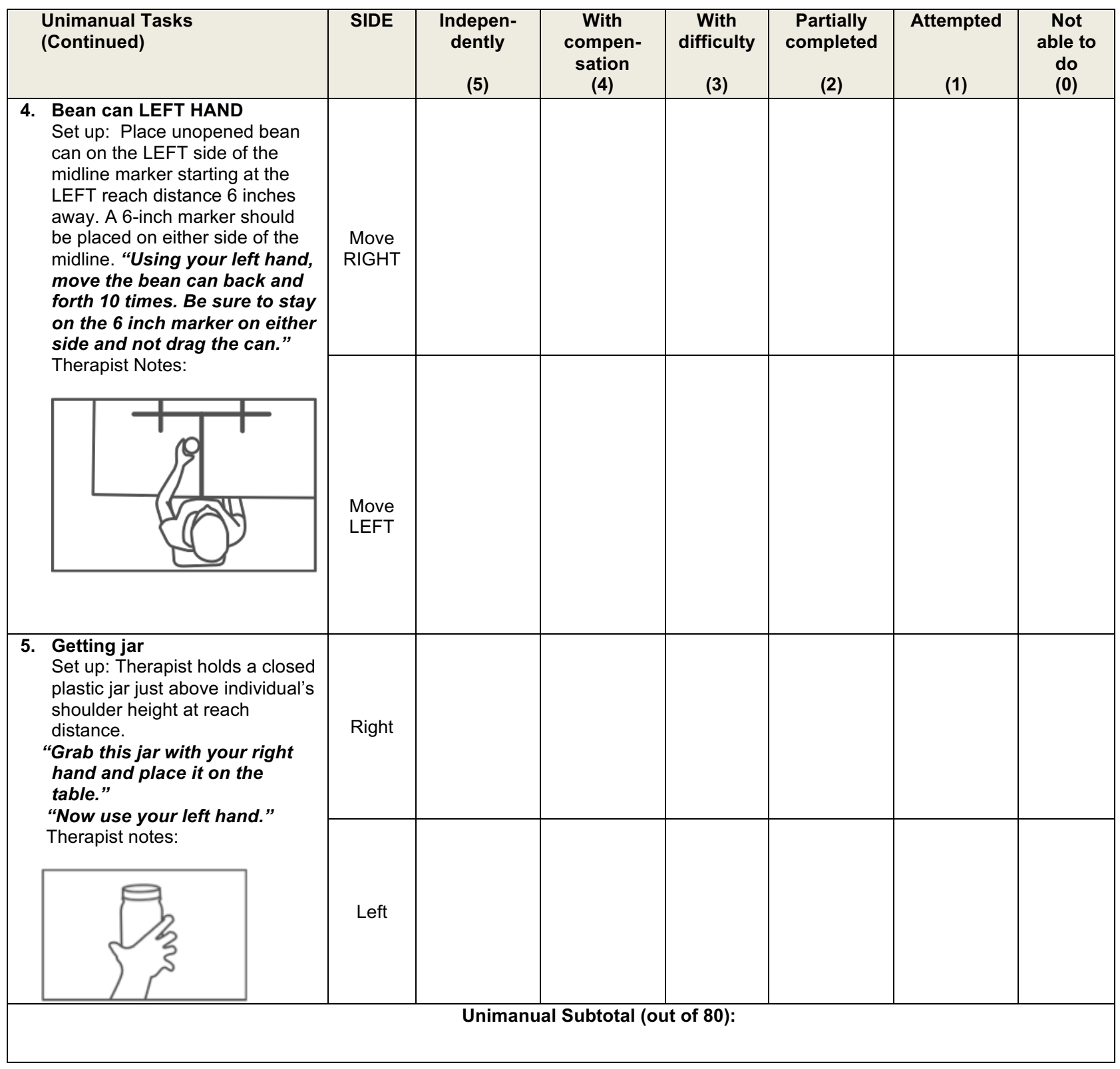




\begin{tabular}{|c|c|c|c|c|c|c|c|}
\hline $\begin{array}{l}\text { Bimanual Tasks } \\
\text { Instructions: "In the next set } \\
\text { of tasks you need to use both } \\
\text { hands together." }\end{array}$ & SIDE & $\begin{array}{l}\text { Indepen- } \\
\text { dently } \\
\text { (5) }\end{array}$ & $\begin{array}{l}\text { With } \\
\text { compen- } \\
\text { sation } \\
(4)\end{array}$ & $\begin{array}{c}\text { With } \\
\text { difficulty } \\
\text { (3) }\end{array}$ & $\begin{array}{c}\text { Partially } \\
\text { completed } \\
\text { (2) }\end{array}$ & $\begin{array}{l}\text { Attempted } \\
\text { (1) }\end{array}$ & $\begin{array}{l}\text { Not able to } \\
\text { do } \\
\text { (0) }\end{array}$ \\
\hline $\begin{array}{l}\text { Opening jar } \\
\text { Set up: Place an unopened } \\
\text { screw top plastic jar on the } \\
\text { table at midline reach } \\
\text { distance away. } \\
\text { "Open this jar using both } \\
\text { of your hands. Place the lid } \\
\text { on the table when you are } \\
\text { done." } \\
\text { Therapist Notes: }\end{array}$ & $\begin{array}{l}\text { Hand } \\
\text { on lid } \\
\square \mathbf{R} \square \mathbf{L} \\
\text { Mark } \\
\text { one }\end{array}$ & & & & & & \\
\hline $\begin{array}{l}7 . \\
\text { Open lock }\end{array}$ & $\begin{array}{l}\text { Key in } \\
\text { hand } \\
\square \mathbf{R} \square \mathbf{L} \\
\text { Mark } \\
\text { one }\end{array}$ & & & & & & \\
\hline $\begin{array}{l}\text { Open spice bottle } \\
\text { Set up: } \\
\text { Place an } \\
\text { empty } \\
\text { spice } \\
\text { bottle } \\
\text { (twist type } \\
\text { in closed } \\
\text { position) }\end{array}$ & $\begin{array}{l}\text { Hand } \\
\text { on lid } \\
\square \mathbf{R} \square \mathbf{L} \\
\text { Mark } \\
\text { one }\end{array}$ & & & & & & \\
\hline \multicolumn{8}{|c|}{10} \\
\hline
\end{tabular}




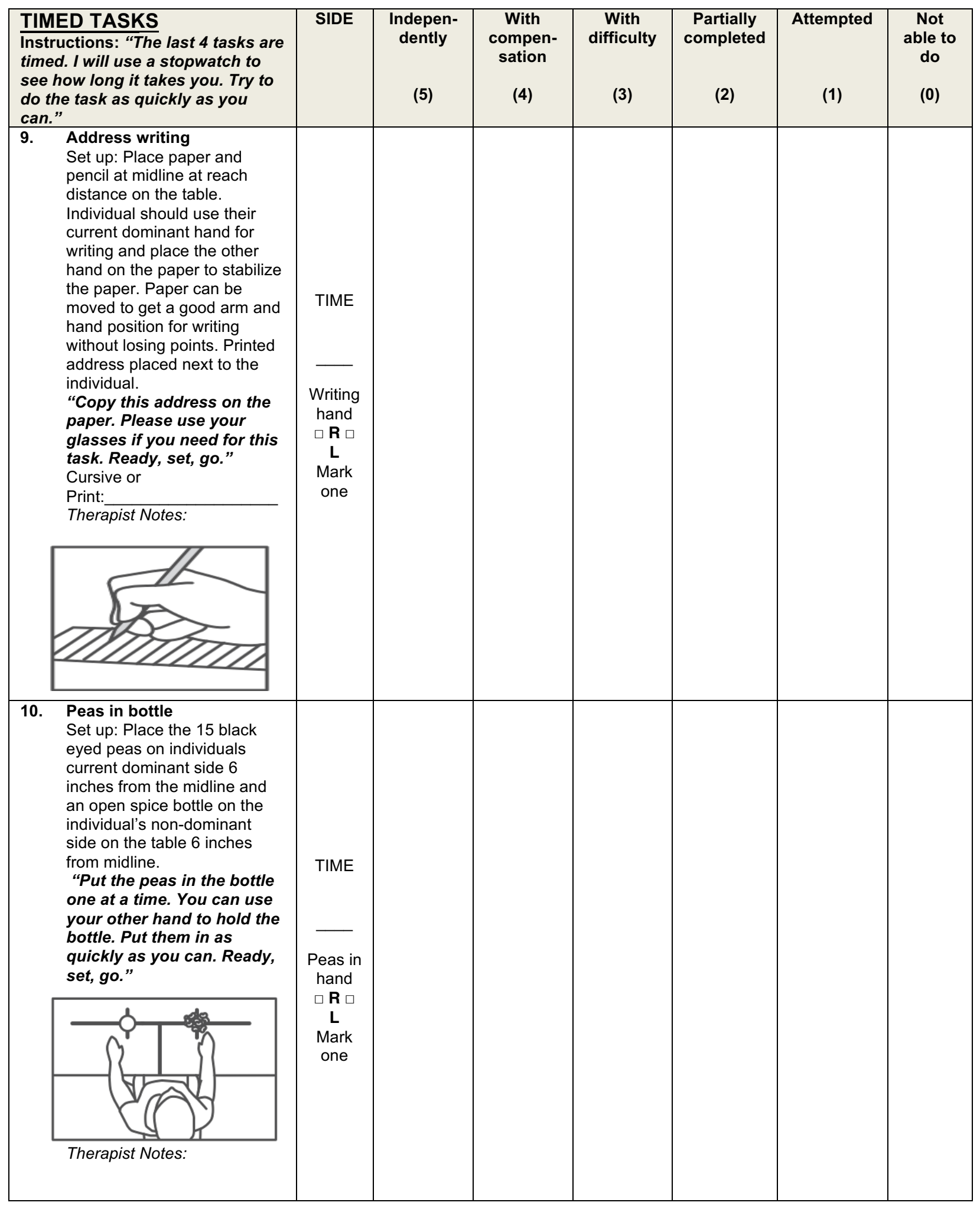




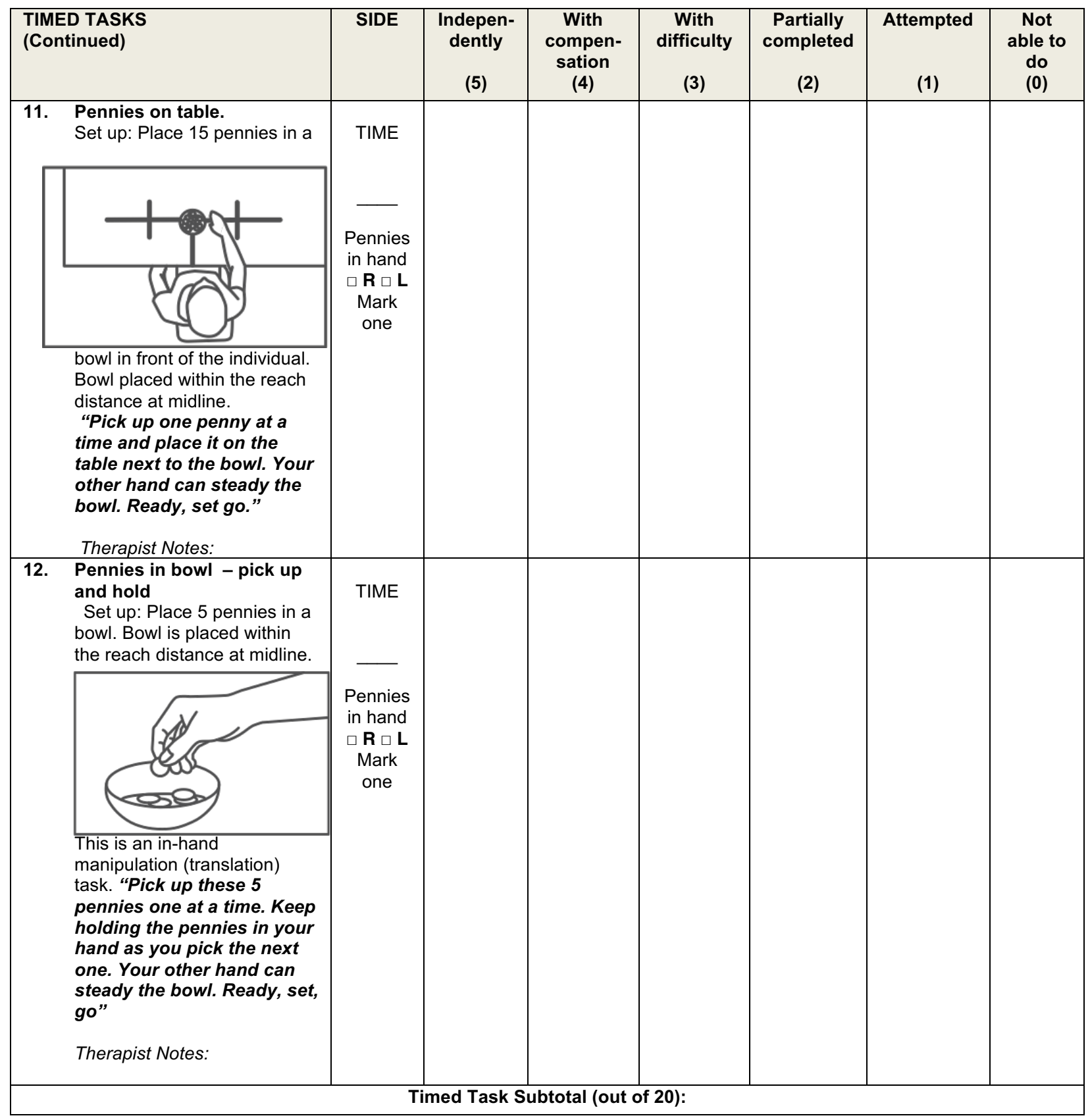

Scoring

Add the Unimanual, Bimanual and Timed subtotals to get the Total Raw Score.

\begin{tabular}{|l|l|l|}
\hline Unimanual subtotal (out of 80) & \multirow{2}{*}{ Total Raw Score (out of 115) } \\
\hline Bimanual subtotal (out of 15) & & \\
\hline Timed task subtotal (out of 20) & & \\
\hline
\end{tabular}

To convert Raw Score into Percent Score

\begin{tabular}{|c|l|}
\hline Final Percent Score $=\frac{\text { TotalRawScore }}{115} \times 100$ & Percent Score (out of 100) \\
\hline
\end{tabular}

Final Percent Score is out of 100 (higher is better) and it is the hand function ability for an individual to engage in daily tasks involving reach, grasp, manipulation, and dexterity. 


\section{REFERENCES}

Abdullah, H. A., Tarry, C., Lambert, C., Barreca, S., \& Allen, B. O. (2011). Results of clinicians using a therapeutic robotic system in an inpatient stroke rehabilitation unit. Journal of Neuroengineering and Rehabilitation, 8, 50. http://doi.org/10.1186/1743-0003-8-50

Adamovich, S. V., Fluet, G. G., Mathai, A., Qiu, Q., Lewis, J., \& Merians, A. S. (2009). Design of a complex virtual reality simulation to train finger motion for persons with hemiparesis: a proof of concept study. Journal of Neuroengineering and Rehabilitation, 6, 28. http://doi.org/10.1186/1743-0003-6-28

Ahmed, S., Mayo, N. E., Higgins, J., Salbach, N. M., Finch, L., \& Wood-Dauphinée, S. L. (2003). The Stroke Rehabilitation Assessment of Movement (STREAM): a comparison with other measures used to evaluate effects of stroke and rehabilitation. Physical Therapy, 83(7), 617-630.

Alberta, F. G., ElAttrache, N. S., Bissell, S., Mohr, K., Browdy, J., Yocum, L., \& Jobe, F. (2010). The development and validation of a functional assessment tool for the upper extremity in the overhead athlete. The American Journal of Sports Medicine, 38(5), 903911. http://doi.org/10.1177/0363546509355642

Allen, M. J., \& Yen, W. M. (2002). Introduction to measurement theory. Prospect Heights, Ill.: Waveland Press.

Al Snih, S., Markides, K. S., Ottenbacher, K. J., \& Raji, M. A. (2004). Hand grip strength and incident ADL disability in elderly Mexican Americans over a seven-year period. Aging Clinical and Experimental Research, 16(6), 481-486. 
Alt Murphy, M., Resteghini, C., Feys, P., \& Lamers, I. (2015). An overview of systematic reviews on upper extremity outcome measures after stroke. BMC Neurology, 15, 29. http://doi.org/10.1186/s12883-015-0292-6

Alt Murphy, M., Willén, C., \& Sunnerhagen, K. S. (2012). Movement kinematics during a drinking task are associated with the activity capacity level after stroke. Neurorehabilitation and Neural Repair, 26(9), 1106-1115. http://doi.org/10.1177/1545968312448234

American Educational Research Association. (1999). Validity: Part 1, Standards of Educational testing.

American Occupational Therapy Association. (2010). Workforce trends in Occupational therapy. American Occupational Therapy Association. Retrieved from https://www.aota.org//media/Corporate/Files/EducationCareers/Prospective/Workforce-trends-in-OT.PDF

Anderson, C. (2010). Presenting and Evaluating Qualitative Research. American Journal of Pharmaceutical Education, 74(8), 1-7.

Angst, F., Goldhahn, J., Drerup, S., Kolling, C., Aeschlimann, A., Simmen, B. R., \& Schwyzer, H. K. (2012). Responsiveness of five outcome measurement instruments in total elbow arthroplasty. Arthritis Care \& Research, 64(11), 1749-1755. http://doi.org/10.1002/acr.21744

Annear, M. J., Toye, C. M., Eccleston, C. E., McInerney, F. J., Elliott, K. E. J., Tranter, B. K., ... Robinson, A. L. (2015). Dementia Knowledge Assessment Scale: Development and Preliminary Psychometric Properties. Journal of the American Geriatrics Society, 63(11), 2375-2381. http://doi.org/10.1111/jgs.13707 
Arya, K. N., \& Pandian, S. (2013). Effect of task-based mirror therapy on motor recovery of the upper extremity in chronic stroke patients: a pilot study. Topics in Stroke Rehabilitation, 20(3), 210-217. http://doi.org/10.1310/tsr2003-210

Asher, I. (2007). Occupational Therapy Assessment Tools: An Annotated Index, 3rd Edition (3rd ed.). AOTA Press.

Ashford, S., Slade, M., Malaprade, F., \& Turner-Stokes, L. (2008). Evaluation of functional outcome measures for the hemiparetic upper limb: a systematic review. Journal of Rehabilitation Medicine: Official Journal of the UEMS European Board of Physical and Rehabilitation Medicine, 40(10), 787-795. http://doi.org/10.2340/16501977-0276

Ashford, S., \& Turner-Stokes, L. (2013). Systematic review of upper-limb function measurement methods in botulinum toxin intervention for focal spasticity. Physiotherapy Research International: The Journal for Researchers and Clinicians in Physical Therapy, 18(3), 178-189. http://doi.org/10.1002/pri.1554

Ashford, S., Turner-Stokes, L., Siegert, R., \& Slade, M. (2013). Initial psychometric evaluation of the Arm Activity Measure (ArmA): a measure of activity in the hemiparetic arm. Clinical Rehabilitation, 27(8), 728-740. http://doi.org/10.1177/0269215512474942

Au-Yeung, S. S. Y., \& Hui-Chan, C. W. Y. (2014). Electrical acupoint stimulation of the affected arm in acute stroke: a placebo-controlled randomized clinical trial. Clinical Rehabilitation, 28(2), 149-158. http://doi.org/10.1177/0269215513494875

Baker, K., Barrett, L., Playford, E. D., Aspden, T., Riazi, A., \& Hobart, J. (2015). Measuring arm function early after stroke: is the DASH good enough? Journal of Neurology, Neurosurgery, and Psychiatry. http://doi.org/10.1136/jnnp-2015-310557 
Barreca, S., Gowland, C., Stratford, P., Huijbregts, M., Griffiths, J., Torresin, W., .. Masters, L. (2004). Development of the Chedoke Arm and Hand Activity Inventory: theoretical constructs, item generation, and selection. Topics in Stroke Rehabilitation, 11(4), 31-42. http://doi.org/10.1310/JU8P-UVK6-68VW-CF3W

Barreca, S. R., Stratford, P. W., Lambert, C. L., Masters, L. M., \& Streiner, D. L. (2005). Testretest reliability, validity, and sensitivity of the Chedoke arm and hand activity inventory: a new measure of upper-limb function for survivors of stroke. Archives of Physical Medicine and Rehabilitation, 86(8), 1616-1622. http://doi.org/10.1016/j.apmr.2005.03.017

Barreca, S. R., Stratford, P. W., Masters, L. M., Lambert, C. L., Griffiths, J., \& McBay, C. (2006). Validation of Three Shortened Versions of the Chedoke Arm and Hand Activity Inventory. Physiotherapy Canada, 58(2), 148-156. http://doi.org/10.3138/ptc.58.2.148

Bartolo, M., De Nunzio, A. M., Sebastiano, F., Spicciato, F., Tortola, P., Nilsson, J., \& Pierelli, F. (2014). Arm weight support training improves functional motor outcome and movement smoothness after stroke. Functional Neurology, 29(1), 15-21.

Beebe, J. A., \& Lang, C. E. (2009). Relationships and responsiveness of six upper extremity function tests during the first six months of recovery after stroke. Journal of Neurologic Physical Therapy: JNPT, 33(2), 96-103. http://doi.org/10.1097/NPT.0b013e3181a33638

Berglund, K., \& Fugl-Meyer, A. R. (1986). Upper extremity function in hemiplegia. A crossvalidation study of two assessment methods. Scandinavian Journal of Rehabilitation Medicine, 18(4), 155-157. 
Bernspång, B., Asplund, K., Eriksson, S., \& Fugl-Meyer, A. R. (1987). Motor and perceptual impairments in acute stroke patients: effects on self-care ability. Stroke; a Journal of Cerebral Circulation, 18(6), 1081-1086.

Blennerhassett, J. M., Avery, R. M., \& Carey, L. M. (2010). The test-retest reliability and responsiveness to change for the Hand Function Survey during stroke rehabilitation. Australian Occupational Therapy Journal, 57(6), 431-438. http://doi.org/10.1111/j.14401630.2010.00884.x

Bohannon, R. (1999). Motricity Index scores are valid indicator of paretic upper extremity strength following stroke. Journal of Physical Therapy Science, 11, 59-61.

Borstad, A. L., Bird, T., Choi, S., Goodman, L., Schmalbrock, P., \& Nichols-Larsen, D. S. (2013). Sensorimotor training and neural reorganization after stroke: a case series. Journal of Neurologic Physical Therapy: JNPT, 37(1), 27-36. http://doi.org/10.1097/NPT.0b013e318283de0d

Bowman, M. H., Taub, E., Uswatte, G., Delgado, A., Bryson, C., Morris, D. M., ... Mark, V. W. (2006). A treatment for a chronic stroke patient with a plegic hand combining CI therapy with conventional rehabilitation procedures: case report. NeuroRehabilitation, 21(2), $167-176$.

Boyne, P., Dunning, K., Levine, P., Hermann, V. H., \& Page, S. J. (2010). Orthotic functional electrical stimulation following botulinum toxin for a young adult with severe hand impairment due to childhood stroke. Physiotherapy Theory and Practice, 26(4), 267-274. http://doi.org/10.3109/09593980902967188

Brauer, S. G., Hayward, K. S., Carson, R. G., Cresswell, A. G., \& Barker, R. N. (2013). The efficacy of SMART Arm training early after stroke for stroke survivors with severe upper 
limb disability: a protocol for a randomised controlled trial. BMC Neurology, 13, 71. http://doi.org/10.1186/1471-2377-13-71

Broderick, J. E., Schneider, S., Junghaenel, D. U., Schwartz, J. E., \& Stone, A. A. (2013). Validity and reliability of patient-reported outcomes measurement information system instruments in osteoarthritis. Arthritis Care \& Research, 65(10), 1625-1633. http://doi.org/10.1002/acr.22025

Broeks, J. G., Lankhorst, G. J., Rumping, K., \& Prevo, A. J. (1999). The long-term outcome of arm function after stroke: results of a follow-up study. Disability and Rehabilitation, 21(8), 357-364.

Brogårdh, C., \& Lexell, J. (2010). A 1-year follow-up after shortened constraint-induced movement therapy with and without mitt poststroke. Archives of Physical Medicine and Rehabilitation, 91(3), 460-464. http://doi.org/10.1016/j.apmr.2009.11.009

Brogårdh, C., \& Sjölund, B. H. (2006). Constraint-induced movement therapy in patients with stroke: a pilot study on effects of small group training and of extended mitt use. Clinical Rehabilitation, 20(3), 218-227.

Brunner, I. C., Skouen, J. S., \& Strand, L. I. (2012). Is modified constraint-induced movement therapy more effective than bimanual training in improving arm motor function in the subacute phase post stroke? A randomized controlled trial. Clinical Rehabilitation, 26(12), 1078-1086. http://doi.org/10.1177/0269215512443138

Brunnstrom, S. (1966). Motor testing procedures in hemiplegia: based on sequential recovery stages. Physical Therapy, 46(4), 357-75. 
Buneo, C. A., Jarvis, M. R., Batista, A. P., \& Andersen, R. A. (2002). Direct visuomotor transformations for reaching. Nature, 416(6881), 632-636. http://doi.org/10.1038/416632a

Burns, A., Burridge, J., \& Pickering, R. (2007). Does the use of a constraint mitten to encourage use of the hemiplegic upper limb improve arm function in adults with subacute stroke? Clinical Rehabilitation, 21(10), 895-904. http://doi.org/10.1177/0269215507079144

Canny, M. L., Thompson, J. M., \& Wheeler, M. J. (2009). Reliability of the box and block test of manual dexterity for use with patients with fibromyalgia. The American Journal of Occupational Therapy: Official Publication of the American Occupational Therapy Association, 63(4), 506-510.

Cano, S. J., Barrett, L. E., Zajicek, J. P., \& Hobart, J. C. (2011). Beyond the reach of traditional analyses: using Rasch to evaluate the DASH in people with multiple sclerosis. Multiple Sclerosis (Houndmills, Basingstoke, England), 17(2), 214-222. http://doi.org/10.1177/1352458510385269

Carlozzi, N. E., Tulsky, D. S., \& Kisala, P. A. (2011). Traumatic brain injury patient-reported outcome measure: identification of health-related quality-of-life issues relevant to individuals with traumatic brain injury. Archives of Physical Medicine and Rehabilitation, 92(10 Suppl), S52-60. http://doi.org/10.1016/j.apmr.2010.12.046

Carmeli, E., Patish, H., \& Coleman, R. (2003). The aging hand. The Journals of Gerontology. Series A, Biological Sciences and Medical Sciences, 58(2), 146-152.

Carod-Artal, F. J., Ferreira Coral, L., Stieven Trizotto, D., \& Menezes Moreira, C. (2009). Selfand proxy-report agreement on the Stroke Impact Scale. Stroke; a Journal of Cerebral Circulation, 40(10), 3308-3314. http://doi.org/10.1161/STROKEAHA.109.558031 
Carr, J. H., Shepherd, R. B., Nordholm, L., \& Lynne, D. (1985). Investigation of a new motor assessment scale for stroke patients. Physical Therapy, 65(2), 175-180.

Carroll, D. (1965). A Quantitative test of upper extremity function. Journal of Chronic Diseases, $18,479-491$.

Celik, O., O’Malley, M. K., Boake, C., Levin, H. S., Yozbatiran, N., \& Reistetter, T. A. (2010). Normalized movement quality measures for therapeutic robots strongly correlate with clinical motor impairment measures. IEEE Transactions on Neural Systems and Rehabilitation Engineering: A Publication of the IEEE Engineering in Medicine and Biology Society, 18(4), 433-444. http://doi.org/10.1109/TNSRE.2010.2047600

Cella, D. (2015). Neuro-QoL. Retrieved October 22, 2015, from http://www.neuroqol.org/Pages/default.aspx

Cella, D., Lai, J. S., Nowinski, C. J., Victorson, D., Peterman, A., Miller, D., ... Moy, C. (2012). Neuro-QOL: brief measures of health-related quality of life for clinical research in neurology. Neurology, 78(23), 1860-1867. http://doi.org/10.1212/WNL.0b013e318258f744

Chae, J., Harley, M. Y., Hisel, T. Z., Corrigan, C. M., Demchak, J. A., Wong, Y. T., \& Fang, Z. P. (2009). Intramuscular electrical stimulation for upper limb recovery in chronic hemiparesis: an exploratory randomized clinical trial. Neurorehabilitation and Neural Repair, 23(6), 569-578. http://doi.org/10.1177/1545968308328729

Chae, J., Labatia, I., \& Yang, G. (2003). Upper limb motor function in hemiparesis: concurrent validity of the Arm Motor Ability test. American Journal of Physical Medicine \& Rehabilitation / Association of Academic Physiatrists, 82(1), 1-8. http://doi.org/10.1097/01.PHM.0000034950.95737.91 
Chan, M. K. L., Tong, R. K. Y., \& Chung, K. Y. K. (2009). Bilateral upper limb training with functional electric stimulation in patients with chronic stroke. Neurorehabilitation and Neural Repair, 23(4), 357-365. http://doi.org/10.1177/1545968308326428

Chen, \& Bode, R. K. (2010). Psychometric validation of the Manual Ability Measure-36 (MAM36) in patients with neurologic and musculoskeletal disorders. Archives of Physical Medicine and Rehabilitation, 91(3), 414-420. http://doi.org/10.1016/j.apmr.2009.11.012

Chen, Granger, C. V., Peimer, C. A., Moy, O. J., \& Wald, S. (2005). Manual Ability Measure (MAM-16): a preliminary report on a new patient-centred and task-oriented outcome measure of hand function. Journal of Hand Surgery (Edinburgh, Scotland), 30(2), 207216. http://doi.org/10.1016/j.jhsb.2004.12.005

Chen, H., Lin, K., Wu, C., \& Chen, C. (2012). Rasch validation and predictive validity of the action research arm test in patients receiving stroke rehabilitation. Archives of Physical Medicine and Rehabilitation, 93(6), 1039-1045. http://doi.org/10.1016/j.apmr.2011.11.033

Chen, H.M., Chen, C. C., Hsueh, I.P., Huang, S.L., \& Hsieh, C.L. (2009). Test-retest reproducibility and smallest real difference of 5 hand function tests in patients with stroke. Neurorehabilitation and Neural Repair, 23(5), 435-440. http://doi.org/10.1177/1545968308331146

Chen, H.M., Hsieh, C.L., Sing Kai Lo, Liaw, L.J., Chen, S.M., \& Lin, J.H. (2007). The test-retest reliability of 2 mobility performance tests in patients with chronic stroke. Neurorehabilitation and Neural Repair, 21(4), 347-352. http://doi.org/10.1177/1545968306297864 
Chen, S., Lewthwaite, R., Schweighofer, N., \& Winstein, C. J. (2013). Discriminant validity of a new measure of self-efficacy for reaching movements after stroke-induced hemiparesis. Journal of Hand Therapy: Official Journal of the American Society of Hand Therapists, 26(2), 116-122; quiz 123. http://doi.org/10.1016/j.jht.2012.09.002

Chuang, L., Wu, C., \& Lin, K. (2012). Reliability, validity, and responsiveness of myotonometric measurement of muscle tone, elasticity, and stiffness in patients with stroke. Archives of Physical Medicine and Rehabilitation, 93(3), 532-540. http://doi.org/10.1016/j.apmr.2011.09.014

Cohen, J. A., Fischer, J. S., Bolibrush, D. M., Jak, A. J., Kniker, J. E., Mertz, L. A., ... Cutter, G. R. (2000). Intrarater and interrater reliability of the MS functional composite outcome measure. Neurology, 54(4), 802-806.

Collen, F. M., Wade, D. T., \& Bradshaw, C. M. (1990). Mobility after stroke: reliability of measures of impairment and disability. International Disability Studies, 12(1), 6-9.

Collin, C., \& Wade, D. (1990). Assessing motor impairment after stroke: a pilot reliability study. Journal of Neurology, Neurosurgery, and Psychiatry, 53(7), 576-579.

Combs, S. A., Kelly, S. P., Barton, R., Ivaska, M., \& Nowak, K. (2010). Effects of an intensive, task-specific rehabilitation program for individuals with chronic stroke: a case series. Disability and Rehabilitation, 32(8), 669-678. http://doi.org/10.3109/09638280903242716

Cook, D., \& Beckman, T. (2006). Current concepts in validity and reliability for psychometric instruments: theory and application. The American Journal of Medicine, 119(2), 166.e716. 
Cook, K., Teal, C., Bjorner, J., Cella, D., Chang, C. H., Crane, P., ... Reeve, B. (2007). IRT health outcomes data analysis project: an overview and summary. Quality of Life Research: An International Journal of Quality of Life Aspects of Treatment, Care and Rehabilitation, 16 Suppl 1, 121-132. http://doi.org/10.1007/s11136-007-9177-5

Cronbach, L., \& Meehl, P. (1955). Construct validity in psychological tests. Psychological Bulletin, 52(4), 281-302.

Crow, J. L., \& Harmeling-van der Wel, B. C. (2008). Hierarchical properties of the motor function sections of the Fugl-Meyer assessment scale for people after stroke: a retrospective study. Physical Therapy, 88(12), 1554-1567. http://doi.org/10.2522/ptj.20070186

Cutkosky, M., \& Wright, P. (1986). Modeling Manufacturing Grips and Correlation with the Design of Robotic Hands. Proceedings of IEEE International Conference on Robotics and Automation, 1533-39.

Dahl, A. E., Askim, T., Stock, R., Langørgen, E., Lydersen, S., \& Indredavik, B. (2008). Shortand long-term outcome of constraint-induced movement therapy after stroke: a randomized controlled feasibility trial. Clinical Rehabilitation, 22(5), 436-447. http://doi.org/10.1177/0269215507084581

Dalbeth, N., Collis, J., Gregory, K., Clark, B., Robinson, E., \& McQueen, F. M. (2007). Tophaceous joint disease strongly predicts hand function in patients with gout. Rheumatology, 46(12), 1804-1807. http://doi.org/10.1093/rheumatology/kem246

Daley, K., Mayo, N., \& Wood-Dauphinée, S. (1999). Reliability of scores on the Stroke Rehabilitation Assessment of Movement (STREAM) measure. Physical Therapy, 79(1), 8-19; quiz 20-23. 
Daly, J. J., Hogan, N., Perepezko, E. M., Krebs, H. I., Rogers, J. M., Goyal, K. S., .. Ruff, R. L. (2005). Response to upper-limb robotics and functional neuromuscular stimulation following stroke. Journal of Rehabilitation Research and Development, 42(6), 723-736.

Daly, J. J., \& Ruff, R. L. (2007). Construction of efficacious gait and upper limb functional interventions based on brain plasticity evidence and model-based measures for stroke patients. TheScientificWorldJournal, 7, 2031-2045. http://doi.org/10.1100/tsw.2007.299

Dean, C., \& Mackey, F. (1992). Motor assessment scale scores as a measure of rehabilitation outcome following stroke. Australian Journal of Physiotherapy, 38, 31-35.

Demeurisse, G., Demol, O., \& Robaye, E. (1979). Motor evaluation in vascular hemiplegia. Bruxelles Médical, 59(2), 87-94.

Demeurisse, G., Demol, O., \& Robaye, E. (1980). Motor evaluation in vascular hemiplegia. European Neurology, 19(6), 382-389.

Desrosiers, J., Bravo, G., Hébert, R., \& Dutil, E. (1995). Normative data for grip strength of elderly men and women. The American Journal of Occupational Therapy: Official Publication of the American Occupational Therapy Association, 49(7), 637-644.

Desrosiers, J., Hébert, R., Bravo, G., \& Dutil, E. (1995). Upper extremity performance test for the elderly (TEMPA): normative data and correlates with sensorimotor parameters. Test d'Evaluation des Membres Supérieurs de Personnes Agées. Archives of Physical Medicine and Rehabilitation, 76(12), 1125-1129.

Dettmann, M. A., Linder, M. T., \& Sepic, S. B. (1987). Relationships among walking performance, postural stability, and functional assessments of the hemiplegic patient. American Journal of Physical Medicine, 66(2), 77-90. 
DeVellis, R. (2012). Scale Development (3rd ed.). Chapel Hill, North Carolina: SAGE Publications Ltd.

Devraj, R., \& Wallace, L. S. (2013). Application of the content expert process to develop a clinically useful low-literacy Chronic Kidney Disease Self-Management Knowledge Tool (CKD-SMKT). Research in Social \& Administrative Pharmacy: RSAP, 9(5), 633-639. http://doi.org/10.1016/j.sapharm.2012.09.006

Doyle, P. J., McNeil, M. R., Bost, J. E., Ross, K. B., Wambaugh, J. L., Hula, W. D., \& Mikolic, J. M. (2007). The Burden of Stroke Scale (BOSS) provided valid, reliable, and responsive score estimates of functioning and well-being during the first year of recovery from stroke. Quality of Life Research: An International Journal of Quality of Life Aspects of Treatment, Care and Rehabilitation, 16(8), 1389-1398. http://doi.org/10.1007/s11136$007-9247-8$

Drussell, R. D. (1959). Relationship of Minnesota Rate of Manipulation Test with the industrial work performance of the adult cerebral palsied. The American Journal of Occupational Therapy: Official Publication of the American Occupational Therapy Association, 13(2, Part 2), 93-96 passim.

Duncan, P. W., Goldstein, L. B., Matchar, D., Divine, G. W., \& Feussner, J. (1992). Measurement of motor recovery after stroke. Outcome assessment and sample size requirements. Stroke; a Journal of Cerebral Circulation, 23(8), 1084-1089.

Duncan, P. W., Lai, S., Tyler, D., Perera, S., Reker, D., \& Studenski, S. (2002). Evaluation of proxy responses to the Stroke Impact Scale. Stroke; a Journal of Cerebral Circulation, 33(11), 2593-2599. 
Duncan, P. W., Propst, M., \& Nelson, S. G. (1983). Reliability of the Fugl-Meyer assessment of sensorimotor recovery following cerebrovascular accident. Physical Therapy, 63(10), $1606-1610$.

Duncan, P. W., Wallace, D., Lai, S. M., Johnson, D., Embretson, S., \& Laster, L. J. (1999). The stroke impact scale version 2.0. Evaluation of reliability, validity, and sensitivity to change. Stroke; a Journal of Cerebral Circulation, 30(10), 2131-2140.

Earhart, G. M., Cavanaugh, J. T., Ellis, T., Ford, M. P., Foreman, K. B., \& Dibble, L. (2011). The 9-hole PEG test of upper extremity function: average values, test-retest reliability, and factors contributing to performance in people with Parkinson disease. Journal of Neurologic Physical Therapy: JNPT, 35(4), 157-163.

http://doi.org/10.1097/NPT.0b013e318235da08

Endres, M., Nyary, I., Banhidi, M., \& Deak, G. (1990). Stroke rehabilitation: A method and evaluation. International Journal of Rehabilitation Research. Internationale Zeitschrift Für Rehabilitationsforschung. Revue Internationale De Recherches De Réadaptation, 13, $225-236$.

English, C. K., Hillier, S. L., Stiller, K., \& Warden-Flood, A. (2006). The sensitivity of three commonly used outcome measures to detect change amongst patients receiving inpatient rehabilitation following stroke. Clinical Rehabilitation, 20(1), 52-55.

Erasmus, L. P., Sarno, S., Albrecht, H., Schwecht, M., Pöllmann, W., \& König, N. (2001). Measurement of ataxic symptoms with a graphic tablet: standard values in controls and validity in Multiple Sclerosis patients. Journal of Neuroscience Methods, 108(1), 25-37. 
Erkinjuntti, T., Sulkava, R., Wikström, J., \& Autio, L. (1987). Short Portable Mental Status Questionnaire as a screening test for dementia and delirium among the elderly. Journal of the American Geriatrics Society, 35(5), 412-416.

Ferreiro, K., dos Santos, R., \& Conforto, A. (2010). Psychometric properties of the Portuguese version of the Jebsen-Taylor test for adults with mild hemiparesis. Revista Brasileira de Fisioterapia (Brazilian Journal of Physiotherapy), 14(5), 377-81.

Feys, P., Duportail, M., Kos, D., Van Asch, P., \& Ketelaer, P. (2002). Validity of the TEMPA for the measurement of upper limb function in multiple sclerosis. Clinical Rehabilitation, $16(2), 166-173$.

Feys, P., Romberg, A., Ruutiainen, J., \& Ketelaer, P. (2004). Interference of Upper Limb Tremor on Daily Life Activities in People with Multiple Sclerosis. Occupational Therapy in Health Care, 17(3-4), 81-95. http://doi.org/10.1080/J003v17n03_06

Finch, E., Brooks, D., Stratford, P., \& Mayo, N. (2002). Physical Outcome Measures: A guide to enhance physical outcome meaures. Ontario, Canada: Lippincott, Williams \& Wilkins.

Fong, K., Ng, B., Chan, D., Chan, E., Ma, D., Au, B., ... Chan, V. (2004). Development of the Hong Kong Version of the Functional Test for the Hemiplegic Upper Extremity (FTHUE-HK). Hong Kong Journal of Occupational Therapy, 14(1), 21-29. http://doi.org/10.1016/S1569-1861(09)70025-7

Freitas, S., Simões, M. R., Marôco, J., Alves, L., \& Santana, I. (2012). Construct Validity of the Montreal Cognitive Assessment (MoCA). Journal of the International Neuropsychological Society: JINS, 18(2), 242-250. 
Fritz, S. L., Blanton, S., Uswatte, G., Taub, E., \& Wolf, S. L. (2009). Minimal detectable change scores for the Wolf Motor Function Test. Neurorehabilitation and Neural Repair, 23(7), 662-667. http://doi.org/10.1177/1545968309335975

Gershon, R. C., Lai, J. S., Bode, R., Choi, S., Moy, C., Bleck, T., ... Cella, D. (2012). NeuroQOL: quality of life item banks for adults with neurological disorders: item development and calibrations based upon clinical and general population testing. Quality of Life Research: An International Journal of Quality of Life Aspects of Treatment, Care and Rehabilitation, 21(3), 475-486. http://doi.org/10.1007/s11136-011-9958-8

Gershon, R. C., Rothrock, N., Hanrahan, R., Bass, M., \& Cella, D. (2010). The use of PROMIS and assessment center to deliver patient-reported outcome measures in clinical research. Journal of Applied Measurement, 11(3), 304-314.

Gladstone, D. J., Danells, C. J., \& Black, S. E. (2002). The fugl-meyer assessment of motor recovery after stroke: a critical review of its measurement properties. Neurorehabilitation and Neural Repair, 16(3), 232-240.

Golafshani, N. (2003). Understanding Reliability and Validity in Qualitative Research. The Qualitative Report, 8(4), 597-607.

Gummesson, C., Atroshi, I., \& Ekdahl, C. (2003). The disabilities of the arm, shoulder and hand (DASH) outcome questionnaire: longitudinal construct validity and measuring self-rated health change after surgery. BMC Musculoskeletal Disorders, 4, 11. http://doi.org/10.1186/1471-2474-4-11

Gurcay, E., Bal, A., \& Cakci, A. (2009). Health-related quality of life in first-ever stroke patients. Annals of Saudi Medicine, 29(1), 36-40. 
Gustafsson, L. A., Turpin, M. J., \& Dorman, C. M. (2010). Clinical utility of the Chedoke Arm and Hand Activity Inventory for stroke rehabilitation. Canadian Journal of Occupational Therapy. Revue Canadienne D'ergothérapie, 77(3), 167-173.

Hackel, M. E., Wolfe, G. A., Bang, S. M., \& Canfield, J. S. (1992). Changes in hand function in the aging adult as determined by the Jebsen Test of Hand Function. Physical Therapy, $72(5), 373-377$.

Hammer, A. M., \& Lindmark, B. (2010). Responsiveness and validity of the Motor Activity Log in patients during the subacute phase after stroke. Disability and Rehabilitation, 32(14), 1184-1193. http://doi.org/10.3109/09638280903437253

Harris, J. E., Eng, J. J., Miller, W. C., \& Dawson, A. S. (2009). A self-administered Graded Repetitive Arm Supplementary Program (GRASP) improves arm function during inpatient stroke rehabilitation: a multi-site randomized controlled trial. Stroke; a Journal of Cerebral Circulation, 40(6), 2123-2128. http://doi.org/10.1161/STROKEAHA.108.544585

Hays, R. D., Spritzer, K. L., Amtmann, D., Lai, J.S., Dewitt, E. M., Rothrock, N., ... Krishnan, E. (2013). Upper-extremity and mobility subdomains from the Patient-Reported Outcomes Measurement Information System (PROMIS) adult physical functioning item bank. Archives of Physical Medicine and Rehabilitation, 94(11), 2291-2296. http://doi.org/10.1016/j.apmr.2013.05.014

Heller, A., Wade, D. T., Wood, V. A., Sunderland, A., Hewer, R. L., \& Ward, E. (1987). Arm function after stroke: measurement and recovery over the first three months. Journal of Neurology, Neurosurgery, and Psychiatry, 50(6), 714-719. 
Hesse, S., Werner, C., Pohl, M., Rueckriem, S., Mehrholz, J., \& Lingnau, M. L. (2005).

Computerized arm training improves the motor control of the severely affected arm after stroke: a single-blinded randomized trial in two centers. Stroke; a Journal of Cerebral Circulation, 36(9), 1960-1966. http://doi.org/10.1161/01.STR.0000177865.37334.ce

Higgins, J., Mayo, N. E., Desrosiers, J., Salbach, N. M., \& Ahmed, S. (2005). Upper-limb function and recovery in the acute phase poststroke. The Journal of Rehabilitation Research and Development, 42(1), 65. http://doi.org/10.1682/JRRD.2003.10.0156

Higgins, J., Salbach, N. M., Wood-Dauphinee, S., Richards, C. L., Côté, R., \& Mayo, N. E. (2006). The effect of a task-oriented intervention on arm function in people with stroke: a randomized controlled trial. Clinical Rehabilitation, 20(4), 296-310.

Hijmans, J. M., Hale, L. A., Satherley, J. A., McMillan, N. J., \& King, M. J. (2011). Bilateral upper-limb rehabilitation after stroke using a movement-based game controller. Journal of Rehabilitation Research and Development, 48(8), 1005-1013.

Hsieh, Y., Wu, C., Lin, K., Chang, Y., Chen, C., \& Liu, J. (2009). Responsiveness and validity of three outcome measures of motor function after stroke rehabilitation. Stroke; a Journal of Cerebral Circulation, 40(4), 1386-1391. http://doi.org/10.1161/STROKEAHA.108.530584

Hsueh, I.P., Hsu, M.J., Sheu, C.F., Lee, S., Hsieh, C.L., \& Lin, J.H. (2008). Psychometric comparisons of 2 versions of the Fugl-Meyer Motor Scale and 2 versions of the Stroke Rehabilitation Assessment of Movement. Neurorehabilitation and Neural Repair, 22(6), 737-744. http://doi.org/10.1177/1545968308315999

Hsueh, I.P., Wang, C.H., Sheu, C.F., \& Hsieh, C.L. (2003). Comparison of psychometric properties of three mobility measures for patients with stroke. Stroke; a Journal of 
Cerebral Circulation, 34(7), 1741-1745.

http://doi.org/10.1161/01.STR.0000075295.45185.D4

Hsu, J. E., Nacke, E., Park, M. J., Sennett, B. J., \& Huffman, G. R. (2010). The Disabilities of the Arm, Shoulder, and Hand questionnaire in intercollegiate athletes: validity limited by ceiling effect. Journal of Shoulder and Elbow Surgery / American Shoulder and Elbow Surgeons ... [et Al.], 19(3), 349-354. http://doi.org/10.1016/j.jse.2009.11.006

Huang, Y., Wu, C., Hsieh, Y., \& Lin, K. (2010). Predictors of change in quality of life after distributed constraint-induced therapy in patients with chronic stroke.

Neurorehabilitation and Neural Repair, 24(6), 559-566.

http://doi.org/10.1177/1545968309358074

Hudak, P. L., Amadio, P. C., \& Bombardier, C. (1996). Development of an upper extremity outcome measure: the DASH (disabilities of the arm, shoulder and hand) [corrected]. The Upper Extremity Collaborative Group (UECG). American Journal of Industrial Medicine, 29(6), 602-608. http://doi.org/10.1002/(SICI)10970274(199606)29:6<602::AID-AJIM4>3.0.CO;2-L

Hughes, A. M., Freeman, C. T., Burridge, J. H., Chappell, P. H., Lewin, P. L., \& Rogers, E. (2009). Feasibility of iterative learning control mediated by functional electrical stimulation for reaching after stroke. Neurorehabilitation and Neural Repair, 23(6), 559568. http://doi.org/10.1177/1545968308328718

Huisstede, B. M. A., Feleus, A., Bierma-Zeinstra, S. M., Verhaar, J. A., \& Koes, B. W. (2009). Is the disability of arm, shoulder, and hand questionnaire (DASH) also valid and responsive in patients with neck complaints. Spine, 34(4), E130-138.

http://doi.org/10.1097/BRS.0b013e318195a28b 
Humphry, R., Jewell, K., \& Rosenberger, R. C. (1995). Development of in-hand manipulation and relationship with activities. The American Journal of Occupational Therapy: Official Publication of the American Occupational Therapy Association, 49(8), 763-771.

Hung, M., Clegg, D. O., Greene, T., \& Saltzman, C. L. (2011). Evaluation of the PROMIS physical function item bank in orthopaedic patients. Journal of Orthopaedic Research: Official Publication of the Orthopaedic Research Society, 29(6), 947-953. http://doi.org/10.1002/jor.21308

Hwang, E. J., Hauschild, M., Wilke, M., \& Andersen, R. A. (2012). Inactivation of the parietal reach region causes optic ataxia, impairing reaches but not saccades. Neuron, 76(5), 1021-1029. http://doi.org/10.1016/j.neuron.2012.10.030

Ietswaart, M., Johnston, M., Dijkerman, H. C., Joice, S., Scott, C. L., MacWalter, R. S., \& Hamilton, S. J. C. (2011). Mental practice with motor imagery in stroke recovery: randomized controlled trial of efficacy. Brain: A Journal of Neurology, 134(Pt 5), 13731386. http://doi.org/10.1093/brain/awr077

Imaeda, T., Uchiyama, S., Wada, T., Okinaga, S., Sawaizumi, T., Omokawa, S., ... Clinical Outcomes Committee of the Japanese Orthopaedic Association and the Functional Evaluation Committee of the Japanese Society for Surgery of the Hand. (2010). Reliability, validity, and responsiveness of the Japanese version of the Patient-Rated Wrist Evaluation. Journal of Orthopaedic Science: Official Journal of the Japanese Orthopaedic Association, 15(4), 509-517. http://doi.org/10.1007/s00776-010-1477-x

Jacob-Lloyd, H., \& Dunn, O. (2005). Effective measurement of the functional progress of stroke clients. The British Journal of Occupational Therapy, 68(6), 253-59. 
Jebsen, R. H., Taylor, N., Trieschmann, R. B., Trotter, M. J., \& Howard, L. A. (1969). An objective and standardized test of hand function. Archives of Physical Medicine and Rehabilitation, 50(6), 311-319.

Joint Committee on Testing Practices. (2004). Code of fair testing practices in education. National Council on Measurement in Education. Retrieved from http://www.apa.org/science/programs/testing/fair-testing.pdf

Jones, L. A., \& Lederman, S. J. (2006). Human hand function. Oxford; New York: Oxford University Press.

Kandel, E. R., Schwartz, J. H., Jessell, T. M., Siegelbaum, S. A., \& Hudspeth, A. J. (Eds.). (2012). Principles of Neural Science, Fifth Edition (Principles of Neural Science (5th ed.). McGraw-Hill Professional.

Kellor, M., Frost, J., Silberberg, N., Iversen, I., \& Cummings, R. (1971). Hand strength and dexterity. The American Journal of Occupational Therapy: Official Publication of the American Occupational Therapy Association, 25(2), 77-83.

Kennedy, C. A., Beaton, D. E., Smith, P., Van Eerd, D., Tang, K., Inrig, T., ... Couban, R. (2013). Measurement properties of the QuickDASH (disabilities of the arm, shoulder and hand) outcome measure and cross-cultural adaptations of the QuickDASH: a systematic review. Quality of Life Research: An International Journal of Quality of Life Aspects of Treatment, Care and Rehabilitation, 22(9), 2509-2547. http://doi.org/10.1007/s11136013-0362-4

Ketterlin-Geller, L. R., Yovanoff, P., Jung, E., Liu, K., \& Geller, J. (2013). Construct Definition Using Cognitively Based Evidence: A Framework for Practice. Educational Assessment, 18(2), 122-146. http://doi.org/10.1080/10627197.2013.790207 
Knutson, J. S., Harley, M. Y., Hisel, T. Z., Hogan, S. D., Maloney, M. M., \& Chae, J. (2012). Contralaterally controlled functional electrical stimulation for upper extremity hemiplegia: an early-phase randomized clinical trial in subacute stroke patients. Neurorehabilitation and Neural Repair, 26(3), 239-246. http://doi.org/10.1177/1545968311419301

Kopp, B., Kunkel, A., Flor, H., Platz, T., Rose, U., Mauritz, K. H., ... Taub, E. (1997a). The Arm Motor Ability Test: reliability, validity, and sensitivity to change of an instrument for assessing disabilities in activities of daily living. Archives of Physical Medicine and Rehabilitation, 78(6), 615-620.

Kopp, B., Kunkel, A., Flor, H., Platz, T., Rose, U., Mauritz, K. H., ... Taub, E. (1997b). The Arm Motor Ability Test: reliability, validity, and sensitivity to change of an instrument for assessing disabilities in activities of daily living. Archives of Physical Medicine and Rehabilitation, 78(6), 615-620.

Kraft, G. H., Amtmann, D., Bennett, S. E., Finlayson, M., Sutliff, M. H., Tullman, M., ... Rabinowicz, A. L. (2014). Assessment of upper extremity function in multiple sclerosis: review and opinion. Postgraduate Medicine, 126(5), 102-108. http://doi.org/10.3810/pgm.2014.09.2803

Krebs, H. I., Mernoff, S., Fasoli, S. E., Hughes, R., Stein, J., \& Hogan, N. (2008). A comparison of functional and impairment-based robotic training in severe to moderate chronic stroke: a pilot study. NeuroRehabilitation, 23(1), 81-87.

Krosnick, J., Holbrook, A., Berent, M., Carson, R., Hanemann, W., \& Kopp, R. (2002). The impact of "no opinion" response options on data quality: Non-attitude reduction or an invitation to satisfice? Public Opinion Quarterly, 66(3), 371-403. 
Kunkel, A., Kopp, B., Müller, G., Villringer, K., Villringer, A., Taub, E., \& Flor, H. (1999). Constraint-induced movement therapy for motor recovery in chronic stroke patients. Archives of Physical Medicine and Rehabilitation, 80(6), 624-628.

Kurtaiş, Y., Küçükdeveci, A., Elhan, A., Yilmaz, A., Kalli, T., Tur, B. S., \& Tennant, A. (2009). Psychometric properties of the Rivermead Motor Assessment: its utility in stroke. Journal of Rehabilitation Medicine, 41(13), 1055-1061. http://doi.org/10.2340/16501977-0463

Lamers, I., Kerkhofs, L., Raats, J., Kos, D., Van Wijmeersch, B., \& Feys, P. (2013). Perceived and actual arm performance in multiple sclerosis: relationship with clinical tests according to hand dominance. Multiple Sclerosis (Houndmills, Basingstoke, England), 19(10), 1341-1348. http://doi.org/10.1177/1352458513475832

Lamers, I., Timmermans, A., Kerkhofs, L., Severijns, D., Van Wijmeersch, B., \& Feys, P. (2013). Self-reported use of the upper limbs related to clinical tests in persons with multiple sclerosis. Disability and Rehabilitation, 35(23), 2016-2020. http://doi.org/10.3109/09638288.2013.771703

Lang, C. E., Edwards, D. F., Birkenmeier, R. L., \& Dromerick, A. W. (2008). Estimating minimal clinically important differences of upper-extremity measures early after stroke. Archives of Physical Medicine and Rehabilitation, 89(9), 1693-1700. http://doi.org/10.1016/j.apmr.2008.02.022

Lang, C. E., Wagner, J. M., Dromerick, A. W., \& Edwards, D. F. (2006). Measurement of upperextremity function early after stroke: properties of the action research arm test. Archives of Physical Medicine and Rehabilitation, 87(12), 1605-1610. http://doi.org/10.1016/j.apmr.2006.09.003 
Lannin, N., McCluskey, A., Cusick, A., Ashford, S., \& Ross, L. (2010). Measuring function in everyday life: enhancing the Disabilities of the Arm Shoulder Hand questionnaire for use post-stroke. Presented at the World Federation of Occcupational Therapy, Santiago, Chile.

Lawshe, C. (1975). A quantitative approach to content validity. Personnel Psychology, 28, 563575. http://doi.org/10.1111/j.1744-6570.1975.tb01393.x

Lehman, L. A., Woodbury, M., Shechtman, O., Wang, Y. C., Pomeranz, J., Gray, D. B., \& Velozo, C. A. (2011). Development of an item bank for a computerised adaptive test of upper-extremity function. Disability and Rehabilitation, 33(21-22), 2092-2104. http://doi.org/10.3109/09638288.2011.560336

Lemmens, R. J. M., Timmermans, A. A. A., Janssen-Potten, Y. J. M., Smeets, R. J. E. M., \& Seelen, H. A. M. (2012). Valid and reliable instruments for arm-hand assessment at ICF activity level in persons with hemiplegia: a systematic review. BMC Neurology, 12, 21. http://doi.org/10.1186/1471-2377-12-21

Levy, R., Ruland, S., Weinand, M., Lowry, D., Dafer, R., \& Bakay, R. (2008). Cortical stimulation for the rehabilitation of patients with hemiparetic stroke: a multicenter feasibility study of safety and efficacy. Journal of Neurosurgery, 108(4), 707-714. http://doi.org/10.3171/JNS/2008/108/4/0707

Lincoln, N., \& Leadbitter, D. (1979). Assessment of motor function in stroke patients. Physiotherapy, 65(2), 48-51.

Lin, J.H., Hsueh, I.P., Sheu, C.F., \& Hsieh, C.L. (2004). Psychometric properties of the sensory scale of the Fugl-Meyer Assessment in stroke patients. Clinical Rehabilitation, 18(4), 391-397. 
Lin, J.H., Hsu, M.J., Sheu, C.F., Wu, T.S., Lin, R.T., Chen, C.H., \& Hsieh, C.L. (2009). Psychometric comparisons of 4 measures for assessing upper-extremity function in people with stroke. Physical Therapy, 89(8), 840-850. http://doi.org/10.2522/ptj.20080285

Lin, K.C., Fu, T., Wu, C.Y., Hsieh, Y.W., Chen, C.L., \& Lee, P.C. (2010). Psychometric comparisons of the Stroke Impact Scale 3.0 and Stroke-Specific Quality of Life Scale. Quality of Life Research: An International Journal of Quality of Life Aspects of Treatment, Care and Rehabilitation, 19(3), 435-443. http://doi.org/10.1007/s11136-010$9597-5$

Lin, K., Chang, Y., Wu, C., \& Chen, Y. (2009). Effects of constraint-induced therapy versus bilateral arm training on motor performance, daily functions, and quality of life in stroke survivors. Neurorehabilitation and Neural Repair, 23(5), 441-448. http://doi.org/10.1177/1545968308328719

Lin, K., Chuang, L., Wu, C., Hsieh, Y., \& Chang, W. (2010). Responsiveness and validity of three dexterous function measures in stroke rehabilitation. Journal of Rehabilitation Research and Development, 47(6), 563-571.

Lin, K.C., Huang, Y.H., Hsieh, Y.W., \& Wu, C.Y. (2009). Potential predictors of motor and functional outcomes after distributed constraint-induced therapy for patients with stroke. Neurorehabilitation and Neural Repair, 23(4), 336-342. http://doi.org/10.1177/1545968308321773

Lin, K.C., Wu, C.Y., Wei, T.H., Lee, C.Y., \& Liu, J. S. (2007). Effects of modified constraintinduced movement therapy on reach-to-grasp movements and functional performance 
after chronic stroke: a randomized controlled study. Clinical Rehabilitation, 21(12), 1075-1086. http://doi.org/10.1177/0269215507079843

Lin, K., Fu, T., Wu, C., Wang, Y., Liu, J., Hsieh, C., \& Lin, S. (2010). Minimal detectable change and clinically important difference of the Stroke Impact Scale in stroke patients. Neurorehabilitation and Neural Repair, 24(5), 486-492. http://doi.org/10.1177/1545968309356295

Lin, K., Wu, C., Liu, J., Chen, Y., \& Hsu, C. (2009). Constraint-induced therapy versus dosematched control intervention to improve motor ability, basic/extended daily functions, and quality of life in stroke. Neurorehabilitation and Neural Repair, 23(2), 160-165. http://doi.org/10.1177/1545968308320642

Livingston, S., \& Zieky, M. (1982). Passing Scores: A Manual for Setting Standards of Performance on Educational and Occupational Tests. Educational Testing Service, Princeton, NJ. Retrieved from https://www.ets.org/Media/Research/pdf/passing_scores.pdf

Lundy-Ekman, L. (2012). Neuroscience: Fundamentals for Rehabilitation, 4e (4th ed.). Saunders.

Lyle, R. C. (1981). A performance test for assessment of upper limb function in physical rehabilitation treatment and research: International Journal of Rehabilitation Research, 4(4), 483-492. http://doi.org/10.1097/00004356-198112000-00001

MacDermid, J. C., Wessel, J., Humphrey, R., Ross, D., \& Roth, J. H. (2007). Validity of selfreport measures of pain and disability for persons who have undergone arthroplasty for osteoarthritis of the carpometacarpal joint of the hand. Osteoarthritis and Cartilage / 
OARS, Osteoarthritis Research Society, 15(5), 524-530.

http://doi.org/10.1016/j.joca.2006.10.018

Malouin, F., Pichard, L., Bonneau, C., Durand, A., \& Corriveau, D. (1994). Evaluating motor recovery early after stroke: comparison of the Fugl-Meyer Assessment and the Motor Assessment Scale. Archives of Physical Medicine and Rehabilitation, 75(11), 1206-1212.

Mao, H. F., Hsueh, I. P., Tang, P. F., Sheu, C. F., \& Hsieh, C. L. (2002). Analysis and comparison of the psychometric properties of three balance measures for stroke patients. Stroke; a Journal of Cerebral Circulation, 33(4), 1022-1027.

Marino, R. J., Kern, S. B., Leiby, B., Schmidt-Read, M., \& Mulcahey, M. J. (2015). Reliability and validity of the capabilities of upper extremity test (CUE-T) in subjects with chronic spinal cord injury. The Journal of Spinal Cord Medicine, 38(4), 498-504.

http://doi.org/10.1179/2045772314Y.0000000272

Marshall, M. (1996). Sampling for qualitative research. Family Practice, 13, 522-525.

Masiero, S., Celia, A., Rosati, G., \& Armani, M. (2007). Robotic-assisted rehabilitation of the upper limb after acute stroke. Archives of Physical Medicine and Rehabilitation, 88(2), 142-149. http://doi.org/10.1016/j.apmr.2006.10.032

Mathiowetz, V., Kashman, N., Volland, G., Weber, K., Dowe, M., \& Rogers, S. (1985). Grip and pinch strength: normative data for adults. Archives of Physical Medicine and Rehabilitation, 66(2), 69-74.

McDonnell, M. N., Hillier, S. L., Ridding, M. C., \& Miles, T. S. (2006). Impairments in precision grip correlate with functional measures in adult hemiplegia. Clinical Neurophysiology: Official Journal of the International Federation of Clinical Neurophysiology, 117(7), 1474-1480. http://doi.org/10.1016/j.clinph.2006.02.027 
McDowell, I. (2006). Measuring Health:A Guide to Rating Scales and Questionnaires (3rd ed.). Oxford University Press, USA.

Mehta, S., Macdermid, J. C., Carlesso, L. C., \& McPhee, C. (2010). Concurrent validation of the DASH and the QuickDASH in comparison to neck-specific scales in patients with neck pain. Spine, 35(24), 2150-2156. http://doi.org/10.1097/BRS.0b013e3181c85151

Mesquita, R., Janssen, D. J. A., Wouters, E. F. M., Schols, J. M. G. A., Pitta, F., \& Spruit, M. A. (2013). Within-Day Test-Retest Reliability of the Timed Up \& Go Test in Patients With Advanced Chronic Organ Failure. Archives of Physical Medicine and Rehabilitation. http://doi.org/10.1016/j.apmr.2013.03.024

Messick, S. (1989). Meaning and Values in Test Validation: The Science and Ethics of Assessment. Educational Researcher, 18(2), 5-11.

Messick, S. (1994). The Interplay of Evidence and Consequences in the Validation of Performance Assessments. Educational Researcher, 23(2), 13-23.

Metcalf, C., Adams, J., Burridge, J., Yule, V., \& Chappell, P. (2007). A review of clinical upper limb assessments within the framework of the WHO ICF. Musculoskeletal Care, 5(3), 160-173. http://doi.org/10.1002/msc.108

Milot, M.H., Spencer, S. J., Chan, V., Allington, J. P., Klein, J., Chou, C., ... Cramer, S. C. (2014). Corticospinal excitability as a predictor of functional gains at the affected upper limb following robotic training in chronic stroke survivors. Neurorehabilitation and Neural Repair, 28(9), 819-827. http://doi.org/10.1177/1545968314527351

Mislevy, R. J. (2007). Validity by Design. Educational Researcher, 36(8), 463-469. 
Mislevy, R. J. (2011, July). Evidence-centered design for simulation-based assessment. National Center for Rearch on Evaluation, Standards and Student Testing, Center for the study of evaluation, Graduate School of Education and Information Studies, UCLA, Los Angeles.

Mislevy, R. J., Almond, R. G., \& Lukas, J. F. (2003, July). A Brief Introduction to Evidencecentered Design. Educational Testing Service, Princeton, NJ.

Miyamoto, S., Kondo, T., Suzukamo, Y., Michimata, A., \& Izumi, S.I. (2009). Reliability and validity of the Manual Function Test in patients with stroke. American Journal of Physical Medicine \& Rehabilitation / Association of Academic Physiatrists, 88(3), 247255. http://doi.org/10.1097/PHM.0b013e3181951133

Moher, D., Liberati, A., Tetzlaff, J., Altman, D. G., \& PRISMA Group. (2009). Preferred reporting items for systematic reviews and meta-analyses: the PRISMA statement. PLoS Medicine, 6(7), e1000097. http://doi.org/10.1371/journal.pmed.1000097

Morgan, S. J., Amtmann, D., Abrahamson, D. C., Kajlich, A. J., \& Hafner, B. J. (2014). Use of cognitive interviews in the development of the PLUS-M item bank. Quality of Life Research: An International Journal of Quality of Life Aspects of Treatment, Care and Rehabilitation, 23(6), 1767-1775. http://doi.org/10.1007/s11136-013-0618-z

Morris, D., Uswatte, G., Crago, J., Cook, E. W., 3rd, \& Taub, E. (2001). The reliability of the wolf motor function test for assessing upper extremity function after stroke. Archives of Physical Medicine and Rehabilitation, 82(6), 750-755. http://doi.org/10.1053/apmr.2001.23183

Morris, J. H., \& Van Wijck, F. (2012). Responses of the less affected arm to bilateral upper limb task training in early rehabilitation after stroke: a randomized controlled trial. Archives of 
Physical Medicine and Rehabilitation, 93(7), 1129-1137.

http://doi.org/10.1016/j.apmr.2012.02.025

Morris, J. H., van Wijck, F., Joice, S., Ogston, S. A., Cole, I., \& MacWalter, R. S. (2008). A comparison of bilateral and unilateral upper-limb task training in early poststroke rehabilitation: a randomized controlled trial. Archives of Physical Medicine and Rehabilitation, 89(7), 1237-1245. http://doi.org/10.1016/j.apmr.2007.11.039

Mulcahey, M., Smith, B., \& Betz, R. (2004). Psychometric Rigor of the Grasp and Release Test for Measuring Funcitonal Limitation of Persons with Tetraplegia: A Preliminary analysis. The Journal of Spinal Cord Medicine, 27(1), 41-46.

Napier, J., \& Tuttle, R. H. (1993). Hands (Rev Sub edition). Princeton, N.J: Princeton University Press.

Nasreddine, Z. S., Phillips, N. A., Bédirian, V., Charbonneau, S., Whitehead, V., Collin, I., ... Chertkow, H. (2005). The Montreal Cognitive Assessment, MoCA: a brief screening tool for mild cognitive impairment. Journal of the American Geriatrics Society, 53(4), 695699.

Nesselroade, J. R., Gerstorf, D., Hardy, S. A., \& Ram, N. (2007). Idiographic Filters for Psychological Constructs. Measurement: Interdisciplinary Research and Perspectives, 5(4), 217-235.

Netemeyer, R., Bearden, W., \& Sharma, S. (2003). Scaling Procedures: Issues and Applications (1 edition). Thousand Oaks, CA: SAGE Publications, Inc.

NIH Consensus Development Panel on Rehabilitation of Persons With Traumatic Brain Injury. (1999). Rehabilitation of persons with traumatic brain injury. JAMA, 282(10), 974-983. http://doi.org/10.1001/jama.282.10.974 
Nijland, R., van Wegen, E., Verbunt, J., van Wijk, R., van Kordelaar, J., \& Kwakkel, G. (2010). A comparison of two validated tests for upper limb function after stroke: The Wolf Motor Function Test and the Action Research Arm Test. Journal of Rehabilitation Medicine, 42(7), 694-696. http://doi.org/10.2340/16501977-0560

Ohn, S. H., Yoo, W. K., Kim, D. Y., Ahn, S., Jung, B., Choi, I., ... Jung, K. I. (2013). Measurement of synergy and spasticity during functional movement of the post-stoke hemiplegic upper limb. Journal of Electromyography and Kinesiology: Official Journal of the International Society of Electrophysiological Kinesiology, 23(2), 501-507. http://doi.org/10.1016/j.jelekin.2012.10.001

Oxford Grice, K., Vogel, K. A., Le, V., Mitchell, A., Muniz, S., \& Vollmer, M. A. (2003). Adult norms for a commercially available Nine Hole Peg Test for finger dexterity. The American Journal of Occupational Therapy: Official Publication of the American Occupational Therapy Association, 57(5), 570-573.

Page, S. J., Hade, E., \& Persch, A. (2015). Psychometrics of the wrist stability and hand mobility subscales of the Fugl-Meyer assessment in moderately impaired stroke. Physical Therapy, 95(1), 103-108. http://doi.org/10.2522/ptj.20130235

Page, S. J., Hermann, V. H., Levine, P. G., Lewis, E., Stein, J., \& DePeel, J. (2011). Portable neurorobotics for the severely affected arm in chronic stroke: a case study. Journal of Neurologic Physical Therapy: JNPT, 35(1), 41-46. http://doi.org/10.1097/NPT.0b013e31820c4b00

Page, S. J., Levine, P., \& Hade, E. (2012). Psychometric properties and administration of the wrist/hand subscales of the Fugl-Meyer Assessment in minimally impaired upper 
extremity hemiparesis in stroke. Archives of Physical Medicine and Rehabilitation, 93(12), 2373-2376.e5. http://doi.org/10.1016/j.apmr.2012.06.017

Pang, M. Y., Harris, J. E., \& Eng, J. J. (2006). A community-based upper-extremity group exercise program improves motor function and performance of functional activities in chronic stroke: a randomized controlled trial. Archives of Physical Medicine and Rehabilitation, 87(1), 1-9. http://doi.org/10.1016/j.apmr.2005.08.113

Patient-Centered Outcomes Research Institute. (2012, June 4). Preliminary draft methodology report. Retrieved January 5, 2016, from http://www.pcori.org/assets/Preliminary-DraftMethodology-Report.pdf

Pendleton, H., \& Schultz-Krohn, W. (2006). Pedretti's Occupational Therapy 6th (6th edition). Mosby Co.

Penta, M. (2015). ABILHAND - Presentation - Rehab-Scales.org. Retrieved October 22, 2015, from http://www.rehab-scales.org/abilhand-presentation.html\#development-of-theabilhand-scale-using-the-rasch-measurement-model

Penta, M., Tesio, L., Arnould, C., Zancan, A., \& Thonnard, J. L. (2001). The ABILHAND questionnaire as a measure of manual ability in chronic stroke patients: Rasch-based validation and relationship to upper limb impairment. Stroke; a Journal of Cerebral Circulation, 32(7), 1627-1634.

Penta, M., Thonnard, J. L., \& Tesio, L. (1998). ABILHAND: a Rasch-built measure of manual ability. Archives of Physical Medicine and Rehabilitation, 79(9), 1038-1042.

Peters, M. J. H., van Nes, S. I., Vanhoutte, E. K., Bakkers, M., van Doorn, P. A., Merkies, I. S. J., ... PeriNomS Study group. (2011). Revised normative values for grip strength with the 
Jamar dynamometer. Journal of the Peripheral Nervous System: JPNS, 16(1), 47-50. http://doi.org/10.1111/j.1529-8027.2011.00318.x

Pfeiffer, E. (1975). A short portable mental status questionnaire for the assessment of organic brain deficit in elderly patients. Journal of the American Geriatrics Society, 23(10), 433441.

Platz, T., Pinkowski, C., van Wijck, F., Kim, I. H., di Bella, P., \& Johnson, G. (2005). Reliability and validity of arm function assessment with standardized guidelines for the Fugl-Meyer Test, Action Research Arm Test and Box and Block Test: a multicentre study. Clinical Rehabilitation, 19(4), 404-411.

Polman, C. H., \& Rudick, R. A. (2010). The multiple sclerosis functional composite: a clinically meaningful measure of disability. Neurology, 74 Suppl 3, S8-15. http://doi.org/10.1212/WNL.0b013e3181dbb571

Pomeroy, V. M., Ward, N. S., Johansen-Berg, H., van Vliet, P., Burridge, J., Hunter, S. M., ... FAST INdICATE Investigators. (2014). FAST INdiCATE Trial protocol. Clinical efficacy of functional strength training for upper limb motor recovery early after stroke: neural correlates and prognostic indicators. International Journal of Stroke: Official Journal of the International Stroke Society, 9(2), 240-245. http://doi.org/10.1111/ijs.12179

Quatrano, L. A., \& Cruz, T. H. (2011). Future of outcomes measurement: impact on research in medical rehabilitation and neurologic populations. Archives of Physical Medicine and Rehabilitation, 92(10 Suppl), S7-11. http://doi.org/10.1016/j.apmr.2010.08.032

Rabadi, M. H., \& Rabadi, F. M. (2006). Comparison of the action research arm test and the FuglMeyer assessment as measures of upper-extremity motor weakness after stroke. Archives 
of Physical Medicine and Rehabilitation, 87(7), 962-966.

http://doi.org/10.1016/j.apmr.2006.02.036

Radomski, M., \& Trombly, C. A. (Eds.). (2007). Occupational Therapy for Physical Dysfunction (Sixth). Philadelphia: Lippincott Williams \& Wilkins.

Rallon, C. R., \& Chen, C. C. (2008). Relationship between performance-based and self-reported assessment of hand function. The American Journal of Occupational Therapy: Official Publication of the American Occupational Therapy Association, 62(5), 574-579.

Rapin, I., Tourk, L. M., \& Costa, L. D. (1966). Evaluation of the Purdue Pegboard as a screening test for brain damage. Developmental Medicine and Child Neurology, 8(1), 45-54.

Reinkensmeyer, D. J., Wolbrecht, E. T., Chan, V., Chou, C., Cramer, S. C., \& Bobrow, J. E. (2012). Comparison of three-dimensional, assist-as-needed robotic arm/hand movement training provided with Pneu-WREX to conventional tabletop therapy after chronic stroke. American Journal of Physical Medicine \& Rehabilitation / Association of Academic Physiatrists, 91(11 Suppl 3), S232-241. http://doi.org/10.1097/PHM.0b013e31826bce79

Rosati, G., Gallina, P., \& Masiero, S. (2007). Design, implementation and clinical tests of a wirebased robot for neurorehabilitation. IEEE Transactions on Neural Systems and Rehabilitation Engineering: A Publication of the IEEE Engineering in Medicine and Biology Society, 15(4), 560-569. http://doi.org/10.1109/TNSRE.2007.908560

Rosewilliam, S., Malhotra, S., Roffe, C., Jones, P., \& Pandyan, A. D. (2012). Can surface neuromuscular electrical stimulation of the wrist and hand combined with routine therapy facilitate recovery of arm function in patients with stroke? Archives of Physical Medicine and Rehabilitation, 93(10), 1715-1721.e1. http://doi.org/10.1016/j.apmr.2012.05.017 
Sabari, J. S., Lim, A. L., Velozo, C. A., Lehman, L., Kieran, O., \& Lai, J. S. (2005). Assessing arm and hand function after stroke: a validity test of the hierarchical scoring system used in the motor assessment scale for stroke. Archives of Physical Medicine and Rehabilitation, 86(8), 1609-1615. http://doi.org/10.1016/j.apmr.2004.12.028

Sackley, C. M., \& Lincoln, N. B. (1990). The verbal administration of the gross function scale of the Rivermead Motor Assessment. Clinical Rehabilitation, 4(4), 301-303. http://doi.org/10.1177/026921559000400409

Sadler, J. Z. (2003). Descriptions and Prescriptions: Values, Mental Disorders, and the DSMs. Baltimore, MD. The John Hopkins University Press.

Sawaki, L., Butler, A. J., Leng, X., Wassenaar, P. A., Mohammad, Y. M., Blanton, S., ... Wittenberg, G. F. (2008). Constraint-induced movement therapy results in increased motor map area in subjects 3 to 9 months after stroke. Neurorehabilitation and Neural Repair, 22(5), 505-513. http://doi.org/10.1177/1545968308317531

Schuster-Amft, C., Eng, K., Lehmann, I., Schmid, L., Kobashi, N., Thaler, I., ... Kiper, D. (2014). Using mixed methods to evaluate efficacy and user expectations of a virtual reality-based training system for upper-limb recovery in patients after stroke: a study protocol for a randomised controlled trial. Trials, 15, 350. http://doi.org/10.1186/17456215-15-350

Sears, E. D., \& Chung, K. C. (2010). Validity and responsiveness of the Jebsen-Taylor Hand Function Test. The Journal of Hand Surgery, 35(1), 30-37. http://doi.org/10.1016/j.jhsa.2009.09.008

Seo, N. J., Fischer, H. W., Bogey, R. A., Rymer, W. Z., \& Kamper, D. G. (2011). Use of visual force feedback to improve digit force direction during pinch grip in persons with stroke: a 
pilot study. Archives of Physical Medicine and Rehabilitation, 92(1), 24-30.

http://doi.org/10.1016/j.apmr.2010.08.016

Shaw, L., Rodgers, H., Price, C., van Wijck, F., Shackley, P., Steen, N., .. BoTULS investigators. (2010). BoTULS: a multicentre randomised controlled trial to evaluate the clinical effectiveness and cost-effectiveness of treating upper limb spasticity due to stroke with botulinum toxin type A. Health Technology Assessment (Winchester, England), 14(26), 1-113, iii-iv. http://doi.org/10.3310/hta14260

Shaw, S. E., Morris, D. M., Uswatte, G., McKay, S., Meythaler, J. M., \& Taub, E. (2005). Constraint-induced movement therapy for recovery of upper-limb function following traumatic brain injury. Journal of Rehabilitation Research and Development, 42(6), 769778.

Shelton, F. D., Volpe, B. T., \& Reding, M. (2001). Motor impairment as a predictor of functional recovery and guide to rehabilitation treatment after stroke. Neurorehabilitation and Neural Repair, 15(3), 229-237.

Shumway-Cook, A., \& Woollacott, M. (2011). Motor Control: Translating Research into Clinical Practice (Fourth, North American Edition). Philadelphia: LWW.

Siebers, A., Oberg, U., \& Skargren, E. (2010). The effect of modified constraint-induced movement therapy on spasticity and motor function of the affected arm in patients with chronic stroke. Physiotherapy Canada. Physiothérapie Canada, 62(4), 388-396. http://doi.org/10.3138/physio.62.4.388

Simondson, J. A., Goldie, P., \& Greenwood, K. M. (2003). The Mobility Scale for Acute Stroke Patients: concurrent validity. Clinical Rehabilitation, 17(5), 558-564. http://doi.org/10.1191/0269215503cr650oa 
Simone, A., Rota, V., Tesio, L., \& Perucca, L. (2011). Generic ABILHAND questionnaire can measure manual ability across a variety of motor impairments. International Journal of Rehabilitation Research. Internationale Zeitschrift Für Rehabilitationsforschung. Revue Internationale De Recherches De Réadaptation, 34(2), 131-140. http://doi.org/10.1097/MRR.0b013e328343d4d3

Skirven, T., Osterman, L., Fedorczyk, J., \& Amadio, P. (2011). Rehabilitation of the hand and upper extremity. Philadelphia, PA: Elsevier Mosby. Retrieved from http://www.mdconsult.com/public/book/view?title=Skirven:+Rehabilitation+of+the+Han d+and +Upper+Extremity

Slobogean, G. P., Noonan, V. K., Famuyide, A., \& O’Brien, P. J. (2011). Does objective shoulder impairment explain patient-reported functional outcome? A study of proximal humerus fractures. Journal of Shoulder and Elbow Surgery / American Shoulder and Elbow Surgeons, 20(2), 267-272. http://doi.org/10.1016/j.jse.2010.06.005

Sollerman, C., \& Ejeskär, A. (1995). Sollerman hand function test. A standardised method and its use in tetraplegic patients. Scandinavian Journal of Plastic and Reconstructive Surgery and Hand Surgery / Nordisk Plastikkirurgisk Forening [and] Nordisk Klubb for Handkirurgi, 29(2), 167-176.

Soyuer, F., \& Soyuer, A. (2005). Ischemic stroke: Motor impairment and disability with relation to age and lesion location. Journal of Neurological Sciences (Turkish), 22(1), 043-049.

Stamm, T. A., Cieza, A., Machold, K. P., Smolen, J. S., \& Stucki, G. (2004). Content comparison of occupation-based instruments in adult rheumatology and musculoskeletal rehabilitation based on the International Classification of Functioning, Disability and Health. Arthritis and Rheumatism, 51(6), 917-924. http://doi.org/10.1002/art.20842 
Stern, E. B. (1992). Stability of the Jebsen-Taylor Hand Function Test across three test sessions. The American Journal of Occupational Therapy: Official Publication of the American Occupational Therapy Association, 46(7), 647-649.

Sullivan, J. E., Hurley, D., \& Hedman, L. D. (2012). Afferent stimulation provided by glove electrode during task-specific arm exercise following stroke. Clinical Rehabilitation, 26(11), 1010-1020. http://doi.org/10.1177/0269215512442915

Sunderland, A., Tinson, D., Bradley, L., \& Hewer, R. L. (1989). Arm function after stroke. An evaluation of grip strength as a measure of recovery and a prognostic indicator. Journal of Neurology, Neurosurgery, and Psychiatry, 52(11), 1267-1272.

Suzuki, M., Yamada, S., Omori, M., Hatakeyama, M., Sugimura, Y., Matsushita, K., \& Tagawa, Y. (2008). Development of the upper-body dressing scale for a buttoned shirt: a preliminary correlational study. American Journal of Physical Medicine \& Rehabilitation / Association of Academic Physiatrists, 87(9), 740-749. http://doi.org/10.1097/PHM.0b013e31818378b0

Taub, E., Uswatte, G., Bowman, M., Mark, V., Delgado, A., Bryson, C., ... Bishop-McKay, S. (2013). Constraint-induced movement therapy combined with conventional neurorehabilitation techniques in chronic stroke patients with plegic hands: a case series. Archives of Physical Medicine and Rehabilitation, 94(1), 86-94. http://doi.org/10.1016/j.apmr.2012.07.029

Timmermans, A. A. A., Lemmens, R. J. M., Monfrance, M., Geers, R. P. J., Bakx, W., Smeets, R. J. E. M., \& Seelen, H. A. M. (2014). Effects of task-oriented robot training on arm function, activity, and quality of life in chronic stroke patients: a randomized controlled 
trial. Journal of Neuroengineering and Rehabilitation, 11, 45.

http://doi.org/10.1186/1743-0003-11-45

Turk, R., Burridge, J. H., Davis, R., Cosendai, G., Sparrow, O., Roberts, H. C., ... Schulman, J. (2008). Therapeutic effectiveness of electric stimulation of the upper-limb poststroke using implanted microstimulators. Archives of Physical Medicine and Rehabilitation, 89(10), 1913-1922. http://doi.org/10.1016/j.apmr.2008.01.030

Tyser, A. R., Beckmann, J., Franklin, J. D., Cheng, C., Hon, S. D., Wang, A., \& Hung, M. (2014). Evaluation of the PROMIS physical function computer adaptive test in the upper extremity. The Journal of Hand Surgery, 39(10), 2047-2051.e4. http://doi.org/10.1016/j.jhsa.2014.06.130

Tyson, S. F., \& DeSouza, L. H. (2004). Reliability and validity of functional balance tests post stroke. Clinical Rehabilitation, 18(8), 916-923.

Tyson, \& Turner. (1999). The process of stroke rehabilitation: what happens and why. Clinical Rehabilitation, 13(4), 322-332.

Tyson, \& Turner. (2000). Discharge and follow-up for people with stroke: what happens and why. Clinical Rehabilitation, 14(4), 381-392.

Urbin, M. A., Waddell, K. J., \& Lang, C. E. (2015). Acceleration metrics are responsive to change in upper extremity function of stroke survivors. Archives of Physical Medicine and Rehabilitation, 96(5), 854-861. http://doi.org/10.1016/j.apmr.2014.11.018

U.S. Department of Health and Human Services FDA Center for Drug Evaluation and Research, U.S. Department of Health and Human Services FDA Center for Biologics Evaluation and Research, \& U.S. Department of Health and Human Services FDA Center for Devices and Radiological Health. (2006). Guidance for industry: patient-reported 
outcome measures: use in medical product development to support labeling claims: draft guidance. Health and Quality of Life Outcomes, 4, 79. http://doi.org/10.1186/1477-75254-79

Uswatte, G., Taub, E., Morris, D., Light, K., \& Thompson, P. A. (2006). The Motor Activity Log-28: assessing daily use of the hemiparetic arm after stroke. Neurology, 67(7), 11891194. http://doi.org/10.1212/01.wnl.0000238164.90657.c2

Uswatte, G., Taub, E., Morris, D., Vignolo, M., \& McCulloch, K. (2005). Reliability and validity of the upper-extremity Motor Activity Log-14 for measuring real-world arm use. Stroke; a Journal of Cerebral Circulation, 36(11), 2493-2496.

van Delden, A. L. E. Q., Peper, C. L. E., Beek, P. J., \& Kwakkel, G. (2013). Match and mismatch between objective and subjective improvements in upper limb function after stroke. Disability and Rehabilitation, 35(23), 1961-1967. http://doi.org/10.3109/09638288.2013.768303

van Delden, A. L. E. Q., Peper, C. L. E., Nienhuys, K. N., Zijp, N. I., Beek, P. J., \& Kwakkel, G. (2013). Unilateral versus bilateral upper limb training after stroke: the Upper Limb Training After Stroke clinical trial. Stroke; a Journal of Cerebral Circulation, 44(9), 2613-2616. http://doi.org/10.1161/STROKEAHA.113.001969

van der Lee, J. H., Beckerman, H., Knol, D. L., de Vet, H. C. W., \& Bouter, L. M. (2004). Clinimetric properties of the motor activity log for the assessment of arm use in hemiparetic patients. Stroke; a Journal of Cerebral Circulation, 35(6), 1410-1414. http://doi.org/10.1161/01.STR.0000126900.24964.7e 
van der Lee, J. H., Beckerman, H., Lankhorst, G. J., \& Bouter, L. M. (2001). The responsiveness of the Action Research Arm test and the Fugl-Meyer Assessment scale in chronic stroke patients. Journal of Rehabilitation Medicine, 33(3), 110-113.

van der Lee, J. H., Wagenaar, R. C., Lankhorst, G. J., Vogelaar, T. W., Devillé, W. L., \& Bouter, L. M. (1999). Forced use of the upper extremity in chronic stroke patients: results from a single-blind randomized clinical trial. Stroke; a Journal of Cerebral Circulation, 30(11), $2369-2375$.

van de Ven-Stevens, L. A. W., Graff, M. J. L., Selles, R. W., Schreuders, T. A. R., van der Linde, H., Spauwen, P. H., \& Geurts, A. C. H. (2016). Instruments for assessment of impairments and activity limitations in patients with hand conditions: A European Delphi study. Journal of Rehabilitation Medicine, 47(10), 948-956. http://doi.org/10.2340/16501977-2015

Veehof, M. M., Sleegers, E. J. A., van Veldhoven, N. H. M. J., Schuurman, A. H., \& van Meeteren, N. L. U. (2002). Psychometric qualities of the Dutch language version of the Disabilities of the Arm, Shoulder, and Hand questionnaire (DASH-DLV). Journal of Hand Therapy: Official Journal of the American Society of Hand Therapists, 15(4), 347354.

Velozo, C. A., Seel, R. T., Magasi, S., Heinemann, A. W., \& Romero, S. (2012). Improving measurement methods in rehabilitation: core concepts and recommendations for scale development. Archives of Physical Medicine and Rehabilitation, 93(8 Suppl), S154-163.

Velstra, I. M., Ballert, C. S., \& Cieza, A. (2011). A systematic literature review of outcome measures for upper extremity function using the international classification of 
functioning, disability, and health as reference. $P M \& R$ : The Journal of Injury, Function, and Rehabilitation, 3(9), 846-860. http://doi.org/10.1016/j.pmrj.2011.03.014

Venkataraman, S. T., \& Iberall, T. (2012). Dextrous Robot Hands. Springer Science \& Business Media.

Vos-Vromans, D. C. W. M., de Bie, R. A., Erdmann, P. G., \& van Meeteren, N. L. U. (2005). The responsiveness of the ten-meter walking test and other measures in patients with hemiparesis in the acute phase. Physiotherapy Theory and Practice, 21(3), 173-180.

Vuillermot, S., Pescatore, A., Holper, L., Kiper, D. C., \& Eng, K. (2009). An extended drawing test for the assessment of arm and hand function with a performance invariant for healthy subjects. Journal of Neuroscience Methods, 177(2), 452-460. http://doi.org/10.1016/j.jneumeth.2008.10.018

Wagner, J. M., Rhodes, J. A., \& Patten, C. (2008). Reproducibility and minimal detectable change of three-dimensional kinematic analysis of reaching tasks in people with hemiparesis after stroke. Physical Therapy, 88(5), 652-663. http://doi.org/10.2522/ptj.20070255

Wang, T., Lin, K., Wu, C., Chung, C., Pei, Y., \& Teng, Y. (2011). Validity, responsiveness, and clinically important difference of the ABILHAND questionnaire in patients with stroke. Archives of Physical Medicine and Rehabilitation, 92(7), 1086-1091.

Washington University in St. Louis. (2009). Men in Nursing, Occupational Therapy, \& Physical Therapy. Bernard Becker Medical Library. Retrieved from http://beckerexhibits.wustl.edu/mowihsp/stats/men.htm

Weng, L. Y., Hsieh, C. L., Tung, K. Y., Wang, T. J., Ou, Y. C., Chen, L. R., .. Liu, C. F. (2010). Excellent reliability of the Sollerman hand function test for patients with burned 
hands. Journal of Burn Care \& Research: Official Publication of the American Burn Association, 31(6), 904-910. http://doi.org/10.1097/BCR.0b013e3181f93583

WHO. (2001). International Classification of Functioning, Disability and Health. Geneva: World Health Organization.

Williams, G., Robertson, V., Greenwood, K., Goldie, P., \& Morris, M. E. (2006). The concurrent validity and responsiveness of the high-level mobility assessment tool for measuring the mobility limitations of people with traumatic brain injury. Archives of Physical Medicine and Rehabilitation, 87(3), 437-442. http://doi.org/10.1016/j.apmr.2005.10.028

Wilson, D., Baker, L., \& Craddock, J. (1984). Functional Test for the Hemiparetic Upper Extremity. American Journal of Occupational Therapy, 38(3), 159-164.

Winstein, C. J., Wolf, S. L., Dromerick, A. W., Lane, C. J., Nelsen, M. A., Lewthwaite, R., ... ICARE Investigative Team. (2013). Interdisciplinary Comprehensive Arm Rehabilitation Evaluation (ICARE): a randomized controlled trial protocol. BMC Neurology, 13, 5. http://doi.org/10.1186/1471-2377-13-5

Wolf, S. L., Catlin, P. A., Ellis, M., Archer, A. L., Morgan, B., \& Piacentino, A. (2001). Assessing Wolf motor function test as outcome measure for research in patients after stroke. Stroke; a Journal of Cerebral Circulation, 32(7), 1635-1639.

Wolf, S. L., Winstein, C. J., Miller, J. P., Taub, E., Uswatte, G., Morris, D., ... EXCITE Investigators. (2006). Effect of constraint-induced movement therapy on upper extremity function 3 to 9 months after stroke: the EXCITE randomized clinical trial. JAMA, 296(17), 2095-2104. http://doi.org/10.1001/jama.296.17.2095

Woodbury, M. L., Velozo, C. A., Richards, L. G., Duncan, P. W., Studenski, S., \& Lai, S. M. (2008). Longitudinal stability of the Fugl-Meyer Assessment of the upper extremity. 
Archives of Physical Medicine and Rehabilitation, 89(8), 1563-1569.

http://doi.org/10.1016/j.apmr.2007.12.041

Wood-Dauphinee, S. L., Williams, J. I., \& Shapiro, S. H. (1990). Examining outcome measures in a clinical study of stroke. Stroke; a Journal of Cerebral Circulation, 21(5), 731-739.

Wu, C. W., Seo, H. J., \& Cohen, L. G. (2006). Influence of electric somatosensory stimulation on paretic-hand function in chronic stroke. Archives of Physical Medicine and Rehabilitation, 87(3), 351-357. http://doi.org/10.1016/j.apmr.2005.11.019

Yozbatiran, N., Der-Yeghiaian, L., \& Cramer, S. C. (2008). A standardized approach to performing the Action Research Arm Test. Neurorehabilitation and Neural Repair, 22(1), 78-90. http://doi.org/10.1177/1545968307305353 